Flora Di Donato, Élodie Garros, Anne Lavanchy, Pascal Mahon, Tania Zittoun

\title{
LA FABRIQUE DE L'INTÉGRATION
}

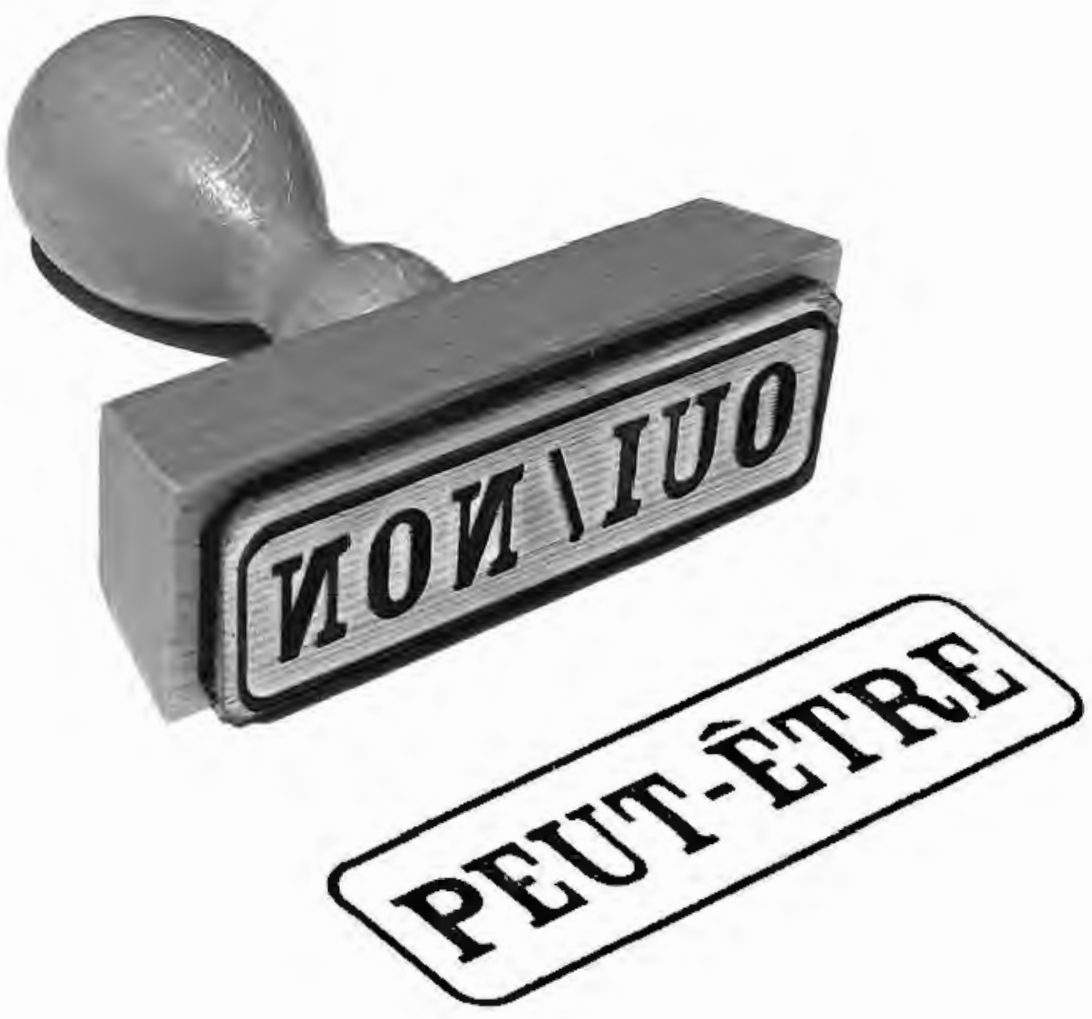



LA FABRIQUE DE L'INTÉGRATION 


\section{REMERCIEMENTS}

L'édition de ce livre a reçu le soutien de la Commission des publications de l'Université de Neuchâtel et de la Haute École de travail social HESSO Genève.

L'étape de la prépresse de cette publication a été soutenue par le Fonds national suisse de la recherche scientifique.
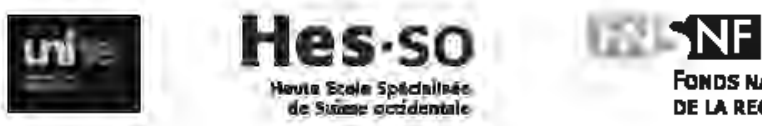

FONDS NATIONAL SUISSE DE LA RECHERCHE SOEMTIFIQUE

Les Éditions Antipodes bénéficient d'une prime d'encouragement de l'Office fédéral de la culture pour les années 2019-2020.

\section{MISE EN PAGE}

Fanny Tinner | chezfanny.ch

\section{CORRECTION}

Adeline Vanoverbeke

\section{COUVERTURE}

(C) Plonk \& Replonk

\section{(c) (1) (8) (9)}

Ce texte est sous licence Creative Commons: elle vous oblige, si vous utilisez cet écrit, à en citer l'auteur.e, la source et l'éditeur original, sans modification du texte ou de l'extrait et sans utilisation commerciale.

(C) 2020, Éditions Antipodes

École-de-Commerce 3, 1004 Lausanne, Suisse www.antipodes.ch - editions@antipodes.ch

DOI : $10.33056 /$ ANTIPODES.11704

Papier, ISBN : 978-2-88901-170-4

PDF, ISBN : 978-2-88901-974-8

EPUB, ISBN : 978-2-88901-975-5 
Flora Di Donato, Élodie Garros, Anne Lavanchy,

Pascal Mahon et Tania Zittoun

\section{LA FABRIQUE DE L'INTÉGRATION}

Antip.odes Collection Regards anthropologiques 

À Armand, Bruno, Charles, Dominique, Elvire, François, Gilbert, Johan, Kirin et Line, ainsi qu’à toutes les personnes qui vivent ou ont vécu un parcours semblable

À Corentin, Corinne, Costin, Jules, Juliette, Justine, Michèle, Nadège, Nasser, Natacha, Nathan, Noël, Norma et Norman, ainsi qu'à toutes les personnes qui ont pour mission d'appliquer la loi 



\section{AVANT-PROPOS}

a question de l'intégration, qui est au cœur de notre législation en matière de migration, en particulier de la procédure de naturalisation mise en place par la loi sur la nationalité suisse du 29 septembre 1952, entièrement révisée en 2014, a toujours fait débat. Elle est même parvenue, quelquefois, à être sous les feux de la rampe, tantôt à travers un événement culturel, comme l'a été, en 1978, la sortie du film Les Faiseurs de Suisses du cinéaste zurichois Rolf Lissy, qui a connu un très large succès public ${ }^{1}$, tantôt à travers l'actualité judiciaire, comme dans le cas du fameux arrêt Emmen du Tribunal fédéral, du 9 juillet 2003. Par ce jugement, qui a provoqué une tempête médiatique et politique, la plus haute Cour du pays a annulé d'un trait de plume une bonne partie des décisions de refus d'octroi de la naturalisation prononcées en votation, le 12 mars 2000, par le peuple de la commune lucernoise du même nom, à l'encontre de 48 candidates et candidats en provenance principalement des Balkans ${ }^{2}$. La chronique médiatique a elle aussi régulièrement alimenté le débat, notamment avec le cas d'Irving Dunn, ancien professeur à l'École polytechnique de Zurich, en Suisse depuis plus de quarante ans, à qui l'assemblée du district d'Einsiedeln, dans le canton de Schwyz, a refusé la naturalisation en octobre 2014 au motif qu'il n'était pas assez intégré ${ }^{3}$, ou celui

1. Sorti en Suisse alémanique d'abord, avec Emil Steinberger et Walo Lüönd dans les rôles principaux, Die Schweizermacher a été l'un des plus grands succès du cinéma suisse, avec plus de 940000 entrées en Suisse, où il est resté au sommet du box-office jusqu'en 1997, avant d'être détrôné par Titanic.

2. ATF 129 I 217, A. und Mitb. gegen Einwohnergemeinde Emmen und Regierungsrat des Kantons Luzern, du 9 juillet 2003.

3. Voir, entre autres, sur ce cas et pour la réaction d'incompréhension et d'irritation de l'intéressé, lequel a finalement renoncé, en janvier 2015, à déposer une nouvelle demande, un article de 20 minutes, du 15 octobre 2014, http://www.20min.ch/ro/news/suisse/story/ En-Suisse-depuis-43-ans--il-ne-sera-pas-naturalise-31355807, et un autre de la Luzerner Zeitung, du 15 janvier 2015, http://www.luzernerzeitung.ch/nachrichten/zentralschweiz/ schwyz/Professor-verzichtet-auf-Einbuergerungsgesuch; art96,470338. 
de la jeune Funda Yilmaz, également recalée dans un premier temps par la commission des naturalisations de la commune de Buchs, en Argovie, après une audition et une série de questions des plus "ubuesques" ${ }^{4}$. Ou encore, plus récemment, le cas de la famille Scanio, de Nyon, qui a lui aussi déclenché une vague de réactions dans les médias. D'après ceux-ci, cette famille aurait été particulièrement mal traitée lors de son audition par la commission communale compétente, en date du 24 octobre 2017, commission qui, à la suite de l'audition, avait rendu un préavis négatif pour les deux époux, pour motif d'«insuffisance de connaissances en civisme» ${ }^{5}$. S'estimant malmenés et humiliés, les époux s'étaient alors plaints dans les médias du "climat "délétère" de l'audition et [du] comportement de certains commissaires» et la commune avait finalement octroyé la nationalité suisse au père, Salvatore, d'origine italienne, ainsi qu'aux deux enfants, tout en la refusant à la mère, Antonia, d'origine portugaise ${ }^{6}$.

À la lumière de ces quelques cas récents ${ }^{7}$, force est de constater que l'expression "les faiseurs de Suisses", mise en avant il y a quarante ans par le film évoqué, et que l'on croyait devoir être rangée au rang des accessoires du $7^{\mathrm{e}}$ art et, plus encore, du siècle passé, est en réalité toujours actuelle et pertinente. La situation, en dépit des années écoulées depuis lors et malgré plusieurs modifications de la législation, n'aurait-elle pas réellement changé? Qui plus est, derrière ces "pics» d'actualité, souvent bien loin de

4. Sur ce cas, relaté même par la presse internationale, et les 92 questions «ubuesques» posées, voir un article du journal Le Matin et un autre du journal Le Temps, https://www. lematin.ch/suisse/trompe-sport-national-passeport/story/11488187 et https://www.letemps. $\mathrm{ch} /$ suisse/funda-yilmaz-largovienne-plier-faiseurs-suisse. Cette jeune candidate, née "en Suisse de parents turcs installés là depuis quarante ans", qui "maitrise l'allemand et le dialecte, travaille comme dessinatrice en génie civil à Aarau, n’a pas de dettes ni de casier judiciaire et [...] s'est éprise d'un Suisse, qu'elle a l'intention d'épouser l'an prochain ", aurait été recalée parce qu'elle avait répondu, à la question d'un sport typiquement helvétique, le ski et non le hornuss ou la lutte à la culotte (!), et ce malgré un sans-faute à l'examen écrit.

5. Voir notamment, sur ce cas, pour une description du déroulement de l'audition, l'article du journal Le Temps, du 8 décembre 2017, https://www.letemps.ch/suisse/ faiseurs-suisses-sevissent-nyon.

6. Voir l'article du journal Le Temps, du 31 janvier 2018, https://www.letemps.ch/suisse/ secondo-recale-volteface-nyon, ainsi qu'un article de 24 heures, du 17 avril 2018, https:// www.24heures.ch/vaud-regions/la-cote/nyon-coupe-poire-deux-affaire-faiseurs-suisse/ story/30510064.

7. Pour d'autres cas, dont un tout récent, qui montre que si les lois changent, les pratiques perdurent, voir notamment https://www.rts.ch/info/regions/autres-cantons/11048497une-naturalisation-ne-peut-pas-etre-refusee-pour-des-lacunes-mineures.html (relatif à un arrêt du Tribunal fédéral du 18 décembre 2019, TF 1D_1/2019); plus généralement, cf. aussi l'étude de la Commission fédérale contre le racisme, Discrimination dans le cadre des naturalisations, Avis de la CFR sur la situation actuelle, Berne, septembre 2017, http://www. ekr.admin.ch/pdf/Einbuergerung_F_version_web692e.pdf. 
la scène médiatique, la procédure de naturalisation et la question de l'intégration, qui permettent de "fabriquer" des Suissesses et des Suisses, ou de refuser ce «label» à des candidates et candidates qui y aspirent, touchent chaque année des milliers de personnes, anonymes, beaucoup plus anonymes que les protagonistes des Faiseurs de Suisses ou des cas d'Emmen, d'Einsiedeln ou de Nyon ${ }^{8}$.

La Suisse est, selon plusieurs recherches, l'un des pays les plus restrictifs du continent en matière d'octroi de la nationalité: «En général, les résultats montrent que la législation suisse compte parmi les plus restrictives d'Europe en ce qui concerne l'acquisition de la nationalité du fait de la naissance sur le territoire (droit du sol à la naissance) et de la résidence à long terme (naturalisation ordinaire).»" Une telle politique restrictive a des conséquences humaines et sociales. Ces refus d'accorder la nationalité, même s'ils ne sont parfois que provisoires, comme dans les cas de Buchs et de Nyon, ont évidemment des conséquences psychologiques, provoquant frustration, sentiment d'injustice, voire sentiment de rejet ou d'exclusion. Alors que jusque-là les personnes concernées se considéraient comme des candidates légitimes, des personnes menant une vie "normale», des personnes «intégrées», une telle procédure a des incidences importantes sur leur parcours de vie. Ces refus questionnent parfois aussi la légitimité même des autorités et peuvent aller jusqu'à remettre en cause le principe des commissions de naturalisation qui jugent les candidates et candidats à la nationalité ${ }^{10}$.

8. C’est le lieu de signaler que le canton de Neuchâtel a décidé, en 2010, pour des raisons de protection des données personnelles, de ne plus publier dans la Feuille officielle les noms des personnes qui ont obtenu la naturalisation dans le canton, mais une simple statistique des cas d'octroi du droit de cité cantonal (voir https://www.ne.ch/medias/archives/CP_NEAT/2010/ commCE16juin2010VF.pdf, pour le communiqué de presse du Conseil d'État du 16 juin 2010, ainsi que, pour la statistique, https://www.ne.ch/legislation-jurisprudence/pubfo/ ArrRegCE/Pages/statistique-naturalisations.aspx).

9. Voir, à ce propos, l'indicateur publié sur le site du nccr - on the move, https:// indicators.nccr-onthemove.ch/comment-les-pays-deurope-regulent-ils-lacces-a-lacitoyennete/?lang=fr, ainsi que, pour des comparaisons internes à la Suisse, http://nccronthemove.ch/DataManagement/Visualization/Embed/Naturalization_Rates.html, et http:// nccr-onthemove.ch/DataManagement/Visualization/Embed/LegalIndicators.html; voir aussi Dragan Ilic, Naturalisations et préjugés: ce qu’on sait - ce qui n'est pas clair, 5 janvier 2017, nccr - on the move, http://nccr-onthemove.ch/wp_live14/wp-content/uploads/2017/02/ Policy-Brief-nccr-on-the-move-05-Dragan-Ilic-FR-Web.pdf.

10. C'est ainsi, par exemple, que la Ville de Genève a, par décision du Conseil municipal du 16 janvier 2018, opté pour la suppression de sa Commission des naturalisations; voir l'article de la Tribune de Genève, du 17 janvier 2018, https://www.tdg.ch/geneve/ actu-genevoise/ville-renonce-faiseurs-suisses/story/26258462. 
L'ambition du projet de recherche qui est à la base du présent ouvrage était précisément de chercher à comprendre ce qui se passe concrètement dans des procédures de naturalisation et à cerner cette notion d'intégration qui est au cour de la législation sur la naturalisation, c'est-à-dire l'octroi de la nationalité suisse ${ }^{11}$, mais aussi, depuis un certain temps, de la législation sur le séjour et l'établissement des étrangers dans notre pays ${ }^{12}$. Nous avons voulu tenter de comprendre pourquoi et comment la nationalité est si parcimonieusement accordée et par quels mécanismes la loi - car c'est bien, dans le fond, de la loi qu'il s'agit - se trouvait appliquée dans des modalités invisibles dont les effets sont parfois surprenants.

Financé par le Fonds national suisse de la recherche scientifique (requête 147287) et mené à l'Université de Neuchâtel, de 2013 à 2017, par cinq chercheuses et chercheur en droit et en sciences sociales (les auteures ${ }^{13} \mathrm{du}$ présent ouvrage), ce projet interdisciplinaire avait dans un premier temps été conçu avec un double objectif et une double ambition. D'un côté, il avait pour but d'analyser et de "reconstruire» la notion d'intégration telle qu'elle était apparue dans le corpus juridique au cours du $\mathrm{XX}^{\mathrm{e}}$ siècle, et telle qu'elle avait été, était et est mise en œuvre, dans l'actualité, par certaines instances administratives et judiciaires. L'idée était donc de retracer la genèse et l'évolution de la notion d'intégration telle qu'elle s'était forgée dans l'esprit et dans l'œuvre du législateur, puis à travers la mise en ouvre de la loi par l'administration et le juge. De l'autre côté, le projet entendait aussi examiner et mettre en lumière l'impact de cette notion dans les trajectoires et parcours de vie des personnes candidates à la naturalisation. Nous avons donc, dans la partie empirique de la recherche, investigué et reconstruit les trajectoires personnelles et les trajectoires administrativo-légales d'un certain nombre de personnes qui ont fait la démarche concrète de demande de la naturalisation dans le canton de Neuchâtel. Nos analyses se sont fondées à cet égard sur l'examen des dossiers personnel et administratif (auquel les administrations

11. La loi sur l'acquisition et la perte de la nationalité suisse (loi sur la nationalité, LN), du 29 septembre 1952, remplacée, à partir du $1^{\text {er }}$ janvier 2018, par la nouvelle loi fédérale sur la nationalité (LN), du 20 juin 2014, fruit d'une révision totale de la précédente.

12. Législation qui, à partir du $1^{\text {er }}$ juillet 2018, a même pris le titre, nouveau, de loi sur les étrangers et l'intégration (LEE), au lieu de loi sur le séjour et l'établissement des étrangers (LSEE), dans un premier temps, de 1931 au 31 décembre 2007, puis de loi sur les étrangers (LEtr), dans un deuxième temps, du $1^{\text {er }}$ janvier 2008 au 30 juin 2018.

13. Nous utiliserons dans tout l'ouvrage, par souci de simplification, le «nous» féminin pluriel, qui inclut aussi le masculin. 
intéressées nous ont donné accès, avec l'accord bien évidemment des personnes concernées) et sur des entretiens approfondis avec ces personnes. La recherche avait ainsi pour ambition non seulement de refléter la perspective des autorités, mais aussi de donner voix aux personnes engagées dans des procédures administratives et légales de naturalisation, en retraçant la complexité et les effets du processus d'intégration de leur point de vue et sur leurs trajectoires et parcours de vie. Au cours de nos travaux, un troisième axe de recherche a cependant rapidement émergé et s'est imposé comme un passage - un pont - obligé entre les deux premiers: la perspective - et l'œuvre créatrice - des personnes employées dans les administrations cantonales et communales qui appliquent, concrètement et quotidiennement, la législation en question, qui la travaillent et qui la façonnent au gré des cas concrets dont elles ont à s'occuper et des procédures qu' elles ont à mener, et qui sont aussi, à leur tour, et dans une moindre mesure, façonnées par celles-ci.

Nous avons donc voulu - et cherché à - comprendre comment l'intégration posée et imposée par le haut ou en amont, en quelque sorte, par le législateur, est perçue et vécue, en aval, par les personnes à qui elle s'impose, c'est-à-dire par les personnes candidates à la naturalisation (et, dans le domaine du droit des étrangers, aux personnes migrantes qui souhaitent obtenir un droit de séjour en Suisse), d'une part. Mais aussi, d'autre part, aux personnes qui sont chargées de mettre en œuvre cette exigence d'intégration dans la réalité concrète, c'est-à-dire les agentes et les agents des administrations dont c'est la mission.

Inscrit dans une approche interdisciplinaire, notre projet avait des objectifs et des ambitions relativement larges. L'approche interdisciplinaire choisie a entraîné tout d'abord un certain nombre de contraintes et de difficultés, notamment d'un point de vue méthodologique, sur lesquelles nous reviendrons largement par la suite (chapitres 1 et 2).

À ce stade, il convient toutefois de mettre en exergue une autre contrainte, ou difficulté, liée à la fois aux ambitions du projet et au choix ou à la sélection des cas concrets. S'agissant de ses ambitions, le projet visait, on l'a dit, à cerner et à chercher à comprendre la notion d'intégration qui est au cœur de la législation migratoire, c'est-à-dire aussi bien la législation sur la naturalisation et la nationalité que celle sur le séjour et l'établissement des étrangers. En effet, si le thème de l'intégration est devenu central dans la 
politique publique suisse à partir des années 1990, c'est à travers diverses révisions de deux actes législatifs fondamentaux en matière de politique migratoire qu'il s'est progressivement imposé. D'une part, la loi fédérale sur l'acquisition et la perte de la nationalité suisse, du 29 septembre 1952 (ancienne loi sur la nationalité, LN), révisée d'abord partiellement, en 1990, ainsi qu'en 2003, 2004 et 2007, puis objet d'une révision totale en juin 2014 (nouvelle loi sur la nationalité, $\mathrm{nLN})^{14}$. D'autre part, la loi fédérale sur les étrangers, du 16 décembre 2005 (loi sur les étrangers, LEtr, ou maintenant loi sur les étrangers et l'intégration, LEI), elle-même fruit d'une révision totale de l'ancienne loi sur le séjour et l'établissement des étrangers (LSEE), du 26 mars 1931, et déjà plusieurs fois révisée partiellement. Un des objets de ces diverses révisions était justement d'introduire, puis de renforcer et, en dernier lieu, d'harmoniser la notion d'intégration dans ces différents textes législatifs.

Or, si la partie théorique de notre projet, dédiée à l'analyse juridique et à la "reconstruction" de la notion d'intégration telle qu'elle s'est développée dans le système législatif suisse, a pu prendre en compte aussi bien le volet du droit de la nationalité et de la naturalisation que celui du droit "ordinaire» des étrangers, il n'en est pas allé de même de la partie empirique de la recherche. Celle-ci a en effet dû se limiter à un nombre restreint de cas - ne serait-ce qu'en raison du temps, important, lié au traitement de ces cas, mais aussi pour des motifs de disponibilité des cas et, surtout, des personnes concernées. Au total, une dizaine de cas, dont cinq ont pu faire l'objet d'une analyse complète, à savoir d'une étude des dossiers à la fois personnel et administratif, d'un ou plusieurs entretiens approfondis avec la ou les personnes concernées, c'est-àdire les candidates et candidats eux-mêmes, ainsi que d'une analyse des trajectoires de vie et du parcours administrativo-légal.

Il s'est avéré en outre que les cas concrets auxquels nous avons pu avoir accès, et pour lesquels nous avons obtenu l'accord des personnes concernées, se situaient pratiquement tous dans le domaine du droit de la naturalisation - et non dans celui du droit (de séjour) des étrangers. Il en résulte ainsi, dans la partie empirique du projet, un accent qui penche clairement du côté de l'un des deux volets du système normatif abritant et utilisant la notion d'intégration. Nous espérons néanmoins pouvoir montrer

14. La nouvelle loi porte l'abréviation officielle LN ; toutefois, nous l'abrégerons ici nLN, pour la distinguer de l'ancienne loi. 
que l'étude empirique de ces cas et les analyses scientifiques qui ont pu en être tirées ont et gardent une valeur générale.

Il résulte également de la sélection opérée dans les cas concrets examinés dans la partie empirique de notre recherche que celle-ci porte sur un nombre restreint de cas, lesquels ne sauraient dès lors être considérés comme statistiquement ou numériquement suffisants pour constituer un échantillon représentatif de l'ensemble des procédures de naturalisation qui se déroulent en Suisse. La manière même dont ces cas ont été choisis, à l'aide et au travers de signalements des administrations compétentes, ainsi qu'avec l'exigence du consentement des personnes directement intéressées, implique également un biais dans la représentativité de ces cas: on peut en effet supposer que ces cas sont, pour certains au moins, des cas "atypiques", voire des cas plutôt "problématiques», qui ne sont pas nécessairement représentatifs de la réalité du canton de Neuchâtel, plutôt connu, d'ailleurs, pour un taux important d'octroi de la nationalité.

Néanmoins, là encore, ces cas nous semblent permettre des analyses scientifiques qui gardent une valeur générale, sous l'angle des trajectoires de vie des personnes concernées, de leurs trajectoires administratives et légales ainsi que de leur engagement face à loi (leur agentivité légale), et l'analyse du travail des professionnelles et des professionnels de l'administration (matérialisation de la procédure dans un dossier et étude des processus de prise de décisions, cf. $3^{\text {e }}$ partie du présent ouvrage).

Une autre difficulté encore à laquelle notre projet a été confronté est celle de la "loi en mouvement» ou, plus précisément, des fréquents - pour ne pas dire perpétuels - changements de la législation pertinente, mais aussi, au-delà, de la loi, des nombreux changements de procédure et de nomenclature, notamment de la nomenclature servant à la désignation des services de l'administration, au gré des réorganisations et autres changements de gouvernements. Ainsi, pour prendre l'exemple le plus significatif, tous les cas concrets que nous avons étudiés dans la partie empirique de notre recherche se sont "déroulés» sous l'empire de ce qu'il conviendrait désormais d'appeler l'ancienne loi sur la nationalité, la loi sur l'acquisition et la perte de la nationalité suisse (loi sur la nationalité, LN), du 29 septembre 1952, laquelle est restée en vigueur jusqu'au 31 décembre 2017, mais ne l'est plus depuis le $1^{\text {er }}$ janvier 2018, puisqu'elle a été remplacée, à cette date, par la nouvelle loi sur la nationalité, du 20 juin 2014. 
Dès lors, au moment où ces lignes et le présent ouvrage seront lus, c'est cette nouvelle loi qui sera en vigueur et à laquelle il conviendra ou conviendrait de se référer. Nous nous référerons cependant ici, dans cet ouvrage, non pas, en principe, à cette nouvelle législation, mais bien à la législation en vigueur au moment où se sont déroulés les cas concrets examinés. Nous mentionnerons cependant, bien évidemment, là où cela s'avérera nécessaire, les éventuelles solutions différentes qu'apporte ou qu'apportera la nouvelle législation.

Comme pour les autres contraintes et difficultés évoquées plus haut - et même si la nouvelle loi sur la nationalité apporte certaines innovations de fond et/ou de procédure -, nous pensons que les enseignements tirés des analyses scientifiques proposées dans cet ouvrage mettent en évidence des mécanismes sociaux, administratifs et légaux complexes, qui perdurent au-delà des changements évoqués. Cette attention aux processus de fond signale donc la pertinence de notre recherche dans le contexte de la nouvelle réglementation, et de celles qui suivront.

Les différents cas concrets que nous avons étudiés dans la partie empirique de la recherche ont fait l'objet d'analyses scientifiques diverses, à l'aune de plusieurs perspectives et selon les différents ancrages disciplinaires des chercheuses et chercheur. Les résultats de ce travail de recherche sont exposés dans cet ouvrage, auquel nous avons choisi, à dessein, de donner le titre La fabrique de l'intégration, un titre qui peut peut-être paraître provocateur à certains égards. L'idée est de montrer que l'intégration, et en particulier dans le contexte de la naturalisation, n'est pas un état de fait scientifiquement exact, cautionné par la loi, mais constitue la somme de processus vivants, mis en œuvre, régulés, entravés, agis et vécus, subis ou soufferts, par de très nombreuses personnes, dans des postures et des rôles différents: candidates et candidats - ou "clientes et clients»-, employées et employés de l'administration, membres des autorités communales et cantonales, avocats, juges, etc.

Nous tenons finalement à exprimer notre gratitude la plus vive et sincère à toutes les personnes qui, à un titre ou à un autre, nous ont permis de réaliser cette recherche et de proposer le présent ouvrage. Il s'agit bien sûr des institutions qui, comme le Fonds national suisse de la recherche scientifique, l'Université de Neuchâtel (Facultés de droit et de lettres et sciences humaines) et la Haute École de Suisse occidentale - HETS Genève, ont soutenu ce projet, par le financement et l'infrastructure alloués. Il s'agit aussi 
et surtout des personnes, anonymes, qui l'ont rendu possible, grâce au temps et à la disponibilité, mais aussi à la confiance qu' elles nous ont accordés. Nous voulons parler ici, bien entendu, des personnes dont nous retraçons les cas, qui nous ont raconté leurs trajectoires et parcours de vie, nous ont ouvert leurs dossiers, et parfois leur porte, et qui ont partagé avec nous leurs espoirs et leurs désillusions, personnes que nous avons appelées - pour des raisons de protection des données - Armand, Bruno, Charles, Dominique, Elvire, François, Gilbert, Johan, Kirin et Line.

Nos remerciements vifs et sincères s'adressent aussi aux personnes qui, "de l'autre côté du guichet ", si l'on peut dire, c'est-à-dire au sein des services de l'État, le Service de la justice et le Service de la cohésion multiculturelle en particulier, mais aussi le Service des migrations et certaines Commissions des naturalisations et des agrégations, sont chargées de mettre en œuvre au quotidien la législation et d'appliquer cette notion d'intégration que nous cherchions à cerner. Ces personnes, que nous avons appelées - pour les mêmes raisons de protection des données - Corentin, Corinne, Costin, Jules, Juliette, Justine, Michèle, Nadège, Nasser, Natacha, Nathan, Noël, Norma et Norman, n'ont ménagé ni leur temps ni leurs efforts - et, souvent, leur patience - pour répondre à nos questions, discuter avec nous de nos hypothèses, réflexions et analyses. Elles l'ont fait notamment dans le cadre d'une journée, commune, de "restitution» des résultats de notre recherche, que certaines d'entre elles ont passée avec nous. Mais elles ont aussi accepté, tout au long de la recherche, de nous expliquer les pratiques de leur service et les rouages des institutions dans lesquelles elles exercent leur profession et assument leurs missions, notamment la manière dont elles exploitent - ou parfois craignent - la marge d'appréciation que le «droit» leur laisse.

Que toutes ces personnes, que nous ne pouvons nommer ici autrement que par les prénoms d'emprunt que nous leur avons attribués, soient chaleureusement remerciées.

Neuchâtel, janvier 2020 Pascal Mahon Flora Di Donato Élodie Garros Anne Lavanchy Tania Zittoun 



\section{PARTIE I}

INTRODUCTION 



\section{MODÉLISER LE PROJET: THÉORIE ET ÉPISTÉMOLOGIE}

TANIA ZITTOUN, FLORA DI DONATO, ÉLODIE GARROS, ANNE LAVANCHY ET PASCAL MAHON

\footnotetext{
C
} e livre explore la manière dont la Suisse a construit et utilise la notion d'intégration dans ses rapports à la population étrangère, en lien avec la procédure d'attribution de la nationalité suisse. Il en examine les aspects juridiques, la manière dont la loi est mise en œuvre par les personnes en charge de son application, et la façon dont des personnes étrangères font l'expérience des exigences d'intégration et des procédures de naturalisation. Il résulte d'un travail interdisciplinaire de recherche de plusieurs années, qui nous a amenées à comprendre que l'intégration et, en particulier, la naturalisation étaient moins un état de fait cautionné par la loi que de longs processus, mis en branle, régulés, entravés, agis et vécus par de très nombreuses personnes, dans des postures et des rôles différents. C'est cet aspect complexe et dynamique que nous avons voulu désigner par le titre, à dessein provocateur, de «fabrique» de l'intégration.

Ce chapitre introductif nous permet de poser le problème de base: qu'est-ce que «l'intégration"? (1.1). Nous présentons ensuite les différentes disciplines que nous avons convoquées (1.2), puis définissons plus spécifiquement la problématique que ce livre couvre (1.3). Un modèle nous permet d'analyser la fabrique de l'intégration à partir des points de vue des personnes impliquées (1.4), et nous conduira à proposer une série d'hypothèses exploratoires qui constituent les fils rouges de cet ouvrage, dont nous annoncerons rapidement la structure (1.5).

\subsection{DE L'INTÉGRATION}

Tout le monde a probablement son idée de ce qu' "être intégré» signifie. Mais dans un pays comme la Suisse, État fédéral composé de 26 cantons et de quatre langues nationales, de villes 
et de campagnes, de plaines et de montagnes, à quoi est-on intégré, qui définit l'intégration? Et qu'apporte l'intégration, à qui ? Lorsqu'elle est utilisée dans un contexte national, la notion d'intégration se réfere globalement au "processus par lequel des migrants deviennent acceptés dans une société donnée, en tant qu'individus et en tant que groupe ${ }^{1}$. Ce processus implique en l'occurrence des personnes n'ayant pas la nationalité du pays dans lequel elles sont établies, et qui engagent un certain nombre de démarches institutionnelles dans l'espoir que celles-ci débouchent sur une forme de reconnaissance qui leur permettra d'accéder à la nationalité du pays d'accueil, c'est-à-dire à un certain nombre de droits et de devoirs citoyens. Ainsi, en Suisse, où la question est traitée au niveau fédéral, au niveau cantonal et au niveau communal, une «intégration suffisante» est par exemple indiquée, comme nous le verrons, par le fait que la personne maîtrise la langue locale, qu'elle s'est toujours scrupuleusement acquittée du paiement de ses impôts ou, dans certains cas, que ses comportements ne sont pas trop «hors norme». Ces pratiques, traitées comme indices d'intégration ou de non-intégration, peuvent alors donner droit, ou non, à la naturalisation, c'est-à-dire aux démarches qui se concluent par l'acquisition de la nationalité suisse.

Le processus de naturalisation et la notion d'intégration, ou les notions qui l'ont précédée dans le corpus juridique (l'incorporation et l'assimilation), ont fait l'objet de débats et de discussions politiques souvent vifs depuis l'émergence, le développement et la consolidation de l'État-nation, à la fin du XIX ${ }^{\mathrm{e}}$ siècle. Ces notions ont depuis longtemps été travaillées par la philosophie, le droit et les sciences humaines et sociales. La problématique n'en demeure pas moins au centre des débats - parfois brûlants - aujourd'hui en Europe et en Suisse.

L'intégration est au cœur de notre investigation; nous proposons de l'aborder sous un angle nouveau, en retraçant les perspectives entrecroisées des différentes personnes qui participent au processus. Il est clair en effet que la notion d'intégration varie en fonction de ses utilisatrices et utilisateurs et de ses contextes d'utilisation. Les questions qui se posent alors sont: qui emploie cette notion, pour en faire quoi, avec quels résultats et dans quelles situations? Dans des démarches de naturalisation (mais aussi de prolongation de permis

1. Phenninx, 2003 [http://www.migrationpolicy.org/article/integration-role-communitiesinstitutions-and-state], consulté le 24 mars 2016. 
de résidence), on peut identifier quatre types d'actrices et d'acteurs principalement concernés: les actrices et les acteurs politico-législatifs, qui définissent la notion d'intégration et les critères permettant de l'évaluer; les actrices et les acteurs administratifs et judiciaires, dont la fonction est d'appliquer - et donc d'interpréter - ces principes politiques et législatifs auprès de la population, ou d'en contrôler l'application; les personnes candidates à la naturalisation elles-mêmes, qui sont tenues de mettre en scène des éléments susceptibles de démontrer leur intégration; et, finalement, les personnes tierces, qui côtoient plus ou moins directement les candidates et les candidats et prennent une part active dans la définition de leur intégration, par exemple en tant que personnes de référence. Les dynamiques qui lient ces quatre types de personnes varient en fonction du lieu, mais aussi des événements locaux, nationaux et internationaux. Dès lors, la question au cœur de notre projet est la suivante: comment les personnes concernées négocient-elles, construisent-elles et contestent-elles l'intégration? C'est ce que nous nous proposons d'examiner par le biais d'une perspective interdisciplinaire combinant sciences sociales et sciences juridiques.

\subsection{APPROCHER UNE RÉALITÉ COMPLEXE}

Notre but est double: d'un côté, nous souhaitons comprendre par quels processus des personnes étrangères sont considérées, en Suisse, comme suffisamment intégrées pour obtenir la nationalité de ce pays; de l'autre côté, nous voulons examiner l'impact de la notion juridique d'intégration dans le parcours de vie de ces personnes. Nous proposons de combiner une approche verticale et une approche horizontale. La première, verticale ou descendante, permet d'appréhender l'histoire de la notion d'intégration en Suisse telle qu'elle apparaît dans le corpus juridique, et telle qu'elle a été mise en œuvre. Nous visons ainsi à faire émerger les significations de la notion d'intégration modelées et développées au cours du $\mathrm{XX}^{\mathrm{e}}$ siècle dans l'ordre juridique suisse (chapitre 3), et la manière dont elles se déclinent dans l'ordre juridique actuel (chapitre 4). Le deuxième mouvement, horizontal, vise à rendre compte de la perspective des personnes étrangères elles-mêmes, comme actrices de leurs parcours de vie et de leur intégration dans la société. Fondé sur une analyse approfondie de cas empiriques et convoquant plusieurs disciplines, ce mouvement a pour ambition de donner 
voix aux personnes engagées dans des procédures administratives et légales. Ce faisant, il invite à retracer la complexité du processus d'intégration de leur point de vue (chapitres 6 et 7).

À la croisée de ces deux mouvements, notre recherche a mis en évidence le rôle incontournable des personnes travaillant dans les administrations cantonales et communales dont la tâche est d'appliquer la loi. Nous avons donc ajouté un chapitre qui analyse le travail de l'administration (chapitre 8). Ainsi, cet ouvrage se propose de créer un pont entre des personnes de diverses nationalités, avec des histoires de vie uniques, et les institutions suisses, qui, à travers le travail de leurs employées et employés, ont la tâche de traiter les demandes de naturalisation. Nous examinerons dans quelle mesure l'intégration peut être considérée comme un processus unilatéral et "positivisé", qui voit la personne étrangère comme destinataire passive de mesures étatiques, ou s'il ne s'agit pas plutôt d'un processus demandant à la personne étrangère un "agir" modulé en première personne; autrement dit, nous invitons à considérer cette personne comme participant activement au processus d'intégration et à l'évolution de celui-ci².

Les approches qui nous permettent de théoriser et d'analyser les diverses perspectives impliquées sont issues de diverses disciplines: l'analyse juridique ${ }^{3}$, l'approche socioclinique de l'impact de la loi dans la vie de ses destinataires, l'anthropologie des institutions et la psychologie socioculturelle. Nous présentons ci-après ces quatre approches.

\subsubsection{L'ANALYSE JURIDIQUE}

L'approche juridique part de l'hypothèse que l'ordre juridique national - la Constitution et/ou la loi -, en posant l'exigence d' "être intégré dans la communauté suisse» comme condition d'accès à la naturalisation (art. 14 let. a LN) ${ }^{4}$, impose une vision ou une

2. Pour une vision "active» du rôle des personnes étrangères dans la société d'accueil, voir Di Donato, 2012 b et 2020. Pour une référence ponctuelle à leur agentivité - entendue comme liberté de manœuvre vis-à-vis du système migratoire - et un développement de la thématique en termes historico-culturels, voir Garufo, 2015, p. 242.

3. Di Donato, 2016; Mahon et Collette, 2011.

4. Une révision totale de la loi sur la nationalité était en cours au moment où nous avons effectué les travaux menant à la rédaction du présent ouvrage. La loi fédérale sur la nationalité suisse (LN) du 20 juin 2014 a remplacé la loi du 29 septembre 1952 dès le $1^{\text {er }}$ janvier 2018. Comme notre projet de recherche et, surtout, les différents cas ou dossiers concrets - ou les parcours de vie - qui y sont traités se sont déroulés sous l'empire de l'ancienne loi (du 29 septembre 1952), en vigueur jusqu'au 31 décembre 2017, c'est en principe sur cette loi que notre ouvrage se fonde, tout en intégrant les nouveautés issues de la révision de 2014. 
représentation de ce que doit ou devrait être l'intégration. L'analyse juridique se fonde sur les méthodes traditionnelles de l'interprétation juridique, notamment l'interprétation historique, à l'aide de l'étude des travaux préparatoires (messages du Conseil fédéral, débats au sein du Parlement, etc.). Elle permet de déterminer, ou de reconstituer, la "volonté du législateur», c'est-à-dire l'idée ou la représentation que lui-même a ou avait d'une notion inscrite dans la loi, en l'occurrence celle d'intégration. Comme chacun le sait, le législateur n'a toutefois pas une volonté unique, univoque et claire; il est lui-même constitué de plusieurs personnes, provenant d'horizons socioculturels divers et d'orientations politiques différentes et, parfois même, antagonistes - c'est là le propre d'un régime démocratique et pluraliste. Le fruit de son action, la législation, n'est donc le plus souvent que le résultat d'un consensus, lui-même issu d'un ou de plusieurs compromis. La volonté du législateur - qui se manifeste à travers les notions et concepts qu'il utilise - n'est jamais entièrement univoque. Au contraire, derrière ces notions et concepts se cachent souvent des représentations plurielles et parfois contradictoires, qui permettent une «interprétation» de la loi, interprétation qui est elle-même une œuvre créative et qui se meut dans la "marge d'appréciation", justement, que les textes laissent à leurs interprètes.

C'est la raison pour laquelle l'analyse des textes juridiques, constitutionnels et/ou légaux, ne suffit généralement pas à reconstituer le sens des notions qu'ils emploient ou imposent. Il faut aussi examiner la manière dont ces textes et les notions qu'ils véhiculent sont appliqués, c'est-à-dire mis en ouvre, par les institutions et les personnes appelées et autorisées à les appliquer, à savoir l'administration ou, plus précisément, les administrations (fédérale, cantonales et communales). Cette mise en œuvre intervient essentiellement à travers des décisions qui touchent des personnes individuelles dans des situations concrètes, sur des demandes ou dans des procédures de naturalisation. Mais elle s'effectue aussi, souvent, à travers des directives édictées par les instances supérieures de l'administration, pour encadrer et faciliter le travail, et canaliser le pouvoir d'appréciation des instances inférieures et des personnes appelées à effectuer concrètement ce travail - les actrices et acteurs de l'administration qui rendent ou au moins préparent ces décisions.

Â côté du législateur et de l'administration, une troisième instance contribue elle aussi à forger les notions et concepts légaux, 
notamment la notion d'intégration qui nous intéresse: l'instance judiciaire. Au travers des recours dont elle est parfois saisie dans des cas individuels, généralement par les personnes directement concernées par une décision, l'autorité judiciaire est en effet amenée à donner son interprétation du sens qu'il faut attribuer à la notion d'intégration, interprétation qui devient dès lors - du moins lorsqu'il s'agit de la plus haute instance judiciaire (le Tribunal fédéral, en Suisse) - impérative pour les autres actrices et acteurs institutionnels (sauf pour le législateur, qui, lui, peut modifier la loi).

Lanalyse juridique a donc pour but de reconstituer, à travers l'étude de la genèse de la loi et à travers l'examen de son application par l'administration, et du contrôle de cette application par le juge (l'analyse de la jurisprudence), la signification que les actrices et acteurs institutionnels entendaient donner et donnent à la notion d'intégration. Cette analyse, effectuée dans le cadre de ce projet de recherche, a déjà fait l'objet d'une publication séparée, parue en 2016, en italien ${ }^{5}$. Dans les chapitres 3 et 4 , nous proposons donc une synthèse de ce travail en français.

\subsubsection{L'APPROCHE SOCIOCLINIQUE DU DROIT}

En complément de l'analyse juridique classique et son mouvement descendant, nous proposons ensuite une analyse qui va, cette fois, de bas en haut et qui examine le droit du point de vue des personnes qui en font l'expérience, considérées ici comme protagonistes de la construction du droit. L'approche socioclinique du droit nous permet ainsi de retracer le processus légal d'intégration non seulement à partir des lois et de leur mise en ouvre par des actrices et des acteurs institutionnels, mais aussi en rendant compte de la perspective des personnes de nationalité étrangère, candidates à la naturalisation, vis-à-vis du processus de création des significations juridiques.

À partir des années 1970, les études juridiques ont connu une importante réorientation, les faisant passer de l'analyse du droit dans une perspective légaliste ou formaliste - normalement fondée sur l'application mécaniciste des règles abstraites à des faits concrets - à une perspective sociologique et humaniste, qui prend en compte les représentations du droit de la part des personnes qui y sont confrontées ${ }^{6}$. Ce changement épistémologique est désigné comme le «tournant culturel», expression qui souligne le fait qu'il met le droit

5. Di Donato, 2016.

6. Ewick et Silbey, 1998; Merry, 2003; Silbey, 2008. 
en relation avec son contexte d'application ${ }^{7}$. Toujours en lien avec ce postulat du rôle actif des personnes directement concernées dans le processus de fabrication du droit $^{8}$, des orientations cliniques du droit se sont développées - d'abord dans le contexte des écoles de droit américaines ${ }^{9}$ et plus récemment en Europe ${ }^{10}$ et en Suisse ${ }^{11}$-, avec le double objectif de thématiser l'accès à la justice sociale de personnes vulnérables et de valoriser le rôle des "clients" en tant qu'actrices et acteurs dans la résolution de leur cas ${ }^{12}$.

Nous inspirant de ces orientations cliniques et sur la base des approches narratives de l'étude du droit ${ }^{13}$, nous proposons une perspective analytique centrée sur la personne, dans le but de comprendre son rôle dans son parcours administratif et légal et dans la construction de son histoire légale ${ }^{14}$. Ainsi, nous analyserons les cas juridiques du point de vue des personnes concernées par des décisions, et non du point de vue des administrations ou des juges (chapitres 6 et 7).

Depuis les années 1970, les chercheuses et les chercheurs en sciences sociales et en droit se sont également intéressés à comprendre les trajectoires individuelles des étrangères et des étrangers face au système migratoire selon différentes perspectives ${ }^{15}$, remettant en question les modèles fonctionnaliste et structuraliste, jusque-là dominants. Notre objectif est plus large; nous partons du principe que la personne étrangère, loin d'être uniquement à assimiler ou accoutumer (voir l'art. 14 let. a LN), est elle-même actrice de son parcours. Ainsi, des analyses de nombreux cas construits en collaboration avec les personnes concernées ont permis de montrer que l'acquisition progressive d'une conscience juridique les a parfois conduites à comprendre le fonctionnement du système légal et culturel au sens large, à accéder à l'administration et à la justice, et à contribuer à la modification des procédures dans le pays d'origine ou d'accueil ${ }^{16}$. C'est ce que nous appelons «l'agentivité légale ${ }^{17}$ (voir chapitre 7).

7. Gutwirth, 2013.

8. Latour, 2005.

9. Bloch, 2008, 2011; Wilson, 2017.

10. Bartoli, 2016; Di Donato et Scamardella, 2013; 2016; Di Donato, 2020.

11. Di Donato, 2018; Le Fort et Carron, 2016; Pribytkova, 2016.

12. Alfieri, 2016; López, 2017; White, 1997.

13. Amsterdam et Bruner, 2000; Binder et Weinsberg, 2000; Brooks, 1992; Bruner, 1991, 2003; Di Donato, 2020.

14. Di Donato, 2015.

15. Garufo, 2015; Kubal, 2013.

16. Di Donato, 2020.

17. Di Donato, 2014. 
L'approche socioclinique du droit complète donc l'analyse juridique classique (chapitres 3 et 4 ) en replaçant la loi dans la réalité de son contexte socioculturel d'application. Ce faisant, elle met en évidence le hiatus qui peut exister entre les visions du législateur, des administrations et des juges et leur impact dans la vie des personnes concernées (au niveau de leur vie quotidienne, de leur compréhension du système légal et des implications émotionnelles de cette compréhension).

\subsubsection{L'ANTHROPOLOGIE DES INSTITUTIONS}

L'anthropologie des institutions permet de jeter un éclairage critique sur la loi, en considérant les usages sociaux de celle-ci. En tant qu'elle produit de la différence entre deux groupes de personnes présentes sur le territoire national, les nationaux et les non-nationaux, la notion d'intégration peut être considérée comme un outil politique, légal et administratif ${ }^{18}$. Les personnes non nationales qui engagent une procédure de naturalisation doivent se soumettre à l'injonction de prouver qu'elles ont subi un "processus de transformation", contrainte à laquelle les Suisses échappent de facto (cf. chapitre 3).

Dans cette perspective, l'intégration, avec sa mise en scène et son évaluation, s'apparente à un acte d'institution à un double niveau. En premier lieu, elle met en évidence la manière dont les hiérarchies sociales s'organisent autour de distinctions institutionnalisées ${ }^{19}$. En reprenant l'analyse anthropologique classique des rites de passage, Turner ${ }^{20}$ a mis en évidence que la principale distinction produite par le rite d'institution n'oppose pas les personnes déjà initiées à celles qui ne sont pas encore adoubées, mais bien les personnes initiées à celles et ceux qui n'ont pas accès au rite et donc à la distinction sociale et au nouveau statut qu'il induit. De même, une fonction essentielle de l'intégration est de réaffirmer une communauté imaginée nationale ${ }^{21}$, celle des Suisses, en l'opposant à celles des non-Suisses. Cette tautologie permet d'échapper à la tâche impossible de définir précisément les critères communs à cette communauté. C'est l'affirmation que les autres sont différents de nous qui permet d'affirmer notre existence, et la rend légitime ${ }^{22}$.

18. Barth, 1969.

19. Bourdieu, 1982.

20. Turner, 1969.

21. Anderson, 2002 (1983).

22. Barth, 1969. 
En deuxième lieu, l'intégration est un acte d'institution dans la mesure où elle est mise en scène, recherchée, évaluée et attestée dans le cadre d'une procédure administrative. L'anthropologie des institutions offre donc un cadre d'analyse particulièrement pertinent, mettant au premier plan l'analyse des pratiques qui se déroulent en leur sein, des normes qui les structurent, ainsi que de ce qui en est dit; elle examine enfin les conséquences du travail des institutions pour les différents groupes sociaux impliqués. Elle permet notamment de disséquer les mécanismes institutionnels de prise de décision en incluant les divers critères, explicites ou tacites, mobilisés par les acteurs institutionnels. En ce sens, elle met en exergue le caractère à la fois contraint et contraignant de ces mécanismes: contraints dans la mesure où ils se déploient dans un cadre précis (le champ institutionnel dans lequel ils interviennent); contraignants, car ils régulent les actions des personnes qui sollicitent - ou sont en attente de - ces décisions ${ }^{23}$. En se centrant sur ces mécanismes et les diverses actions des professionnelles et professionnels de l'administration, l'anthropologie des institutions permet d'appréhender la marge de manœuvre, la part d'arbitraire, celle du soupçon s'attachant à des catégories de population, éléments qui interviennent dans leur travail, mais aussi de comprendre comment les règles sont négociées, contournées et interprétées.

L'anthropologie des institutions ne constitue pas un champ d'étude clairement balisé et défini. Apparue dans le contexte du rapatriement de l'anthropologie dans l'étude du proche ${ }^{24}$, elle côtoie et parfois recoupe les champs de l'anthropologie politique, de l'anthropologie de l'État et de l'anthropologie légale ${ }^{25}$. En l'occurrence, sa spécificité pour la présente recherche réside dans l'articulation de trois axes d'analyse: la question du lien entre des pratiques institutionnelles et un corpus de lois dont ces pratiques se réclament; les lieux et modalités du travail administratif propre à une ou plusieurs institutions; et l'ethnographie de ce travail par le biais de sa matérialité. Nous détaillons ces trois axes dans les paragraphes suivants.

Premièrement, ce sont les administrations qui sont responsables de la mise en pratique d'un corpus juridique (textes de loi, jurisprudence des tribunaux). L'anthropologie des institutions examine

23. Fossier et Monnet, 2009.

24. Aeby et Berthod, 2011.

25. Crozier et Friedberg, 2000 (1995). 
cette "vie sociale des lois ${ }^{26}$ ou la loi en tant que processus social, et ses effets concrets pour les personnes impliquées. Si de nombreuses études ont été faites sur la naturalisation en Suisse, tant en sociologie $^{27}$ et en anthropologie ${ }^{28}$ qu'en histoire ${ }^{29}$ et en droit ${ }^{30}$, la spécificité de cette approche réside en l'analyse minutieuse des processus produisant l'intégration, ainsi que de la dimension performative de l'usage de cette notion dans un contexte donné. Se centrant sur la marge d'interprétation et d'appréciation laissée aux autorités cantonales par la loi sur la nationalité ${ }^{31}$, l'approche que nous proposons examine la manière dont les représentantes et représentants de l'État «font» l'intégration et produisent des critères pour la mesurer. La mise en pratique de la loi suppose un traitement institutionnel fondé sur le va-et-vient entre cadre général et cas particuliers, entre les normes légales et les procédures permettant de les implémenter. Dans ce processus de mise en forme et de mise en sens, les actrices et acteurs institutionnels exercent leur marge de manœuvre, faisant appel à leur "feeling" ${ }^{32}$ et à des compétences générales (soft skills) acquises lors d'échanges avec des pairs sur les «bonnes" pratiques ${ }^{33}$. L'évaluation de la crédibilité joue un rôle central dans les relations entre État et potentiels bénéficiaires de prestations sociales ${ }^{34}$. Le soupçon institutionnalisé touche particulièrement celles et ceux qui recherchent un bénéfice ${ }^{35}$. L'acquisition de la nationalité helvétique apparaît ainsi construite comme un bien de prestige, un objet de désir à réguler et protéger ${ }^{36}$.

Le deuxième axe d'analyse de l'anthropologie des institutions concerne les modalités de la mise en application de la loi, en des lieux et des moments précis : les rencontres de guichet entre actrices et acteurs institutionnels, usagères et usagers ${ }^{37}$, ainsi que le traitement des dossiers dans les bureaux, comprenant l'évaluation des documents, de leur authenticité et de leur conformité aux attentes

26. Eckert, 2008; Nader, 2002.

27. Frauenfelder, 2007.

28. Par exemple, Centlivres et Schnapper, 1991; Ossipow, 1996, 2011.

29. Arlettaz, Argast et Studer, 2013; Studer, 2004.

30. Campisi, 2014; Di Donato, 2016; Gutzwiller, 2008.

31. CMR, 2015.

32. Hertz, Martin et Valli, 2004.

33. Lavanchy, 2013a, 2013b, 2014a; Maskens, 2015.

34. de Coulon, 2014; Eule, 2014.

35. Voir à ce sujet Laurens, 2008, sur la violence bureaucratique, et Graeber, 2012 et

2015, sur le pouvoir institutionnel.

36. Gutzwiller, 2008, pp. 121-141.

37. Spire, 2007, 2008; Spire et Fischer, 2009. 
de l'administration ${ }^{38}$. Les actrices et acteurs institutionnels ne sont en général pas des juristes, mais de fait des spécialistes de l'interprétation des textes de loi, et surtout de leur implémentation, qui font et défont les textes de loi au quotidien ${ }^{39}$; ce sont ainsi des «bureaucrates de rue ${ }^{40}$. Selon cette lecture, l'institution apparaît comme un espace de négociation et de confrontation, ainsi que de quête de pouvoir ${ }^{41}$. L'analyse des marges de manouvre de ces actrices et acteurs ${ }^{42}$ rend perceptible le hiatus entre ce qui est prescrit et les interprétations. Elle permet notamment de mettre en évidence l'articulation des services, ainsi que le rôle des différentes personnes, dans les prises de décision - la bureaucratie apparaît dans ce sens moins comme la réalisation d'un travail mécanique que comme une perpétuelle création et négociation de significations ${ }^{43}$, dans laquelle les actrices et acteurs institutionnels font preuve de réflexivité et de conscience face à leur propre pouvoir et à ses limites ${ }^{44}$.

Troisièmement, l'anthropologie des institutions s'intéresse à la matérialité de ce travail. En effet, les pratiques institutionnelles d'évaluation passent par la production de documents écrits, documents officiels - formulaires, copies de pièces d'identité, de certificats, etc. - et non officiels - annotations, échanges d'e-mails entre fonctionnaires, remarques griffonnées dans les marges, ajouts divers ${ }^{45}$. Si la matérialité du travail administratif est centrale, elle s'accompagne et se renforce par le biais de relations interindividuelles dans les bureaux. La discussion de cas problématiques, la demande d'aide ou de conseil ou le fait de donner son opinion contribuent à façonner des instruments de «droit mou» (soft law).

L'anthropologie des institutions permet donc d'examiner les pratiques sociales et normatives de l'intégration telles qu'elles se donnent à voir et sont générées par le travail de l'administration. Elle s'intéresse aux négociations de signification de la loi, à l'établissement de normes et aux interactions symboliques et matérielles dans les rencontres de guichet, concrètes ou médiatisées par des dossiers, entre le personnel administratif et les personnes qui présentent une demande de naturalisation.

38. Hull, 2012.

39. Fischer, 2007; Fischer et Darley, 2010.

40. Ou street-level bureaucrats (Lipsky, 1980).

41. Abélès, 1995.

42. Hertz et al., 2004.

43. Lavanchy, 2013a, 2013b, 2014a, 2014b, 2015.

44. Laurens, 2008.

45. Idem. 


\subsubsection{LA PSYCHOLOGIE SOCIOCULTURELLE}

La psychologie socioculturelle, ou culturelle, est une orientation de la psychologie contemporaine dont l'objet est l'être humain en tant qu'être de culture. Elle considère que chaque personne se développe de manière unique, mais que ce développement est guidé, rendu possible et contraint par l'environnement matériel, social et symbolique dans lequel la personne vit. La personne s'approprie activement des éléments culturels qui l'entourent, et son activité peut à son tour participer à sa transformation. L'approche met en outre l'accent sur les interactions entre la personne et son environnement social et culturel, sur les dynamiques de significations collectives et de sens individuel, rendues possibles par des dynamiques de médiation sémiotique ${ }^{46}$. Son intérêt pour l'articulation de l'individu et du collectif rapproche cette orientation d'autres approches de psychologie sociale ou de psychosociologie. C'est aussi de cette approche qu'est issue l'orientation narrative qui inspire les études sociocliniques du droit ${ }^{47}$ (1.2.2).

Une telle approche permet de porter une attention particulière aux trajectoires de vie des personnes qui s'engagent dans une procédure administrative ou légale. De ce point de vue, les systèmes juridique et administratif relèvent de l'environnement culturel dans lequel vivent les personnes. Ils font partie des contraintes et conditions dans lesquelles les personnes développent des projets de vie, peuvent en réaliser certains, rencontrent des obstacles qui les réorientent ou sont confrontées à des échecs. Comme dans les approches narratives du droit, il s'agit moins de comprendre si un témoignage ou une déclaration sont "vrais", ou si une décision est correcte, que de comprendre comment ceux-ci sont construits et négociés, à quoi ils répondent et ce qu'ils provoquent pour les personnes, en fonction de leur position dans le champ social. Cependant, la spécificité de la psychologie socioculturelle réside dans l'attention qu'elle accorde à la perspective unique de la personne, résultant de sa trajectoire de vie, et à partir de laquelle elle donne sens à la situation et s'engage dans différentes conduites $^{48}$. Comme l'approche socioclinique du droit, elle nous permet d'examiner en quoi une personne comprend, s'approprie ou résiste à la loi et l'administration qui la porte. En outre, et c'est là sa spécificité, en privilégiant une compréhension de la personne dans

46. Bruner, 1990, 1997, 2005; Cole, 1998; Valsiner, 2000, 2014; Valsiner et Rosa, 2007.

47. Amsterdam et Bruner, 2000.

48. Valsiner, 2014; Zittoun et al., 2013. 
sa totalité, cette approche nous invite à resituer les démarches liées à l'intégration dans le contexte plus global d'une trajectoire de vie.

\subsubsection{COMPLÉMENTARITÉ}

La rapide description des approches disciplinaires que nous avons convoquées donnera, nous l'espérons, une idée de la manière dont nous voyons leur complémentarité. Nous pensons en effet qu'elles apportent des éclairages différents à des processus complexes, qui se déroulent selon différentes temporalités, pris dans des enjeux spécifiques. Au chapitre 2, nous justifierons les prémisses épistémologiques qui permettent cette complémentarité. Ici, pour ne pas perdre davantage de temps, nous mettons d'emblée en évidence la manière dont nous concevons la fabrique de l'intégration.

\subsection{LA PROBLÉMATIQUE}

Le présent ouvrage a pour objectif de rendre compte de la manière dont la loi est appliquée et vécue par les différentes personnes - et, plus précisément, la loi sur la nationalité (LN), qui statue sur les conditions d'obtention de la nationalité suisse. Pour ce faire, nous adoptons une double lecture. D'abord, nous rendons compte de l'évolution historique de cette loi et de la naissance de la notion d'intégration dans le système juridique suisse, jusqu'aux discussions actuelles à son sujet ${ }^{49}$. Ensuite, en nous centrant sur les pratiques et sur les parcours de vie quotidienne, nous nous intéressons à des cas contemporains d'application de la loi. Pour cela, nous suivons de manière longitudinale les trajectoires administrativo-légales et personnelles de cinq personnes qui ont fait la démarche de demander la nationalité suisse. Nos analyses croisées se fondent essentiellement sur ces cinq études de cas, pour lesquelles nous disposons à la fois des dossiers administratifs et d'entretiens approfondis (nous faisons occasionnellement appel aux expériences de cinq autres personnes, dont l'une a fait une demande de renouvellement de permis, que nous avons seulement interviewées) (voir chapitre 2 pour la présentation des cas) ${ }^{50}$.

\section{Di Donato, 2016.}

50. Le projet de recherche initial envisageait d'inclure la loi sur les étrangers, qui contient aussi la notion d'intégration. Il s'agissait d'étudier les cas de personnes ayant fait une demande de prolongation de permis de séjour ou d'établissement ou de changement de permis. Nous avons fait ce travail de mise en parallèle des deux législations dans le cadre de l'analyse juridique - de la reconstruction de la notion d'intégration -, mais nous nous sommes concentrées, pour ce qui est de l'analyse des parcours de vie, sur le droit de la naturalisation. 
Pour rendre compte de ces trajectoires légales, nous proposons de considérer l'itinéraire type suivant: à un moment de sa trajectoire de vie, une personne, mue par des motifs qui lui sont propres, imaginant un certain avenir, entame les démarches visant à l'obtention de la nationalité suisse. Ce moment $\mathrm{T}$ marque le début de la procédure administrative. Celle-ci se clôt à un moment $\mathrm{T}+\mathrm{n}$, par la réponse donnée et reçue par la personne et le classement du dossier. L'approche que nous proposons nous permet alors de distinguer trois lignes de processus.

Premièrement, la demande de la personne donne lieu à la création d'un dossier qui entame une trajectoire administrative, dont la durée varie, dans les cas que nous avons analysés, entre quelques mois et une dizaine d'années, voire plus. Cette trajectoire est matérialisée par la circulation de documents écrits ou informatiques, les décisions de fonctionnaires, de représentantes et représentants de la loi et des institutions à différents échelons politico-administratifs, légaux et judiciaires. Elle s'achève lorsqu'une décision (qui semble définitive au moment où nous avons pris connaissance de ces dossiers) est prise et communiquée à la personne concernée.

En deuxième lieu, la trajectoire de vie poursuit, en parallèle, son évolution; la personne continue à travailler, à avoir des relations avec d'autres, à entamer des projets. À ce niveau, la personne fait aussi l'expérience de l'attente de la réponse à sa demande. Ses projets et son expérience de vie peuvent ainsi être plus ou moins marqués ou infléchis par la trajectoire administrative. L'expérience subjective et le sens que prend la démarche jouent donc ici un grand rôle.

Troisièmement, on observe les points de nouage de ces deux trajectoires lors de toutes les occasions de rencontre ou d'interaction entre la personne et le système administratif et légal; nous les appellerons des transactions. Celles-ci comprennent des rencontres face à face ou au guichet, ainsi que des lettres et des courriers écrits et des documents échangés entre les protagonistes. Il est à noter que, pour ces démarches, la personne doit exposer certains aspects de sa vie personnelle, mais qu'elle en protège d'autres. À ce niveau, on peut observer la manière dont la personne agit ou réagit face au système administratif et légal et discerner quelles stratégies elle met en place pour résoudre son cas. On parlera ici de pouvoir d'agir ou de l'agentivité légale de la personne ${ }^{51}$. 
Enfin, pour être tout à fait correctes, il nous faudrait ajouter une quatrième perspective, celle de nos regards de chercheuses et de chercheur sur le matériau auquel nous avons accès et que nous traitons en tant que données. Notre regard, ou, plus exactement, le faisceau de nos regards, est partiel, car nous ne pouvons décrire et analyser que les parties de la réalité qui se donnent à nous. De plus, malgré nos précautions méthodologiques et épistémologiques, nos perspectives sont toujours partiales, ou du moins situées. De cela nous rendrons compte aussi en faisant usage de nos réflexivités combinées; par souci de clarté, nous laisserons cependant ceci temporairement à l'écart.

\subsection{UN MODÈLE: ANALYSE DES TRAJECTOIRES D'INTÉGRATION}

Dans ce qui suit, nous reprenons et présentons de manière plus approfondie ces trois perspectives analytiques - la trajectoire administrativo-légale d'une demande, la trajectoire de vie de la personne et les transactions qui se jouent entre elles. Pour chacune, nous amenons des éléments théoriques qui nous permettent de problématiser notre objet. Sur cette base, nous formulons une série de sept hypothèses exploratoires qui auront pour fonction de guider notre analyse. Nous représentons ces trois perspectives dans le schéma suivant (figure 1).

\section{FIGURE 1 TRAJECTOIRES EN INTERACTION}

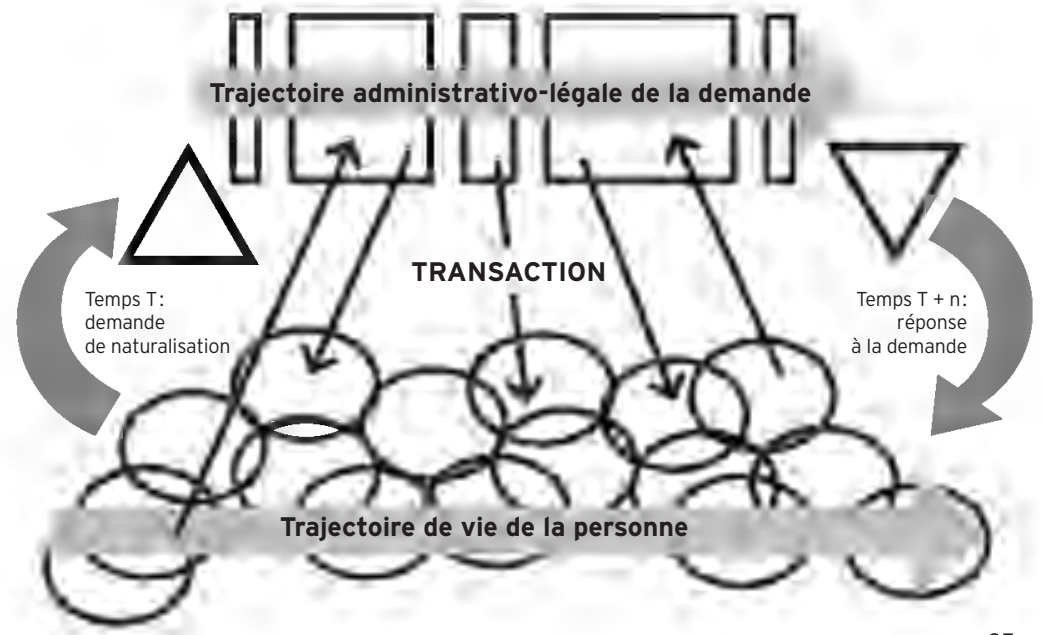


Le schéma présenté ci-dessus (figure 1) peut être lu de gauche à droite, en suivant une évolution temporelle. Il peut aussi être lu verticalement, suggérant à un moment donné $T$ + i la multiplicité des dynamiques qui constituent la réalité que nous étudions.

Dans le bas du schéma, nous avons représenté par une longue flèche la trajectoire de vie de la personne. La personne décide à un moment donné d'effectuer une démarche de demande de naturalisation. Elle remplit un formulaire et dépose une "demande de naturalisation" (triangle de gauche), initiant une procédure administrative. La procédure exige de rassembler au préalable toutes sortes de documents à réclamer auprès d'institutions suisses et non suisses, parfois difficiles à obtenir en raison de l'éloignement géographique, de l'organisation des services administratifs des pays tiers et des nomenclatures variables des documents officiels selon les contextes nationaux. Une fois que la demande est déposée, nous avons distingué, de manière analytique, trois niveaux de phénomènes: la trajectoire administrativo-légale, la trajectoire de vie de la personne et les transactions qui les nouent.

\subsubsection{LA TRAJECTOIRE ADMINISTRATIVO-LÉGALE}

L'enchaînement supérieur de rectangles représente les étapes de la procédure administrativo-légale initiée au moment de la demande de naturalisation, incluant des actrices et acteurs institutionnels aux niveaux fédéral, cantonal et communal - fonctionnaires, personnes conseils, avocates et avocats, voire instances judiciaires, comme nous le montrerons (voir chapitre 5). Notons aussi que les procédures varient selon les cantons, et que les étapes dont nous parlons sont celles du canton de Neuchâtel, que nous avons étudié.

Au niveau théorique, cet enchaînement d'actrices et d'acteurs administratifs et légaux représente «l'institution». Une institution peut être définie comme "tout système de croyances ou mode collectif d'action " ${ }^{52}$ auquel sont en principe associés des mécanismes de régulation ou de sanction ${ }^{53}$. Il existe de très nombreux types d'institutions ${ }^{54}$ : certaines sont liées à des organisations spécifiques en des lieux géographiques concrets, d'autres sont plus informelles, délocalisées ou virtuelles; elles peuvent aussi être de tailles très diverses - on parle par exemple de l'institution du mariage ou de

52. Durkheim, 2002, p. 15.

53. Henning, 2007.

54. Berthod et Aeby, 2011. 
celle des Nations unies. Les chercheuses et chercheurs en sciences sociales s'intéressent particulièrement aux institutions dites totales, qui régulent tous les aspects de la vie d'une personne, comme une prison ou un hôpital psychiatrique ${ }^{55}$. Toutefois, pour mieux rendre compte de la modernité, la réflexion s'est étendue à des institutions plus fluides ${ }^{56}$. En particulier, Susie Scott a récemment proposé la notion d' «institution de réinvention " pour désigner les institutions dont la fonction principale est de transformer le statut identitaire de personnes qui y entrent en principe de manière consentante ${ }^{57}$, comme certaines organisations religieuses, des écoles ou des sites d'entraide. Bien que l'institution qui nous intéresse ne soit pas limitée à un bureau ou une organisation, mais relève d'un réseau d'offices et d'acteurs sociaux communaux, cantonaux et fédéraux, il semble intéressant de faire le rapprochement. En effet, la procédure qui vise à l'application de la loi sur la nationalité peut aussi être vue comme une structure matérielle, sociale, discursive et symbolique dont la fonction est de guider, évaluer et valider une transformation statutaire des personnes qui initient la démarche. C'est cette idée que nous allons décomposer dans les sept hypothèses qui suivent.

Notre première hypothèse exploratoire est que les procédures de naturalisation se sont progressivement transformées de simples démarches de validation de la légitimité d'une demande (octroi automatique de la nationalité ou "incorporation » par droit du sol) vers des procédures de transformation de la personne (par le biais de l'exigence d' "assimilation» et, plus tard, d' «intégration»). C'est d'ailleurs ce que postule la lettre de la loi sur la nationalité de 1952, qui demande aux étrangères et aux étrangers de se familiariser et de s'accoutumer "au mode de vie et aux usages suisses" (art. 14 let. b LN). Le chapitre 3 reconstruit cette évolution et le chapitre 4 examine de manière plus synchronique ce que contient la notion d'intégration aujourd'hui en Suisse. Il s'agira également d'observer les dynamiques par lesquelles ces opérations de transformation sont mises en place au niveau législatif, sur le fond (chapitre 4) et sur le plan de la procédure (chapitre 5).

Une institution de réinvention a sa propre logique et n'est pas toujours et primairement au service du projet des personnes. Dans le cas qui nous intéresse, l'institution est constituée d'un très grand

55. Goffman, 1961; Scott, 2011

56. Bauman, 2000; Berger et Luckmann, 2010.

57. Scott, 2011, p. 3. 
nombre d'offices et de professionnels, dans un tissu social, politique et économique non homogène et dynamique. Les hiérarchies produisent des valeurs, des instructions et des recommandations qui guident alors le travail des employées et employés de l'administration en charge des dossiers de naturalisation. Ces instructions sont à la fois des moyens de bien faire leur travail et des garde-fous contre la charge psychologique que confere le pouvoir de décider de la trajectoire de vie d'autrui; elles aussi ont l'effet très particulier de déresponsabiliser le personnel de l'administration. Toutefois, étant donné la complexité d'une institution comme celle qui nous intéresse, ces instructions et ces valeurs peuvent être contradictoires entre elles, et en porte-à-faux avec la sensibilité des personnes en charge de leur réalisation. Il en résulte que le travail de cette institution de réinvention peut être au service d'objectifs implicites et explicites divergents (assurer un traitement équitable tout en respectant l'égalité, être généreux sans brader la nationalité, etc.), et parfois très éloignés de ce qui guide les demandes de la personne. Notre deuxième hypothèse exploratoire est que l'application de la loi visant à vérifier l'intégration d'une personne se traduit par une pratique dont les objectifs sont nombreux et parfois contradictoires et dont la mise en œuvre se noue dans un jeu de déterminations multiples et complexes. Il s'agira donc de comprendre, d'un côté, comment les actrices et les acteurs institutionnels construisent leurs dossiers et prennent leurs décisions, ce qui sera l'objet en particulier des chapitres 4, 5 et 8. De l'autre côté, il s'agira d'analyser la manière dont les personnes concernées par l'exigence d'intégration réagissent vis-à-vis des pratiques administratives et légales, ce qui sera l'objet du chapitre 7.

L'institution ne fait ainsi pas que de répondre à une demande: elle obéit à des impératifs et à sa logique ${ }^{58}$; elle a sa propre temporalité, parfois ralentie par une forme d'inertie et, à d'autres moments, saisie d'accélérations. En conséquence, l'entrée d'une personne dans une démarche administrative d'une durée de plusieurs mois, voire de plusieurs années, n'est pas anodine; elle implique que la personne devra se soumettre à cette logique spécifique. En effet, la procédure produit des traces matérielles, souvent des documents, qui circulent d'une personne à l'autre dans les administrations, et qui ont des effets: ces traces peuvent être stigmatisantes (elles

58. Mehan, Hertweck et Meihls, 1986. 
catégorisent négativement les personnes au sujet desquelles elles sont produites), performatives (elles créent de la réalité, notamment le soupçon) et déterminantes (elles orientent ou infléchissent des trajectoires de vie). La façon de créer et agencer un dossier administratif lors de la procédure a une incidence sur la trajectoire de la demande. Le dossier n'est pas qu'un instrument neutre utilisé par des institutions, il sélectionne, modifie et réagence des informations ${ }^{59}$, devient un médiateur, voire un acteur non humain de la prise de décision administrative ${ }^{60}$. Notre troisième hypothèse exploratoire est ainsi que le travail de l'administration est une entreprise de production de sens et de légitimité qui se traduit notamment par la production de traces lexicales et matérielles. Les effets de celles-ci sont performatifs et peuvent jouer un rôle majeur dans l'évaluation d'une demande et la prise de décision, voire décisif dans les débouchés de la démarche administrative. De cette manière, l'institution contribue à transformer la signification sociale d'une demande (voir chapitre 8).

\subsubsection{LA TRAJECTOIRE DE VIE}

Il nous faut donc à présent considérer la trajectoire de la personne qui a déposé sa demande. Constituée d'une pluralité de sphères d'expérience, sa vie se poursuit, mais est maintenant en partie exposée au regard et au jugement de l'institution de réinvention. La notion de sphère d'expérience ${ }^{61}$, en suivant notamment Kurt Lewin ou Alfred Schuetz, désigne la diversité d'expériences cohérentes et socialement situées que traverse une personne - des expériences qui engagent certaines activités, aspects identitaires, motifs et formes de reconnaissance, et que la personne vit comme similaires et allant de soi. Au cours d'une trajectoire de vie, certaines sphères coexistent à un moment donné, certaines disparaissent et d'autres apparaissent: l'école primaire, la vie dans un village, un repas en famille, le travail à la pizzeria ou l'entraînement de foot.

La personne qui dépose une demande de naturalisation arrive donc à un moment donné de sa vie, avec des sphères d'expériences passées qui ont précédé et parfois déterminé des sphères actuelles, et qui participent en particulier à la demande de naturalisation. La demande de naturalisation peut être lue comme étant soutenue

59. Hull, 2012.

60. Latour, 2005.

61. Zittoun, 2012; Zittoun et Gillespie, 2015, 2016. 
par - ou traduisant - ce que l'on appelle couramment un projet: la personne imagine que, au regard de son expérience jusqu'ici, elle est en droit de, ou elle pourrait être reconnue comme apte à, ou elle serait intéressée à "devenir suisse" ${ }^{62}$. En termes plus théoriques, on pourrait dire que, aux sphères d'expériences proximales de la personne en famille ou au travail s'ajoutent des sphères d'expérience distales que développe la personne, sphères détachées de l'expérience ici et maintenant socialement partagée ${ }^{63}$. Ici, ces sphères distales réunissent ainsi à la fois des souvenirs d'expériences passées - le souvenir d'un chez-soi précédent, perdu à cause de l'exil, ou celui d'une enfance en Suisse - et des expériences imaginaires à venir - le rêve d'une vie facilitée, le projet d'une reconnaissance sociale de son appartenance, de pouvoir voter, de voyager plus facilement ou d'offrir plus de sécurité à ses enfants. Ainsi, concernant cette trajectoire, notre quatrième hypothèse exploratoire nous invite à examiner combien la demande de naturalisation correspond à une ou des imaginations de soi, et a, dans ce sens, des implications affectives et identitaires dans différentes sphères d'expérience (chapitre 6).

Une fois la demande déposée et la trajectoire administrative initiée, la vie de la personne se poursuit, et nous avons représenté cela par la flèche du bas de la figure 1, sur fond d'ovales représentant ses sphères d'expérience. Pour une part, cette trajectoire de vie se déroule indépendamment de la trajectoire administrative. Pour une autre part, elle est ponctuée par celle-ci, lorsque la personne reçoit des demandes de documents complémentaires, doit répondre à des questions ou payer des émoluments liés à la démarche. En conséquence, trajectoire administrative et trajectoire personnelle nouent des relations complexes et sont étroitement liées.

Ainsi, une personne qui fait une demande alors qu'elle est mariée peut voir son état civil changer en cas de divorce ou de décès durant le traitement de la demande; ou encore, elle peut changer d'emploi ou de situation financière, ce qui a des implications sur le traitement de la demande. À ce niveau, nous nous intéressons à la subjectivité de la personne; nous pouvons en partie reconstruire la manière dont la trajectoire administrative affecte la personne, lorsque par exemple sa parole a été mise en doute dans le traitement de son dossier, ou qu'elle trouve que les questions posées mettent

62. Centlivres, 1991.

63. Zittoun et de Saint-Laurent, 2015; Zittoun et Gillespie, 2016. 
en cause la légitimité de sa demande et celle de sa présence sur le territoire, ou lorsque des obstacles apparaissent. Les questions qui surgissent lors du traitement administratif, mais s'étendent au-delà de la sphère bureaucratique, peuvent affecter la mise en récit de soi, les émotions et l'identité.

Enfin, nous avons indiqué par un deuxième triangle, cette fois inversé, la fin présumée de la procédure - la réponse à la demande initiale. L'histoire ne s'arrête pas là, et nous avons représenté les sphères d'expérience de la personne après la réponse: la trajectoire de vie de la personne se poursuit, mais qu'est-il arrivé à son projet, comment la réponse reçue affecte-t-elle ce qu'elle imagine pouvoir faire de sa vie?

En conséquence, la cinquième hypothèse exploratoire que nous formulons est que la trajectoire de la personne est constamment transformée par la trajectoire administrative, et donc que les sphères d'expérience seront d'autant reconfigurées - en particulier parce que la trajectoire administrative a pu amener à des expériences qui ne sont pas consonantes avec le projet de vie. La manière dont la demande de naturalisation peut refléter une imagination de soi et la manière dont la procédure peut affecter une trajectoire de vie sont traitées dans le chapitre 6.

\subsubsection{LES TRANSACTIONS}

Nous avons voulu aussi mettre en évidence les relations, ou transactions, entre la trajectoire de vie et la trajectoire administrativo-légale ${ }^{64}$, transactions que nous avons représentées par des flèches diagonales dans notre schéma. Nous identifions deux types de transactions. Les premières résultent d'échanges explicites, directs ou médiatisés par courrier, entre la personne requérante et l'administration. Ces transactions sont susceptibles d'accélérer, de ralentir et d'infléchir les trajectoires elles-mêmes. Le deuxième type de transactions regroupe des dynamiques ou des pratiques qui sont mises en place par les personnes requérantes ou l'administration, pas explicitement adressées à l'autre, parfois même invisibles, et qui peuvent influer sur la procédure.

Parmi les transactions explicites, nous devons en particulier mettre en évidence les interactions réelles entre la personne candidate et le personnel de l'administration. Ces interactions ont lieu

64. Dewey, 1946. 
dans des espaces sociaux partagés (le guichet de l'administration, un bureau, etc.) et deviennent sphères d'expérience subjectives pour les personnes - celle qui fait la demande comme pour son vis-à-vis institutionnel. Ces interactions sont l'occasion de négociations, d'évaluations mutuelles, de constructions de sens croisées ou communes. À ce niveau, on assiste donc à la loi en train de se faire. Les personnes ne sont pas simplement soumises aux aléas d'une procédure qui leur échappe; elles peuvent aussi au contraire engager leur agentivité légale ${ }^{65}$, leur pouvoir d'agir face à la loi ou aux démarches administrativo-légales. Évidemment, toutes les personnes déposant une demande ne sont pas égales entre elles; certaines ont une formation qui leur donne accès à des compétences utiles, certaines vont chercher de l'aide auprès d'associations, d'amis, de soutiens politiques ou, si elles peuvent se le permettre, d'avocates ou d'avocats; elles font ainsi usage de différentes ressources qu'elles utilisent dans leurs échanges avec l'administration. Nous pouvons alors formuler une sixième hypothèse exploratoire, qui nous invite à examiner le rôle de l'agentivité légale de la personne dans l'évolution de la trajectoire administrative; c'est l'objet du chapitre 7.

Ensuite, les trajectoires administratives et de la personne ne sont pas transparentes l'une à l'autre, ce qui génère des transactions implicites. D'une part, chaque partie cherche à connaître ou comprendre l'autre et, en même temps, peut chercher à cacher ou dissimuler certains aspects la concernant. Ainsi, la personne qui fait la demande peut chercher à cacher certaines sphères d'expérience qui, selon elle - ou d'après ses représentations des attentes de l'administration - mettraient en doute la légitimité de sa demande (par exemple, un incident avec des voisins), tout en mettant en évidence d'autres aspects (par exemple, un engagement associatif). D'autre part, l'administration a parfois accès à certaines sphères d'expérience de la personne, alors que celle-ci l'ignore - par exemple, une vieille dette. Inversement, l'administration peut plus ou moins dévoiler les mécanismes de prise de décision, en incluant certains courriers électroniques imprimés ou en omettant des remarques sur des Post-it ou des annotations à la main prises lors d'une conversation téléphonique, par exemple au sujet d'un rapport de police ou de l'évaluation d'une personne de référence.

65. Di Donato, 2014 et 2020. 
Il s'agit donc d'un périlleux équilibre entre dévoilement et sauvegarde de l'intime, tous deux faisant l'objet d'injonctions sociales contradictoires, ou du moins en tension. Notre septième hypothèse exploratoire nous invite donc à examiner ces transactions et, en particulier, nous amène à considérer dans quelle mesure la trajectoire administrative peut être guidée par des dynamiques souvent ignorées par les personnes requérantes; la question est traitée au chapitre 8.

$\mathrm{Au}$ total, donc, nous proposons de montrer que la procédure de naturalisation est progressivement devenue une institution de réinvention, et que le résultat du nouage des trajectoires administrative et personnelle est une transformation de la personne et de sa trajectoire de vie. En quoi ces transformations aboutissent à «intégrer» ou "désintégrer» la personne est ce que nous explorerons en conclusion de cet ouvrage.

\subsection{SYNTHÈSE ET PLAN DE L'OUVRAGE}

Nous proposons donc ici une analyse historique et interdisciplinaire de la notion légale d'intégration en Suisse. Nous observons tant l'émergence et la définition progressive de cette notion dans le corpus légal que les conséquences concrètes de son application, du point de vue tant de ceux qui veillent à la mise en place de la loi au niveau administratif que de ceux qui sont exposés à l'évaluation de leur vie à la lumière de cette loi.

Cet ouvrage est ainsi construit en quatre parties. La première est introduite par le présent chapitre (chapitre 1), qui pose le cadre théorique et la problématique. Elle se poursuit par une présentation de la méthodologie (chapitre 2). La deuxième partie décrit le contexte institutionnel de la notion d'intégration en Suisse. Le chapitre 3 en retrace l'apparition et l'évolution dans l'ordre juridique suisse dans une perspective historique. Le chapitre 4 analyse la signification de la notion dans la pratique administrative et la jurisprudence actuelles. Le chapitre 5 explicite la procédure et les diverses étapes administratives par lesquelles transitent les demandes de naturalisation en Suisse, et qui engagent le travail de la commune, du canton et de la Confédération. La troisième partie, qui s'inscrit dans une perspective analytique, rend compte des trois trajectoires identifiées plus haut. Le chapitre 6 se centre sur les trajectoires de vie des personnes dont l'intégration est mise 
en examen et sur les conséquences subjectives de l'expérience du travail de l'administration. Le chapitre 7 reconstruit les trajectoires administratives et légales des personnes requérantes et montre la manière dont elles s'engagent activement face à loi, manifestant leur agentivité légale. Centré sur le travail des professionnelles et des professionnels de l'administration, le chapitre 8 comprend tant l'analyse de la matérialisation de la demande par le dossier que celle des processus de prise de décision. La dernière partie de ce livre, conclusive, sera l'occasion de revenir sur nos questions initiales et les hypothèses qui ont guidé notre exploration. En particulier, nous pourrons alors réexaminer la notion d'intégration et nous demander si, en fin de compte, elle désigne un processus ou son aboutissement. Nous mettrons aussi en évidence quelques recommandations auxquelles ce travail nous a menées tant pour la recherche que pour la pratique. 


\title{
2. FABRIQUER LA RECHERCHE: MÉTHODOLOGIE
}

ANNE LAVANCHY, FLORA DI DONATO, ÉLODIE GARROS, PASCAL MAHON ET TANIA ZITTOUN

\begin{abstract}
e chapitre poursuit deux objectifs. Le premier, descriptif, détaille les démarches effectuées par l'équipe, le recrutement des participantes et participants, le type de données produites lors du terrain et la manière dont elles ont été analysées. Le second met en lumière les enjeux de l'intégration en revenant sur le positionnement de l'équipe de recherche et les questions éthiques soulevées tant par la thématique que par les choix méthodologiques qui ont été faits. Pour ce faire, nous débutons par la présentation d'une méthodologie façonnée par l'interdisciplinarité et la démarche qualitative (2.1). Suit une présentation du travail de terrain (2.2), des personnes qui ont participé à la recherche, à savoir les candidates et candidats à la naturalisation (2.3), puis celles travaillant dans les institutions administratives et politiques concernées (2.4). Le chapitre se clôt sur la description du processus qui a permis de reconstruire des cas (2.5), et celle des enjeux éthiques de la méthodologie adoptée (2.6), qui aborde la question des impacts de la recherche et explicite les positionnements de l'équipe et de ses membres.
\end{abstract}

\subsection{UNE MÉTHODOLOGIE QUALITATIVE INTERDISCIPLINAIRE}

L'équipe de recherche a mis sur pied une méthodologie originale, fondée sur nos connaissances disciplinaires respectives. Il s'est agi de cerner et d'expliciter les attentes individuelles, de les collectiviser et de construire à partir de ces différents apports une méthodologie permettant de remplir nos objectifs analytiques.

D'emblée, un des principaux atouts de la recherche était de faire entrer en dialogue des perspectives juridique, sociojuridique, psychologique et anthropologique sur un objet commun, l'intégration. Constituée de chercheuses et chercheur ayant des expertises en droit 
constitutionnel, en droit public, en psychologie socioculturelle, en philosophie et sociologie clinique du droit et en anthropologie des institutions, l'équipe a travaillé à constituer un bagage commun à deux niveaux. D'une part, nous avons collectivisé des concepts clés pour chacun de ses membres; d'autre part, nous avons identifié le type de données nécessaires à ces approches, la manière de les produire et de les analyser.

Pour atteindre nos objectifs, nous avons dû définir des modalités de travail collaboratif. Concrètement, les rencontres d'une journée rassemblant toute l'équipe ont jalonné la recherche. S'y sont ajoutées des séances à deux ou trois, à géométrie variable selon les besoins de chaque étape: d'abord pour constituer des grilles d'entretien, organiser et réaliser le travail de terrain; puis pour rendre compte des entretiens et ébaucher des axes d'analyse pertinents; enfin, pour construire les analyses dont les chapitres du présent ouvrage rendent compte. L'ensemble des textes produits de manière personnelle ou collective a circulé et bénéficié des commentaires de toute l'équipe.

Le dialogue sur les conditions de recherche et la production des données significatives a représenté un investissement conséquent en temps et en énergie, notamment pour expliciter les impératifs méthodologiques respectifs, le type de documents nécessaires à une reconstruction des cas d'un point de vue juridique, le rôle des entretiens et les éléments à aborder avec les personnes participantes. L'objectif des entretiens devait être, d'une part, de comprendre les ressources et l'agentivité des candidates et candidats face à la procédure spécifique entamée, mais aussi, d'autre part, de fournir des données sur leurs trajectoires biographiques et la manière dont la procédure les a modifiées ou non.

La conséquence de cet investissement collectif a été de revoir à la baisse l'ampleur de la recherche. Initialement conçue dans une perspective pluricantonale, elle a finalement été centrée sur un seul canton, ce qui a permis de construire des cas de manière plus approfondie et d'enrichir leur interprétation en incluant les agents de l'État aux côtés des personnes ayant effectué une démarche de naturalisation ou de renouvellement de permis. En analysant les différentes conceptions de l'intégration, leur rencontre, ainsi que la matérialisation et la visualisation, par divers documents, du travail d'appréciation de l'administration, cet ouvrage vise à offrir un tableau à la fois complet et nuancé des modalités de négociation de l'intégration. Il rend compte des antagonismes entre les sens variés 
qui lui sont attribués, des rapports de pouvoir, des ressources et compétences d'agentivité et des marges de manœuvre.

\subsubsection{LE TRAVAIL COLLABORATIF}

Le travail collectif d'élaboration a d'abord consisté à clarifier les concepts en construisant un vocabulaire commun et en débusquant les "faux amis»- des termes similaires dont les usages et les implications théoriques et méthodologiques varient d'une discipline à l'autre. En explicitant ces concepts et leur histoire, nous avons travaillé à harmoniser notre posture. Cependant, le texte issu d'une longue collaboration porte les échos de nos diverses voix, ce qui ouvre de nouvelles perspectives et permet d'enrichir les différents champs de savoir mis à contribution. Tous les chapitres ont été relus et commentés, à plusieurs reprises, par l'ensemble de l'équipe de recherche. Cette recherche collaborative peut ainsi être définie comme produite par des «interactions institutionnellement situées entre des perspectives divergentes avec un but (partiellement) partagé [et] rassemblant des perspectives divergentes sur une question ou un objet d'intérêt ou de connaissance commun ${ }^{1}$. Un tel travail requiert une triangulation des perspectives qui permet de construire un objet de manière plus riche ou plus complexe qu'il ne le serait dans une seule perspective ${ }^{2}$. Sous certaines conditions ${ }^{3}$, il présente l'avantage de gagner en réflexivité (notamment sur les implicites de chacune des disciplines) et, idéalement, de conduire à l'émergence d'idées et de connaissances nouvelles - soit sur l'objet de savoir, soit sur la pratique même de la collaboration ${ }^{4}$. Nous avons aussi suggéré que les projets collaboratifs peuvent varier sur les dimensions suivantes: selon que les chercheuses et chercheurs admettent ou non des présupposés de base; qu'il y a une répartition claire des tâches, ou une superposition de celles-ci; que les collaboratrices et collaborateurs se connaissent bien ou non; que le pouvoir décisionnel est centralisé ou partagé; et que les buts sont communs ou divergents ${ }^{5}$. Dans ce projet, où nous avons travaillé à partir de disciplines différentes, avec une répartition relativement

1. Cornish, Zittoun et Gillespie, 2007, p. 18, notre traduction.

2. Flick, 1992.

3. Notamment, pour être porteur, le travail collaboratif doit éviter les risques de la dissolution de responsabilité, et respecter les besoins de la créativité individuelle - notamment en temps et en espace d'exploration (Toomela, 2007).

4. Zittoun, Baucal, Cornish et Gillespie, 2007.

5. Cornish et al., 2007, p. 29; Zittoun et al., 2007. 
souple des tâches et une autorité distribuée, il a été important de définir un but et des présupposés communs.

\subsubsection{LES PRÉSUPPOSÉS ÉPISTÉMOLOGIQUES}

Les approches qui sont les nôtres - l'analyse juridique, l'approche socioclinique du droit, l'anthropologie des institutions et la psychologie socioculturelle - travaillent à partir de certains présupposés épistémologiques que nous distinguons à des fins analytiques, mais qui sont de fait mutuellement dépendants.

Premièrement, ces disciplines admettent toutes l'historicité des phénomènes étudiés, c'est-à-dire leur nature temporelle et dynamique, tout en les déclinant différemment. Au niveau juridique, on admet que les lois sont le produit de négociations et de constructions sociohistoriques ${ }^{6}$ et que la jurisprudence elle-même crée sa propre histoire. On peut alors parler de sociogenèse, c'est-à-dire de la création d'une réalité sociale partagée. En anthropologie, la réalité sociale se négocie dans les interactions situées et se construit par les pratiques. L'approche socioclinique examine quant à elle la manière dont la réalité légale se construit par les récits ${ }^{7}$, récits qui sont coproduits par les institutions légales et par les personnes qui vivent cette réalité ${ }^{8}$. Enfin, la psychologie socioculturelle s'intéresse aux trajectoires de vie des personnes et à la manière dont celles-ci se développent au travers de ces microgenèses, et l'on peut parler d'ontogenèse 9 . Ces approches s'intéressent à une réalité mouvante, évoluant dans un temps irréversible, mais qui crée des temporalités spécifiques pour des acteurs et des actrices qui ont leurs propres finalités et positionnements. Ainsi, une interaction administrative de quelques minutes peut donner lieu à un document visant à l'obtention d'un droit qui va circuler pendant une année parmi différents services administratifs, avant d'être envoyé à la personne requérante, dont la vie a pu, dans ce laps de temps, connaître des changements majeurs.

Deuxièmement, nos disciplines admettent toutes la nature socialement construite, et donc polyphonique, de la réalité ${ }^{10}$. En ce sens, cette réalité est non seulement négociée entre personnes,

\footnotetext{
6. Cover, 1983-1984; Studer, Arlettaz, Argast, 2013.

7. Sherwin, 1993.

8. Di Donato, 2008a, 2011, 2015a.

9. Duveen et Lloyd, 1990; Gillespie et Cornish, 2010.

10. Berger et Luckmann, 1966.
} 
au travers de discours verbaux - oraux et/ou écrits ${ }^{11}$ - et au travers de mouvements et de formes non verbales, mais elle est aussi constituée d'actrices et acteurs "non humains", et en particulier de dispositifs matériels et techniques: des locaux, des meubles, des guichets, des documents, des cartes d'identité, des rues et des villes ${ }^{12}$. En revanche, nous admettons la réalité ontologique de ces actrices et de ces acteurs du social.

Troisièmement, le travail interdisciplinaire nous invite à une approche "perspectivale», c'est-à-dire attentive aux perspectives spécifiques des actrices et acteurs sur un objet donné. Ces perspectives orientent les actions vers certaines finalités, guidées par certaines valeurs, ou attentes en fonction de leurs positions dans le champ social. Celui-ci est lui-même non neutre, parcouru de jeux de forces qui rendent ces positions inégales (en termes de légitimité, de pouvoir d'agir, de moyens économiques, etc.) et donc attribuent des pouvoirs asymétriques aux actrices et acteurs sociaux. Toute interaction (réelle ou virtuelle) est dans ce sens une rencontre de perspectives, et toute analyse de la réalité demande de prêter une attention particulière à ces perspectives ${ }^{13}$. De cela découlent différentes implications, notamment sur la nature normative de la réalité - normes fixées par certains et subies par d'autres ${ }^{14}-$ ou encore sur les décalages entre les significations partagées de certains objets sociaux et le sens qu'ils peuvent prendre pour chacune des personnes, en fonction de sa trajectoire biographique ${ }^{15}$.

Enfin, quatrièmement, nos regards croisés sur un objet hautement normatif - l'application de la loi - nous invitent à adopter une perspective pragmatiste sur la réalité observée ${ }^{16}$. Celle-ci considère comme pertinents des objets ou des idées qui ont un effet sur la réalité: des concepts qui nous permettent de voir la réalité autrement (de manière plus riche ou nuancée) ou encore des objets ou des idées qui font faire des choses particulières aux actrices et acteurs que nous observons. Dans tous les cas, l'usage de telle idée ou de tel objet affecte la réalité des personnes concernées. Concrètement, cela nous rend attentives aux notions, valeurs ou

11. Voir, par exemple, les travaux sur les pratiques d'écriture en milieu professionnel (Borzeix et Fraenkel, 2001; Sociologie au travail, 2014, vol. 56) et les travaux sur l'agentivité textuelle (Fraenkel, 2006).

12. Brinkmann, 2006; Latour, 2005.

13. Gillespie, 2006b; Martin et Gillespie, 2010; Mead, 1934.

14. Brinkmann, 2010.

15. Leontiev, 1996; Rochex, 1998.

16. Brinkmann, 2004; James, 1904. 
pratiques qui font faire des choses aux gens, ou dont les gens font usage dans certaines circonstances, et à leurs résultats, qu'ils soient intentionnels ou non.

En résumé, au-delà des différences de traditions disciplinaires, nous avons travaillé sur la base de cette épistémologie commune, admettant la nature historique d'une réalité que nous considérons comme le fruit de négociations entre des perspectives multiples d'actrices et acteurs orientés. Notre approche est dialogique, en ce sens qu' elle considère l'ancrage social et culturel des actes sociaux et communicationnels, leur position et leur orientation vers, ou leur adresse à, un autre ${ }^{17}$.

\subsubsection{UNE DÉMARCHE QUALITATIVE}

Une démarche de type qualitatif s'est révélée la plus à même de prendre en considération les spécificités et les difficultés de l'objet d'étude choisi, à savoir appréhender les interprétations et impacts de la notion d'intégration dans les trajectoires des candidates et candidats ${ }^{18}$. Le but n'était pas de travailler dans une optique statistique, en établissant par exemple un échantillon représentatif de la population qui aurait entamé une procédure impliquant l'évaluation de l'intégration. Comme la recherche accordait d'emblée une place importante à la perspective et l'agentivité des candidates et candidats (voir chapitres 6 et 7), elle était tributaire de leur disponibilité et de leur volonté d'y participer.

Des données denses ${ }^{19}$ fondées sur un nombre restreint de cas permettent d'amener une analyse plus générale dépassant les simples expériences particulières ${ }^{20}$. La disparité des situations - en termes de temporalité, de situations personnelles, de résultats (le succès ou l'échec de la demande) - et la juxtaposition de leurs spécificités rendent possibles des interprétations valables à plus grande échelle. La densité des données ayant trait à chacune des situations, analysées par le recoupement des perspectives propres à plusieurs agents sociaux, rend compte des divers niveaux sur lesquels se joue l'intégration. Les données témoignent de la perspective des actrices et acteurs impliqués et rendent visibles leurs ressources et leurs capacités d'agir dans le contexte administratif et juridique étudié.

17. Bakhtine, 1987; Marková, 2007.

18. Berg, 2004.

19. Geertz, 1973, pp. 310-323.

20. Par exemple Fyljberg, 2011 ; Zittoun, 2017. 
Les analyses explorent différentes facettes de la négociation entre les agents de l'État et les personnes candidates quant à l'interprétation d'un cadre légal qui ne définit pas clairement l'intégration. Fondés sur l'analyse approfondie d'une série de cas particuliers dans un même contexte cantonal, les mécanismes mis en lumière ont une portée plus générale. Ils reflètent des processus auxquels sont soumis des requérantes et requérants dans d'autres cantons et ce qui se passe dans des procédures administratives portant sur d'autres démarches que celles liées à la naturalisation.

\subsection{LE TRAVAIL DE TERRAIN ET DE PRODUCTION DES DONNÉES}

Cette section présente dans un premier temps les spécificités et la pertinence du contexte choisi, celui du canton de Neuchâtel. Dans un deuxième temps, elle détaille le type de données utilisées et la manière dont elles ont pu être produites.

\subsubsection{UN TERRAIN ANCRÉ DANS LE CANTON DE NEUCHÂTEL}

La recherche s'est focalisée sur Neuchâtel en raison de son positionnement spécifique dans le domaine de l'intégration et de la naturalisation. Premier canton à avoir adopté, en 1996, une loi sur l'intégration ${ }^{21}$, Neuchâtel jouit d'une solide réputation de libéralisme en matière de naturalisation. Cette réputation est explicitement mise en lien avec l'existence d'une politique cantonale volontariste d'intégration, et étayée par un taux important, le deuxième de Suisse, d'octroi de la nationalité au regard de la population $^{22}$.

Ancré dans un environnement spécifique en termes politiques, sociaux et légaux, le travail de terrain s'est progressivement construit au fil des collaborations avec les différentes participantes et participants à la recherche. Par terrain, il ne faut pas entendre un lieu spécifique dans lequel les données seraient présentes indépendamment de la recherche, et en attente d'être récoltées. Ce qui est devenu le terrain n'existe pas comme préalable au déploiement de la recherche. Il a été façonné par l'objet de recherche, l'intégration, par les orientations respectives - l'intérêt porté à l'évolution de l'appareil législatif, au vécu des candidates et candidats, aux procédures administratives -, par le travail commun, ainsi que par les

21. Wichmann et al., 2011, p. 61.

22. Ibid., p. 35 . 
conditions rencontrées, la principale étant la réceptivité et la grande disponibilité des instances cantonales par rapport aux demandes de rendez-vous et de clarification des chercheuses. Le travail de terrain s'est ainsi négocié entre l'équipe de recherche et les différentes participantes et participants ${ }^{23}$, pour définir ensemble les rôles possibles des unes et des autres ${ }^{24}$. Les collaborations ont été nouées avec le personnel administratif, d'une part, et avec les candidates et candidats, d'autre part, ce qui a permis de faire émerger des données de manière processuelle. Les notes prises pendant les entretiens incluent des appréciations quant à l'atmosphère de la rencontre, notes qui «[mettent] en forme le discours produit dans la relation ternaire liant interviewé, interviewer et objet de l'entretien ${ }^{25}$.

\subsubsection{LES DONNÉES}

Les données sont le fruit de multiples rencontres: la rencontre des intérêts de recherche au sein de l'équipe et des méthodologies propres aux disciplines qui y sont représentées; la rencontre entre les savoirs académiques, les savoirs et expériences des candidates et candidats et les savoirs techniques et empiriques des employées et employés de l'État. Les données peuvent être regroupées en cinq types: les textes légaux et documents officiels; les données produites lors d'entretiens; les dossiers administratifs et personnels; les données résultant des observations et des vérifications faites tout au long de la recherche; et les données recueillies auprès des communes par questionnaire.

Le premier type est celui des textes légaux, établi sur la base d'une sélection des textes significatifs, constitué de lois fédérales et cantonales ainsi que d'autres textes s'y apparentant. Les deux lois fédérales qui mentionnent la nécessité d'une intégration - la loi sur les étrangers (LEtr) et la loi sur la nationalité (LN) - ont été lues à la lumière des changements qui les ont émaillées. La dimension diachronique apparaît dans la prise en considération des versions précédentes (à partir de 1848 pour la loi sur la nationalité, de 1931 pour la loi régissant le séjour et l'établissement des étrangers), ainsi que les changements introduits à partir de 2018, donc postérieurs au travail de terrain. La reconstruction diachronique inclut les messages du Conseil fédéral sur la

23. Berg, 2004.

24. O'Reilly, 2005; Telfer, 2004.

25. Levasseur et Haas, 2015, p. 2. 
question, qui permettent de retracer les enjeux successifs autour des notions d'incorporation, d'assimilation et d'intégration ${ }^{26}$. Elle comprend aussi la jurisprudence fédérale, lorsque celle-ci a contribué à dessiner les interprétations légitimes de la notion d'intégration.

Fixant le seuil minimum de conditions à remplir pour l'intégration, ces lois délèguent la mise en application aux cantons: les procédures impliquent la loi sur le droit de cité cantonal, la loi sur la cohésion multiculturelle, ainsi que la charte de la citoyenneté, document spécifique à Neuchâtel dont l'objectif est de présenter les fondements de la "république démocratique, laïque, sociale et garante des droits fondamentaux " ${ }^{27}$ (à savoir le canton) à toute personne nouvellement domiciliée sur le territoire cantonal. Il convient de considérer les modalités de répartition des compétences et des tâches entre les différentes instances, par exemple le mandat que le Service de la justice a confié au Service de la cohésion multiculturelle (COSM) consistant à mener une "enquête de naturalisation" auprès des candidates et candidats.

Les procédures ont été retracées en recoupant les informations de divers médias. Les sites web des instances concernées se sont révélés précieux, non seulement pour les informations qu'ils présentent, mais aussi par les vides qu'ils comportent, qui ont dû être comblés. La procédure exposée par les différentes employées et employés de l'État a été systématiquement confrontée avec les trajectoires qui ressortent des cas particuliers analysés. Cette mise en regard révèle le dynamisme des procédures, objet d'adaptations presque constantes - certaines en lien avec l'organisation des services, comme le remplacement de formulaires par une version actualisée; d'autres répondant à de nouveaux enjeux locaux, comme la délégation de l'enquête de naturalisation au COSM.

Les entretiens menés avec des candidates et candidats à la naturalisation ou à un changement de permis, et avec le personnel de l'État impliqué dans ces processus, constituent le deuxième type de données. Ces entretiens semi-dirigés et compréhensifs ${ }^{28}$ ont suivi un canevas général pour permettre d'aborder, sous forme de questions ouvertes, les thématiques générales au centre de la

26. Di Donato, 2016.

27. https://www.ne.ch/autorites/DEAS/COSM/conseil-information/PublishingImages/ Pages/Charte-de-la-citoyennet\%C3\%A9/mementoA4FRA.pdf (consulté le 29 janvier 2020).

28. Kaufmann, 1996. 
recherche. La grille a fonctionné comme un aide-mémoire plutôt que comme une succession de questions suivant un ordre strict. Les chercheuses menant les entretiens ont laissé les interlocutrices et interlocuteurs répondre de manière extensive aux questions, ne rebondissant sur des points qui paraissaient importants ou peu clairs que lorsqu'ils avaient terminé. Cela a favorisé les récits et les mises en forme personnelles. Tous les entretiens formels ont été enregistrés et ont fait l'objet d'une retranscription. Ces processus ont produit des données qualitatives qui ont ensuite été analysées plusieurs fois, selon différentes perspectives, de la manière la plus rigoureuse possible ${ }^{29}$; nous explicitons les méthodes d'analyse au début de chaque chapitre présentant nos analyses.

Les entretiens avec les candidates et candidats ont été réalisés dans des lieux choisis par les personnes elles-mêmes: dans leurs foyers respectifs, dans les locaux de l'université ou encore dans des cafés peu bruyants. Ces derniers ont été choisis par les interlocutrices et interlocuteurs parce qu'ils les fréquentaient comme clients ou, pour d'autres, parce qu'ils y travaillaient. En général, il y a eu un seul entretien par candidate ou candidat, mais certaines personnes ont été rencontrées à nouveau, soit pour compléter les informations, notamment par le biais des dossiers, soit parce que le premier entretien n'avait pu être enregistré, soit lorsque des candidates ou candidats voulaient faire part de l'avancement de leur demande.

Le travail de terrain a été mené selon les quatre modalités suivantes:

- l'ensemble de l'équipe de recherche a pris part à la première rencontre avec les représentantes et représentants de chacun des services concernés;

- Flora Di Donato et Anne Lavanchy ont réalisé les entretiens avec les candidates et candidats, en duo ou en solo selon les cas;

- Flora Di Donato, Élodie Garros et Anne Lavanchy ont travaillé directement avec les professionnelles et professionnels de la naturalisation;

- Anne Lavanchy a été chargée des contacts avec les communes, à savoir les entretiens avec deux Commissions des naturalisations et des agrégations, l'élaboration et l'envoi des questionnaires, puis le dépouillement des réponses. 
En incluant l'expérience subjective et les émotions des personnes impliquées dans les procédures et la manière dont elles interprètent le champ social, les entretiens fondés sur les procédures renseignent sur les rapports des candidates et candidats à la Suisse, mais aussi sur leurs rapports à la loi.

Les entretiens avec des représentantes et représentants de l'État visaient à saisir les manières d'agir et de se positionner par rapport à la procédure de naturalisation. La demande et question initiale était l'explication de la procédure, ce qui a permis ensuite d'entrer dans les détails des évaluations des documents et de l'intégration, de manière plus spécifique. Là encore, ce sont les logiques explicatives et interprétatives émiques qui ont été privilégiées, plutôt qu'une grille de questions fermées. Les agents de l'État ont été sollicités à plusieurs reprises afin de clarifier certains éléments. Quelques entretiens ont été menés de manière collective, par exemple celui avec la Commission chargée de l'examen préalable des demandes de naturalisation. Un entretien a été mené avec quatre personnes travaillant au Service des migrations, chargé de renouveler et d'accorder des permis de séjour, et deux entretiens formels avec les chefs de service et des collaboratrices et collaborateurs du Service de la cohésion multiculturelle (COSM) et du Service de la justice (JUST). Ces entretiens ont documenté les manières de travailler, le déroulement des procédures administratives, les enjeux posés par la formalisation de critères d'intégration et la prise de décision. Certaines personnes clés ont été rencontrées à plusieurs reprises, jusqu'à six fois, pour comprendre des cas particuliers, et des courriers électroniques ont été échangés pour éclaircir des doutes quant aux aspects techniques de leur travail.

Contrairement aux candidates et candidats, les fonctionnaires cantonaux n'ont pas pris part à la recherche pour des raisons liées à leur trajectoire biographique, mais en fonction de leur appartenance professionnelle et sur la base de l'accord de participation donné par les cheffes et chefs de services concernés. Il s'agit donc d'un public de participantes et participants captifs et non volontaires. La collaboration a été possible grâce à la volonté de transparence et à l'intérêt de leur part pour les demandes et analyses de l'équipe de recherche. Une journée de restitution et discussion avec les services cantonaux impliqués a été l'occasion de clarifier des éléments techniques, mais aussi de discuter des résultats, de la répartition des tâches entre les différents services, 
et d'esquisser les changements à venir, en lien avec la nouvelle loi fédérale sur la nationalité (LN) - entrée en vigueur entre-temps, le $1^{\text {er }}$ janvier 2018 - et l'actualisation qui en résulte de la loi cantonale sur le droit de cité neuchâtelois (LDCN).

Le troisième type de données est constitué par les dossiers personnels et administratifs des cinq personnes candidates qui, à la suite de l'entretien, ont accepté leur utilisation par l'équipe de recherche. L'expression "dossiers personnels» désigne tous les documents rassemblés et obtenus par ces personnes dans le cadre de leur demande. Y figurent les originaux ou copies des lettres envoyées, des documents officiels tels que les documents d'état civil, les lettres échangées avec les services de l'administration ou avec des avocats, les factures et émoluments payés. On y trouve aussi des documents liés à l'avancement de la procédure, détaillant les demandes d'informations complémentaires, les préavis et les décisions prises par les diverses autorités impliquées. Les «dossiers administratifs", archivés au Service de la justice, reprennent ces mêmes documents, mais dans un ordre variable, car ils sont réorganisés à plusieurs reprises au cours de la procédure. Par ailleurs, ils contiennent en sus certaines notes de service, courriers internes et correspondances entre des services administratifs. Les deux types de dossiers de ces cinq personnes ont été photocopiés et scannés, ce qui a rendu possible la reconstitution de cinq cas complets, préalable indispensable à l'analyse des trajectoires légale et personnelle.

Les observations forment le quatrième type de données. S’il ne s'agissait pas d'une observation participante au sens strict du terme, avec une immersion dans le quotidien des différents acteurs impliqués ${ }^{30}$, il a été possible de participer à divers moments significatifs du travail administratif et d'observer ces pratiques en passant quelques heures dans un service. Une des membres de l'équipe a assisté à une formation continue destinée au personnel portant sur les changements liés à l'introduction de la nouvelle loi sur la nationalité en 2018. Bien que ponctuels, ces moments permettent de documenter tout ce qui ne se dit pas, car trop trivial, allant de soi ou trop connu pour être encore verbalisé.

Dans la phase ultime de la recherche, toutes les communes neuchâteloises ont reçu un questionnaire élaboré à la suite

30. Lofland et al., 2006. 
des entretiens avec des élues et des élus de deux communes. Les réponses obtenues, le cinquième type de données, ont permis de comprendre le rôle des communes dans les deux phases de la procédure. Les rencontres avec des Commissions communales des naturalisations et des agrégations ont été utiles pour comprendre la dimension politique du traitement des demandes de naturalisation, et la partie technique de la procédure qui relève des communes. Il a été possible de participer à une séance de la Commission des naturalisations et des agrégations, émanant du Conseil général, l'organe législatif des communes à Neuchâtel. Un entretien (non enregistré) a été conduit avec la Chancelière et le responsable du Dicastère en charge de rédiger le préavis communal. Le responsable du Dicastère est membre du Conseil communal et représente donc le pouvoir exécutif, qui intervient pour donner un préavis. Il convient de préciser que les communes jouissent d'une grande marge de manœuvre quant à leur participation à la procédure et qu'elles gèrent selon diverses formules la question de la représentativité des partis politiques: certaines Commissions des naturalisations et des agrégations sont constituées d'un membre par parti représenté; d'autres prennent en considération l'importance des partis selon un système proportionnel. Les contacts avec les communes sont intervenus en fin de recherche, ce qui coïncidait avec le début de nouveaux mandats politiques suite aux élections de 2016: dans certains cas, les questionnaires ont été remplis par des membres qui n'avaient encore jamais eu l'occasion de siéger lors de séances de naturalisation (voir chapitre 5).

\subsection{LES CANDIDATES ET CANDIDATS}

La plupart des rencontres avec des personnes candidates ont été facilitées par des agents institutionnels. Le Service de la cohésion multiculturelle a donné l'impulsion à la recherche, puisque, à la suite d'une première prise de contact, l'un des employés a approché plusieurs personnes pour leur demander si elles acceptaient de communiquer leurs coordonnées. En second lieu, l'équipe a tenté de recourir à la méthode dite boule de neige en partant de ses propres réseaux ${ }^{31}$. Mais cette technique du bouche-à-oreille s'est révélée peu efficace; seule $M^{\text {me }}$ Line a été ainsi recrutée.

31. Dahinden et Effionay, 2009; Moret, 2017. 


\subsubsection{L'ANONYMISATION ET SES ENJEUX}

Ce sont donc, au total, dix candidates et candidats de nationalités variées, originaires de pays africains, du Moyen-Orient et d'Amérique latine, qui ont été interviewés. Leurs portraits individuels, rendus anonymes en termes de noms et de données biographiques, sont présentés ci-après. L'anonymisation est un processus nécessaire, mais délicat dans la mesure où il induit une tension entre la nécessité de transparence quant aux données et la protection de l'identité des participantes et participants. Il s'agit de pondérer entre ce qu'il est indispensable de dévoiler et ce qui ne l'est pas ${ }^{32}$. L'anonymisation est nécessaire à la mise à distance des données basées sur des cas particuliers, ce qui constitue une condition sine qua non du travail interprétatif qualitatif. Il passe par un travestissement des identités qui crée une altérité entre les acteurs réels et leurs personnages, et permet la généralisation analytique ${ }^{33}$.

En ce qui concerne les pseudonymes, le choix s'est porté sur des noms que l'on peut qualifier d'ici, et non pas sur des noms dont la consonance serait associée, par exemple, au pays d'origine ou à la langue maternelle de la personne concernée. Cette manière de faire est pertinente au regard de l'objectif de la recherche, qui n'était pas d'explorer des spécificités propres à certains types de trajectoires migratoires ou à des groupes de population spécifiques, mais de comprendre le positionnement des candidates et candidats par rapport aux procédures et leurs efforts pour façonner leur trajectoire en fonction des opportunités et des contraintes spécifiques à un moment et dans un lieu donnés ${ }^{34}$. Le choix de pseudonymes connotés comme locaux pour ces candidates et candidats dont le point commun est d'être ressortissants de pays tiers et soumis, à ce titre, à des contraintes légales spécifiques à ce statut permet d'éviter l'écueil du regard ethnicisant qui biaise nombre d'études portant sur la migration ${ }^{35}$.

Les spécificités des trajectoires biographiques ont été gommées, de même que les informations considérées comme annexes ou transposables d'une situation à une autre, tout en conservant les informations fondamentales. Ce processus relativise le côté

32. Saunders, Kitzinger et Kitzinger, 2015.

33. Flamant, 2005, p. 146.

34. Anderson et Calhoun, 1992. Notons cependant que le choix de ces pseudonymes «blanchit» nos interlocutrices et interlocuteurs, en rendant invisibles des caractéristiques (un prénom arabe ou peul par exemple) qui influencent le «feeling» des agents de l'État (Lavanchy, 2020).

35. Glick Schiller et Caglar, 2013. 
sensationnel des descriptions qui sont centrées sur l'objet de la recherche ${ }^{36}$. Les nationalités ne sont pas indiquées, mais les informations relatives à la raison de leur présence en Suisse (fuite en raison d'un régime dictatorial, d'une guerre, etc.) ont été conservées, car elles déterminent souvent le régime légal auquel ces personnes sont astreintes en Suisse. Dans les portraits qui suivent, le genre et l'âge (ou son estimation) ont été conservés, même s'ils n'ont pas été explicitement analysés. Les informations relatives aux types de permis de séjour ou d'établissement reflètent la situation réelle des candidates et candidats. Y figurent le type de permis détenu au moment de la demande, ainsi que l'éventuelle succession de permis au cours du parcours de vie et les modalités d'entrée en Suisse (par migration ou par naissance).

\subsubsection{PORTRAITS}

Nous présentons ci-après les portraits des personnes candidates dont nous avons recueilli le témoignage, et qui ont accepté de mettre leurs dossiers à notre disposition. Ces situations ne sont pas représentatives, en termes numériques, de la majorité des dossiers traités par l'administration. Le premier tri effectué par le Service de la cohésion multiculturelle ciblait explicitement des cas intéressants, car problématiques, selon l'interprétation qui a été faite de notre demande. Dans un second temps, des dossiers considérés comme plus standards par le Service de la justice ont été inclus pour pondérer cette dimension qualifiée de problématique, avec notamment un cas de naturalisation ordinaire par procédure simplifiée. La recherche initiale d'une parité entre hommes et femmes ne se donne pas à voir dans les résultats finaux, puisqu'une seule femme a transmis l'ensemble de son dossier, contre quatre hommes.

Il aurait été difficile de constituer un échantillon représentatif, car il n'existe aucune donnée statistique, aux niveaux fédéral et cantonal, sur le nombre de demandes déposées. Les chiffres mentionnés servent d'indicateurs et ont été extrapolés des rapports d'activité des institutions avec lesquelles l'équipe de recherche a collaboré.

Présentés dans l'ordre alphabétique, les portraits sont introduits par un titre indiquant le pseudonyme, le type de procédure engagé et son résultat, et si nous disposons d'un dossier complet ou non. 
Ce titre descriptif est suivi par une expression extraite des entretiens, synthétisant la manière dont chaque candidate ou candidat a vécu la procédure. Les citations tirées des entretiens entre chercheuses et personnes candidates sont signalées par des guillemets. Par ailleurs, dans l'ensemble de l'ouvrage, les citations utilisées tirées des entretiens ont été mises en forme de manière à les rendre plus lisibles.

\section{Armand}

Révocation de la naturalisation facilitée:

\section{"C'était un cauchemar"}

M. Armand est âgé d'une quarantaine d'années. Il est propriétaire d'un restaurant qui accueille aussi des événements culturels. Arrivé en Suisse en raison de la guerre dans son pays, il requiert l'asile et obtient le statut de réfugié. Après de nombreux déménagements, M. Armand rencontre une femme qui lui donne des cours de français; ils tombent amoureux et se marient. Conseillé par une avocate, il fait les démarches pour obtenir un permis $\mathrm{B}$, puis dépose une demande de naturalisation facilitée, qu'il obtient en un an et demi, après cinq ans de séjour en Suisse. Après huit ans de vie commune avec sa femme, il la quitte pour une autre, pour laquelle il dit avoir eu un "coup de foudre». L'annonce officielle de séparation au contrôle des habitants déclenche une procédure du Secrétariat d'État aux migrations (SEM), visant, selon ses termes, à lui « retirer la nationalité», au motif que, au moment des démarches, les conditions de son obtention n'étaient plus remplies, le couple n'en étant plus un. La procédure est éprouvante, M. Armand et son avocat portent l'affaire jusqu'au Tribunal fédéral, où il obtient finalement gain de cause. Pour M. Armand, le soutien de son épouse légitime, auprès de laquelle il était retourné entre-temps, a été capital dans son combat contre la menace de retrait de naturalisation, qu'il qualifie de cauchemar. Dans son récit, l'administration apparaît comme une instance opaque et incompréhensible, et le respect des lois comme aléatoire.

\section{Bruno}

\section{Un permis menacé: "Une vie empêchée"}

M. Bruno est arrivé en Suisse à la suite de la guerre dans son pays dans les années 1980. Alors âgé de 19 ans, il obtient par sa famille, bien placée, un emploi dans le corps diplomatique à l'ONU. Après huit ans, la situation politique étant toujours tendue dans son pays, 
il obtient un statut d'admission provisoire en Suisse. Contrairement à ses attentes, ce permis ne contribue pas à stabiliser sa situation précaire. Il est renouvelé pendant neuf ans, puis $\mathrm{M}$. Bruno obtient un permis B. Il souhaite ensuite obtenir un permis $\mathrm{C}$, mais sa demande est rejetée, car il est au chômage; il en va de même pour une demande de naturalisation. Il est maintenant en Suisse depuis un peu plus de trente ans, âgé d'une cinquantaine d'années, au bénéfice de l'aide sociale, et menacé de renvoi. Globalement, M. Bruno porte un regard amer sur sa trajectoire: il a l'impression d'avoir fait une erreur initiale lors de sa demande d'asile, le permis F se révélant un "permis foutu", qui marque sa trajectoire de manière indélébile et qui l'a privé de la vie à laquelle il aurait pu aspirer.

\section{Charles}

Rejet de la demande de naturalisation ordinaire (dossier complet): "Un processus de désintégration"

Le dossier de naturalisation ordinaire déposé par M. Charles a ouvert une procédure de plus de huit ans, sans aboutir. Le Conseil communal a émis un préavis négatif, qui sera confirmé une seconde fois. Ces deux préavis bloquent la situation et empêchent $M$. Charles d'obtenir l'autorisation fédérale de naturalisation, préalable indispensable à l'obtention du droit de cité neuchâtelois. Le cas de M. Charles, sur lequel reviennent les chapitres 5, 6, 7 et 8, illustre l'opacité de la procédure, la difficulté pour le candidat de la comprendre, ainsi que l'importance de la temporalité de certaines actions: lassé, $\mathrm{M}$. Charles abandonne la procédure et renonce à la naturalisation, se sentant pris dans un processus de "désintégration" plutôt que d'intégration.

\section{$\mathrm{M}^{\mathrm{me}}$ Dominique}

Demande de renouvellement de permis B:

"C'est vraiment difficile, difficile"

À l'aube de la quarantaine, $\mathrm{M}^{\mathrm{me}}$ Dominique est arrivée en Suisse comme requérante d'asile, et a obtenu le statut de réfugiée. Elle a épousé un réfugié, avec lequel elle a quatre enfants, le dernier étant né Suisse, car son père a été naturalisé entre-temps. Son mari absent depuis plusieurs mois, elle se retrouve seule à élever ses enfants et dépend de l'aide sociale. Elle craint que son permis B ne soit pas renouvelé et mentionne les lettres "de menace de renvoi» 
envoyées par l'administration. L'entretien, mené à son domicile, ne permet cependant pas de comprendre sa situation administrative, qui reste confuse, tout comme les raisons et la signification de la longue absence de son mari. Il est marqué par des difficultés de communication entre les chercheuses et $\mathrm{M}^{\mathrm{me}}$ Dominique, qui maîtrise relativement mal le français.

\section{$\mathrm{M}^{\mathrm{me}}$ Elvire}

\section{Demande de naturalisation en cours: "Je suis en chemin"}

$\mathrm{M}^{\mathrm{me}}$ Elvire reçoit les chercheuses chez elle, avec sa fille majeure. Elle travaille comme employée domestique, mais a dû subir une importante opération des jambes. Elle est en convalescence, ce qui lui pèse, car elle ne sait pas si elle pourra retourner chez ses employeurs. Alors jeune adulte, elle a fui son pays il y a une quinzaine d'années, en raison d'une histoire familiale violente marquée par le crime organisé et des abus. Le voyage l'a menée en Espagne, puis dans un centre au Tessin, et elle a appris le français dans un centre pour requérants en Valais. Elle a obtenu un permis $\mathrm{N}$, de requérante d'asile, puis un permis $B$ et enfin son permis $C$ actuel. Elle a rencontré son mari, dont elle a divorcé il y a un an, en Suisse. L'avocat qui l'a accompagnée pour son divorce s'est battu pour son droit de rester en Suisse et l'a encouragée à demander la nationalité suisse pour ses enfants. À la suite de cela, elle a entamé une procédure de naturalisation pour elle et ses deux enfants encore mineurs. En l'absence de son dossier, il est difficile de comprendre sa situation, et notamment le lien qu'elle fait entre son permis $\mathrm{C}$ et son mariage, et la menace qui pèserait sur le renouvellement de son permis en raison de son divorce.

\section{François}

Demande de naturalisation refusée (dossier complet): "Moi tout seul, ça, c'est la vie, c'est dur dur"

M. François a 60 ans. En 1999, il a déposé une demande d'asile en Suisse, après avoir quitté son pays en guerre, où il a été victime d'une violente agression qui lui laisse des séquelles importantes. Sa femme et ses six enfants étant restés dans son pays, la solitude lui pèse. Sa demande d'asile est refusée, mais il obtient une admission provisoire (permis F) en 2001, puis un permis B quelques années plus tard. En 2011, il dépose une demande de naturalisation. L'enquête de naturalisation faite par le Service de 
la cohésion multiculturelle indique que sa maîtrise du français n'est pas suffisante et, en 2013, le Conseil communal donne un préavis négatif pour cette raison. L'avocat sollicité par M. François informe l'administration du maintien de la demande en soulignant que son niveau de français provient du handicap généré par l'agression dans son pays. Une expertise médicale confirme des difficultés de langage et d'apprentissage liées au traumatisme subi. En décembre 2013, il reçoit l'autorisation fédérale. Début 2014, il demande à la commune l'octroi du droit de cité. Cette dernière s'informe auprès du canton et accorde le droit de cité communal en août 2014. M. François est naturalisé en mars 2015.

\section{Gilbert}

Refus de prolongation du permis étudiant: "La violence de l'administration"

M. Gilbert est venu en Suisse avec un visa d'étudiant. Il entreprend un bachelor d'abord à Genève, puis à Neuchâtel, et est confronté à des difficultés administratives pour poursuivre un master dans un autre canton; il ne peut pas déménager, car cela impliquerait de recommencer les procédures. Après un échec aux examens, il change de filière d'études, dépasse le nombre d'années autorisé pour un bachelor et est menacé de renvoi. Pendant ce temps, il a rencontré une femme avec un passeport européen, qu'il épouse en Suisse. Apparemment conseillé par un avocat, le couple utilise la possibilité de libre circulation de son épouse pour qu'elle puisse le rejoindre; ils ont un enfant né en Suisse. Il peut rester en Suisse grâce au regroupement familial avec une ressortissante de l'espace Schengen, mais il ressent sa situation comme précaire, et considère que l'administration fait violence aux étudiantes et étudiants de pays tiers.

\section{Johan}

\section{Naturalisation ordinaire selon la procédure simplifiée} (dossier complet) : "Je me suis fait Suisse"

Né en Suisse, détenteur de deux passeports de pays européens, M. Johan a déposé à l'âge de 37 ans sa demande de naturalisation en même temps que celles de sa compagne (européenne) et de leur fille - n'étant pas mariés, ils ne peuvent faire une seule demande. La requête suit la procédure simplifiée, qui comprend une enquête de naturalisation du Service de la cohésion multiculturelle, sans que ce dernier ne rencontre M. Johan. L'autorisation fédérale de 
naturalisation est accordée suite au préavis positif du Conseil communal, et $\mathrm{M}$. Johan et sa fille obtiennent la nationalité suisse. Lors de l'entretien de recherche, il ressort que M. Johan n'est pas du tout informé des différents acteurs institutionnels intervenant dans le processus, il ne garde en mémoire que le rôle de la commune, et non celui du canton.

\section{Kirin}

Naturalisation ordinaire en attente (dossier complet): "Le trou, il est difficile à remonter"

M. Kirin a 45 ans. Il est arrivé en Suisse à l'âge de 19 ans, en 1981, fuyant la dictature dans son pays. Il dépose d'abord un dossier de demande d'asile à Genève, puis s'installe dans le canton de Neuchâtel, où il obtient un permis d'établissement (permis C) en 1984. Il se marie en 2003 avec une femme également étrangère, dont il divorce sept ans plus tard. En 2004, il mandate un avocat pour initier la procédure de naturalisation. En 2008, le département demande une enquête approfondie à la police cantonale. Près de neuf mois plus tard, le rapport de cette dernière fait état d'un «lourd passé judiciaire» et du "non-paiement de contributions». Le Conseil communal donne un préavis négatif. Le dossier est envoyé à l'Office fédéral des migrations (ODM) pour obtenir l'autorisation fédérale, qui, dix-huit mois plus tard, demande des précisions. M. Kirin fournit à l'ODM plusieurs documents. En février 2013, l'ODM lui adresse un courrier pour l'informer de l'octroi de l'autorisation fédérale de naturalisation et du préavis négatif de la part des autorités cantonales, précisant qu' "une naturalisation sur le plan cantonal et communal paraît dès lors hypothétique». M. Kirin maintient sa requête. Le Conseil communal octroie le droit de cité en avril, mais, en décembre 2013, la Commission chargée de l'examen préalable des dossiers de naturalisation au niveau cantonal met en suspens le dossier en raison d'impayés.

\section{$\mathrm{M}^{\mathrm{me}}$ Line}

Demande de naturalisation ordinaire acceptée (dossier complet): "Pour que la Suisse me reconnaisse comme je suis" $\mathrm{M}^{\mathrm{me}}$ Line est arrivée en Suisse jeune, avec ses parents, qui y déposent une demande d'asile. La famille obtient le statut de réfugié. $\mathrm{M}^{\mathrm{me}}$ Line effectue sa scolarité en Suisse, y rencontre son mari, étranger d'un pays tiers, et fonde une famille. Elle dépose une demande de 
naturalisation qui va suivre un périple de dix-huit ans. À la suite de deux refus pour des raisons différentes (manque d'intégration, puis impôts non payés), $\mathrm{M}^{\mathrm{me}}$ Line s'engage dans un combat de plusieurs années et saisit la justice à plusieurs reprises. Différences instances judiciaires - en dernier lieu le Tribunal fédéral, saisi d'un recours constitutionnel subsidiaire - reconnaissent que la procédure de naturalisation est "viciée» et soulignent les lacunes dans l'instruction du dossier. En août 2016, après dix-huit ans de procédure, $\mathrm{M}^{\mathrm{me}}$ Line obtient la nationalité suisse.

\subsection{LES MEMBRES DES ADMINISTRATIONS}

Les employées et employés de l'État qui ont participé aux entretiens sont des membres du Service de la cohésion multiculturelle, du Service de la justice et, initialement, du Service des migrations. Ayant recentré la recherche sur la procédure de naturalisation, ce dernier n'était plus concerné en premier lieu, car il intervient pour délivrer des permis de séjour et d'établissement. Les pseudonymes rendent compte des appartenances institutionnelles, les deux premières lettres du nom renvoyant aux initiales du service correspondant. Ainsi $\mathrm{M}^{\mathrm{me}}$ Michaux est une personne du Service des migrations, MM. Corentin, Costin et Coronte travaillent au Service de la cohésion multiculturelle, et $\mathrm{M}^{\text {mes }}$ Juliette et Justine et MM. Jules et Julien au Service de la justice. Il n'y a dans ces cas pas de correspondance de genre entre les pseudonymes et les personnes réelles. Les mêmes règles président à l'établissement de pseudonymes pour les membres des Commissions communales des naturalisations et des agrégations, dont les noms choisis commencent par la lettre «n» (naturalisation): MM. Nasser, Noël et Norman, $\mathrm{M}^{\text {mes }}$ Nadège, Natacha et Norma.

Précisons que les histoires de demande de naturalisation présentées dans ce livre se déroulent dans le temps et à des périodes différentes. Méthodologiquement, cela incite à une grande prudence au regard de leur contextualisation, et ce à deux niveaux. Premièrement, la loi sur la nationalité a connu des modifications, entraînant des changements dans les conditions matérielles définies pour mesurer l'intégration et, de ce fait, dans la procédure de naturalisation. Adoptées par l'Assemblée fédérale en juin 2014, les modifications liées à la révision totale de la loi sur la naturalisation sont entrées en vigueur au $1^{\text {er }}$ janvier 2018, période postérieure au travail de 
terrain. Deuxièmement, la dimension temporelle des demandes de naturalisation nécessite une précaution terminologique: les analyses reprennent les appellations présentes dans les courriers qui proviennent de différentes autorités, communales, cantonales et fédérales. Or, beaucoup de ces appellations ont été modifiées, et ce à plusieurs reprises, par exemple lors de la suppression d'un service ou la réorganisation d'un département. C'est pourquoi il est utile de présenter brièvement les deux services cantonaux centraux dans cette recherche, de même que la Commission chargée de l'examen préalable des demandes de naturalisation et, enfin, au niveau fédéral, le Secrétariat d'État aux migrations.

$\mathrm{Au}$ moment de notre recherche, le Service de la justice a la responsabilité de traiter les demandes de naturalisation au niveau cantonal par le biais d'un secteur spécifique, nommé secteur naturalisation. Ce dernier a été créé en septembre 2014, à la suite de la réorganisation du Service de la justice (arrêté du 20 novembre 2014), qui voit l'ancien office de la population réparti en différents secteurs. Le Service de la justice dépend du Département de la justice, de la sécurité et de la culture, qui a connu des géométries variables. Avant août 2013, il comprenait la justice, la sécurité et les finances (sous l'acronyme DJSF), qui avait lui-même succédé en 2005 au Département de la justice, de la santé et de la sécurité. Le texte privilégie l'emploi de l'expression "Service de la justice» (ou parfois son acronyme, JUST, qui est couramment utilisé par les personnes des services cantonaux), même si la majeure partie du terrain s'est déroulée en collaboration avec le secteur naturalisation.

Le Service de la cohésion multiculturelle représente et ancre institutionnellement la réputation neuchâteloise de tolérance. Connu sous cette appellation depuis 2009, le service est l'héritier du Bureau du délégué aux étrangers (BDE) créé en 1990. En 1996, dans le cadre de la politique publique d'intégration des étrangers, le Grand Conseil neuchâtelois adopte la première loi sur l'intégration des étrangers en Suisse. Elle fournit une base juridique aux activités du Bureau du délégué aux étrangers et lui confere comme principales missions de "favoriser des relations harmonieuses entre Suisses et étrangers", de "promouvoir l'égalité des droits et des devoirs pour tous les habitants du canton" et de "développer les mesures d'intégration des étrangers ${ }^{37}$. En 2007, le Conseil d'État décide

37. Rapport de gestion du Département de l'économie 2008, http://www.ne.ch/autorites/ CE/RappGestion/2008/DEC_2008_complet.pdf. 
de transférer les enquêtes de naturalisation ordinaire de la police cantonale au BDE. Le transfert devient effectif en janvier $2010^{38}$ et, cinq ans plus tard, les enquêtes d'intégration sont imputées au secteur naturalisation, né de la réorganisation du service.

La Constitution de la République et Canton de Neuchâtel en vigueur dès le $1^{\text {er }}$ janvier 2002 a transféré la décision cantonale d'octroi des naturalisations du Grand Conseil au Conseil d'État. Pour répondre aux exigences de cette nouvelle compétence, le Conseil d'État a créé, le 20 février 2002, une Commission chargée de l'examen préalable des dossiers de naturalisations. Cette commission a pour mission d'examiner "tous les dossiers de naturalisation qui peuvent faire l'objet d'une décision et [d'adresser] un rapport au Conseil d'État avec ses propositions" (arrêté du 20 février 2002). La commission est composée de quatre membres: le chef du Service de la justice, la responsable du COSM (à l'époque BDE), le chef du Service des migrations (à l'époque Service des étrangers), ainsi qu'une personne du secteur naturalisation du Service de la justice (JUST-Naturalisation) qui assure le secrétariat général. Les membres de cette commission livrent un préavis pour chaque dossier. Un rapport, rédigé par le secrétaire de la commission et signé par le chef du Service de la justice, consigne les décisions. Une fois signé, le rapport est envoyé au Conseil d'État, qui prend la décision cantonale et envoie le dossier au SEM.

Le Secrétariat d'État aux migrations (SEM), qui relève du Département fédéral de justice et police (DFJP), a pris la place de l'Office fédéral des migrations (ODM) le $1^{\text {er }}$ janvier 2015, en raison de l'importance grandissante de la thématique des migrations et d'un champ d'activité qui s'est complexifié. L'ODM était né le $1^{\text {er }}$ janvier 2005 de la fusion de l'Office fédéral des réfugiés (ODR) et de l'Office fédéral de l'immigration, de l'intégration et de l'émigration (IMES). Le SEM est l'organe compétent pour traiter les affaires de naturalisation au niveau fédéral, par le biais de sa division nationalité. Dans la pratique, seuls les dossiers des requérantes ou des requérants qui sont aptes à la naturalisation (selon les critères d'intégration définis selon l'art. $14 \mathrm{LN}$ ) sont envoyés au niveau fédéral, la responsabilité de l'examen formel et matériel des demandes étant du ressort des communes. Il convient

38. Rapport de gestion du Département de l'économie de 2010, http://www.ne.ch/ autorites/CE/RappGestion/2010/Rap_gestion_DEC_2010.pdf. 
de préciser que l'ouvrage cite, en principe, les institutions existant au moment où les dossiers analysés ont été traités.

\subsection{DES DONNÉES EMPIRIQUES AUX CAS}

Plusieurs étapes ont été nécessaires pour constituer les cas à partir de ces données, et offrir des analyses sur les différents points traités. Dans un premier temps, le travail a porté sur les documents matériels (dossiers administratifs et personnels), qui vont d'une vingtaine de pages à plus de soixante. L'objectif était de reconstruire de manière chronologique la trajectoire administrative de chaque demande. La reconstitution s'est révélée beaucoup plus ardue qu'un simple classement des informations, en raison de la simultanéité de certains actes administratifs et du fait que les informations quant aux procédures, basées sur le cadre légal, les sites web et les entretiens préliminaires avec les représentantes et représentants des institutions, n'ont pas permis de mettre en ordre les différentes étapes (cf. chapitre 5). La reconstitution s'est initialement fondée sur le cas éminemment complexe de $\mathrm{M}$. Charles, qui suit une procédure légèrement différente de celle qui était en vigueur lors de la recherche. Mais ce travail, avec les nombreuses vérifications et explications qu'il a nécessitées, s'est révélé très utile pour mettre au jour certaines ambiguïtés de la procédure et ses zones d'ombre.

La deuxième étape a été de mettre cette reconstitution en regard avec les données tirées des entretiens avec les candidates et candidats: représentations de la trajectoire administrative, éléments biographiques en rapport avec la procédure, interprétations des décisions, des demandes de compléments ou d'autres courriers de l'administration. Ce parallèle a fait émerger des constructions de sens de l'expérience administrative. Il a montré comment certaines compréhensions des candidates et candidats s'écartent de la conception administrative, comme lorsqu'il met en évidence que M. Charles, qui considérait avoir essuyé deux refus de naturalisation, n’avait en réalité entamé qu'une seule procédure, restée sans décision.

Ces deux étapes ont nécessité d'imprimer les dossiers, de comparer pièce par pièce les documents, et de synthétiser les différences. Il a ensuite été possible de construire le déroulement chronologique de chacun de ces parcours. Au final, à chaque personne candidate correspondent trois dossiers distincts: le dossier personnel, avec des documents dont l'ordre nous a paru parfois aléatoire, parfois 
relativement chronologique; le dossier administratif, avec des documents classés par le Service de la justice; et le dossier que nous avons reconstitué à partir de ces deux sources dépecées, dont les pièces ont été ordonnées de manière chronologique autant que faire se peut, puisque certains courriers se chevauchent, certaines demandes sont différées, et certaines réponses sont tardives.

Confronter les dossiers et les entretiens biographiques a documenté les différentes logiques à l'œuvre dans l'intégration: éclairer les spécificités de chacune de ces logiques illustre les négociations de sens et la manière dont leurs mises en forme sont légitimées. Les difficultés rencontrées ont mis en évidence à la fois l'opacité du travail administratif et la nécessité, pour tous les acteurs du champ de l'intégration officielle, de créer du sens à partir de leurs positionnements respectifs. En aucun cas l'analyse n'avait pour objectif de déterminer quelle serait la version la plus correcte.

Si ces deux premières étapes ont été en bonne partie le fruit d'un travail collectif, la suite du travail interprétatif s'est déroulée de manière plus autonome, parfois individuellement, parfois à deux, en croisant les perspectives, en fonction des intérêts, des disciplines et des méthodes respectives. Les différents chapitres analytiques précisent la démarche choisie, abductive ou réitérative, dans les analyses psychologiques et sociolégales de l'agentivité (chapitres 6 et 7); inductive dans l'analyse des prises de décision institutionnelles (chapitre 8).

\subsection{ENJEUX ÉTHIQUES: LES IMPACTS DE LA RECHERCHE}

Pour compléter ce chapitre, il convient de préciser trois types de questions éthiques soulevées par la recherche: le premier en termes de confidentialité; le deuxième lié aux attentes réelles ou potentielles des différentes participantes et participants; le dernier quant aux positionnements de l'équipe de recherche.

\subsubsection{LES DONNÉES ET LEUR PROTECTION}

Le principal enjeu éthique de la recherche concerne les questions de la confidentialité et du potentiel impact pour les différentes participantes et participants. En ce qui concerne la protection des données, nous avons garanti leur anonymisation et la confidentialité des entretiens. Un document élaboré en collaboration avec le Préposé intercantonal à la protection des données a été soumis aux candidates et candidats, qui récapitulait ces points et stipulait 
qu'elles et ils pouvaient revenir sur leur participation en tout temps au cours de l'entretien. Le consentement a été donné par signature. Un autre document présentait la requête de consultation des dossiers personnels et administratifs, qui autorisait les instances cantonales à permettre la photocopie, à des fins d'analyse, des documents archivés dans les administrations.

La nécessité de travailler sur les dossiers administratifs a donc impliqué une levée partielle de l'anonymat: les services concernés ont inévitablement su avec qui l'équipe avait travaillé. C'est pourquoi le choix s'est porté sur des cas qui étaient clos, puisque l'étude ne cherchait pas à influencer le traitement administratif ni à soutenir la cause individuelle de l'une ou l'autre des personnes interrogées. Pourtant, dans un cas spécifique, la procédure qui avait abouti à un refus initial a été poursuivie et s'est achevée, au cours de la recherche, par un octroi. La personne concernée a considéré que sa participation à la recherche avait eu un impact positif, en accélérant la procédure et en incitant les institutions à trouver une solution à une situation qui s'était apparemment enlisée. Par ailleurs, nous ne pouvons exclure qu'en cas de dépôt d'une nouvelle demande par une personne ayant participé à la recherche, nos analyses puissent influencer le traitement administratif, étant donnée la concentration des compétences institutionnelles entre les mains d'un nombre restreint de personnes, et le fait que des agents de l'État peuvent avoir une longue pratique professionnelle.

La question de la confidentialité touche aussi les professionnelles et professionnels qui travaillent pour le canton. En vue d'assurer un maximum de transparence, l'équipe a discuté avec eux à plusieurs reprises du fait que, dans un contexte aussi restreint, il peut se révéler aisé, pour des personnes candidates, de savoir qui sont les agents de l'État impliqués, ou du moins de faire des suppositions quant à leur identité. De manière similaire, l'anonymat n'a pas pu être totalement garanti entre collègues de différents services, ou entre des membres de commissions communales et des fonctionnaires cantonaux, car il est probable que des profils soient identifiables pour qui est familier des services.

\subsubsection{LES ATTENTES}

Lors du premier contact avec les personnes candidates, notre description de la recherche insistait sur le fait qu'il ne s'agissait pas d'aider à résoudre leur situation spécifique, en les accompagnant 
par exemple dans le cadre d'un recours, mais de comprendre leurs vécus et actions pour rendre public ce qu'elles vivent. Les analyses seraient potentiellement bénéfiques pour des personnes qui allaient initier ces démarches, et pourraient faire réfléchir les agents de l'État sur leurs pratiques administratives et même, éventuellement, les amener à les modifier. Malgré ces précautions, nous avons été confrontées à des attentes explicites de certaines personnes candidates quant à l'aide concrète que nous pourrions apporter, notamment en tant que juristes et dans le cas de procédures s'étendant sur des temporalités longues. D'autres ont adopté des postures plutôt défensives, en revenant sur leur décision de transmettre leurs dossiers, ou en ne donnant pas suite à nos relances pour obtenir les documents pourtant promis.

\subsubsection{NOS POSITIONNEMENTS}

Présenter la recherche comme pouvant, potentiellement, influencer les procédures et manières de faire des agents de l'État a positionné d'emblée l'équipe dans un champ social fait de tensions et d'antagonismes. En effet, la plupart des dossiers rassemblés sont considérés comme "problématiques" par les agents de l'État. Du point de vue des résultats, ces dossiers ne représentent pas de manière équilibrée le travail fait dans les administrations cantonales: en majorité, les dossiers se terminent par des refus à l'une ou l'autre étape, ce qui n'éclaire qu'une réalité partielle des procédures de naturalisation. Pour atténuer les effets de ce biais méthodologique, pour objectiver la recherche et les conditions de production de la connaissance qui en est tirée ${ }^{39}$, il convient donc d'expliciter nos positionnements et leur origine.

Le décentrement réflexif clarifie les conditions de reconstruction des $\operatorname{cas}^{40}$ : inscrire la recherche dans un objectif d'amélioration a conduit, et même légitimé, la surreprésentation des cas problématiques. Les relations de recherche avec des membres perçus comme appartenant à une certaine élite, ou à des groupes sociaux dominants, investis d'un pouvoir statutaire, peuvent amener les chercheuses à des attitudes de précaution, de distance et de souci d'objectivité. Les relations nouées avec des personnes considérées

39. Leservoisier, 2005, p. 22 ; voir aussi Fassin, 2008, p. 9, ainsi que Althabe et Hernandez, 2004.

40. Comaroff et Comaroff, 2003; Fassin, 2008; Nader, 1997; Olivier de Sardan, 2000; Lavanchy, 2013; Boden, Epstein et Latimer, 2009. 
comme n'appartenant pas à ces groupes peuvent quant à elles mettre en scène empathie, souci d'égalitarisme, de réciprocité et d'authenticité ${ }^{41}$.

Bien qu'il ait été possible de complexifier ce point de vue en développant la collaboration avec les agents de l'État, il est resté visible dans les stratégies relationnelles avec les différents groupes sociaux inclus dans la recherche. Les vécus respectifs des membres de l'équipe ont été explicitement mobilisés à différents moments de la recherche, pour revendiquer certaines places et statuts qui ont servi la compréhension située de ce champ de tensions ${ }^{42}$. Ainsi, les chercheuses ayant mené les entretiens avec des candidates et candidats ont parfois recouru aux spécificités de leur propre statut Suissesse/étrangère), se référant à des expériences comparables à celles des personnes interviewées. Elles ont opté pour une position neutre face à certaines candidates et candidats, alors qu'à d'autres moments une relation plus empathique, misant sur la connivence, s'est mise en place. Ces stratégies plus ou moins explicites et conscientes ont dévoilé des jeux entre des positions d'insider ou d'outsider par rapport à la question de l'intégration. Le fait d'occuper de telles positions, ou plus précisément de les rendre visibles à certains moments, indique bien que la fonction de chercheuse ou de chercheur n'efface pas la pluralité des identités sociales de ceux-ci, ni ne gomme leur implication dans ce qu'Elias nomme les affaires sociales et politiques de leur époque. Le fait que ces affaires les concernent directement ${ }^{43}$ conditionne leur intelligence par rapport aux problèmes analysés ${ }^{44}$. En d'autres termes, nos trajectoires personnelles et professionnelles nous amènent à développer un regard spécifique sur le choix et l'objet de notre recherche, elles outillent notre sensibilité et nos capacités d'analyse.

Au cours de la recherche, divers éléments ont enrichi les réflexions au sujet de nos positionnements. D’une part, travailler en équipe a complexifié ces équations personnelles ${ }^{45}$ et les réflexions sur le rapport engagé avec les participantes et participants ${ }^{46}$. Au sein de l'équipe ont surgi des lectures diverses, parfois antagonistes, de ce qui s'est joué dans les relations de terrain. L'équipe a été traversée

41. Blee, 1993 , p. 356.

42. Citons par exemple les travaux de Favret-Saada, 1977, et Bourdieu et Wacquant, 1992.

43. Elias, 1993.

44. Ibid., p. 29.

45. Leservoisier, 2005.

46. Weller, 2009, p. 322. 
de dynamiques d'identification à géométrie variable. Selon les moments et les enjeux, des tensions qui ont surgi, certains positionnements face à l'objet "intégration » et face aux institutions qui la fabriquent ont été revendiqués ou attribués.

D'autre part, les lectures de ces relations et plus spécifiquement des intentions prêtées aux interlocutrices et interlocuteurs variaient en fonction de l'implication, directe ou non, dans la relation de recherche. Mener un entretien ou le découvrir à travers une transcription n'amène pas toujours les mêmes compréhensions de certaines interactions. Les expériences directes colorent - à tort ou à raison - les lectures des transcriptions. La connaissance directe ou indirecte génère parfois des interprétations opposées quant aux attitudes des agents de l'État. Cependant, même lorsque l'ensemble de l'équipe a pris part à certaines rencontres, les compréhensions pouvaient diverger.

\subsection{SYNTHÈSE: UN TERRAIN CONSTRUIT EN COLLABORATION}

Fondée sur des textes légaux, sur des données qualitatives ${ }^{47}$ combinant des approches inductive, perspectivale, pragmatiste et dialogique, la méthodologie que nous avons adoptée prend en considération tant nos ancrages spécifiques disciplinaires que les positionnements des différentes actrices et acteurs sociaux participant à la recherche. La démarche choisie considère que nous faisons partie intégrante du champ exploré, avec nos positionnements personnels, épistémologiques et disciplinaires. La confrontation et le progressif tissage entre ces spécificités de l'équipe de recherche ont permis d'approfondir notre perspective réflexive, en intégrant les différentes sensibilités qui enrichissent la réflexion. Élaborer conjointement et à l'intersection de nos disciplines respectives les multiples critères nécessaires à la production du matériel empirique représente un défi de taille. Le relever nous a menées à dépasser nos limites individuelles et disciplinaires sur ce que sont les données pertinentes, le degré d'anonymisation, la manière de présenter les différentes personnes participant à la recherche, la façon de les recruter et d'inclure des actrices et acteurs d'abord périphériques, comme le personnel administratif ou les élues et élus politiques communaux. Sur cette base commune, le travail interprétatif s'est 
fait de deux manières: certains chapitres suivent des méthodes plus spécifiquement ancrées dans nos domaines respectifs, comme les chapitres 3 et 4, qui sont d'orientation juridique, le chapitre 6 , qui relève de la psychologie socioculturelle. D'autres ont été élaborés de manière collaborative, combinant le droit et l'anthropologie des institutions (chapitre 5), l'approche socioclinique du droit et la psychologie sociale (chapitre 7), ainsi que la psychologie sociale et l'anthropologie (chapitre 8). 


\section{PARTIE II CONSTRUCTION DU CONTEXTE INSTITUTIONNEL}





\title{
3. RETRACER LA NOTION D'INTÉGRATION: HISTOIRE DU DROIT
}

\author{
FLORA DI DONATO ET PASCAL MAHON
}

\begin{abstract}
e chapitre a pour objet de reconstruire la genèse de la notion d'intégration dans l'ordre juridique suisse, dans une perspective sociohistorique ${ }^{1}$. En lien avec notre première hypothèse exploratoire (1.4.1), nous analyserons ici quelles sont les différentes mesures législatives et administratives adoptées au fil du temps pour régler la présence des étrangères et des étrangers en Suisse. Nous verrons ainsi que les procédures de naturalisation se sont progressivement transformées de simples démarches de validation de la légitimité d'une demande (octroi automatique de la nationalité, ou «incorporation" par droit du sol) en procédures de transformation de la personne (par le biais de l'exigence d' "assimilation» et, plus tard, d' "intégration»).

Le chapitre est articulé comme suit. Dans un premier temps, il reviendra brièvement sur le débat politique et juridique actuel et sur l'émergence progressive de la notion d'intégration dans l'ordre juridique suisse, pour proposer quelques observations introductives relatives notamment à la méthodologie (3.1). Par la suite, il se propose de retracer les principales étapes politico-législatives qui ont conduit à la genèse de la notion d'intégration, en se fondant essentiellement sur la lecture de certains rapports et messages du Conseil fédéral (3.2). Une brève conclusion offrira une synthèse de cette évolution et permettra de faire le lien avec le chapitre suivant (3.3).
\end{abstract}

\subsection{OBSERVATIONS INTRODUCTIVES ET MÉTHODOLOGIQUES}

Le concept d'intégration occupe un rôle de premier plan dans le débat politique de nombreux pays, dont la Suisse. Devenu central

1. Ce chapitre, comme le suivant, propose une version synthétique et retravaillée de l'étude de Di Donato, 2016, qui constituait elle-même le premier résultat concret du projet de recherche dont le présent ouvrage est l'aboutissement. 
dans les politiques publiques à partir des années 1990, le thème de l'intégration s'est même imposé récemment comme une réalité juridique et normative incontournable, à l'occasion des révisions de deux actes législatifs fondamentaux en matière de politique migratoire: d'une part, la loi fédérale sur l'acquisition et la perte de la nationalité suisse, du 29 septembre 1952 (loi sur la nationalité, LN), révisée partiellement, pour ce qui nous intéresse ici, en 1990, ainsi qu'en 2003, 2004 et 2007, puis objet d'une révision totale en 2014; d'autre part, la loi fédérale sur les étrangers, du 16 décembre 2005 (loi sur les étrangers, LEtr), fruit d'une révision totale de l'ancienne loi sur le séjour et l'établissement des étrangers (LSEE), du 26 mars $1931^{2}$. Un des objets de ces diverses révisions était d'introduire, puis de renforcer et, en dernier lieu, d'harmoniser le concept d'intégration dans ces différents textes législatifs.

Si l'on prend la peine de parcourir le débat politico-juridique suisse depuis la fin du XIX ${ }^{\mathrm{e}}$ siècle, on peut constater que la thématique de l'intégration commence à prendre forme vers 1950, époque durant laquelle les flux migratoires deviennent importants ${ }^{3}$. Par la suite, dans les années 1960-1970, cette thématique sera abordée, de manière explicite, en lien avec la réglementation du statut des travailleurs étrangers sur le territoire suisse, et elle coïncidera avec diverses initiatives populaires dirigées contre les étrangères et les étrangers. À partir des années 1990, le terme même et le critère de l'intégration apparaissent officiellement, tout d'abord dans la loi sur la nationalité (art. 14), en tant que condition pour prétendre à la naturalisation, puis dans les premières lois cantonales spécifiques - comme la loi sur l'intégration et la cohésion multiculturelle du canton de Neuchâtel du 26 août 1996 ${ }^{4}$. Dans le droit fédéral des étrangers, la notion apparaît d'abord de manière timide dans la loi sur le séjour et l'établissement des étrangers ${ }^{5}$.

2. Tant la nouvelle LEtr que l'ancienne LSEE ont fait l'objet, chacune, de plusieurs révisions partielles, notamment, pour la première, en 2008, en 2009 et en 2012. Pour les révisions plus récentes de cette nouvelle loi sur les étrangers, voir plus bas dans le texte (notamment au chiffre 3.2.6 in fine).

3. Pour le débat historico-politique, voir Niederberger, 2005, pp. 257 ss. Selon cet auteur, on pourrait faire remonter la naissance d'une politique migratoire à l'entrée en vigueur de la loi fédérale du 26 mars 1931 concernant le séjour et l'établissement des étrangers (LSEE).

4. RSN 132.04 .

5. À travers l'art. 25 a de ladite loi, introduit par une révision du 26 juin 1998, dans le but de permettre à la Confédération de verser des subventions aux cantons en faveur de "l'intégration sociale des étrangers"; voir RO 1999 1111-1117. Cette disposition a été concrétisée par une première ordonnance du Conseil fédéral sur l'intégration des étrangers (OIE), du 13 septembre 2000, RO 2000 2281-2286. 
C'est avec l'approbation de la nouvelle loi fédérale sur les étrangers (LEtr), en 2005, qu'elle trouve une définition légale, à l'article $4^{6}$. Cette disposition, inspirée des principes de tolérance et de respect réciproque entre populations suisse et étrangère, propose une vision duale de l'intégration, mettant l'accent à la fois sur la disponibilité des étrangères et des étrangers à s'intégrer et sur une attitude d'ouverture de la population suisse.

Les pages qui suivent visent donc à proposer un travail de reconnaissance et de contextualisation ${ }^{7}$ des significations de l'intégration dans l'ordre juridique suisse ${ }^{8}$, en commençant par une perspective historique. Dans son effort de reconstituer la genèse $\mathrm{du}$ concept d'intégration, ainsi que ses significations du point de vue des autorités, ce chapitre se fonde principalement sur des documents officiels, en particulier sur les rapports et messages du Conseil fédéral à l'appui des différents projets normatifs qui se sont succédé, les uns avec succès, les autres sans, dans la mesure où ces projets ont été adoptés et transformés en lois entrées en vigueur ou, au contraire, ont été refusés, par le Parlement ou, parfois, par le peuple, en référendum. L'étude de ces sources officielles sera en outre confrontée à quelques avis de doctrine qui, dans le débat politique ou scientifique, ont accompagné les transformations politico-législatives au cours du XX $\mathrm{XX}^{\mathrm{e}}$ siècle.

Ainsi, pour comprendre l'évolution sociale, politique et juridique qui a conduit au modelage du concept d'intégration dans ses différentes significations, nous adopterons une double clé de lecture. D'une part, nous nous fonderons sur l'approche historique de Studer, Arlettaz et Argast (2013), selon laquelle les débats politiques reflètent l'imaginaire propre à un groupe social (représentations collectives, idéologies), le processus de législation étant conçu comme une pratique discursive qui repose sur des schémas de pensée et d'interprétation, associés à des schémas d'action, typiques d'un moment historique déterminé ${ }^{9}$. D'autre part, nous nous inspirons aussi de l'approche de Robert Cover, qui identifie dans les narrations législatives (dont fait partie la Constitution)

6. Pour le débat juridique sur le concept d'intégration, voir notamment Amarelle (éd.), 2012, en particulier les contributions de Facchinetti, pp. 61-80, Fibbi, pp. 81-94, et Gutzwiller, pp. 131-147. Voir aussi le récent ouvrage de Campisi, 2014.

7. En ce qui concerne le sens de l'expression «contextualisation du droit » à laquelle nous nous référons pour le présent chapitre, voir Gutwirth, 2013, p. 116.

8. Pour un examen de la signification du terme «intégration » en matière de politique des étrangers, voir par exemple Boccagni et Pollini, 2012, pp. 64 ss.

9. Studer, Arlettaz et Argast, 2013, pp. 7-44, en particulier les pages 35 ss. 
un facteur de cohésion sociale. Le processus que Cover qualifie de iurisgenesis a une matrice culturelle: les significations juridiques ne sont pas produites automatiquement d'en haut, mais sont le résultat d'un dialogue entre les institutions et les forces sociales. Elles trouvent ainsi dans la collectivité une forme de légitimation sociale ${ }^{10}$. À la lecture des rapports et messages du Conseil fédéral, on trouve les signes d'une telle dynamique de création de significations. On les trouve quelquefois dans le rôle d'équilibrage joué par le gouvernement fédéral vis-à-vis des poussées provenant du bas, comme on le voit avec les initiatives populaires des années 1960 et 1970 . On les trouve parfois aussi dans une certaine aptitude de la politique fédérale à faire levier, à certains moments - comme à l'époque de l'entre-deux-guerres -, sur la "capacité d'assimilation" de la population autochtone, ainsi que sur de «judicieuses mesures de police» pour faire face à ce qu'il était convenu d'appeler la "question des étrangers" ${ }^{11}$.

L'objectif du parcours de reconstruction analytique de ce chapitre est ainsi de retracer, à travers la lecture des documents officiels, la dynamique iurisgénérative qui s'est instaurée entre le haut (le législateur, le Conseil fédéral) et le bas et qui a modelé le $\mathrm{XX}^{\mathrm{e}}$ siècle, par la médiation entre les instances conservatrices ou "assimilationnistes» et les instances libérales ou progressistes. Nous pouvons en effet faire l'hypothèse que les significations courantes de l'intégration se sont configurées dans le temps comme le résultat de compromis d'une politique oscillant entre tendances assimilationnistes et libérales, pratiquée par le gouvernement suisse à l'égard des étrangers, en fonction aussi des flux migratoires.

\subsection{GENÈSE ET ÉVOLUTION DE LA NOTION D'INTÉGRATION DANS L'ORDRE JURIDIQUE SUISSE}

$\mathrm{La}$ reconstruction que nous proposons ci-après des principales étapes du débat politico-législatif suisse sur la "question des étrangers" se fonde sur différentes sources: d'un côté, comme nous l'avons dit, les documents officiels que constituent les messages et rapports du Conseil fédéral, ainsi que les textes constitutionnels et légaux; de l'autre, les contributions de la doctrine (histoire,

10. Cover, 1983-84, pp. 4-5 et 10.

11. Sauser-Hall, 1914, cité par Studer, Arlettaz et Argast, 2013, p. 38. 
géographie, science politique et droit public). Sur la base de cette reconstruction, nous avons identifié plusieurs étapes ou périodes de cette histoire, à savoir :

- 1848-1890, où la Suisse est pays d'émigration;

- 1890-1920, où il est surtout question d' "incorporation" des étrangers, par le biais de l'introduction du principe du droit du sol (ius soli), visant à faire de l'étranger un national;

- 1920-1950, période coïncidant avec l'introduction des permis de séjour et d'établissement;

- 1950-1975, où apparaît l'idée de l' "aptitude» de la personne étrangère à devenir citoyenne ;

- 1976-1990, où il est désormais question de l'«intégration» des travailleurs étrangers;

- 1990-2015, enfin, où l'intégration apparaît comme une tâche de l'État, une politique publique, caractérisée par une vision duale, consistant à encourager et, en même temps, à exiger l'intégration.

Nous reprendrons donc ici, de manière plus détaillée, ces diverses périodes de l'évolution.

\subsubsection{8-1890: LA SUISSE, PAYS D'ÉMIGRATION}

Il n'est pas inutile de rappeler que la Suisse a été, jusqu'en 1880 environ, un pays que l'on peut caractériser comme pays d'émigration, cela notamment sous l'effet de la crise économique provoquée par la révolution industrielle depuis le début du XIX ${ }^{\mathrm{e}}$ siècle. À cette époque, en effet, un des thèmes dominants des débats parlementaires internes était celui de la protection des Suissesses et des Suisses à l'étranger. Parallèlement, la législation d'autres pays, surtout celle des États-Unis ${ }^{12}$, est prise en exemple pour régler la présence des étrangères et des étrangers sur le territoire suisse ${ }^{13}$. C'est ainsi qu'en 1870, le Conseil fédéral a commencé à se doter de certaines compétences en matière de réglementation du statut des étrangères et des étrangers, par exemple, celle d'évaluer les liens de la personne avec son pays d'origine. À l'époque précédant la Constitution de 1848 , en effet, ce sont les cantons et les communes qui étaient compétents pour décider qui pouvait bénéficier de la citoyenneté,

12. Voir, par exemple, les recommandations et conseils pour les immigrants et immigrantes qui arrivaient à New York, émis par le Consulat suisse de cette ville et transmis au Conseil fédéral (extrait des délibérations du Conseil fédéral du 6 juillet 1853, FF 1853 II 876).

13. Voir aussi les extraits des délibérations du Conseil fédéral du 9 mars 1855, FF 1855 I $171,172-173$. 
conçue comme un «droit de bourgeoisie» ${ }^{14}$. Or, la Constitution fédérale de 1848 prévoit à son article 42 (première phrase) que " $[\mathrm{t}]$ out citoyen d'un canton est citoyen suisse» ${ }^{15}$. À partir de ce moment, quand bien même la compétence de décider en matière de naturalisation demeure cantonale, le Conseil fédéral s'accorde le droit de donner un préavis sur la personne de la candidate ou du candidat. À l'occasion des travaux de révision de la Constitution fédérale, en 1870, on en vient ainsi à imaginer deux actes distincts dans le cadre de la procédure de naturalisation: d'une part, la Confédération est compétente pour évaluer que «le candidat se libère des liens qui le rattachent à son pays d'origine» (compétence en matière de relations internationales), le canton étant d'autre part compétent pour évaluer qu' «il forme des nouveaux liens» (avec la communauté indigène). Il appartient en outre au canton d'apprécier la "valeur» morale de la candidate ou du candidat: "Les conditions dans lesquelles se trouve le candidat (valeur personnelle, position et état de fortune, conditions de famille) et ses rapports avec sa nouvelle patrie forment la partie essentielle de l'enquête à laquelle il doit être procédé», enquête menée par le canton ${ }^{16}$.

L'appréciation de ces exigences - de la valeur morale des candidates et candidats, y compris de leurs liens avec le pays d'origine - laisse déjà entrevoir les signes avant-coureurs d'une politique assimilationniste qui se développera une vingtaine d'années plus tard à travers un éventail de mesures visant à «transformer» la personne étrangère, comme on le verra.

La révision totale de la Constitution fédérale de 1874 donne à la Confédération un droit de regard sur les naturalisations, et lui permet d'en fixer les conditions minimales (art. 44) - y compris la réglementation de la renonciation à la nationalité suisse -, laissant pour le reste inchangées les compétences des cantons ${ }^{17}$. La Constitution de 1874 ne s'occupe en effet pas de réglementer l'entrée, le séjour et la résidence des étrangères et des étrangers

14. Sur le droit cantonal en matière de citoyenneté, voir Gutzwiller, 2008, pp. 107 ss.

15. Pour le texte de la Constitution fédérale du 12 septembre 1848, voir FF 1849 I 3-34. En matière de naturalisation, l'art. 43 précisait uniquement que les «étrangers ne peuvent être naturalisés dans un canton qu'autant qu'ils seront affranchis de tout lien envers l'État auquel ils appartenaient», laissant pour le reste entièrement aux cantons la compétence de décider.

16. Citations extraites du Message du Conseil fédéral à l'Assemblée fédérale touchant la révision de la Constitution fédérale, du 17 juin 1870 (FF 1870 II 777, 793-797).

17. Voir Mahon, 2003, ad art. 38, no 1, p. 345. Voir également Grisel, 1989, ad art. 44, no 3 , p. 4. Selon cet auteur, avec la réforme de l'art. 44, la Confédération entend mettre un frein à certains abus de la part des cantons. 
(ce qui sera prévu ultérieurement par l'art. 69ter) : elle accorde simplement à la Confédération «le droit de renvoyer de son territoire les étrangers qui compromettent la sûreté intérieure ou extérieure de la Suisse» (art. 70 Cst. 1874) ${ }^{18}$. En vertu des pouvoirs qui lui sont conférés, la Confédération peut en outre intervenir pour conclure avec les États étrangers des traités d'établissement qui concèdent aux citoyennes et citoyens des États cocontractants un droit de s'établir librement dans le pays et d'y exercer l'activité lucrative de leur choix. Cette phase de la politique fédérale est définie comme libérale ${ }^{19}$. D'autre part, comme cela est documenté par plusieurs sources, durant cette période, les cantons eux-mêmes pratiquent une politique relativement libérale à l'égard des étrangères et des étrangers, au point que le rapport entre la proportion de ceux-ci et celle des personnes de nationalité suisse sur le territoire est, jusqu’à la veille de la Première Guerre mondiale, d'un à six ${ }^{20}$. En 1876, la proposition du Conseil fédéral de répartir les compétences en matière de naturalisation entre la Confédération et les cantons se transforme en projet de loi, puis en loi. Le projet prévoit que les étrangers et les étrangères qui souhaitent acquérir la nationalité suisse s'adressent d'abord au Conseil fédéral pour obtenir une autorisation leur permettant «de se faire recevoir bourgeois d'un canton et d'une commune» (droit de bourgeoisie); il appartient aux cantons et communes qui veulent concéder ("faire don» de) ce droit de bourgeoisie de demander l'autorisation au Conseil fédéral, par le biais du Gouvernement cantonal. Le Conseil fédéral accorde l'autorisation aux étrangères et étrangers qui remplissent les conditions suivantes: avoir établi leur domicile ordinaire en Suisse depuis une année au moins et s'être libérés de tous liens et obligations à l'égard du pays d'origine, de manière à éviter tout conflit en cas d'obtention de la nationalité suisse ${ }^{21}$. C'est sur la base de ce projet, et avec quelques modifications terminologiques, que voit le jour, le

18. Sur ce point, voir Malinverni, 1995, ad art. 69ter, no 1, p. 4, ainsi que 1987, ad art. 70, nos 9 à 11, p. 3. Cf aussi Mahon, 2003, ad art. 121, no 2, p. 960.

19. Voir Argast, 2013, pp. 45-76. Selon Niederberger (2005, p. 256), une telle politique libérale est déterminée par une série de traités bilatéraux conclus entre la Confédération et d'autres États européens, dans un contexte plus général de libéralisme économique émergent. L'attitude libérale de la Confédération est confirmée aussi par Piguet, 2009, p. 12. Gadient (2015, pp. 15-32) démontre cependant comment, dans cette phase, les expulsions ont pu fonctionner comme instrument de contrôle de l'immigration.

20. Cf. Malinverni, 1995, ad art. 69ter, n 2, p. 4, et Aubert, 1993, pp. 361 ss.

21. Voir le Message du Conseil fédéral à la haute Assemblée fédérale concernant le projet de loi sur la naturalisation des étrangers en Suisse et la renonciation à la nationalité suisse, du 2 juin 1876, ainsi que le projet de loi qui s’y réfere, FF 1876 II 940, 948-950. 
3 juillet 1876, la première loi fédérale sur la naturalisation suisse et la renonciation à la nationalité suisse ${ }^{22}$.

\subsubsection{0-1920: L'INCORPORATION PAR LE BIAIS DU «IUS SOLI»}

Dès 1900 environ, la présence d'immigrées et immigrés de nationalité étrangère sur le territoire suisse commence à augmenter de manière significative et, en 1915 , la population étrangère dépasse les $15 \%$ de la population résidente ${ }^{23}$. Durant cette période, le débat porte sur la nécessité de limiter le nombre d'étrangers et d'étrangères, contexte dans lequel est aussi rediscutée la répartition des compétences entre la Confédération et les cantons et la question de la façon dont il convient d'assimiler la population étrangère. C'est à cette époque que l'on fait généralement remonter l'apparition du terme d'Überfremdung ${ }^{24}$.

Dans son message à l'Assemblée fédérale concernant la révision de la loi fédérale sur la naturalisation suisse et la renonciation à la nationalité suisse du 20 mars $1901^{25}$, le Conseil fédéral présente un nouveau projet de loi qui prévoit que la candidate ou le candidat doit adresser au Conseil fédéral la demande «de se faire recevoir citoyen d'un canton ou d'une commune»; le gouvernement cantonal qui entend accorder la naturalisation doit en requérir l'autorisation auprès du Conseil fédéral. Celle-ci suppose que l'étrangère ou l'étranger ait "eu son domicile ordinaire en Suisse pendant les deux ans qui précèdent immédiatement sa demande»; de plus, il appartient au Conseil fédéral d'évaluer les liens entretenus avec son pays d'origine, ainsi que toutes les autres circonstances relatives à sa personne et à sa famille; l'autorisation peut être refusée lorsque la naturalisation risquerait de porter préjudice à la Confédération. Sont nulles toutes les décisions cantonales et communales qui ne seraient pas assorties de l'autorisation. De la même manière, la nationalité suisse n'est pas acquise

22. FF 1876 III 465-468. En ce qui concerne la terminologie, les termes «bourgeoisie» et «bourgeois» deviennent "citoyenneté" et "citoyen».

23. Sur ce point, voir Piguet, 2009, p. 12.

24. Cf. Arlettaz, 2013, p. 77. Voir aussi Studer, 2013, pp. 117-177. Pour approfondir, voir en particulier Arlettaz, 2013, pp. 77 ss. Niedeberger (2005, p. 258) précise que le terme Überfremdung est traduit comme «invasion de la Suisse de la part des étrangers», ou parfois comme "emprise étrangère" ou "surpopulation étrangère». Il faut noter que le concept de Überfremdung ne se limite pas seulement à une «surpopulation " comme fait démographique, mais comprend aussi tout ce qui donne forme à la nationalité et à la spécificité suisse.

25. FF 1901 II 769-800. 
si l'autorisation fédérale n'est pas suivie par une naturalisation cantonale et communale ${ }^{26}$.

L'approbation, le 25 juin 1903, d'une nouvelle loi fédérale sur la naturalisation des étrangers et la renonciation à la nationalité suisse marque ainsi un renforcement des pouvoirs de la Confédération en matière de naturalisations ${ }^{27}$. Le Conseil fédéral poursuit par ailleurs une politique de réciprocité et d'égalité de traitement entre personnes étrangères et suisses, reconnaissant d'un côté que les premières contribuent au bien-être général du pays et, de l'autre, que la Suisse compte déjà un grand nombre de personnes expatriées à l'étranger. En 1916, la Division des affaires intérieures constate que l'éclatement de la guerre a entraîné une forte augmentation des demandes de naturalisation suisse. En conséquence, il est question d'augmenter d'urgence de deux à cinq ans la durée exigée du domicile effectif en Suisse et la demande de révision de la loi sur les naturalisations est pressante: il est requis du Conseil fédéral qu'il en accélère les travaux préparatoires ${ }^{28}$. Sur un plan plus général, la fermeture des frontières consécutive à l'éclatement du conflit induit le Conseil fédéral à adopter, le 21 novembre 1917, une ordonnance sur le contrôle des étrangers, qui subordonne leur entrée en Suisse à l'obtention d'un visa fédéral. La même année est créé un office central de police des étrangers, qui trouvera sa base constitutionnelle en 1925, dans le nouvel article 69ter de la Constitution. Dorénavant, l'opinion publique tendra à considérer que la candidate ou le candidat doit déjà être assimilé avant de - et pour - pouvoir être naturalisé. Se développera ainsi, à l'échelle nationale, une conception selon laquelle, dans une certaine mesure, il y a lieu de protéger le fonctionnement économique et l'équilibre social et culturel du pays au regard d'influences qui pourraient altérer «le sentiment national identitaire et les vertus propres aux Confédérés» ${ }^{29}$. La naturalisation finit par devenir un élément de "gestion du social» et un instrument d'une forme de défense identitaire ou culturelle qui se traduit par une intense activité intellectuelle,

26. Voir le projet de loi fédérale sur la naturalisation des étrangers et la renonciation à la nationalité suisse, annexé au message précité, FF 1901 II 769, 801 ss.

27. Le texte de la loi adoptée est publié à la FF 1903 III 939-945.

28. Voir le Rapport de la commission du Conseil national sur la gestion du Conseil fédéral et du Tribunal fédéral en 1915, du 19 mai 1916, FF 1916 III 107-154, 109.

29. Cf. Arlettaz, 2013, p. 81. 
juridique, politique et médiatique ${ }^{30}$. Entre 1910 et 1920, la question de la naturalisation est considérée comme vitale pour la Suisse, selon les termes mêmes d'un expert de droit international comme Georges Sauser Hall, qui propose divers remèdes en termes d'assimilation des étrangers ${ }^{31}$ :

[...] pour résoudre la question des étrangers, l'État ne doit pas se contenter de naturaliser les immigrés par le jeu automatique de ses lois; il doit employer tous les moyens - petits et grands, aucun n'est à mépriser - pour rétablir une proportion plus normale entre la population nationale et allogène. Ces moyens sont de deux sortes: les premiers, de nature préventive, doivent entraîner une diminution de l'immigration; les seconds doivent faire un national de l'immigré; ceux-ci seront à leur tour, ou bien moraux afin de faciliter l'assimilation des étrangers, ou bien législatifs et destinés à rendre notre droit de cité plus facilement accessible à nos hôtes.

La population autochtone est appelée en cause comme partie active du processus d'assimilation ${ }^{32}$ :

Ces étrangers, nous devons les métamorphoser en nationaux; et c'est affaire de la loi de déclarer lesquels d'entre eux devront faire partie de notre corps public; mais c'est affaire de l'âme d'insuffler à ces nouveaux citoyens un peu de l'amour civique qui nous anime, de les gagner à nos idées de tolérance et de solidarité, à nos traditions démocratiques.

Dans l'intervalle, le 9 novembre 1920, le Conseil fédéral présente à l'Assemblée la nécessité de revoir l'article 44 de la Constitution, suite aux manifestations, dans le pays, sur l'opportunité de réformer la loi sur la naturalisation et l'introduction de mesures destinées à faciliter cette naturalisation, en transformant les étrangères et les

30. Ce qui sera confirmé dans les messages plus récents du Conseil fédéral; voir par exemple le Rapport du Conseil fédéral sur la politique à l'égard des étrangers et des réfugiés, du 15 mai 1991, FF 1991 III 316-348, où sont reconstruites les étapes de la politique fédérale concernant les étrangers.

31. Le passage est tiré de Sauser-Hall, 1914, p. 180.

32. Ibid., p. 3. Voir aussi Sauser-Hall, 1921. 
étrangers en "nationaux» ${ }^{33}$. Une révision partielle de la législation est particulièrement attendue pour éviter les naturalisations "peu sincères" et modifier la législation sur l'indigénat. La tonalité du message est toutefois partagée entre les arguments en faveur des étrangères et des étrangers et les craintes ou les risques inhérents à leur présence trop importante. Parmi les arguments en faveur est évoqué le rôle toujours plus important qu'ils jouent dans le développement de l'économie du pays ${ }^{34}$. Mais certains passages du message mettent aussi l'accent sur le risque d'une "dénationalisation", sur la transformation des valeurs et des coutumes, etc. ${ }^{35}$ :

Notre peuple doit résister à des influences qui lentement risquent de le dénationaliser. Tout peuple fort et sain peut et doit vivre d'échanges actifs avec l'étranger tant dans le domaine moral et politique que dans le domaine matériel; mais un certain équilibre ne doit pas être rompu; lorsque le fléau de la balance incline trop en faveur de l'étranger, il y a péril pour un peuple d'aliéner son individualité et de devenir non seulement matériellement, mais intellectuellement tributaire d'autres États. Les mœurs, les usages, les idées politiques, les valeurs morales elles-mêmes et surtout les sentiments de dévouement à la patrie risquent alors de se modifier, de s'estomper.

Dans ce même message, au titre IV intitulé «Insuffisance de la politique de naturalisation", le Conseil fédéral fait appel à l'influence assimilatoire de la population suisse ${ }^{36}$ :

Les mesures à prendre par le législateur doivent viser à faciliter l'assimilation des étrangers, à désigner les éléments assimilables ensuite de l'influence que l'ambiance suisse a exercée sur eux, à permettre au peuple suisse, par une action continue, d'exercer une action décisive sur l'esprit et les mœurs de nos hôtes.

33. Du reste, comme le précisent Silvia et Gérald Arlettaz, l'objectif n'est plus, après la Première Guerre mondiale, d'assimiler les étrangers, mais de les contrôler. Voir Arlettaz et Arlettaz, 2004, p. 12.

34. Voir le Message du Conseil fédéral à l'Assemblée fédérale concernant la révision de l'article 44 de la Constitution fédérale (mesures à prendre pour assurer l'assimilation des étrangers en Suisse), du 24 novembre 1920, FF 1920 V 1-74, qui vise à renforcer le pouvoir de la Confédération en matière de naturalisation.

35. Ibid., p. 17.

36. Ibid., p. 18. 
Il en résulte que, pour éviter cette perte d'identité nationale, il faut non seulement diminuer l'immigration, mais aussi incorporer les personnes étrangères, au moins celles qui sont "assimilées ou assimilables ", c'est-à-dire nées en Suisse ou qui y résident depuis longtemps. Ce groupe forme, ensemble, plus de $50 \%$ de la population de nationalité étrangère dans le pays, d'où la proposition d'introduire dans l'article 44 de la Constitution des éléments de ius soli et de faciliter la naturalisation ${ }^{37}$ :

«Il faut donc songer à incorporer à l'État les personnes qui, chaque année, viennent augmenter les contingents étrangers ", de manière à «faire un national de l'immigré et agir sur la population étrangère déjà établie en Suisse", notamment par l'«introduction de l'incorporation iure soli dans notre économie législative», qui «doit être la mesure capitale de notre politique d'assimilation des étrangers».

Dans cette phase, donc, malgré la crainte d'une menace identitaire due à l'augmentation de la présence d'une population étrangère, l' «incorporation" des étrangères et des étrangers est perçue comme un remède propre à rééquilibrer la relation entre populations suisse et étrangère. La naturalisation devient ainsi un instrument de gestion du social. Ce que nous avons défini comme une institution de réinvention ou de transformation de la personne (1.4.1) commencera à prendre forme non seulement à travers l'adoption de mesures de nature politico-législative, mais aussi de mesures qui ont un impact social. L'assimilation des étrangères et des étrangers ne peut s'effectuer sans l'œuvre active de la population locale, qui devra exercer une action décisive sur l'esprit et sur les coutumes des personnes étrangères. Si les besoins de l'économie ne permettent pas de se passer des étrangères et des étrangers, il faut au moins trouver le moyen de sauvegarder les us et coutumes du pays.

Toutefois, l'intégration comme conséquence de la naturalisation automatique subira un brusque coup d'arrêt et sera rapidement abandonnée au moment où, en juin 1922, est rejetée l'initiative populaire qui visait à conférer de plein droit la nationalité aux étrangères et aux étrangers qui sont nés et ont grandi en Suisse ${ }^{38}$.

37. Ibid., pp. 19, 29 et 37.

38. L'initiative visait à faciliter la naturalisation des étrangers nés et ayant grandi en Suisse à travers une législation fédérale qui leur aurait reconnu la nationalité ipso iure. Elle a été rejetée par le peuple et les cantons le 11 juin 1922. Voir à ce propos Gutzwiller, 2008, pp. 127-128. 
Ce rejet marque un pas en arrière en relation avec la volonté d'introduire le principe du ius soli et la naturalisation automatique pour les étrangers de deuxième et troisième générations.

Dans son message complémentaire du 14 novembre 1922, établi à la demande de la commission du Conseil des États à la suite du recensement de $1920^{39}$, mais postérieur à la votation sur l'initiative populaire en question, le Conseil fédéral répète certes que, pour résoudre le problème devenu urgent de «l'envahissement de la population allogène [...] il est indispensable d'introduire la naturalisation iure soli (par incorporation) » pour les enfants nés en Suisse ${ }^{40}$. Il propose ainsi une révision en ce sens de l'article 44 de la Constitution, fondée sur trois points essentiels. Premièrement, la législation fédérale réglera les conditions d'acquisition et de perte de la nationalité suisse. Deuxièmement, durant les cinq ans qui suivent leur naturalisation, les personnes naturalisées ne seront pas éligibles au sein des autorités législatives et exécutives fédérales et cantonales. Et enfin, troisièmement, la loi pourra prévoir l'incorporation par ius soli, en particulier pour les enfants étrangers nés en Suisse de parents étrangers dont la mère était suisse par naissance ${ }^{41}$. L'accent est donc de nouveau mis sur la présence invasive des étrangères et des étrangers, et sur la lutte contre une telle invasion, en tant que tâche de l'État, dont la naturalisation iure soli est présentée comme le remède. Mais, par rapport au projet précédent, le pas en arrière est clair et réside dans le fait que seule est désormais proposée la naturalisation iure soli des enfants eux-mêmes nés en Suisse - et non plus dont les parents sont domiciliés en Suisse ou sont nés en Suisse -, de mère étrangère d'origine suisse de par sa naissance ${ }^{42}$.

Le nouvel article 44 de la Constitution, adopté par les Chambres fédérales le 30 septembre $1927^{43}$, sera finalement approuvé par le peuple et les cantons le 20 mai 1928, dans une formulation quelque peu plus large: il permet à la loi de «statuer que l'enfant né de parents étrangers est ressortissant suisse, dès sa naissance, lorsque la mère était d'origine suisse par filiation et que les parents

39. FF 1922 III 683-698.

40. Ibid., p. 694.

41. Ibid., pp. 697-698.

42. Le projet de 1920 prévoyait en effet l'incorporation iure soli aussi des enfants nés à l'étranger de parents légalement domiciliés en Suisse et des enfants dont le père ou la mère étaient nés en Suisse; voir FF 1922 III 694-695.

43. Sur la base des deux messages évoqués, de 1920 et de 1922; voir, pour le texte de l'arrêté fédéral, FF 1927 II 261-262. 
sont domiciliés en Suisse au moment de la naissance de l'enfant » ${ }^{44}$. Cette norme ne sera toutefois concrétisée que beaucoup plus tard, "d'abord timidement par la nouvelle loi fédérale sur l'acquisition et la perte de la nationalité suisse, du 29 septembre 1952, puis surtout par une révision de cette dernière, dans le cadre de l'adoption du nouveau droit de la filiation, le 25 juin $1976 »{ }^{45}$.

\subsubsection{0-1950: L'INTRODUCTION DES PERMIS DE SÉJOUR}

\section{ET D'ÉTABLISSEMENT}

Dans l'intervalle, en 1925, l'adoption de l'article 69ter de la Constitution attribue à la Confédération, de manière définitive, la compétence de réglementer l'entrée, la sortie, le séjour et l'établissement des étrangères et étrangers en général, laissant aux cantons le pouvoir de décision, sans toucher le système de la nationalité à trois niveaux (communal, cantonal et fédéral) ${ }^{46}$. La Confédération a en outre le droit de décider en dernière instance dans certains cas, à savoir dans l'hypothèse de la potentielle violation des traités d'établissement et celle du refus d'accorder l'asile. Sur ces bases, la Confédération adoptera, peu après, la loi fédérale du 26 mars 1931 concernant le séjour et l'établissement des étrangers (LSEE), qui est restée en vigueur jusqu'à la fin de $2007^{47}$. Cette loi, qui marque la création d'un dispositif central de contrôle et de réglementation de la politique à l'égard des étrangères et étrangers, définira en outre ultérieurement les conditions de leur présence sur le territoire.

En effet, à partir de ce moment, cette présence ne sera plus seulement réglementée à travers le mécanisme de la naturalisation, mais aussi par le biais d'une politique de permis. De cette manière, il est possible de mettre un frein à l'affluence des étrangères et étrangers durant les années 1930 et pendant l'entre-deux-guerres, et d'introduire une législation-cadre qui laisse au Conseil fédéral

44. Voir le Message du Conseil fédéral à l'Assemblée fédérale relatif à la votation populaire du 20 mai 1928 sur l'arrêté fédéral du 30 septembre 1927 concernant la révision de l'article 44 de la Constitution (naturalisations), du 15 juin 1928, FF 1928 II 165-168.

45. Voir Mahon, 2003, ad art. 38, no 3d, p. 346. Voir aussi, sur la portée de la modification de l'art. 44, qui n'élargit que peu les compétences de la Confédération, Grisel, 1989, ad art. 44, nos 3 et 4, p. 4, et Gutzwiller, 2008, p. 133.

46. Gutzwiller, 2008, pp. 133-134.

47. Voir aussi le Message du Conseil fédéral à l'Assemblée fédérale concernant un projet de loi sur le séjour et l'établissement des étrangers, du 17 juin 1929, FF 1929 I 929-949. Le projet prévoit trois types de statuts: les autorisations de séjour et d'établissement ainsi que la «tolérance». Il décrit en outre la procédure que l'étranger doit suivre dès son arrivée, notamment se présenter à la police des habitants du canton dans les trois mois dès son arrivée, etc. 
une large marge de manœuvre dans le contrôle de la politique démographique $^{48}$. Le critère autour duquel s'articule la loi sur le séjour et l'établissement des étrangers est essentiellement celui d'un rapport équilibré entre la population suisse et la population étrangère. Comme on peut le lire dans le préambule du projet de modification en 1948, la loi de 1931 a pour but de protéger le pays de la surpopulation étrangère et la main-d'œuvre suisse d'une concurrence excessive du personnel de nationalité étrangère. Un rôle clé dans le contrôle et la limitation du nombre de personnes étrangères est attribué à ce qu'il est désormais convenu d'appeler la police des étrangers ${ }^{49}$. Le projet de révision de la loi prévoit ainsi que l'autorité décide non seulement en fonction des documents de légitimation, mais aussi en fonction de la personnalité de l'étrangère ou de l'étranger, et il prévoit la possibilité de l'expulsion pour des motifs liés à sa conduite, à savoir dans le cas d'une non-adaptation à l'ordre établi dans le pays d'accueil. De cette façon, les conditions relatives à l'octroi des permis qui sont soumis à une approbation fédérale sont rendues plus strictes. Il s'agit d'une conception "plus individualisée du droit des étrangers ", qui finit par diviser ceux-ci en plusieurs catégories, dont certaines sont considérées comme peu assimilables et sont donc plus ou moins écartées ${ }^{50}$.

Ainsi, l'institution de réinvention se dote ici d'instruments complémentaires agissant non seulement à travers le contrôle de la naturalisation, mais aussi sur la base du système des permis de séjour et de la possibilité d'expulser les étrangères et les étrangers indésirables.

\subsubsection{0-1975: L'APTITUDE DE LA PERSONNE ÉTRANGÈRE}

\section{À DEVENIR CITOYENNE}

À la suite de la Seconde Guerre mondiale, en raison aussi de la diminution du nombre de personnes étrangères, le ton du débat en matière de politique migratoire semble changer : on passe d'une politique de défense des frontières territoriales, politiques et culturelles

48. Voir pour cette reconstruction Mahon, 2003, ad art. 121, nos 2 à 6, pp. 960-964, et Malinverni, 1995, ad art. 69ter, nos 3 à 6, p. 5. Sur la question, cf. aussi Studer, 2013, pp. 117-119. L'introduction de la LSEE, comme l'explique bien Garufo (2015, p. 110), est essentiellement liée à l'exigence de réglementer, au niveau fédéral, la présence des travailleurs frontaliers, question gérée jusqu'à ce moment-là par les cantons.

49. Voir le Message du Conseil fédéral à l'Assemblée fédérale concernant une loi modifiant et complétant la loi sur le séjour et l'établissement des étrangers, du 8 mars 1948, FF 1948 I 1277-1293.

50. Voir Mahon et Collette, 2011, p. 230. 
à la considération des rapports entre la Suisse et les autres États. Les immigrées et immigrés, vus jusque-là comme objets à assimiler, incorporer, accoutumer, ou à qui opposer résistance, commencent à être pris en compte en tant que personnes ${ }^{51}$. Ce changement au moins partiel de perspective coïncide avec une évolution plus vaste de la conception des rapports entre État et individus - à la suite des deux guerres - ainsi qu'avec le développement de la science juridique elle-même, laquelle avait jusqu'alors considéré l'individu comme privé de toute subjectivité. Le thème de la naturalisation s'inscrit dès lors dans le concept plus général des rapports entre État et individus. Ainsi qu'on peut le lire dans le message du Conseil fédéral à l'appui d'un nouveau projet de loi sur l'acquisition et la perte de la nationalité suisse, du 9 août 1951, la naturalisation répond non seulement aux besoins des individus, mais aussi aux exigences de l'État et, plus généralement, à la réglementation des relations entre États ${ }^{52}$ :

La nationalité prit beaucoup plus d'importance, pour l'État comme pour l'individu, à la suite de l'évolution politique et économique et particulièrement des deux guerres mondiales, avec leurs incommensurables bouleversements et leurs répercussions sur la vie internationale et nationale, publique et privée. Les frontières entre les pays prirent une signification plus manifeste que par le passé. Pour sauvegarder leur indépendance et leur patrimoine national, pour protéger leur population et ses conditions de vie, les États furent contraints de plus en plus de s'isoler les uns des autres. De nos jours, il y a bien peu de choses que l'individu puisse faire ou omettre - en dehors de sa vie privée - sans que, volontairement ou non, il entre en contact avec l'État et ses autorités, la nationalité jouant dans ces rapports un rôle important. Ce phénomène a pour signe extérieur la valeur que l'on attribue aujourd'hui - alors que ce n'était guère le cas autrefois - au passeport ou à l'acte d'origine.

51. Selon Studer, toutefois, même dans la phase qualifiée de «libérale», les forces conservatrices et nationalistes s'opposent à toute tentative de créer une "nation de volonté» et une "nation de souche». Reste en effet ouverte la question de savoir si la Suisse est un État créé par l'effet de son système démocratique ou par l'effet d'une culture commune suisse, et si la nationalité est créée généalogiquement ou territorialement (Studer, 2013, p. 118). Sur le thème de l'identité suisse et du fédéralisme de type "culturel», voir aussi Di Donato et Mahon, 2009, pp. 281-294.

52. Message du Conseil fédéral à l'Assemblée fédérale à l'appui d'un projet de loi sur l'acquisition et la perte de la nationalité suisse, du 9 août 1951, FF 1951 II 665-718, 672. 
Dans cette phase, comme cela avait déjà été le cas en 1920, le droit du sol (ius soli) est mis en relation avec la force assimilatrice de la population autochtone et avec de «judicieuses mesures de la police des étrangers ${ }^{53}$.

En 1952, le projet de loi sur la naturalisation est approuvé et devient la loi fédérale sur l'acquisition et la perte de la nationalité suisse (loi sur la nationalité, LN), du 29 septembre 1952, qui entre en vigueur le $1^{\text {er }}$ janvier $1953^{54}$. L'idée de la naturalisation comme instrument de lutte contre l'emprise étrangère perdurera jusqu'au début des années 1970. Entre 1960 et 1970, le thème de la surpopulation étrangère ou de l'emprise étrangère fera l'objet de nombreuses initiatives populaires. Si, jusque-là, le thème principal avait été l'assimilation des étrangères et des étrangers à travers le parcours obligatoire de la naturalisation, le problème devient celui de la réglementation de l'entrée et du séjour des travailleuses et des travailleurs étrangers, qui sont accueillis en nombre pour les besoins de l'économie, mais dont on n'attend pas de véritable intégration. Dans les années 1960, en effet, l'ouverture opérée par la Suisse à l'égard de la main-d'œuvre étrangère induit une forte augmentation démographique, qui provoque l'introduction d'un nouveau contrôle des entrées et une série de mesures restrictives de la part du Conseil fédéral. À partir de ce moment est introduit un système de contingentement du nombre des travailleuses et des travailleurs étrangers de la part de la Confédération et des cantons ${ }^{55}$.

Entre 1965 et 1974, la question de la présence étrangère est de nouveau perçue comme pressante. Le mouvement contre l'«emprise étrangère» lance pas moins de cinq initiatives populaires $^{56}$. C'est à l'occasion de la troisième initiative de l'Action nationale que le Conseil fédéral soulève la question de l'intégration. Dans son rapport à l'Assemblée fédérale du 21 décembre 1973, il recommande au Parlement de rejeter l'initiative, avec l'argument que la réduction de la population étrangère en dessous

53. Ibid., p. 677.

54. RO 19521115 (RS. 141.0).

55. Pour de plus amples approfondissements, voir Malinverni, 1995, ad art. 69ter, nos 3 à 6, p. 5, et pour les aspects concernant principalement le droit du travail et le système de contingentement des années 1970, cf. Mahon, 1984, pp. 122-123.

56. Sur le contenu de ces initiatives, dont la plus connue est l'initiative dite "Schwarzenbach", du nom d'un de ces auteurs, initiative "contre l'emprise étrangère", de 1970, cf. Piguet, 2009, pp. 23-26. Sur les tons plutôt violents des initiatives, qui semblent ramener au débat nationaliste et xénophobe de la Première Guerre mondiale, cf. Studer, 2013, p. 135. 
d'un certain taux serait désastreuse pour l'économie du pays et risquerait de compromettre les relations de la Suisse avec les autres États ${ }^{57}$. Tout en recommandant le rejet de l'initiative, le Conseil fédéral cherche à concilier les préoccupations des électeurs et, depuis 1971, des électrices face à l'augmentation croissante de la population étrangère en rappelant les exigences de l'économie et les exigences humanitaires, expliquant que sa limitation drastique, telle que requise par la précédente initiative populaire, ne saurait être admise ${ }^{58}$ :

Une diminution aussi radicale n'aurait pas été supportable, avant tout pour des motifs économiques; elle aurait en effet perturbé gravement l'économie, entraînant en particulier la fermeture d'un nombre important d'entreprises et affectant par là également le sort de travailleurs suisses. Menée à chef, l'initiative aurait en outre rendu impossible, dans de nombreux cas, la prise en considération des exigences de l'humanité.

C'est dans ce rapport qu'apparaît pour la première fois le terme d'intégration. Le Conseil fédéral rappelle les raisons pour lesquelles il fallait rejeter l'initiative populaire contre l'emprise étrangère, raisons qu'il attribue à la volonté du peuple suisse de résoudre de manière équilibrée les problèmes d'ordre culturel, humain et économique liés à un nombre important de personnes de nationalité étrangère. Le Gouvernement souligne, en même temps, les deux aspects proéminents de la politique à mener dans le futur en la matière: d'un côté, la limitation du nombre des travailleuses et travailleurs étrangers, de l'autre, leur «intégration sociale» et leur "assimilation ${ }^{59}$ :

Par cette déclaration, nous soulignions les deux aspects fondamentaux de notre politique future à l'égard des étrangers, à savoir, d'une part, la nécessité de limiter le nombre des travailleurs étrangers et, d'autre part, celle de résoudre les problèmes liés à l'intégration sociale et à l'assimilation des immigrés.

57. Rapport du Conseil fédéral à l'Assemblée fédérale sur la troisième initiative populaire contre l'emprise étrangère (initiative populaire contre l'emprise étrangère et le surpeuplement de la Suisse), du 21 décembre 1973, FF 1974 I 183-215.

58. Ibid., p. 186

59. Ibid., p. 188. 
Dans cette phase, l'intégration finira par devenir un but en soi, avec l'abandon d'une philosophie connotée par l'assimilation et les réflexes de peur et de défense ${ }^{60}$.

Dans les années 1950 à 1970, les transformations politiques et les conceptions juridiques émergentes au sujet des rapports entre État et individus font que les tendances à la transformation ou à la réinvention de la personne étrangère se réduisent. Celle-ci n'est plus considérée (seulement) comme objet des mesures étatiques, mais comme personne humaine, titulaire de droits. À la conception assimilationniste tend à se substituer une conception inclusive, qui vise à intégrer les travailleuses et les travailleurs étrangers et leur famille, en leur offrant aussi des garanties sur le plan social et en matière d'assistance.

\subsubsection{6-1990: L'INTÉGRATION DES TRAVAILLEUSES}

\section{ET DES TRAVAILLEURS ÉTRANGERS}

En 1976, à la suite de la récession économique due à la crise pétrolière, se vérifie une nouvelle vague de préoccupations face au phénomène migratoire, marquée par deux autres initiatives populaires, en 1976 et $1977^{61}$. En particulier, la quatrième initiative demande que «l'effectif des étrangers au bénéfice d'une autorisation d'établissement ou de séjour soit, pour l'ensemble de la Suisse, ramené en l'espace de dix ans à 12,50\% du nombre des ressortissants suisses dénombrés lors du dernier recensement de la population " ${ }^{62}$.

Dans son message sur cette initiative, le Conseil fédéral évalue à nouveau les conséquences d'une acceptation de celle-ci en utilisant une argumentation d'ordre économique (liée au besoin en termes de main-d'œuvre étrangère), d'ordre humanitaire, mais aussi en se référant aux relations avec les autres États. On peut ainsi lire dans ce message ${ }^{63}$ :

L'acceptation de l'initiative aboutirait par ailleurs à un régime incompatible avec la Convention européenne des droits de l'homme. Le renvoi de dizaines de milliers d'étrangers soumettrait en effet ceux qui seraient l'objet de cette mesure à un traitement

60. Voir Niedeberger, 2005, p. 278.

61. Voir à ce propos Piguet, 2009, pp. 33 ss.

62. Voir le Message du Conseil fédéral à l'Assemblée fédérale concernant l'initiative populaire du Parti républicain "pour la protection de la Suisse» (4 initiative contre l'emprise étrangère), du 8 mars 1976, FF 1976 I 1343-1380, en particulier 1857.

63. Ibid., p. 1366. 
inhumain et dégradant. Cela constituerait donc une atteinte aux dispositions de l'article 3 de cette convention.

Le thème de l'intégration est repris dans le même message en lien avec les exigences du marché du travail ${ }^{64}$ :

Comme nous l'avons déjà relevé dans notre rapport sur la première initiative contre la pénétration étrangère, le problème des étrangers ne peut pas être résolu uniquement par une pratique restrictive en matière d'admission des étrangers (FF 1967 II 103). En dépit de la situation actuelle défavorable de l'emploi, il faut partir de l'idée que notre économie continuera longtemps encore à avoir besoin de main-d'œuvre étrangère. Cela implique l'obligation de prévoir des dispositions qui favorisent l'intégration des étrangers dans notre communauté nationale, surtout de ceux qui séjournent depuis longtemps en Suisse avec leur famille ou se proposent de s'y établir à demeure. Encourager l'intégration est en effet l'un des éléments essentiels de la politique que nous suivons à l'égard des étrangers. L'État apporte sa propre contribution à cette tâche en améliorant le statut juridique de l'étranger.

Est ainsi proposée l'introduction de mesures qui permettront à la population étrangère de "mieux s'intégrer» et de «se sentir à l'aise en tant qu'étranger", sans pour autant renoncer à sa propre identité culturelle ${ }^{65}$ :

Outre l'amélioration progressive du statut juridique des étrangers, il faudra d'autres moyens pour contribuer à faciliter leur intégration dans la communauté nationale. [...] Il faut avant tout rechercher les solutions là où Suisses et étrangers partagent leur existence sur le plan humain, culturel, social et économique, c'est-à-dire dans les entreprises, sur les lieux de travail, au lieu de domicile et dans les quartiers, au sein des sociétés, et aussi dans les communes et les paroisses, sans oublier les écoles.

Dans cette phase, l'encouragement et la promotion de l'intégration deviendront un des éléments essentiels de la politique en matière de population étrangère, avec l'objectif d'améliorer

64. Ibid., p. 1368.

65. Ibid., p. 1369. 
le statut de celle-ci. Le 20 octobre 1977 sera par ailleurs déposée l'initiative populaire "Être solidaires", promue par la gauche et les syndicats, qui tend à l'abolition du statut de travailleur saisonnier et à la stabilisation des effectifs des travailleuses et travailleurs étrangers. Une fois admis en Suisse, ceux-ci doivent être considérés comme les égaux des Suisses, sauf en ce qui concerne la jouissance des droits politiques. L'initiative est toutefois largement refusée, par $84 \%$ des voix exprimées, le 5 avril 1981, probablement en raison de la crainte, commune à la population et au Gouvernement, d'une menace représentée par la présence étrangère pour divers secteurs économiques ${ }^{66}$. L'année d'après, le 6 juin 1982, la nouvelle loi sur les étrangers adoptée par les Chambres fédérales en date du 19 juin 1981 et conçue comme un contre-projet indirect à l'initiative "Être solidaires» sera aussi rejetée, en référendum populaire ${ }^{67}$. En décembre 1983 sera en outre rejeté un projet de modification de la Constitution fédérale destiné à faciliter la naturalisation des jeunes étrangères et étrangers nés en Suisse ou y ayant grandi et fréquenté l'école. Dans le message du Conseil fédéral, la naturalisation est présentée comme l'issue finale d'un processus d'intégration. On peut ainsi y lire ${ }^{68}$ :

Faciliter l'intégration dans la communauté suisse des étrangers vivant sur notre sol pendant une longue période correspond à un principe de la politique suisse envers les étrangers. Dans la mesure où ils sont intégrés socialement, économiquement et culturellement et approuvent nos institutions démocratiques, la possibilité doit leur être offerte d'acquérir la nationalité suisse à des conditions formelles et matérielles équitables. L'acquisition

66. Sur l'initiative, cf. Piguet, 2009, pp. 36-37.

67. Dans le texte de la nouvelle loi sur les étrangers, du 19 juin 1981, le terme intégration faisait son apparition, à l'art. 44, à propos des charges contributives des cantons: "Les cantons peuvent astreindre les employeurs qui ont des étrangers à leur service à participer aux frais des mesures sociales et d'intégration; en fixant le montant de la contribution, on tiendra compte des mesures prises par les entreprises elles-mêmes.» Voir, pour le texte de la loi, FF 1981 II 553-578, spéc. 565, pour l'art. 44, lequel ne figurait pas dans le projet de loi du Conseil fédéral, cf. Message à l'appui d’un projet de loi sur les étrangers, du 19 juin 1978, FF 1978 II 165-263, spéc. 248.

68. Message sur la révision du droit de la nationalité dans la Constitution fédérale, du 7 avril 1982, FF 1982 II 137-169, spéc. 146. Il y avait en réalité deux projets de modifications constitutionnelles soumis au vote du peuple et des cantons le 4 décembre 1983, le premier, l'arrêté fédéral sur la révision du droit de la nationalité dans la constitution fédérale, du 24 juin 1983 (FF 1983 II 719-720), a été accepté, le second, l'arrêté fédéral tendant à faciliter certaines naturalisations, également du 24 juin 1983 (FF 1983 II 721), a été rejeté (cf. pour les résultats du vote, FF 1984 I 621-623). 
de la nationalité suisse constitue la dernière étape vers leur pleine intégration dans notre communauté politique.

Au milieu des années 1980, après l'échec de l'initiative «Être solidaires» et un certain ralentissement de la menace xénophobe, on constate une nouvelle ouverture, limitée, à la main-d'œuvre étrangère. La Suisse enregistre toutefois encore une augmentation de l'immigration, égale à celle des années 1950 et 1960. Dans ce contexte, une énième initiative populaire "pour la limitation de l'immigration" lancée en 1985 sera encore rejetée le 4 décembre $1988^{69}$.

Les exigences de protection humanitaire et sociale sont donc reconnues par la politique, qui tend à atténuer ou à réfréner les pressions populistes visant à l'exclusion.

\subsubsection{0-2015: L'INTÉGRATION COMME TÂCHE DE L'ÉTAT ET DEVOIR DE LA PERSONNE ÉTRANGÈRE, OU ENCOURAGER ET EXIGER}

Depuis les années 1990, il est question de nouvelle politique en matière de migrations: la Suisse est confrontée aux exigences liées aux mouvements migratoires planétaires, à la libre circulation des personnes, aux nouveaux flux provenant de l'Europe de l'Est, aux besoins de main-d'œuvre étrangère liés au recul démographique. Entre 1987 et 1997, le Gouvernement présentera cinq rapports officiels d'experts sur le thème de la migration et de l'immigration, parmi lesquels le Rapport du Conseil fédéral sur la politique à l'égard des étrangers et des réfugiés, du 15 mai $1991^{70}$. Le Conseil fédéral y souligne la dimension planétaire du phénomène migratoire et fait le point sur la politique qu'il a suivie jusqu'alors en la matière. $\mathrm{Si}$, durant la période précédant la Première Guerre mondiale, cette politique tendait à la limitation de la libre circulation des personnes, destinée à contrôler le marché du travail et à préserver l'identité nationale ${ }^{71}$, dans la phase actuelle, l'objectif est de "créer des conditions favorables à l'intégration des travailleurs et résidants étrangers ${ }^{72}$. Cet objectif nécessite "la mise en œuvre d'une politique d'intégration à grande échelle. Une telle politique n'est efficace que lorsque Suisses et étrangers font un effort de

69. Voir, pour le résultat de la votation populaire, FF 1989 I 226-229.

70. FF 1991 III 316-348.

71. Voir Mahon et Collette, 2011, pp. 232-233.

72. FF 1991 III 316-348, p. 321. 
compréhension réciproque et elle dépend donc beaucoup du niveau d'information des deux parties " ${ }^{73}$. Dans les années 1990, le thème ne sera donc plus l'assimilation des personnes étrangères, mais bien plus, timidement, celui de leur intégration, selon une logique binaire: promouvoir la capacité des personnes étrangères à s'intégrer et obliger les autorités à mettre en œuvre une vaste politique d'intégration, qui ne pourra réussir que si populations suisse et étrangère savent faire preuve de compréhension réciproque. L'objectif de l'intégration mentionné dans le rapport du Conseil fédéral comme second pilier de la politique à l'égard des étrangers pourra apparaître motivé d'autant plus par la prévision de l'arrivée de groupes de population culturellement plus éloignés que les groupes issus des bassins de migration traditionnels ${ }^{74}$. C'est enfin dans son message relatif à la révision totale de la loi sur l'asile ainsi qu'à la modification de la loi sur le séjour et l'établissement des étrangers, du 4 décembre $1995^{75}$, que le Conseil fédéral affirmera de manière tout à fait explicite l'idée que l'intégration constitue une tâche de l'État. Ce message résume les trois piliers de la politique migratoire et discute des articulations possibles des compétences en matière d'intégration entre communes et cantons $^{76}$ :

Depuis 1970, la politique du Conseil fédéral à l'égard des étrangers repose sur trois piliers. Les mesures prévues pour limiter les nouvelles entrées sont destinées à assurer une juste proportion entre la population suisse et la population résidente étrangère; la pratique en matière d'accès des étrangers au marché du travail doit améliorer la structure de ce dernier et équilibrer si possible le marché de l'emploi; enfin, la consolidation du statut des étrangers au fur et à mesure des années de séjour et d'autres mesures d'encouragement visent à parfaire leur intégration sociale. Conformément à l'article 16 LSEE, les autorités chargées de délivrer les permis doivent tenir compte des intérêts moraux et économiques du pays, et du degré de la surpopulation étrangère. La promotion de l'intégration, tâche de dimension politique nationale, doit désormais être inscrite également dans la loi.

73. Ibid.

74. Ibid., p. 347.

75. FF 1996 II 1-187.

76. Ibid., pp. 33-34. 
Le Conseil fédéral précise que la promotion de l'intégration doit aller bien au-delà des sphères sociales et d'assistance - comme c'était le cas jusqu'alors -, acquérant ainsi une dimension politique. Il affirme en outre qu'avec une politique d'intégration qualifiée de décidée et convaincante, il est possible de promouvoir la confiance des étrangères et étrangers dans les personnes, dans l'ordre juridique et dans les institutions du pays, réduisant du même coup le risque de mouvements radicalisés et fondamentalistes. La promotion de l'intégration de la part du Gouvernement suisse vise ainsi aussi à contenir la xénophobie et le racisme. Dans ce contexte, la notion d'intégration fait donc son apparition officielle avec l'adoption par le Parlement, le 26 juin 1998, des articles 25 al. 1, let. i, et $25 a$ de la loi sur le séjour et l'établissement des étrangers (LSEE), entrés en vigueur le $1^{\text {er }}$ octobre 1999. Avec l'approbation successive de la nouvelle loi fédérale sur les étrangers (LEtr), du 16 décembre 2005, la politique d'intégration s'inscrira comme un élément central du droit des étrangers. Cette loi fera place à l'idée d'une intégration dite duale, résumée par la formule «encourager et exiger" et explicitée dans l'ordonnance sur l'intégration des étrangers, du 24 octobre 2007 (OIE) ${ }^{77}$, dont le chapitre 2 est intitulé "Contribution et devoirs des étrangers». À l'intérieur de celui-ci, l'article 4 traite de la «Contribution des étrangers à l'intégration", introduisant par ailleurs, comme instrument, la convention d'intégration. Plus récemment, entre 2011 et 2015, tant la loi sur les étrangers que la loi sur la nationalité - et les divers critères d'intégration qu'elles contiennent - ont fait l'objet de discussions et de propositions et projets de révisions. Ainsi, dans son message concernant la révision totale de la loi sur l'acquisition et la perte de la nationalité suisse (loi sur la nationalité, LN), du 4 mars 2011, le Conseil fédéral a soulevé la question d'une harmonisation de la notion d'intégration contenue dans la loi sur la nationalité avec celle prévue par la loi sur les étrangers, à la suite de l'entrée en vigueur de celle-ci et des diverses modifications subies, au cours des années, par la loi sur la nationalité elle-même. Les objectifs principaux annoncés dans ce projet de réforme étaient les suivants ${ }^{78}$ :

- assurer une large cohérence avec la loi sur les étrangers en ce qui concerne les exigences posées aux étrangers en matière d'intégration et de connaissances linguistiques;

77. RS 142.205 .

78. FF 2011 2639-2698, en particulier F 2640. 
- améliorer les instruments de décision [...] afin de garantir que seuls les étrangers bien intégrés obtiennent la nationalité suisse;

- harmoniser les exigences cantonales et communales relatives aux délais de résidence $[\ldots]$;

- réduire les charges administratives des autorités cantonales et fédérales en simplifiant et en harmonisant les procédures, de même qu'en clarifiant leurs rôles respectifs en matière de naturalisation.

Lors de la discussion du projet de réforme, il apparaît qu'il existe, dans l'ordre juridique, des notions d'intégration presque identiques en matière de droit des étrangers et de droit de la naturalisation, ce qui, de l'avis même du Conseil fédéral, est «loin de faciliter la compréhension", voire "prête à confusion", de sorte qu'un effort de clarification est nécessaire ${ }^{79}$ :

En substance, la formulation proposée correspond dans une large mesure à ce que prévoit le droit en vigueur. Cependant, la notion d'intégration est maintenant harmonisée avec le droit des étrangers. Pour l'essentiel, elle repose sur le respect de la sécurité et de l'ordre publics, le respect des valeurs de la [Constitution], l'aptitude à communiquer dans l'une des langues nationales, ainsi que la volonté de participer à la vie économique et à acquérir une formation. Ce dernier élément renvoie à l'art. 4, let. d, de l'ordonnance du 24 octobre 2007 sur l'intégration des étrangers (OIE; RS 142.205). Bien que la liste des critères d'intégration figurant aux art. 12 et 20 de la loi clarifie les conditions de naturalisation, il sera nécessaire de préciser dans la future ordonnance d'exécution les seuils d'une intégration suffisante, en tenant compte du développement du droit suisse de l'intégration.

Enfin, dans son message du 8 mars 2013 concernant la modification de la loi fédérale sur les étrangers (intégration), le Conseil fédéral proposait encore une modification de ladite loi dans le sens d'une rigidité accrue des mesures - et des exigences - d'intégration, propres à mettre en valeur, d'une part, «le potentiel des habitants [...] en tenant compte de leur diversité» et, d'autre part, à en appeler à la «responsabilité individuelle des étrangers en matière d'intégration». Il entend consolider aussi et ainsi «le caractère

79. Ibid., pp. 2645-2646. 
contraignant et réciproque de la politique d'intégration suisse». Ce durcissement de la ligne politique est confirmé par la proposition de vérifier le processus d'intégration aussi pour l'octroi de permis d'établissement ${ }^{80}$. En juin 2014 , le projet de révision totale de la loi sur la nationalité a été approuvé - non sans avoir été longuement discuté, amendé et durci - par les Chambres fédérales. La loi, adoptée le 20 juin 2014, a introduit le concept d'intégration réussie et définit de manière plus précise et exigeante les critères d'une telle intégration en vue de l'acquisition de la nationalité suisse $^{81}$. Le Conseil fédéral a adopté l'ordonnance d'application de la nouvelle loi en juin $2016^{82}$ et celle-ci, avec cette ordonnance, est entrée en vigueur le $1^{\mathrm{er}}$ janvier 2018. On présentera brièvement, plus loin, les principales modifications qu'elle apporte, tant sur le fond que du point de vue procédural.

On mentionnera encore que, dans l'intervalle, en 2015, à la suite de l'acceptation par le peuple et les cantons, le 9 février 2014, de l'initiative populaire "contre l'immigration de masse», le Parlement a demandé au Conseil fédéral de retravailler le projet de modification de la loi sur les étrangers afin de l'adapter au nouvel article $121 \mathrm{a}$ de la Constitution fédérale issu de cette initiative. Par conséquent, deux projets ont finalement été soumis au Parlement, en décembre 2016, que l'Assemblée fédérale a tous deux acceptés, l'un concernant la mise en ouvre de l'initiative "contre l'immigration de masse ${ }^{83}$, l'autre concernant la question de l'intégration ${ }^{84}$. À partir des années 1990, l'institution de réinvention tend à devenir plus sophistiquée et complexe. Une politique de type purement assimilationniste ne pouvant plus se justifier et se légitimer, ni historiquement, ni socialement, l'intégration est conçue comme relevant d'une politique publique, impliquant des mesures à charge de la Confédération elle-même et des cantons. Demeurent naturellement prioritaires dans le processus d'intégration l'engagement des personnes étrangères et leur sens des responsabilités. L'intégration se

80. FF 2013 2131-2190, 2132 pour ces formulations.

81. Voir FF 2014 5001-5016, ainsi que RO 2016 2561-2576, pour le texte de la nouvelle loi.

82. Ordonnance sur la nationalité suisse (ordonnance sur la nationalité, $\mathrm{ON}$ ), du 17 juin 2016, RO 2016 2577-2592.

83. Loi fédérale sur les étrangers (LEtr) (Gestion de l'immigration et amélioration de la mise en ouvre des accords sur la libre circulation des personnes), Modification du 16 décembre 2016, FF 2016 8651-8658.

84. Loi fédérale sur les étrangers (LEtr) (Intégration), Modification du 16 décembre 2016, FF 2016 8633-8650. 
concrétisera donc, comme nous le verrons dans le chapitre suivant, en une série de critères auxquels la personne étrangère, candidate à la naturalisation notamment, devra répondre.

\subsection{SYNTHÈSE: DE L'INCORPORATION À L'INTÉGRATION COMME INSTITUTION DE RÉINVENTION}

L'analyse historico-juridique proposée des principales étapes politico-législatives de l'évolution de la réglementation de la présence et du statut des personnes étrangères sur le territoire suisse a permis de mettre en lumière la manière dont la notion d'intégration a progressivement fait son apparition dans l'ordre juridique suisse et les diverses significations que cette notion a assumées au cours du temps dans les débats politiques et dans les réglementations normatives.

Plus spécifiquement, en parcourant quelques-unes des principales étapes historiques et politico-législatives, de 1848 à nos jours, nous avons cherché à montrer comment la réglementation du droit de séjour des personnes étrangères sur le territoire national a été conditionnée, à travers les différentes époques, par des facteurs historiques, démographiques et sociaux qui ont déterminé l'adoption de projets législatifs et de mesures d'orientation fort différentes. Ces mesures vont d'une sorte de naturalisation forcée, destinée à équilibrer la proportion entre population suisse et population étrangère, dans les années 1920, jusqu'à l'introduction d'une véritable politique et de mesures d'intégration, dans les années 1970, en passant notamment par l'instauration des permis de séjour pour les personnes étrangères, dans les années 1940, et la promulgation d'une loi sur l'acquisition et la perte de la nationalité suisse, dans les années 1950 (loi modifiée ensuite en 1990 et en 2014).

C'est ainsi qu'après une phase dite libérale de la politique fédérale et cantonale en matière de naturalisation, phase dans laquelle la présence étrangère a été gérée par les cantons au moyen de l'instrument de la naturalisation, ultérieurement, à la suite de la Première Guerre mondiale, avec l'affirmation des États-nations et l'accroissement du nombre des étrangères et des étrangers, la Confédération s'est progressivement arrogé - par la modification de la Constitution de 1848 - une série de compétences en matière d'octroi de la nationalité. Parmi ces compétences figure en premier lieu celle d'évaluer les liens de la personne étrangère avec son pays 
d'origine. À partir de cette phase déjà, malgré une aspiration cantonale à une politique libérale, la prérogative de la Confédération d'évaluer les exigences telles que la valeur morale des candidates et des candidats, en plus de leurs liens avec le pays d'origine, crée les prémisses qui permettront de développer ce que nous avons défini comme une institution de réinvention de la personne étrangère (1.4.1). Cela sera possible à travers l'introduction d'une série de mesures de nature politico-administrative, mais aussi de caractère social.

La Confédération a ainsi fait recours dans un premier temps à des traités d'établissement avec certains États voisins pour accueillir les étrangers sur son territoire. Par la suite, après une révision de la Constitution qui lui en a donné la compétence, en 1925, elle a adopté une véritable législation fédérale sur le séjour et l'établissement des étrangers (LSEE), en 1931, laquelle est restée en vigueur jusqu'à fin 2007. Début 2008 est entrée en vigueur la LEtr, nouvelle loi fédérale sur les étrangers, devenue aujourd'hui loi sur les étrangers et l'intégration (LEI). De cette manière, un dispositif central de contrôle et de réglementation de la politique relative à la population a permis de gérer la présence des étrangères et des étrangers, non seulement à travers l'instrument de la naturalisation forcée - envisagé dans les années 1920 -, mais aussi à travers la mise en place d'une politique des permis et, par la suite, de quotas, destinée à contrôler ou à limiter leur séjour.

C'est dans cette phase que l'institution de réinvention commence à prendre une forme plus définie. La naturalisation est vue comme un instrument de gestion du social. Rééquilibrer les rapports entre population étrangère et population nationale est jugé nécessaire et possible non seulement à travers l'adoption de mesures législatives et administratives (naturalisations et permis de séjour), mais également à travers l'implication active de la population locale, appelée à participer à l'entreprise quotidienne de transformation identitaire des étrangères et des étrangers.

En termes de mesures législatives adoptées dans un paysage sociopolitique et démographique en mutation, à la suite des deux guerres mondiales, la nouvelle loi fédérale de 1952 sur l'acquisition et la perte de la nationalité suisse (loi sur la nationalité, LN), se substituant à une ancienne loi de 1903 et rassemblant dans le droit ordinaire plusieurs réglementations auparavant dispersées, avait ainsi pour but de créer un véritable code de la nationalité. À partir 
de ce moment, dans la seconde moitié du $\mathrm{XX}^{\mathrm{e}}$ siècle, bien que reste inchangé, pour la naturalisation, le critère de l'aptitude de la personne étrangère à devenir suisse (art. 14 let. a LN), celle-ci tend aussi à être prise en considération comme personne titulaire de droits, et non plus seulement comme destinataire passive d'un processus d'assimilation.

Jusqu’à la fin des années 1970, la politique d'accueil des étrangères et étrangers a été modulée en fonction des besoins de l'économie et de la population suisses, oscillant entre deux pôles: un marché du travail flexible et une politique de naturalisation restrictive. Elle a paru s'adoucir seulement à partir des années 1980.

La notion d'intégration a fait, comme nous l'avons vu, sa première apparition officielle vers la moitié des années 1970. Les mesures d'intégration introduites durant les années 1970 ont d'abord été destinées à favoriser l'intégration de la main-d'œuvre immigrée, avant de devenir, à partir des années 1980, l'objet d'une véritable politique publique d'intégration, considérée comme une tâche de l'État et acquérant une dimension hautement politique, fédérale et non plus seulement cantonale, tendant à garantir la coexistence et la tolérance réciproque entre populations étrangère et suisse.

La notion d'intégration a ainsi assumé des connotations différentes et variées. Tout d'abord, par son insertion dans la loi sur la nationalité, au début des années 1990, en tant que condition ou exigence pour obtenir la naturalisation; par la suite, dans les années 2000, dans la loi sur les étrangers (LEtr), en tant que critère pour la concession de permis et en vue de réglementer la coexistence quotidienne entre étrangères et étrangers et Suisses. Avec la nouvelle loi fédérale sur les étrangers, de 2005, entrée en vigueur en 2008, l'intégration est conçue, comme l'exprime l'art. 4, comme un processus mutuel et réciproque, fondé sur la disponibilité des étrangers à s'intégrer dans le contexte social du pays et sur l'accueil que leur réserve la communauté suisse.

Le processus de révision et d'harmonisation du droit de la migration s'est conclu en juin 2014, avec l'adoption d'une nouvelle loi fédérale sur la nationalité. Celle-ci a introduit le concept d'intégration réussie comme condition pour les candidates et les candidats à la naturalisation (art. 11 let. a de la nouvelle LN). Elle a aussi substitué à l'exigence de s'être «accoutumé au mode de vie et aux usages suisses» (art. 14 let. b LN), contenue dans la 
version révisée en 1990 de l'ancienne loi sur la nationalité de 1952 , en vigueur jusqu'au 31 décembre 2017, celle de s'être "familiarisé avec les conditions de vie en Suisse» (art. 11 let. b nouvelle LN) ${ }^{85}$. On a procédé, par la même occasion, à une redéfinition des critères et des conditions dites matérielles d'acquisition de la naturalisation et, donc, de la notion d'intégration, ancrée entre autres dans cette idée de la familiarisation avec les conditions de vie en Suisse (art. 11 et $12 \mathrm{LN}$ ). De son côté, le projet de réforme de la loi sur les étrangers de 2013 a prévu un renforcement des mesures d'intégration pour les personnes qui sollicitent un permis de séjour, renforcement qui, dans l'esprit du législateur, est propre à protéger, d'une part, le "potentiel de la population résidente» et à raffermir, d'autre part, «le sens de la responsabilité des étrangers", de manière à consolider le caractère obligatoire du processus d'intégration ${ }^{86}$.

85. Il faut souligner que le verbe "accoutumer" est depuis toujours traduit par «se familiariser » dans la version italienne de la loi.

86. Dans l'intervalle, en 2015 , la loi sur les étrangers a fait l'objet de nouvelles procédures de révision, à la suite de l'acceptation par le peuple et les cantons, le 9 février 2014, de l'initiative populaire "contre l'immigration de masse». 


\title{
4. DÉFINIR L'INTÉGRATION: SIGNIFICATIONS LÉGALES ACTUELLES
}

\author{
FLORA DI DONATO ET PASCAL MAHON
}

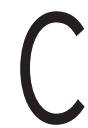

omme on l'a vu dans le chapitre précédent ${ }^{1}$, la notion d'intégration est apparue progressivement dans l'ordre juridique suisse et elle s'est installée - même fortement - dans plusieurs ensembles législatifs. Elle figure dans deux législations au moins au niveau fédéral: la loi fédérale sur l'acquisition et la perte de la nationalité suisse, dans sa version ancienne du 29 septembre 1952 (en vigueur jusqu'au 31 décembre 2017) et dans sa version nouvelle, entièrement révisée, du 20 juin 2014 (en vigueur depuis le $1^{\text {er }}$ janvier 2018), d'une part; la loi fédérale sur les étrangers, du 16 décembre 2005, d'autre part. La notion figure aussi dans divers actes législatifs cantonaux, comme la loi sur l'intégration et la cohésion multiculturelle du canton de Neuchâtel, du 26 août 1996.

Bien que traitant de deux situations différentes, ces réglementations font toutes deux de la notion d'intégration un élément central du régime juridique qu'elles mettent en place.

Le but de ce chapitre est donc d'expliciter la notion d'intégration telle qu'elle est conçue et perçue aujourd'hui, en Suisse, du côté des autorités politiques, administratives et judiciaires, et cela aussi bien sous l'angle de la législation relative à l'acquisition de la nationalité que sous celui de la législation relative au droit de séjour des étrangères et des étrangers. Il a pour objet de reconstruire la ou les significations de la notion d'intégration de manière synchronique, et non plus diachronique comme dans le chapitre précédent.

Nous verrons donc en particulier de quelle manière ce que nous avons défini, dans le chapitre premier, puis dans le chapitre 3, comme une institution de réinvention prend concrètement forme à

1. Comme le précédent, ce chapitre propose aussi une version synthétique et retravaillée de l'étude de Di Donato, 2016, qui constituait elle-même le premier résultat concret du projet de recherche dont le présent ouvrage est l'aboutissement. 
travers des mesures et des critères destinés à homologuer, en quelque sorte, la conduite de la personne candidate à la naturalisation en la comparant à celle de la population suisse. Nous verrons aussi si et dans quelle mesure ces critères sont également applicables aux étrangères et aux étrangers qui aspirent non pas à la naturalisation mais à un (simple) titre de séjour.

Dans un premier temps, nous reviendrons brièvement sur la notion d'intégration dans plusieurs législations, afin d'expliciter la problématique qui en résulte (4.1). Nous chercherons à analyser ensuite le sens de la notion d'intégration dans le droit positif suisse, d'abord dans le droit de la nationalité et plus particulièrement de la naturalisation, où il s'agira de se pencher sur les critères de l'aptitude à devenir suisse, et de l'intégration que celle-ci implique, tout en prenant en considération la pratique interprétative fédérale et jurisprudentielle en matière d'acquisition de la nationalité (4.2). Un examen analogue sera ensuite proposé pour ce qui est du droit relatif au séjour et à l'établissement des étrangères et des étrangers (4.3). Une brève analyse de la loi du canton de Neuchâtel sur l'intégration et la cohésion multiculturelle, qui s'inspire des principes d' "égale dignité» et de "coexistence pacifique», permettra de montrer comment la législation fédérale en matière d'intégration est susceptible de traductions différentes, au niveau local, et peut ainsi ouvrir la voie à un modèle réputé libéral de l'intégration (4.4). Enfin, nous proposerons une brève synthèse générale et conclusive (4.5).

\subsection{L'UBIQUITÉ DE LA NOTION D'INTÉGRATION}

Ainsi que nous l'avons vu, la notion d'intégration trouve aujourd'hui son point d'ancrage juridique et normatif essentiel dans deux législations clés, de rang fédéral: la loi sur la nationalité (LN) et la loi sur les étrangers (LEtr). Il s'agit là de deux lois produites à des époques différentes, qui règlent, respectivement, l'acquisition et la perte de la nationalité suisse, pour la première, et l'entrée, la sortie, le séjour et l'établissement ainsi que le regroupement familial des étrangères et des étrangers en Suisse, pour la seconde.

L'analyse parallèle de ces deux textes normatifs vise à répondre à la question de savoir si le degré d'intégration que l'on est en droit d'attendre d'une personne candidate à la naturalisation suisse est le même que celui que l'on est en droit d'attendre d'une personne renouvelant son titre de séjour. 
Autrement dit, ce chapitre explore si et, le cas échéant, pour quelles raisons la notion d'intégration, définie de manière presque similaire dans deux contextes législatifs différents, revêt ou doit revêtir des significations identiques ou, au contraire, différentes. Il semble en effet régner à cet égard une certaine ambiguïté dans les intentions du législateur. Une telle ambiguïté est en outre accentuée par le fait que, en dépit d'un cadre législatif uniforme au plan fédéral, l'ordre juridique suisse délègue aux cantons la politique d'intégration, leur permettant de proposer en cette matière des mesures publiques diversifiées. Il s'agira donc de vérifier ce qu'il en est effectivement, au travers de la pratique des autorités administratives et de la jurisprudence des tribunaux.

\subsection{LA NOTION D'INTÉGRATION DANS LA LOI FÉDÉRALE \\ SUR LA NATIONALITÉ (LN)}

Le premier ensemble législatif au sein duquel est formulé le critère de l'intégration en tant qu'exigence pour l'obtention de la citoyenneté est celui de la loi sur la nationalité, adoptée dans les années 1950 et successivement modifiée dans les années 1990, puis entièrement révisée en 2014.

Nous examinerons ici, dans un premier temps, comment le critère de l'intégration a fait son apparition, en 1990, dans la loi sur la nationalité de 1952, et quels ont été les objectifs et la signification de ce changement (4.2.1). Nous nous pencherons ensuite sur la révision totale de la loi, du 20 juin 2014, et l'introduction, dans ce cadre, du concept d'intégration réussie (4.2.2), pour analyser, en troisième lieu, la notion d'intégration dans la pratique administrative et la jurisprudence fédérales (4.2.3). Un bref excursus sera consacré à la question de la protection judiciaire en la matière (4.2.4), avant que ne soient proposées quelques conclusions intermédiaires (4.2.5).

\subsubsection{2-1990: LA RÉVISION DE L'ARTICLE 14 LN}

\section{ET L'INTRODUCTION DU CRITÈRE DE L'INTÉGRATION}

Comme nous l'avons vu au chapitre précédent, dans un contexte sociopolitique qui a changé à la suite de la Seconde Guerre mondiale, la nationalité est présentée comme une valeur tant pour l'individu que pour l'État, lequel doit sauvegarder l'intérêt de la communauté 2 .

2. Voir le Message du Conseil fédéral à l'Assemblée fédérale relatif à un projet de loi sur l'acquisition et la perte de la nationalité suisse, du 9 août 1951, FF 1951 II 665-718. 
L'exigence de l'aptitude de la personne étrangère à l'acquisition de la nationalité est posée comme la base du processus de naturalisation, processus qui consiste en l'élection d'un individu jugé apte à devenir citoyen ${ }^{3}$ :

Seul l'étranger apte à devenir Suisse et digne de l'être doit être naturalisé. Il faut qu'il se soit adapté d'une manière décisive aux conditions suisses, qu'il se soit assimilé. Il faut qu'en considération de son genre de vie, de son caractère, de toute sa personnalité, on puisse admettre qu'il fera certainement un bon Suisse, digne de toute confiance. En un mot, la naturalisation est l' "élection» d'un homme d'après son aptitude à devenir citoyen.

Dans cette première formulation législative, le critère de l'aptitude introduit à l'article 14 de la nouvelle loi sur la nationalité présente toutefois encore des contours indéfinis ${ }^{4}$ :
Art. 14 Enquête
${ }^{1}$ Avant l'octroi de l'autorisation, l'aptitude du requérant à la natu- ralisation doit être examinée.
${ }^{2}$ L'enquête doit donner une image aussi complète que possible de la personnalité du requérant et des membres de sa famille.

Le mécanisme de transformation de la personne étrangère se décline ici à travers l'introduction de l'exigence de l'aptitude à devenir suisse, qui requiert sans trop de moyens termes une assimilation - du caractère, des habitudes, du style de vie et de la personnalité de l'étrangère ou de l'étranger - aux us et coutumes suisses. L'étrangère ou l'étranger doit devenir une personne de confiance aux yeux de ses futurs concitoyennes et concitoyens.

Par la suite, jugé quelque peu désuet et susceptible d'interprétations différentes, et parfois discriminatoires, dans l'appréciation du profil des candidates et candidats, le concept a été modifié. Il a ainsi été décidé, avec la révision partielle de l'article 14 au début des années 1990, qu'il y aurait lieu, avant de concéder l'autorisation fédérale de naturalisation, que l'autorité examine l'aptitude à devenir suisse, par la vérification de l'intégration et la familiarisation

3. Ibid., p. 676.

4. Voir le texte de la Loi fédérale sur l'acquisition et la perte de la nationalité suisse, du 29 septembre 1952, FF 1952 III 137-151. 
avec les us et coutumes suisses, en plus de la vérification de la conformité à l'ordre juridique suisse et le fait de ne pas compromettre la sûreté intérieure ou extérieure du pays. L'article $14 \mathrm{LN}$ a donc été reformulé ainsi ${ }^{5}$ :
Art. 14 Aptitude
Avant l'octroi de l'autorisation, on s'assurera de l'aptitude du requérant à la naturalisation. On examinera en particulier si le requérant:
a. S'est intégré dans la communauté suisse;
b. S'est accoutumé au mode de vie et aux usages suisses;
c. Se conforme à l'ordre juridique suisse; et
d. Ne compromet pas la sûreté intérieure ou extérieure de la Suisse.

Dans le cadre du critère de l'aptitude, l'article 14 dans sa nouvelle formulation de 1990 introduit donc un nouvel élément, celui de l'intégration: la personne candidate doit s'être intégrée à la communauté suisse pour obtenir la naturalisation.

Dans cette phase, la volonté transformatoire de l'identité de la personne semble s'atténuer par le biais de l'introduction d'un autre critère, celui de l'intégration. Là encore, il s'agit d'un critère qui n'est pas défini de manière explicite par la loi - pas plus que le critère de l'aptitude - et qui est dès lors susceptible d'interprétations administratives, jurisprudentielles et doctrinales non univoques. Toutefois, selon le Conseil fédéral, une telle absence de définition précise est voulue, du moment que la perception sociale de ce qui constitue l'intégration est susceptible de se modifier dans le temps. Il n'est donc pas possible de fixer a priori des critères législatifs abstraits qui soient définitifs ${ }^{6}$.

Il n'en demeure pas moins que le Conseil fédéral énonce quelques éléments qui ont trait par exemple à l'autonomie financière, à la participation à la vie publique et sociale, ainsi qu'à la bonne réputation de la personne candidate ${ }^{7}$ :

Une intégration réussie dépend non seulement d'une bonne réputation (voir aussi let. c) et de l'aptitude du candidat à communiquer

5. Voir la Loi sur la nationalité, selon modification du 23 mars 1990, FF 1990 I 1519-1528.

6. Voir le Message concernant le droit de la nationalité des jeunes étrangers et révision de la loi sur la nationalité, du 21 novembre 2001, FF 2002 1815-1899, spéc. 1844.

7. Ibid. 
avec l'entourage (let. b), mais se traduit également par la capacité de mener une vie autonome, par l'intérêt et la participation à la vie publique et sociale (p. ex. dans les domaines culturel et sportif; participation à des manifestations de quartier ou villageoises, etc.), sans oublier le délai fédéral de résidence (voir art. 15), en tant que critère d'intégration purement objectif.

L'accent semble se déplacer ici de l'évaluation de la personnalité de la candidate ou du candidat à la naturalisation à ses attitudes dans la vie quotidienne: capacité de communiquer dans une langue nationale, capacité d'autonomie sur le plan professionnel et capacité d'insertion sociale. Dans son message concernant la modification de la loi sur la nationalité du 26 août 1987, le Conseil fédéral explique en outre que le terme intégration désigne " [... l'accueil des étrangers dans la communauté suisse et la disposition des étrangers à s'insérer dans le milieu social suisse, sans qu'ils soient pour autant obligés de renoncer à leurs liens culturels et à leur nationalité d'origine»; le Gouvernement met le concept d'intégration en relation avec le «fait de s'accoutumer ${ }^{8}$ :

Le fait de s'accoutumer (let. b) est une conséquence de l'intégration et désigne l'adoption du mode de vie et des usages suisses par l'étranger [...]. Le terme "s'accoutumer» est préférable à celui de "s'assimiler», étant donné que celui-là, contrairement à celui-ci, ne véhicule aucune connotation négative. $\mathrm{Nul}$ n'exige du candidat à la naturalisation qu'il renonce à l'identité qu'il avait jusque là pour "changer de peau». S'accoutumer veut dire pour l'étranger parvenir à l'issue d'une phase décisive du processus qui aboutira au mariage de ses deux cultures, suisse et étrangère. Ce critère, ainsi que celui de l'intégration, est aussi mentionné expressément dans les législations cantonales sur la naturalisation.

Le Conseil fédéral spécifie enfin les critères prévus par les lettres c et d, c'est-à-dire le fait de se conformer à l'ordre juridique suisse et de ne pas compromettre la sûreté intérieure ou extérieure de la Suisse ${ }^{9}$ :

Pour obtenir l'autorisation fédérale de naturalisation, le requérant doit par ailleurs se conformer à l'ordre juridique suisse (let. c). Il

8. FF 1987 III 285-336, spéc. 296.

9. Ibid., 296-297. 
doit avoir bonne réputation en matière pénale et en matière de poursuites et faillites. [...] On peut en effet exiger d'un candidat à la naturalisation qu'il reconnaisse les institutions démocratiques de notre pays.

La sûreté intérieure ou extérieure de la Suisse (let. d) est un terme qui est aussi utilisé dans d'autres domaines du droit. Ainsi, l'article 70 de la constitution prévoit que la Confédération a le droit de renvoyer de son territoire les étrangers qui compromettent la sûreté intérieure ou extérieure de la Suisse.

D'autres conditions de naturalisation, en plus de celles énumérées, peuvent être imposées par le droit cantonal.

\subsubsection{LE CONCEPT D'INTÉGRATION RÉUSSIE}

\section{DANS LA LOI SUR LA NATIONALITÉ DU 20 JUIN 2014}

Entre 2011 et 2015, tant la loi sur les étrangers que la loi sur la nationalité ont fait l'objet de discussions et de projets de révision. Dans son message concernant la révision totale de la loi sur la nationalité suisse (loi sur la nationalité, LN), du 4 mars 2011, le Conseil fédéral a soulevé la question d'une harmonisation de la notion d'intégration qui se trouve contenue dans les deux ensembles législatifs, à la suite de l'entrée en vigueur de la nouvelle loi sur les étrangers, en 2008, et des diverses modifications de la loi sur la nationalité. Dans la discussion du projet de réforme, il est fait allusion à l'existence de deux notions presque identiques, ce qui, selon le Conseil fédéral, «loin de faciliter la compréhension [...], prête à confusion", de sorte qu'il "importe donc de bien définir la terminologie utilisée ${ }^{10}$. Au paragraphe 1.2.2.1 du message, intitulé "Notion d'intégration", il est pris acte de la définition de l'intégration contenue dans la loi sur la nationalité et de celle comprise dans la loi sur les étrangers ${ }^{11}$ :

Pour l'essentiel, elle repose sur le respect de la sécurité et de l'ordre publics, le respect des valeurs de la Cst., l'aptitude à communiquer dans l'une des langues nationales, ainsi que la volonté de participer à la vie économique et à acquérir une formation. Ce dernier élément renvoie à l'art. 4, let. d, de l'ordonnance du 24 octobre 2007 sur l'intégration des étrangers (OIE; RS 142.205).

10. FF 2011 2639-2698, spéc. 2645.

11. Ibid. 
La ratio du projet de réforme est donc d'harmoniser ces notions tant au niveau vertical (en relation avec les exigences des cantons et des communes) qu'horizontal (par rapport au droit des étrangers) ${ }^{12}$.

D'autre part, ainsi que nous l'avons déjà indiqué (3.2.6), le projet du Conseil fédéral de révision de la loi sur les étrangers du 8 mars 2013 proposait un renforcement des mesures et des exigences d'intégration dans cette loi, de manière à mettre en valeur, d'une part, "le potentiel des habitants [...] en tenant compte de leur diversité» et, d'autre part, à en appeler à la «responsabilité individuelle des étrangers en matière d'intégration". Quant à la révision totale de la loi sur la nationalité adoptée le 20 juin 2014, elle donne une définition plus précise avec le concept d'intégration réussie. Elle détaille les critères de cette intégration dans les articles 11 et 12 de la loi, qui déterminent les conditions matérielles d'obtention de la naturalisation et les critères d'intégration:

\section{Art. 11 Conditions matérielles}

L'autorisation fédérale de naturalisation est octroyée si le requérant remplit les conditions suivantes:

son intégration est réussie;

il s'est familiarisé avec les conditions de vie en Suisse;

il ne met pas en danger la sûreté intérieure ou extérieure de la Suisse. Art. 12 Critères d'intégration

${ }^{1}$ Une intégration réussie se manifeste en particulier par:

a. le respect de la sécurité et de l'ordre publics;

b. le respect des valeurs de la Constitution;

c. l'aptitude à communiquer au quotidien dans une langue nationale, à l'oral et à l'écrit;

d. la participation à la vie économique ou l'acquisition d'une formation; et

e. l'encouragement et le soutien de l'intégration du conjoint, du partenaire enregistré ou des enfants mineurs sur lesquels est exercée l'autorité parentale.

${ }^{2} \mathrm{La}$ situation des personnes qui, du fait d'un handicap ou d'une maladie ou pour d'autres raisons personnelles majeures, ne remplissent pas ou remplissent difficilement les critères d'intégration prévus à l'al. 1, let. c et d, est prise en compte de manière appropriée. ${ }^{3}$ Les cantons peuvent prévoir d'autres critères d'intégration.

12. Voir Gutzwiller, 2015, p. 1. 
Parmi ces conditions matérielles, il est prévu que la personne requérante se soit intégrée avec succès et qu'elle se soit familiarisée avec les conditions de vie en Suisse. La nouvelle loi attribue un rôle central à l'intégration, dont elle fait le point d'arrivée du parcours dans la société suisse. Elle opère ainsi un "changement de paradigme» par rapport à la loi actuelle: l'intégration est requise avant l'octroi de la naturalisation, et non après ${ }^{13}$. En outre, sur le plan linguistique, on assiste à des modifications importantes: le critère de s'être "accoutumé au mode de vie et aux usages suisses" est remplacé par celui de s'être «familiarisé avec les conditions de vie en Suisse" (art. 11 let. b LN) ${ }^{14}$. L'encouragement et le soutien de l'intégration s'étendent aux conjointes et conjoints ainsi qu'aux partenaires de fait et aux mineurs sur lesquels la personne requérante exerce l'autorité parentale. Cela représente une réelle innovation en comparaison avec la loi précédente, du fait que le processus d'intégration ne concerne pas seulement la personne candidate à la naturalisation, mais aussi les membres de sa famille. La loi prévoit aussi un renforcement des exigences d'ordre linguistique, qui se traduit par le critère de l'" aptitude à communiquer au quotidien dans une langue nationale, à l'oral et à l'écrit" (art. 12 let. c) ${ }^{15}$. Enfin, les conditions de résidence sont réduites à dix ans à partir de la possession du permis de séjour (art. 9 al. 1 let. a), au lieu des douze années de l'ancienne loi ${ }^{16}$.

Dans l'actualité la plus récente, avec la réforme de 2014, en vigueur dès 2018, l'exigence de transformation de la personne réapparaît. S’il n'est certes plus possible de revenir au critère de l'assimilation, il n'est pas moins exigé que l'intégration soit réussie, critère qui est examiné avant la naturalisation. Celle-ci finit ainsi par devenir l'étape ultime du parcours d'intégration de la personne étrangère dans le pays d'accueil.

13. Voir Ibid., p. 6.

14. Selon le Rapport explicatif. Projet d'ordonnance sur la nationalité mis en consultation, août 2015 , p. 3, il s'agit là d'une modification purement terminologique (https://www.sem.admin. $\mathrm{ch} / \mathrm{dam} / \mathrm{data} / \mathrm{sem} /$ aktuell/gesetzgebung/buev/vn-ber-f.pdf, consulté le 16 janvier 2020).

15. Selon la nouvelle Ordonnance sur la nationalité suisse (ON), du 17 juin 2016 (RO 2016 2577-2592), le «requérant doit justifier de connaissances orales d'une langue nationale équivalant au moins au niveau B1 du Cadre européen commun de référence pour les langues (CECR) et de compétences écrites du niveau A2 au minimum» (art. 6 al. 1). Le Rapport explicatif (p. 9) explique qu'il s'agit de « connaissances linguistiques suffisantes pour pouvoir, en principe, faire face à la majorité des situations de la vie quotidienne, que ce soit sur leur lieu de domicile, sur leur lieu de travail ou dans l'espace public».

16. Il faut préciser toutefois que la nouvelle loi exige un permis d'établissement, ce qui n'est pas le cas dans le droit antérieur, où tous les types de permis sont admissibles. 


\subsubsection{LES CRITÈRES DE LA NATURALISATION DANS LA PRATIQUE}

\section{ET LA JURISPRUDENCE FÉDÉRALES}

Bien qu'il n'existe pas de définition uniforme du terme d'intégration et que le processus d'harmonisation entre la loi sur la nationalité et la loi sur les étrangers ne soit pas complètement abouti, la pratique et la jurisprudence fédérales contribuent à donner des significations à ce concept. En ce qui concerne la pratique, aujourd'hui, c'est le Manuel sur la nationalité proposé par le Secrétariat d'État aux migrations (SEM, ci-après: Manuel ou Manuel du SEM) qui spécifie comme suit les critères d'intégration applicables aux personnes candidates à la naturalisation ${ }^{17}$ :

- respecter les principes fondamentaux de la Constitution suisse;

- se conformer à l'ordre juridique suisse (et, par analogie, à l'ordre juridique étranger) [...];

- les cantons peuvent exiger que le requérant soit en mesure de subvenir à ses besoins de manière autonome et durable (pas de dépendance de l'aide sociale);

- participe[r] à la vie sociale;

- posséder des connaissances linguistiques suffisantes;

- entretenir des contacts avec la population;

- être professionnellement intégré.

En sus des critères ainsi mentionnés, le Manuel suggère une évaluation globale des exigences d'intégration, qui tienne compte de la situation individuelle de chaque personne et prenne en considération des facteurs comme l'âge, la formation, les éventuels handicaps, etc., comme nous le détaillons dans les chapitres 6 et 7 .

La première des exigences posées par le Manuel, celle du respect des principes fondamentaux de la Constitution fédérale, est spécifiée comme l'interdiction des comportements contraires aux principes constitutionnels, comme l'intolérance à l'égard d'autres communautés ou les formes d'extrémisme religieux. De même, des actes de violence ou des comportements répréhensibles sont considérés comme des signes d'une intégration insuffisante.

17. Voir le Manuel sur la nationalité, mis à jour en février 2015, qui, à son chapitre IV, définit les "Conditions générales et critères de naturalisation" (ch. 4.7.2.1). Ce manuel est publié sur le site du SEM, https:/www.sem.admin.ch/dam/data/sem/rechtsgrundlagen/ weisungen/buergerrecht/hb-bueg-kap1-f.pdf (consulté le 16 janvier 2020). 
Dans son message concernant la révision de la loi sur la nationalité, au paragraphe 1.2.2.4, intitulé Respect des valeurs de la Constitution, le Conseil fédéral propose, comme exemples de violation des «valeurs de la Cst. et des valeurs universelles de la protection internationale des droits de l'homme», le fait de rejeter "le monopole de la puissance publique ou l'égalité de l'homme et de la femme». Il précise cependant qu'un "comportement qui diverge de celui de la majorité de la population, mais qui est protégé par les droits fondamentaux est conforme aux valeurs de la [Constitution] et ne peut être interprété en défaveur du candidat à la naturalisation ${ }^{18}$. À côté du critère de l'intégration dans la communauté suisse prévu à l'art. 14 let. a LN, l'autre élément qui contribue à définir l'aptitude à la naturalisation est le fait de s'être "accoutumé au mode de vie et aux usages suisses» (art. 14 let. b LN). Le Secrétariat d'État aux migrations estime cette condition remplie «lorsque le requérant entretient des contacts réguliers avec la population suisse vivant dans sa localité ou s'engage en faveur d'une association ancrée à l'échelle locale» ${ }^{19}$. Selon le Manuel, en outre, cette familiarisation «se manifeste également à travers les connaissances civiques ou de la géographie et de l'histoire locales ${ }^{20}$. Le Conseil fédéral, à son tour, précise la notion de «se familiariser» en mettant l'accent, en plus de la fréquentation de la population locale et de la participation au monde associatif, sur la connaissance des droits politiques auxquels la naturalisation donne droit ${ }^{21}$ :

On peut parler de familiarité lorsqu'il a des contacts réguliers avec des citoyens suisses ou s'il participe aux activités d'une association locale. La familiarité avec les conditions locales s'exprime également à travers des connaissances sur les évènements historiques marquants et sur les particularités géographiques et politiques de la Suisse. Les personnes qui obtiennent la nationalité suisse accèdent également aux droits politiques et participent à la formation de la volonté politique dans notre pays.

18. FF 2011 2639-2698, spéc. 2647. Gutzwiller, 2015, p. 8, précise également que le respect des valeurs de la Constitution comprend non seulement les valeurs véhiculées par la charte constitutionnelle, mais aussi l'ensemble des valeurs liées à la protection internationale des droits humains.

19. Voir le Manuel, op. cit., spéc. p. 30.

20. Ibid.

21. FF 2011 2639-2698, spéc. 2649. 
De son côté, la jurisprudence a précisé que la participation à la vie associative locale n'est pas un élément déterminant à lui seul pour définir l'intégration, laquelle consiste en une assimilation progressive aux habitudes suisses ${ }^{22}$.

Dans son message concernant le droit de la nationalité des jeunes étrangères et étrangers et la révision de la loi sur la nationalité, du 21 novembre 2001, le Conseil fédéral avait précisé dans les termes suivants la différence entre être intégré, être accoutumé et être familiarisé ${ }^{23}$ :

La formulation actuelle de l'art. 14, let. a et b, en vertu duquel le candidat à la naturalisation doit être intégré dans la communauté suisse et accoutumé au mode de vie et aux usages suisses, est adaptée aux termes utilisés aujourd'hui. Ainsi, selon l'art. 14, let. $\mathrm{a}$ et $\mathrm{b}$, modifié, le candidat doit être intégré en Suisse et s'être familiarisé avec les conditions d'existence et le mode de vie en Suisse. Comme jusqu'ici, on distingue entre le fait d'être intégré et le fait d'être familiarisé, le premier terme se rapportant à la capacité d'insertion dans la vie sociale en Suisse, le deuxième faisant référence plus spécialement aux compétences linguistiques et à la capacité du candidat à participer aux processus de décision politique en tant que futur citoyen suisse.

Il faut encore comprendre par intégration (let. a) l'accueil de la personne étrangère dans la société suisse et sa disposition à s'insérer dans le contexte social, sans pour autant abandonner son identité et sa nationalité. Aujourd'hui, l'intégration est généralement considérée comme un processus de rapprochement réciproque entre la population indigène et la population étrangère, qui présuppose tant la disposition des étrangers à s'intégrer que la volonté des Suisses d'être ouverts à cette intégration (voir art. 3 de l'ordonnance sur l'intégration des étrangers, RS 142.205). Selon la pratique de naturalisation actuelle, il n'est nullement exigé du candidat qu'il renonce à son identité pour se «glisser dans la peau d'un autre». Pour cette raison, l'entretien

22. Voir ATF 138 I 242, du 12 juin 2012. La jurisprudence a ainsi atténué l'impact de ce critère en reconnaissant, dans un cas d'espèce, comme susceptible d'être naturalisé un parent qui, s'occupant de son enfant handicapé, n'a pas le temps de nouer et d'approfondir des contacts avec le tissu local, mais qui est toutefois bien intégré dans son contexte professionnel. À ce sujet, voir aussi les arrêts du 12 juin 2012 publiés aux ATF 138 I 242, consid. 5.3, et 138 I 305, consid. 4.4.

23. FF 2002 1815-1899, spéc. 1844. 
de relations avec le pays d'origine - p. ex. avec la famille restée au pays ou en raison de la propriété de biens fonciers dans ce pays - ne s'oppose pas à une naturalisation. Pour cette raison aussi, le terme d'intégration est préférable à celui d'assimilation qui est aujourd'hui considéré comme obsolète.

Ce passage indique que l'intégration consiste donc dans le fait de s'insérer dans la société suisse, dans la vie sociale et professionnelle, alors que le présupposé de la familiarisation, qui comprend les connaissances linguistiques, ainsi que celles relatives aux droits politiques, se réfere plutôt à la participation des futures citoyennes et citoyens aux processus politiques et décisionnels du pays. On peut donc constater une évolution dans les critères requis pour se faire naturaliser. L'identité culturelle ainsi que la provenance de la personne requérante ne sont plus mises en question.

Si l'on s'en tient à ce message, l'intégration consiste aussi dans le fait d'accueillir la personne étrangère dans la communauté suisse et dans sa disponibilité à s'insérer dans le contexte social du pays sans toutefois renoncer à ses propres caractéristiques et à sa propre nationalité ${ }^{24}$.

Pour ce qui est des connaissances linguistiques, le Manuel précise que le candidat doit être en mesure de communiquer, de manière suffisante, dans sa vie quotidienne, dans une des langues nationales, et d'être ainsi autonome ${ }^{25}$. À propos de la faculté de "communiquer dans l'une des langues nationales", le Conseil fédéral précise que les cantons peuvent fixer des exigences plus étendues que celles prévues par la Confédération ${ }^{26}$.

Alors que les communes sont en principe libres de déterminer la procédure d'évaluation des connaissances linguistiques et de définir le niveau requis, il est toutefois exigé d'elles qu'elles établissent de manière explicite leurs attentes en la matière, par le biais d'une information préalable destinée à garantir l'égalité de traitement et le

24. Dans un arrêt du 28 septembre 2015 (C-7590/2014), le Tribunal administratif fédéral a contribué à spécifier les exigences posées par les lettres a à d de l'art. $14 \mathrm{LN}$, en se référant au Manuel du SEM. En particulier, il a défini l'exigence de l'intégration dans la communauté suisse (let. a) comme la disponibilité de l'étranger à s'intégrer dans le contexte social du pays, sans renoncer à sa propre identité. L'intégration comprend une série de critères, comme le respect de la Constitution, de l'ordre juridique suisse, la participation à la vie sociale, les échanges avec la population locale, la vie professionnelle, les connaissances linguistiques (voir p. 15).

25. Voir le Manuel du SEM, op. cit., p. 25.

26. FF 2011 2639-2698, spéc. 2648. 
droit d'être entendu (art. 8 et 29 al. 2 de la Constitution fédérale) ${ }^{27}$. Bien que les connaissances linguistiques constituent un élément clé de l'intégration, il est toutefois possible de compenser un éventuel manque par d'autres éléments, comme l'a souligné la jurisprudence du Tribunal administratif fédéral dans le cas d'une personne qui cultive des contacts avec la population suisse dans une langue autre que nationale ${ }^{28}$.

Un autre aspect important est celui du recours aux prestations sociales. Le Manuel du SEM précise à ce propos que le recours à l'assistance ou à l'aide sociale, aux prestations de l'assurance invalidité ou de l'assurance chômage, lorsque les autres critères de l'intégration sont réunis, ne signifie pas automatiquement le rejet d'une requête de naturalisation dans la procédure devant les autorités fédérales. Cela ne se produit, effectivement, que quand le recours à ces aides est imputable à la personne candidate ou qu'il existe des indices de fraude ${ }^{29}$. De son côté, le Conseil fédéral fonde le principe de l'autosuffisance financière - l'aptitude à subvenir à ses propres besoins - sur le critère de la «[v]olonté de participer à la vie économique et d'acquérir une formation ${ }^{30}$.

Le Tribunal fédéral a toutefois précisé aussi que le principe de l'indépendance financière ne s'applique pas de la même manière pour les personnes qui souffrent de déficiences physiques ou psychiques, ou encore d'une maladie chronique ou d'un handicap qui les empêchent de subvenir à leurs propres besoins ${ }^{31}$.

27. Voir à ce propos l'ATF 137 I 235, du 13 avril 2011, ainsi que l'ATF 140 I 99, du 14 février 2014. Dans le cadre d'une demande de naturalisation d'une famille de quatre personnes rejetée par les autorités communales de Weiningen $(\mathrm{ZH})$, le Tribunal fédéral a rappelé que les intéressés doivent être informés sur les procédures et les conditions matérielles de la naturalisation, en estimant, dans le cas d'espèce, qu'il y a eu violation de ces règles de procédure (droit d'être entendu, droit à un procès équitable et principe de la bonne foi). Affirmant le principe qu'une autorité communale viole le principe de la bonne foi et l'équité de la procédure (art. 5 al. 3 et 29 Cst.) lorsqu'elle invite le candidat à un entretien destiné à faire connaissance et à exposer les motifs de sa demande alors qu'elle procède en réalité à un examen inopiné de ses compétences générales, le Tribunal fédéral a donc admis le recours, annulé la décision attaquée et renvoyé la cause à l'autorité inférieure pour nouvelle décision.

28. Voir l'arrêt du Tribunal administratif fédéral C-5286/2007. Toujours en ce qui concerne le critère linguistique, ce tribunal a clarifié qu'il n'y a pas discrimination pour motif de religion lorsqu'une autorité refuse la naturalisation en raison des connaissances linguistiques insuffisantes. Voir aussi l'ATF 132 I 167, du 10 mai 2006, consid. 4.

29. Voir le Manuel du SEM, op. cit., p. 25.

30. FF 2011 2639-2698, spéc. 2648-2649.

31. Voir ATF 135 I 49, du 16 décembre 2008, consid. 6.1. Le Tribunal fédéral a toutefois aussi souligné, dans un autre cas, que le refus de la naturalisation ne saurait être considéré en l'occurrence comme une discrimination liée à l'origine modeste de la famille; voir ATF 136 I 309, du 25 août 2010, consid. 4.4. 
Le critère du respect de l'ordre juridique suisse, selon le Manuel du SEM (par. 4.7.3.), se réfere à la situation pénale financière ${ }^{32}$. De son côté, le Conseil fédéral requiert, comme manifestations du respect de l'ordre juridique suisse, une conduite irréprochable du point de vue du droit pénal et des obligations financières ${ }^{33}$. Une inscription au casier judiciaire représente donc un obstacle à la naturalisation à moins qu'il ne s'agisse d'infractions mineures qui, comme telles, n'ont pas d'incidence sur la procédure ${ }^{34}$.

Pour ce qui est des questions financières, même si le Manuel du SEM consacre plusieurs pages à cette question ${ }^{35}$, on admet généralement, comme le précise le Conseil fédéral, que le «respect des obligations financières (faillites en cours, dettes fiscales) doit en principe être laissé à l'appréciation des autorités communales et cantonales", du moins en matière de naturalisation ordinaire ${ }^{36}$. Dans sa jurisprudence, le Tribunal fédéral a considéré qu'il n'était pas arbitraire d'admettre que l'observation des obligations de droit public - en particulier l'absence de retard de dettes en matière d'impôts - était une condition indispensable à l'octroi de la naturalisation, dans la mesure où telle était la volonté clairement exprimée par le législateur, tant fédéral que cantonal, telle qu'elle ressortait des travaux préparatoires des dispositions en cause ${ }^{37}$.

Indépendamment du type de naturalisation (ordinaire ou facilitée), la loi exige que la personne requérante ne compromette pas la sûreté intérieure ou extérieure de la Suisse ${ }^{38}$. En effet, comme le souligne le

32. Voir le Manuel du SEM, op. cit., p. 34.

33. FF 2002 1815-1899, spéc. 1845. Dans ce sens, voir l'arrêt du Tribunal administratif fédéral C-1148/2013, du 6 février 2014, à propos d'un candidat ukrainien qui, durant son séjour en Suisse, a subi de nombreuses condamnations pénales en matière de circulation routière. De telles infractions représentent, selon le juge fédéral, un obstacle à l'autorisation fédérale de naturalisation en tant que violation de l'art. $14 \mathrm{LN}$.

34. À ce propos, voir en détail le Manuel du SEM, pp. 34 et ss. En principe, en cas de condamnation, plus longue est la peine, plus elle représente un obstacle à la naturalisation. Selon l'arrêt du Tribunal administratif fédéral C-2642/2011, par exemple, l'ouverture d'une procédure pénale pour conduite en état d'ébriété représente un obstacle à l'obtention de l'autorisation fédérale de naturalisation.

35. Voir le Manuel du SEM, op. cit., pp. 40-44.

36. FF 2002 1815-1899, spéc. 1845; cf. aussi Manuel du SEM, op. cit., notamment p. 40.

37. Voir par exemple les arrêts 1D_1/2017, du 24 mai 2017 (A. gegen Bürgerrechtskommission Hochdorf), 1D_6/2016, du 5 janvier 2017 (A. contre Municipalité de Crissier), 1D_3/2012, du 29 avril 2013 (A.X. gegen Bürgerrat der Gemeinde Riehen), 1D_4/2008, du 5 septembre 2008 (A.X. gegen Gemeinde Wolhusen) ; voir aussi l'arrêt 1D_4/2011, du 27 septembre 2011 (A. contre Conseil d'État de la République et canton de Neuchâtel), où le Tribunal fédéral admet néanmoins le recours et renvoie l'affaire aux autorités cantonales, considérant que les faits et les données relatifs aux dettes fiscales de l'intéressée étaient établis de manière par trop lacunaire.

38. Cette exigence est formulée aussi bien à l'art. 14 let. d qu'à l'art. 18 al. 1 let d LN, ainsi qu’à l'art. 26 al. 1 let. c, pour le cas de la réintégration. 
message concernant la modification de la loi sur la nationalité du 26 août 1987, il serait contraire aux intérêts du pays de conférer la naturalisation à une personne susceptible d'être expulsée ${ }^{39}$. Ce critère est étendu aux membres de la famille du candidat ${ }^{40}$.

Des indices concrets sont suffisants pour admettre la mise en danger, il n'est pas nécessaire que la menace se soit déjà produite ${ }^{41}$. À l'inverse, si le danger est passé, il n'y a pas de raison de ne pas accorder la naturalisation ${ }^{42}$.

\subsubsection{LA PROTECTION JURIDICTIONNELLE}

\section{EN CAS DE REFUS DE LA NATURALISATION}

En dépit de la spécification progressive des critères prévus pour l'acquisition de la nationalité, les marges d'appréciation des autorités (fédérale et cantonales) n'en sont pas pour autant diminuées, dans la mesure où celles-ci peuvent toujours refuser la naturalisation ${ }^{43}$.

39. FF 1987 III 285-336, spéc. 297. Sow et Mahon, 2014, ad art. 14 LN, nos 32 à 39, pp. 56-57, interprètent ce critère de la mise en danger de la sûreté intérieure ou extérieure de la Suisse comme la mise en danger de la souveraineté étatique dans le domaine militaire et politique. À ce sujet, voir l'arrêt du Tribunal administratif fédéral C-1124/2006, du 21 août 2009, de même que l'arrêt C-1123-2006, du 12 septembre 2008, consid. 6.2.3.

40. Voir l'arrêt du Tribunal administratif fédéral C-1121/2006, du 21 août 2009, consid. 5.6., selon lequel la Confédération peut vérifier l'aptitude du candidat s'il existe des éléments extérieurs qui ne regardent pas seulement la personne du requérant, mais d'autres membres de sa famille, pour autant que ces éléments soient aptes à compromettre la sûreté intérieure ou extérieure de la Suisse.

41. Sow et Mahon, 2014, ad art. 14 LN, nos 32 à 39, pp. 56-57.

42. Sur la question, voir l'arrêt C-2946/2008 du Tribunal administratif fédéral, du 21 juin 2011. Dans le cas d'une jeune étudiante originaire des États-Unis, qui avait entretenu des relations avec l'IRA, association qui figure depuis toujours sur la liste de celles qui représentent une menace pour le pays, la Cour a estimé qu'ayant pris ses distances avec l'organisation en question, la candidate ne représente pas une menace pour la Suisse (cf. consid. 6.1). Un autre arrêt récent du Tribunal administratif, C-3195/2012, du 2 septembre 2015, en affirmant le principe de "vraisemblance» et de la libre appréciation des preuves en matière d'examen des faits juridiquement pertinents de la part de l'autorité administrative, a accueilli partiellement le recours d'un ressortissant italien auquel la naturalisation facilitée avait été refusée au motif que ses antécédents pénaux représentaient une menace pour la sécurité intérieure et extérieure de la Suisse. Le Tribunal administratif fédéral, en jugeant ouverte la question de la violation par le requérant de l'art. 26 al. 1 let. c LN (respect de l'ordre juridique suisse), a condamné le mode de décision du SEM, lequel, en déléguant à la Fedpol le soin de mener les investigations, aurait basé sa décision sur des suppositions et non sur des investigations circonstanciées et individualisées sur le soupçon d'appartenance à la 'Ndrangheta calabraise (consid. 7.6). Voir aussi l'arrêt C-3769/2011, du 6 octobre 2014, dans lequel le Tribunal administratif fait à l'ODM (SEM) le même reproche de n'avoir pas évalué de manière complète la situation du candidat pour établir si celui-ci respectait ou non les conditions prévues à l'art. 14 let. $\mathrm{d}$ LN, et d'avoir fondé sa propre décision de refus de la naturalisation uniquement sur des informations fournies par le SRC (Service de renseignement de la Confédération), violant ainsi le droit du requérant à une procédure équitable (recours admis, le requérant remplissant les autres exigences prévues par la loi).

43. Le Tribunal administratif fédéral a rappelé que bien que les candidats remplissent toutes les exigences légales, il n'existe pas un véritable droit à la naturalisation. Voir à ce propos, par exemple, l'arrêt C-2642/2011, du 19 septembre 2012, consid. 5.3., et l'arrêt C-1124/2006, du 21 août 2009, consid. 4.3. Sur la question, cf. Sow et Mahon, 2014, ad art. 14 LN, nos 6 et 7, p. 48 . 
Naturellement, il est prévu que les appréciations des autorités s’inscrivent dans le respect des garanties constitutionnelles et des autres dispositions légales ${ }^{44}$. Comme il n'existe pas de garanties spécifiques en faveur des personnes candidates à la naturalisation, les protections qu'il est possible d'invoquer sont celles de l'égalité de traitement et de l'interdiction des discriminations (art. 8 Cst.), ainsi que de la prohibition de l'arbitraire (art. 9 Cst) et, sur le plan des garanties de procédure, le droit d'être entendu et, en lien avec celui-ci, le droit d'obtenir une décision motivée (art. 29 Cst.) ${ }^{45}$. Parmi ces garanties, le droit d'être entendu joue un rôle clé, avec son corollaire, le droit de consulter le dossier (art. 26 à 28 PA), et celui de s'exprimer au sujet de celui-ci (art. 29 à 33 PA). L'article 15b LN avait déjà consacré, depuis une dizaine d'années, l'obligation de motiver un éventuel refus de la naturalisation ${ }^{46}$, indépendamment de l'organe dont émane une telle décision, concrétisant ainsi l'article 29 al. 2 de la Constitution:
Art. 15b - Obligation de motiver la décision
${ }^{1}$ Tout rejet d'une demande de naturalisation doit être motivé.
${ }^{2}$ Une demande de naturalisation ne peut être rejetée par les élec- teurs que si elle a fait l'objet d'une proposition de rejet motivée.

L'obligation de motiver le refus de naturalisation vaut donc aussi pour les décisions prises en votation populaire ${ }^{47}$.

L'autre disposition extrêmement importante en matière de naturalisation et de droit des étrangers est l'interdiction du déni de justice, qui implique pour les autorités l'obligation de prendre

44. L'autorité abuse de sa liberté d'appréciation lorsqu'elle n'utilise pas correctement son pouvoir, débordant de sa marge d'appréciation; voir à ce propos Sow et Mahon, ad art. 14 $L N$, nos 7 et 8 , pp. 48-49.

45. Dans son arrêt 1D_3/2012, du 29 avril 2013, le Tribunal fédéral a retenu qu'il n'y avait en l'occurrence pas violation de l'art. 9, ni de l'art. 8 de la Constitution du moment que le non-respect de l'ordre juridique suisse (en raison d'une amende non payée) constitue un indice d'intégration manquée.

46. Larticle 15b LN a été introduit par la loi fédérale du 21 décembre 2007 (Procédure dans les cantons/Recours devant un tribunal cantonal), en vigueur depuis le $1^{\text {er }}$ janvier 2009 (RO 2008 5911). Ainsi que l'explique Gutzwiller, cette disposition représente une concrétisation au niveau de la procédure de naturalisation de l'art. 29 al. 2 de la Constitution fédérale (droit d'être entendu); cf. Gutzwiller, 2014, ad art. 15b, nos 1 et 3, pp. 78-79. Sur la possibilité que le candidat fasse recours à partir de la connaissance des éléments de la décision, voir ATF $129 \mathrm{I}$ 232, du 9 juillet 2003. Voir en outre Gutzwiller, 2008, pp. 373 ss.

47. Dans un arrêt de 2012, le Tribunal fédéral a reconnu, en relation avec l'obligation de motiver les décisions de naturalisation, que les candidats disposent d'une position juridique suffisamment précise qui leur permet d'invoquer devant lui la protection contre l'arbitraire et le droit à l'égalité de traitement. Voir l'ATF 138 I 305, du 12 juin 2012, précité, consid. 1.4.6. Voir aussi l'ATF 132 I 196, du 10 mai 2006. Sur le droit d'être entendu, voir ATF 124 II 132, du 20 mars 1998. 
ses décisions avec une certaine célérité, sans tarder, ou au moins de concéder certaines autorisations ou prorogations à titre transitoire, dans l'attente de la décision, lorsque l'intérêt de la personne concernée le requiert ${ }^{48}$. Enfin, il faut rappeler que toutes les autres dispositions générales de la loi fédérale sur la procédure administrative (PA) peuvent également être invoquées par le recourant en cas de refus de la naturalisation ${ }^{49}$. Naturellement, en plus d'avoir des droits et des garanties de procédure, la personne requérante a aussi des devoirs, notamment celui de collaborer avec les autorités administratives, en communiquant les faits en sa faveur, mais aussi, dans l'idéal, en sa défaveur, que l'autorité devra apprécier. Sur le plan procédural, le Titre 5 de la nouvelle loi sur la nationalité prévoit, à l'article 47, que l'autorité compétente pour juger des recours contre les décisions de l'administration fédérale et des autorités cantonales de dernière instance est celle désignée par les dispositions générales de la procédure fédérale ${ }^{50}$. Pour le reste, aux termes de l'article $46 \mathrm{LN}$, il appartient aux cantons de désigner les autorités compétentes pour connaître, en tant qu'autorité cantonale de dernière instance, des recours dirigés contre les décisions de refus de la naturalisation ordinaire. Du moment qu'existent les voies de recours ainsi mentionnées (dans les cas de décisions prises arbitrairement ou en violation de l'interdiction de discrimination), la doctrine récente se demande s'il ne convient pas d'admettre l'existence d'un quasi-droit à la naturalisation, et ce contrairement à l'affirmation de principe traditionnelle selon laquelle il n'y a pas de droit à la naturalisation qui puisse être invoqué comme tel par les candidates et les candidats ${ }^{51}$.

48. Voir Jeanneret et Mahon, 2017, ad art. 112 LEtr, no 7, pp. 1288-1289.

49. L'abréviation PA se réfere à la loi fédérale du 20 décembre 1968 sur la procédure administrative (RS 172.021). Les garanties applicables sont prévues par la loi du 17 juin 2005 sur le Tribunal administratif fédéral (LTAF, RS 173.32) et la loi du 17 juin 2005 sur le Tribunal fédéral (LTF, RS 173.110), en plus des garanties constitutionnelles.

50. Voir en outre l'art. 46 de la même loi, qui prévoit qu'il doit y avoir, dans les cantons, une possibilité de recours devant une autorité cantonale de dernière instance.

51. Sur la notion de "quasi-droit", voir Sow et Mahon, 2014, ad art. $14 L N$, no 8, p. 49. Voir aussi l'arrêt C-2642/2011 du Tribunal administratif fédéral, du 19 septembre 2012, consid. 5.3. Comme on le sait, le renforcement de la protection juridique en cas de naturalisation a été développé à partir de l'arrêt Emmen du Tribunal fédéral, du 9 juillet 2003 (ATF 129 I 217), lequel, en plus de constituer un précédent juridictionnel, a provoqué une forte réaction politique, avec une modification consécutive du cadre légal. Le principe du "quasi-droit" à la naturalisation a été développé en lien avec la naturalisation facilitée, dans la mesure où la loi prévoit la possibilité de recourir au Tribunal administratif fédéral, contre un éventuel refus de la naturalisation facilitée par le SEM, pour faire vérifier le respect des conditions des art. 27 et 28 de la loi sur la nationalité (LN de 1990); voir par exemple, en matière de naturalisation facilitée, les ATF 130 II 169, du 22 janvier 2004, 130 II 482, du 7 septembre 2004, 132 II 113, du 22 novembre 2005, 135 II 1, du 12 novembre 2008, et 135 II 161, du 29 janvier 2009. 


\subsubsection{SYNTHÈSE INTERMÉDIAIRE}

L'analyse des dispositions législatives concernant la procédure de naturalisation a permis de mettre en évidence l'œuvre du législateur suisse dans la spécification progressive et toujours accrue des critères d'acquisition de la naturalisation. Le critère de l'aptitude, formulé dans les années 1950, a ainsi fait place au - ou a été accompagné du - critère de l'intégration, dans les années 1990, lequel a fait place, dès 2014, à celui d'intégration réussie. Ces dispositions se fondent sur les précisions données précédemment par le Conseil fédéral quant à la compréhension des notions de familiarisation et d'accoutumance comme éléments permettant de juger de l'aptitude à la naturalisation et de la notion d'intégration, précisant que la familiarisation dépend, précisément, de l'intégration. Il avait ajouté que l'expression «être familiarisé» devait être préférée à celle d' "être assimilé", dans la mesure où la première n'implique pas de connotation négative. Elle n'exige pas de la personne requérante qu'elle "change de peau», mais elle suppose la réalisation d'une sorte de mariage entre deux cultures, la culture suisse et la culture d'origine de la personne candidate ${ }^{52}$. Lors de l'étape la plus récente du processus de réforme, il a par ailleurs été spécifié que l'intégration doit être réussie, puisque la naturalisation représente l'étape ultime du processus d'intégration, et l'on a clarifié ainsi le rapport de continuité, mais aussi la différence existant entre l'intégration telle que décrite dans la loi sur la nationalité et l'intégration dans la loi sur les étrangers ${ }^{53}$. En outre, sur le plan linguistique, le critère quelque peu vétuste de s'être "accoutumé» au mode de vie et aux usages suisses (prévu par l'ancienne loi sur la nationalité) a été remplacé par celui de s'être "familiarisé» avec les conditions de vie en Suisse (art. 11 let. b nLN).

Passant du point de vue politico-législatif à celui de la pratique jurisprudentielle, nous avons pu observer comment les critères d'ordre individuel, culturel et social, d'une part, et les critères d'ordre public, d'autre part, constituent les bases de l'évaluation

52. Voir le Message concernant la modification de la loi sur la nationalité, du 26 août 1987, FF 1987 III 285: le fait de s'être familiarisé est une conséquence de l'intégration et consiste en l'adoption des modes de vie suisses. Un tel concept est renforcé dans le Message concernant la révision totale de la loi fédérale sur la nationalité suisse, du 5 avril 2011, FF 20112639 , où il est précisé que l'on peut parler de familiarisation à partir du moment où existent des contacts réguliers avec les voisins suisses ou une participation à la vie associative avec un ancrage local. Cette familiarisation s'exprime également à travers les connaissances de la géographie, de l'histoire et des institutions locales.

53. Voir FF 20112639. 
de l'intégration des candidates et candidats à la naturalisation. Parmi les critères du premier type, le facteur linguistique, parfois combiné avec le facteur de la vie associative et celui des connaissances culturelles, semble jouer un rôle clé dans la procédure de naturalisation. Les convictions religieuses de la personne candidate ne représentent en revanche pas, en règle générale, un signe de défaut d'intégration. Pour ce qui est des critères du second type, l'absence de mise en danger de la sûreté interne et externe, ainsi que l'autonomie financière et la participation à la vie économique (l'absence de dépendance de l'aide sociale) représentent les principaux facteurs d'une intégration considérée comme réussie. Enfin, sur le plan des garanties de procédure, l'obligation de motivation de la décision de refus de la naturalisation prévue par l'article 15b LN, liée à d'autres formes de garanties telles que la prohibition des décisions arbitraires et l'interdiction du déni de justice - qui ouvrent la voie du recours auprès des autorités cantonales et fédérales - conduisent une partie de la doctrine à admettre l'existence d'un quasi-droit à la naturalisation. Un tel quasi-droit est en outre confirmé par la nouvelle loi sur la nationalité, de 2014, qui impose la désignation d'autorités de recours au niveau cantonal déjà contre les refus de naturalisation ordinaire (art. 46 et $47 \mathrm{nLN}$ ).

\subsection{LA NOTION D'INTÉGRATION DANS LA LOI FÉDÉRALE SUR LES ÉTRANGERS (LETR)}

Le second ensemble législatif au sein duquel est formulé, au niveau fédéral, le critère de l'intégration est celui de la loi sur les étrangers. À partir des années 1980, l'intégration des étrangers en Suisse est conçue comme un devoir ou une tâche de l'État: elle doit aller au-delà de la seule sphère du social et de l'assistance, comme cela avait été le cas jusqu’à ce moment, pour acquérir une dimension politique nationale, fondée sur l'encouragement à l'intégration du point de vue linguistique et culturel et sur la familiarisation avec les conditions de vie en Suisse. L'intégration est d'autre part considérée comme un devoir pour toute personne aspirant à séjourner sur le territoire suisse. C'est ainsi qu'avec l'adoption de la nouvelle loi sur les étrangers du 16 décembre 2005, l'intention du Conseil fédéral était d'ancrer dans la loi des principes importants destinés à promouvoir l'intégration des étrangers et étrangères là où la 
législation précédente (la LSEE) se limitait à prévoir une simple réglementation-cadre ${ }^{54}$. À la lecture du message du Conseil fédéral concernant cette loi, il est évident que l'intégration est conçue, en principe, comme un concept de type évolutif, qui ne peut être défini a priori ${ }^{55}$.

Nous examinerons ici, dans un premier temps, les divers critères de l'intégration tels qu'ils sont posés par la loi et interprétés par la pratique administrative fédérale (3.1), avant d'analyser le rôle de la jurisprudence, notamment du Tribunal fédéral, dans l'interprétation et la délimitation de cette notion (3.2). Un bref excursus sera consacré à la question de la protection judiciaire en la matière (3.3), avant que ne soient proposées quelques conclusions intermédiaires (3.4).

\subsubsection{LES CONDITIONS DE L'INTÉGRATION}

À la lecture de l'article 4 LEtr, il apparaît que l'intégration est envisagée comme un processus dual, basé sur l'accueil de la communauté suisse, d'une part, et sur la disponibilité à s'intégrer, d'autre part. L'objectif principal d'un tel processus réside dans la réalisation d'une coexistence empreinte de respect et de tolérance réciproques, comme on peut le lire littéralement dans la disposition ${ }^{56}$ :

54. Voir le message concernant la loi sur les étrangers, du 8 mars 2002, FF 2002 3469-3644. Il s'agit toutefois, dans celle contenue dans la loi sur les étrangers, d'une réglementation non exhaustive du moment que le statut des étrangers se trouve régi par des normes du droit international public, du droit européen - du fait des accords conclus par la Suisse et l'Union européenne et ses États membres -, du droit fédéral et du droit cantonal. Voir à ce propos Nguyen, 2017, ad art. 1 LEtr. Pour ce qui est de la loi sur le séjour et l'établissement des étrangers, dont il a déjà été question dans le chapitre 1, voir aussi le Message du Conseil fédéral à l'Assemblée fédérale concernant un projet de loi sur le séjour et l'établissement des étrangers, du 17 juin 1929, FF 1929 I 929-949.

55. Dans les instructions du SEM à propos de l'intégration (version de janvier 2015), il était précisé que «le Conseil fédéral et le Parlement ont volontairement évité de définir légalement l'intégration "étant donné que l'acception sociale et les représentations de l'intégration peuvent évoluer avec le temps"»; cette précision n’a toutefois pas été reprise dans la version des mêmes directives mise à jour en janvier 2019 (cf. https://www.sem.admin.ch/dam/data/ sem/rechtsgrundlagen/weisungen/integration/weisungen-integration-f.pdf); janvier 2015); il faut préciser toutefois que cette version 2019 renvoie, pour ce qui est des critères d'intégration dans le cadre de la nouvelle LEI, à une autre directive, elle aussi actualisée en 2019, la Directive Domaine des étrangers (ou Directive LEI), laquelle reprend, à son chiffre 3.3 (pp. 41 à 51), toutes les instructions du SEM en matière d'intégration et de critères d'intégration, pour le secteur du droit des étrangers (sur cette directive, cf. https://www.sem.admin.ch/sem/fr/home/ publiservice/weisungen-kreisschreiben/auslaenderbereich.html, consulté le 29 janvier 2020). On précisera que le texte de ce chapitre se fonde, en principe, et sauf indication contraire, sur la version 2015 des directives, en vigueur au moment de notre recherche.

56. Pour un commentaire de l'art. 4, voir Achermann et Amarelle, 2017, ad art. 4 LEtr, pp. 24-44. 


\begin{abstract}
Art. 4 Intégration
${ }^{1}$ L'intégration des étrangers vise à favoriser la coexistence des populations suisse et étrangère sur la base des valeurs constitutionnelles ainsi que le respect et la tolérance mutuels.

${ }^{2}$ Elle doit permettre aux étrangers dont le séjour est légal et durable de participer à la vie économique, sociale et culturelle.

${ }^{3}$ L'intégration suppose d'une part que les étrangers sont disposés à s'intégrer, d'autre part que la population suisse fait preuve d'ouverture à leur égard.

${ }^{4} \mathrm{Il}$ est indispensable que les étrangers se familiarisent avec la société et le mode de vie en Suisse et, en particulier, qu'ils apprennent une langue nationale.
\end{abstract}

Afin de concrétiser cette disposition, l'ordonnance sur l'intégration des étrangers (OIE) du 24 octobre 2007 précise, à son article 4, la contribution attendue des étrangères et des étrangers. Celle-ci se manifeste à travers: "a) le respect de l'ordre juridique et des valeurs de la Constitution fédérale; b) l'apprentissage de la langue nationale parlée sur le lieu de domicile; c) la connaissance du mode de vie suisse; d) la volonté de participer à la vie économique et d'acquérir une formation. ${ }^{57}$ Selon une partie de la doctrine, de telles dispositions renverraient à une sorte de "contrat» migratoire entre la société d'accueil et la personne étrangère, laquelle assume l'obligation de respecter les lois écrites et les règles sociales ${ }^{58}$. D'autre part, bien qu'il n'existe pas une obligation du même genre à charge de la population suisse, autre qu'une idée générique d'ouverture de sa part, il existe dans l'ordre juridique suisse diverses normes, comme l'interdiction des discriminations (art. 8 al. 2 Cst.), qui, ainsi qu'on le verra encore, peuvent avoir pour objet de protéger les étrangères et les étrangers. En outre, en vue de la réussite du processus d'intégration, les autorités fédérales et cantonales, ainsi que communales, sont tenues d'assumer un devoir d'information. Selon le Conseil fédéral, une "intégration réussie présuppose une bonne information au sujet des conditions de vie et de travail, des droits et obligations qui prévalent en Suisse " ${ }^{59}$. D'où la formulation

57. RS 142.205.

58. Dans le message concernant la loi fédérale sur les étrangers, du 8 mars 2002 (FF 2002 3469-3644, 3555), il est fait mention d'une "obligation de s'intégrer" proposée en phase de consultation, obligation dont la légitimité a toutefois été mise en question en lien avec le risque de lésion de la liberté personnelle qu'elle pouvait impliquer.

59. Message concernant la loi sur les étrangers, du 8 mars 2002, FF $20023469,3558$. 
de l'art. 56 LEtr qui prévoit qu'une série d'informations soit fournie par la Confédération, les cantons et les communes, sur les conditions de vie et de travail en Suisse et les droits et obligations (al. 1). Les étrangères et les étrangers sont également informés sur le pays d'accueil et sur la politique migratoire ${ }^{60}$.

Les principes en matière d'intégration restent génériques, comme le confirment quelques-unes des dispositions prévues par la loi : que l'on considère, par exemple, l'exigence d'évaluer «la situation personnelle de l'étranger, ainsi que [...] son degré d'intégration» (art. 96) ou encore l' «intégration réussie» (art. 50 al. 1 let. a), le «degré» d'intégration (art. 30 al. 1 let. b et art. 54). Le caractère vague de telles expressions contribue à rendre indéfini le concept d'intégration, accordant ainsi, comme on le sait, une marge d'appréciation importante aux autorités cantonales et communales appelées à juger du degré d'intégration des personnes qui demandent un permis ou un changement de statut ${ }^{61}$. Comme dans le cadre de la loi sur la nationalité, la pratique administrative fédérale ainsi que la jurisprudence récente en matière d'intégration jouent à cet égard un rôle clé de clarification. Ainsi, selon les instructions du SEM, d'une manière générale, l'intégration ${ }^{62}$ :

peut être qualifiée de réussie lorsque les valeurs statistiques enregistrées dans les domaines sociaux, tels que la formation, l'exercice d'une activité lucrative ou la délinquance, sont comparables à celles relatives aux Suisses d'âge, de sexe, de situation sociale et économique, de statut familial et de formation professionnelle comparables. Un manque de connaissances et de compétences (p. ex., faibles connaissances linguistiques, formation professionnelle insuffisante, peu de contacts, manque de motivation, etc.)

60. Les détails concernant les informations à fournir aux étrangers figurent dans l'ordonnance sur l'intégration (OIE, RS 142.205). Voir à ce propos Achermann, Amarelle et Kurt, 2017, ad art. 56, nos 9 à 15, pp. 528-531. Le principe d'une information «appropriée» constitue un des points cardinaux de la mise en œuvre des «PIC», instruments actuels de la politique cantonale d'intégration; voir les indications du SEM sur l'«Encouragement de l'intégration», https:// www.bfm.admin.ch/content/bfm/fr/home/themen/integration/foerderung.html.

61. Sur le thème du pouvoir discrétionnaire (pouvoir d'appréciation) des autorités, voir Bigler et Bussy, 2017, ad art. 96 LEtr, nos 8 à 12, pp. 1038-1040, selon lesquels " [e]xerce son pouvoir d'appréciation dans le choix entre plusieurs conséquences juridiques possibles l'autorité qui conditionne l'octroi d'une autorisation de séjour à la participation à un cours de langue ou à un cours d'intégration (art. 54 LEtr) ou qui adresse un avertissement (art. 96 al. 2 LEtr). Dans tous les cas, l'état de fait à la base du pouvoir d'appréciation lui sert de cadre juridique» (no 11, p. 1040).

62. Voir les instructions du SEM sur l'intégration, op. cit., dans la version de 2015, pp. 2-3 (la version de 2019 n'a pas repris l'ensemble de ce passage). 
peut être à l'origine de différences quant aux chances de succès. Ces différences peuvent aussi être dues à des obstacles d'ordre juridique ou à certaines formes de discrimination qui empêchent les étrangers d'accéder au même titre que les autres citoyens à la vie économique, sociale et culturelle. De telles circonstances sont susceptibles d'être prises en considération lors de l'évaluation du niveau de réussite de l'intégration.

Pour déterminer la réussite et le degré de l'intégration dans des cas individuels, le SEM se fonde par exemple sur les principes fixés par l'ordonnance sur l'intégration des étrangers (OIE) et a élaboré une série d'instructions en la matière. En particulier, sur la base de l'article 4 OIE, il a développé et concrétisé les critères suivants comme paramètres de l'intégration: le respect des valeurs de la Constitution fédérale, le respect de la sécurité et de l'ordre public ainsi que de la sécurité intérieure et extérieure de la Suisse, la volonté de participer à la vie économique et d'acquérir une formation, la connaissance des conditions de vie en Suisse, l'apprentissage de la langue nationale parlée au lieu de résidence, le fait de s'être accoutumé au mode de vie et aux usages suisses.

Premièrement, sont considérés comme indicateurs du respect des valeurs de la Constitution au sens dudit article 4 les éléments suivants ${ }^{63}$ :

- le fait que l'expression concrète du comportement de l'étranger ne traduise pas une attitude de fond qui soit en contradiction avec les valeurs fondamentales de la Constitution fédérale;

- l'absence de déclarations publiques ou de comportements enregistrés par les autorités qui soient en violation avec les valeurs fondamentales de la Constitution fédérale que sont le monopole exercé par l'État sur la puissance publique, l'égalité entre femmes et hommes, la liberté personnelle (p. ex. des enfants), ainsi que l'intégrité physique et psychique d'autrui (membres de la famille compris).

Le deuxième critère prévu à l'article 4 let. a OIE est celui de la sécurité et de l'ordre publics, qui réside dans le respect de l'ordre juridique suisse, des biens juridiques et des personnes (vie, santé, liberté, propriété, etc.) ainsi que des institutions de l'État. L'ordre

63. Comme le précisent les directives du SEM (version 2015), sont pris en considération uniquement "les faits avérés consignés dans le dossier de l'intéressé», op. cit., p. 3. 
public, à son tour, se subdivise en un ordre juridique objectif, dont les indicateurs sont représentés par une réputation irréprochable - selon l'extrait du casier judiciaire -, et les représentations de l'ordre public (dimension subjective). Ce second élément comprend, selon les directives du SEM, «l'ensemble des représentations non écrites de l'ordre, dont le respect doit être considéré, selon l'opinion sociale et éthique dominante, comme une condition inéluctable d'une cohabitation humaine ordonnée»; en font partie notamment ${ }^{64}$ :

- le respect des décisions des autorités et l'observation de ses obligations de droit public ou de ses engagements privés (absence de poursuites ou de dette fiscale, paiement ponctuel des pensions alimentaires, etc.);

- la coopération avec les autorités (aide sociale, autorités fiscales, etc.) $[\ldots]$.

Le troisième critère, celui de la participation à la vie économique, prévu par l'article 4 let. d OIE, s'appuie sur la vérification de la participation effective par l'acquisition et l'exercice d'une profession. À titre exceptionnel, il est possible de se contenter de la seule manifestation de l'intention ou de la volonté de réussir, démontrée dans un passé récent ou au présent. Il faut aussi considérer les éventuels empêchements de travailler ou d'acquérir une profession qui ne sont pas imputables à une faute individuelle, par exemple lorsqu'un tel empêchement dérive d'une interdiction de travailler, d'un problème grave de santé ou de la violence physique ou psychique subie ${ }^{65}$.

Pour le SEM, le recours à l'aide sociale n'est pas un critère d'intégration manquée ou insuffisante, mais peut constituer un motif légal de révocation ou de non-renouvellement du permis de séjour. Il faut donc tenir compte des circonstances particulières et fournir les preuves d'une formation en cours (contrat d'apprentissage,

64. Ibid., p. 4. La demande de l'intéressé ne doit pas contenir de référence à des incidents d'aucune sorte (par exemple, blâme de la part des autorités scolaires, non-respect de convocations, etc.).

65. Ibid., p. 5. Selon les instructions du SEM, les éléments qui indiquent la volonté de participer à la vie économique sont les suivants: "Un contrat de travail non résilié (photocopie du contrat de travail, accompagnée d'une attestation de travail récente) ou la preuve de l'indépendance économique de l'intéressé (p. ex., activité lucrative indépendante); la preuve des efforts fournis pour trouver un emploi (annonce à l'ORP); des postes de travail temporaires (postes intérimaires, emplois temporaires) ou la confirmation de gains intermédiaires démontrant la volonté de subvenir par soi-même à ses propres besoins. " 
attestation de l'institut de formation) ou de la participation à des cours ou des mesures de perfectionnement.

Le quatrième critère, prévu à l'article 4 let. C OIE, celui de la connaissance des conditions de vie en Suisse, est interprété en termes atténués, ou de manière moins sévère, en comparaison de son appréciation dans le contexte de la loi sur la nationalité ${ }^{66}$. Il est attendu de la personne étrangère qu'elle connaisse les conditions de vie en Suisse, mais l'absence de preuve du respect de cette condition ne peut être interprétée à son détriment dans la mesure où, en pratique, cette connaissance est difficilement démontrable.

Le cinquième critère, linguistique, revêt une importance presqu'égale dans les deux lois, la loi sur la nationalité et la loi sur les étrangers, comme cela ressort de l'article 4 let. b OIE ${ }^{67}$.

En règle générale, la personne étrangère doit apprendre la langue nationale parlée dans son lieu de résidence. Des dérogations à ce principe peuvent toutefois être admises, notamment en cas de changement de domicile à destination d'une autre région linguistique du pays. Les cantons dans lesquels sont parlées deux langues officielles peuvent décider laquelle des deux langues doit être apprise ou en laisser le choix. En principe, sont exigées ${ }^{68}$ :

- une preuve de participation régulière et active à des cours de langues (attestation du chargé de cours) ou

- des tests de langue réussis effectués auprès d'un établissement de formation reconnu $[\ldots]$;

- une attestation de formation en cas de fréquentation d'une école en Suisse.

Le Manuel du SEM mentionne un sixième critère en plus de ceux mentionnés, qui se rattache aux articles 14 et 26 de la loi sur la nationalité, et qui veut que l'étrangère ou l'étranger se soit accoutumé "au mode de vie et aux usages suisses», en précisant toutefois explicitement que ce critère ne concerne que la procédure de naturalisation, et non le droit ordinaire des étrangers. Les indicateurs d'une telle accoutumance sont ${ }^{69}$ :

66. Ibid., p. 5 .

67. Selon le SEM, il faut considérer la situation particulière de chacun (analphabétisme, niveau de formation, charge professionnelle, devoir d'assistance familiale, etc.). Ibid., pp. 5-6.

68. Ibid., p. 6.

69. Ibid. 
- la connaissance des faits marquants de l'histoire ou des particularités géographiques et politiques de la Suisse;

- la connaissance de faits marquants sur le plan local, cantonal et national;

- les contacts établis avec ses voisins suisses;

- l'affiliation à une société ancrée localement ou l'engagement en faveur d'une association de ce type; une certaine indépendance manifestée dans la manière de mener sa vie, par exemple une mobilité autonome ou le fait de posséder son propre compte bancaire.

Comment nous le préciserons dans les conclusions, la mention de ce sixième critère parmi les autres critères d'intégration risque de donner lieu à une confusion de la part des destinataires de ces directives.

\subsubsection{L'INTÉGRATION NE COÏNCIDE PAS AVEC L'ASSIMILATION:}

\section{LE RÔLE DE LA JURISPRUDENCE}

Les arrêts du Tribunal fédéral et du Tribunal administratif fédéral en matière d'intégration concernent aussi bien les critères d'ordre individuel, culturel et social ${ }^{70}$ (le critère linguistique, avant tout, et la participation à la vie sociale, ainsi que les convictions religieuses) que les critères d'ordre public ${ }^{71}$ (comme la violation de l'ordre juridique et de la sûreté interne ou externe de la Suisse). À la différence de la jurisprudence en matière de naturalisation, ce sont ici les critères du second ordre qui font l'objet de la majorité des décisions relatives à l'intégration ${ }^{72}$. La jurisprudence concernant l'application de la loi sur les étrangers met en effet moins l'accent sur les questions relatives aux connaissances des usages et de la culture suisses que ne le fait la jurisprudence concernant la naturalisation. Le processus d'intégration n'est pas envisagé comme un processus d'assimilation, qui nécessite une adaptation aux usages et aux coutumes du pays, comme c'est

70. Voir l'ATF 135 I 79, du 24 octobre 2008, consid. 7.2., qui concerne la question de la dispense des cours de natation pour deux jeunes garçons de religion musulmane, dans lequel le Tribunal fédéral met en balance le respect de la liberté religieuse, d'une part, et le principe de l'intégration (dont l'apprentissage de la natation représente une manifestation), d'autre part.

71. Voir l'arrêt C-881/2012, du 18 septembre 2014, par lequel le Tribunal administratif fédéral, tout en reconnaissant l'intégration professionnelle de l'intéressé, considère que celui-ci ne remplit pas le critère du respect de l'ordre juridique, pour avoir subi plusieurs condamnations pénales. De même, les solutions retenues dans les arrêts 2C_87/2014, du Tribunal fédéral, du 27 octobre 2014, et C-2515/2012, du Tribunal administratif fédéral, du 6 octobre 2014.

72. Voir l'arrêt du Tribunal fédéral 2C_915/2010, du 4 mai 2011: le Service des migrations du canton de Neuchâtel avait refusé la demande d'autorisation de séjour du requérant en raison de condamnations pour vol. Dans ce cas, le Tribunal fédéral a estimé que l'intérêt public à protéger la Suisse l'emporte sur l'intérêt privé du recourant (malade du sida) et de son conjoint à pouvoir vivre sur le territoire suisse. 
le cas pour les personnes candidates à la naturalisation ${ }^{73}$. Dans une optique d'égalité des chances entre personnes étrangères et personnes suisses, le facteur linguistique semble en revanche revêtir la même importance dans la loi sur les étrangers et dans la loi sur la nationalité. Dans la première, ce facteur figure parmi les critères énumérés à l'article 53, en matière d'encouragement à l'intégration. Un tel critère revêt, selon Achermann et Amarelle, une importance particulière du moment que, avec les critères relatifs au respect de l'ordre juridique suisse (soit, en substance, l'absence de condamnation pénale) et de la participation à la vie économique (en substance, l'absence de recours à une allocation de chômage de longue durée), il est objectivement vérifiable. Le financement de la participation aux cours de langue et les autres mesures d'intégration sont prévus par l'article 53 LEtr, disposition qui, comme on l'a vu, régit la contribution de l'État (aux niveaux fédéral, cantonal et communal) à l'encouragement et à la promotion de l'intégration ${ }^{74}$. Un tel encouragement est concrétisé, en particulier, à travers les structures ordinaires telles que l'école, le monde du travail, le système de sécurité sociale et de santé. Le degré d'intégration peut, enfin, jouer un rôle s'agissant de l'octroi d'un permis d'établissement (au sens de l'article 54 LEtr), jusqu'à requérir, dans certains cas, l'intégration réussie ${ }^{75}$. Le retrait du permis (art. 63 LEtr) et l'expulsion (art. 68 LEtr) ne sont en revanche possibles qu'aux conditions prévues par la loi ${ }^{76}$.

73. Voir l'ATF 134 II 1, du 25 février 2008, concernant l'expulsion d'un citoyen turc qui habitait en Suisse depuis vingt-cinq ans environ et était suspecté d'avoir contraint sa fille à un mariage forcé. Le Tribunal fédéral a jugé la mesure non conforme au principe de proportionnalité. Dans l'arrêt 2C_37/2014, du 15 août 2014, toutefois, le Tribunal fédéral n'a pas reconnu l'intégration du requérant, provenant du Kosovo, du moment que celui-ci, malgré les années passées en Suisse, n’avait démontré aucune connaissance de base de l'organisation de la vie en Suisse, ni n'avait de contacts avec la population locale; en outre, ses connaissances du français étaient très approximatives, au point que les autorités cantonales qui avaient examiné sa demande avaient dû recourir à un interprète. Dans le même sens, voir aussi l'arrêt du Tribunal administratif fédéral C-528/2012, du 28 juillet 2014.

74. Selon l'art. 53 LEtr, les autorités doivent tenir compte des objectifs d'intégration en créant des conditions qui y soient propices et en encourageant l'intégration dans certains domaines particuliers, ainsi qu'en tenant compte, par exemple, des exigences spécifiques des femmes, des enfants et des adolescents, ou encore des partenariats sociaux, des organisations non gouvernementales et des organisations d'étrangers. Voir Achermann et Amarelle, 2017, ad art. 53, pp. 488-496.

75. Selon l'art. 54 LEtr, même l'octroi d'une autorisation d'établissement ou d'une autorisation de séjour de courte durée peut être subordonnée à la participation à un cours de langue ou d'intégration. Une telle obligation peut être fixée dans une convention d'intégration (au sens de l'art. 54 al. 1). Voir Achermann et Kurt, 2017, ad art. 54, pp. 487-513.

76. En matière de révocation des décisions, voir l'ATF 137 II 297, du 15 avril 2011, selon lequel, au sens de l'art. 62 let. b et de l'art. 63 al. 1 let. b LStr, le cumul de nombreuses peines plus courtes, en lien avec une peine privative de liberté de longue durée, représente un motif de révocation en tant que menace gravissime à la sécurité et à l'ordre public. 


\subsubsection{LA PROTECTION JURIDICTIONNELLE}

\section{DES PERSONNES ÉTRANGÈRES}

Toutes les garanties de procédure administrative et judiciaire prévues dans le cadre de l'État de droit pour garantir les droits humains fondamentaux sont en principe applicables aux personnes étrangères, comme elles le sont, on l'a vu, pour les personnes qui sont candidates à la naturalisation (ch. 2.4): du droit d'être entendu à l'obligation de motivation des décisions. De même, sont applicables les dispositions générales de la loi fédérale sur la procédure administrative $(\mathrm{PA})^{77}$, de la loi sur le Tribunal administratif fédéral (LTAF) et de la loi sur le Tribunal fédéral (LTF) ${ }^{78}$. Des dispositions spécifiques de procédure sont par ailleurs aussi prévues par la loi sur les étrangers elle-même, et elles peuvent déroger aux règles générales de procédure évoquées plus haut. Selon l'article $112 \mathrm{LEtr}$, ce sont les règles de procédure cantonales qui s'appliquent lorsque la mission de mettre en œuvre la loi sur les étrangers incombe aux cantons, cela bien entendu dans le respect des règles fédérales et internationales.

L'autorité de recours de première instance contre les décisions de l'administration fédérale est en principe le Tribunal administratif fédéral (selon l'art. 47 al. 1 let. b PA). Dans la mesure où la décision concerne la sûreté interne ou externe de la Suisse, la protection diplomatique ou d'autres cas concernant les relations extérieures, l'autorité de recours est en revanche l'autorité administrative supérieure (art. 47 al. 1 let. d PA; art. 32 al. 1 let. a LTAF). L'interdiction d'entrée et l'expulsion, prononcées par l'Office fédéral de la police (en application de l'art. 67 al. 4 ou de l'art. 68 LEtr), doivent en conséquence faire exceptionnellement l'objet d'un recours auprès du Département fédéral de justice et police, à moins qu'il ne s'agisse d'une ou d'un ressortissant européen, qui peut se prévaloir des accords bilatéraux, spécialement de l'Accord sur la libre circulation des personnes (ALCP). Dans ce cas, le recours doit être adressé au Tribunal administratif fédéral, du moment que le droit international confere un droit à ce que la cause soit jugée par un tribunal. Enfin, dans le cas où c'est le Conseil fédéral qui opterait pour une expulsion, le recours est adressé directement au Tribunal fédéral.

77. Loi fédérale sur la procédure administrative, du 20 décembre 1968 (RS 172.021).

78. Il s'agit respectivement de la loi sur le Tribunal administratif fédéral, du 17 juin 2005 (RS 173.32), et de la loi sur le Tribunal fédéral, du 17 juin 2005 (RS 173.110). 
Les décisions susmentionnées prises par le Département fédéral de justice et police sont sujettes à recours uniquement auprès du Conseil fédéral (art. 72 let. a PA). Au contraire, les jugements sur recours du Tribunal administratif fédéral en matière de droit des étrangers peuvent normalement faire l'objet d'un recours en matière de droit public au Tribunal fédéral, sous réserve des cas dans lesquels ce recours n'est pas ouvert, comme en cas de refus d'entrée en Suisse ou de refus de visa, ou encore en cas de refus d'une autorisation de séjour à laquelle le droit fédéral ou le droit international ne confere pas un droit (voir art. 83 let. c LTF).

Pour ce qui est de la procédure devant les autorités cantonales, elle est, comme on l'a dit, régie par les lois cantonales de procédure administrative et d'application de la loi sur les étrangers, dans le respect des règles du droit fédéral et du droit international, des droits fondamentaux, ainsi que de la loi sur les étrangers ellemême, laquelle détermine des règles de procédure applicables aux cantons (voir par exemple les art. 64 al. 2, 74 al. 3, 78 al. 4 et 80 al. 2 LEtr). Les décisions des services cantonaux compétents peuvent généralement faire l'objet d'un recours à l'autorité de surveillance supérieure, soit auprès du département au sein duquel est intégré le service en question ou au Conseil d'État. En général, c'est cette autorité qui est dotée des pleins pouvoirs dans l'examen du fond (en ce qui concerne les faits et le droit), ainsi que de l'opportunité de la décision. Enfin, un recours auprès du Tribunal administratif cantonal est possible, celui-ci n'ayant cependant pas toujours la compétence d'examiner l'opportunité ${ }^{79}$.

Les jugements des autorités judiciaires cantonales de dernière instance peuvent en principe faire l'objet d'un recours en matière de droit public au Tribunal fédéral, sauf dans les nombreuses hypothèses dans lesquelles, en matière de droit des étrangers, ce recours n'est - en vertu de la loi sur le Tribunal fédéral - pas ouvert, comme dans les cas de refus d'une autorisation de séjour à laquelle le droit fédéral ou le droit international ne confere pas un droit (voir art. 83 let. c, ch. 2, LTF). Dans de telles hypothèses, le seul remède au plan fédéral concerne alors l'éventuelle violation de droits constitutionnels - à l'exclusion des violations de la loi sur les étrangers -, qui peut être porté au Tribunal fédéral par la voie (extraordinaire) du recours constitutionnel subsidiaire (art. 116

79. Cf. Jeannerat et Mahon, 2017, ad art. 112 LEtr, no 21, p. 1296. 
LTF). Le recourant devra pouvoir se prévaloir, pour emprunter cette voie, d'un intérêt juridiquement protégé (art. 115 LTF) et il ne pourra invoquer que la violation des garanties de procédure prévues par la Constitution fédérale ou la violation, par la décision contestée, de l'interdiction des discriminations (art. 8 al. 2 Cst.) ${ }^{80}$.

\subsubsection{SYNTHÈSE INTERMÉDIAIRE}

Nous avons vu comment, en instaurant une réglementation spécifique du droit des étrangers, la loi sur les étrangers, le Conseil fédéral a introduit des principes clés en matière d'intégration, renonçant dans le même temps à définir de manière précise cette notion. Le caractère vague des dispositions législatives a ainsi été quelque peu précisé par la pratique des autorités administratives fédérales et la jurisprudence, qui ont contribué à donner signification à cette notion d'intégration. Les instructions du Secrétariat d'État aux migrations semblaient prévoir à cet égard deux types de critères: des critères qu'il qualifiait d'objectifs, qui ont trait au respect des valeurs de la Constitution, ainsi que le respect de la sûreté interne et externe du pays et de l'ordre public; des critères qu'il qualifiait de subjectifs, qui concernent la volonté de participer à la vie économique, la connaissance des conditions de vie suisses et l'apprentissage de la langue nationale parlée sur le lieu de résidence. La prévision d'un sixième critère dans les instructions du SEM - même avec la précision que ce critère est applicable exclusivement au cas de naturalisation -, qui porte sur la familiarisation avec les modes de vie suisses, était propre à éveiller quelque perplexité. Ce sixième critère nous ramène en effet à l'interrogation de départ sur la légitimité d'une attente d'intégration qui soit identique, en termes de degré d'intégration, pour une personne candidate à la naturalisation et pour une (simple) demande de permis de séjour. Par contre, la distinction opérée par la jurisprudence, qui, dans le cas de la loi sur les étrangers, met moins l'accent sur le critère de la connaissance des us et coutumes suisses que dans le cadre de la loi sur la nationalité, semble plus nette, laissant ainsi place à une application implicite du principe de proportionnalité s'agissant de l'appréciation du degré d'intégration en lien avec un permis de séjour ou une demande de naturalisation. Pour ce qui

80. Jeannerat et Mahon (2017, ad art. 112 LEtr, no 23, p. 1297) critiquent l'absence de protection juridique, au niveau fédéral, qui peut en résulter, en particulier contre des décisions arbitraires de la part des autorités cantonales. 
est des critères qui relèvent de l'ordre public, un poids supérieur est accordé, dans le cadre de la LEtr, au critère du respect de l'ordre juridique suisse et de la sûreté intérieure et extérieure du pays. En revanche, le critère linguistique semble ou semblait revêtir une importance semblable dans les deux lois, cela surtout en fonction de la réalisation d'une égalité des chances entre personnes étrangères et suisses et de l'effectivité du processus d'intégration. Ainsi, même l'obtention d'une autorisation d'établissement ou d'une autorisation de séjour de courte durée peut être subordonnée à la participation à un cours de langue ou d'intégration, obligation qui dans certains cantons peut être prévue par une convention d'intégration.

Il faut préciser toutefois, à ce propos, que l'harmonisation de la notion d'intégration opérée par les révisions récentes, déjà évoquées, dans les deux domaines législatifs du droit de la naturalisation et du droit des étrangers devrait conduire à une meilleure coordination et à une plus grande cohérence des exigences en la matière, selon un modèle que l'on peut qualifier de "graduel», postulant que les exigences en matière d'intégration sont d'autant plus élevées que sont importants les droits conférés à la personne étrangère en vertu de son statut juridique, comme l'expriment les nouvelles directives du Secrétariat d'État aux migrations ${ }^{81}$. Ainsi, par exemple, ces directives indiquent désormais clairement, et de manière schématique, les exigences croissantes ("graduelles") en matière de connaissances linguistiques qui sont requises en fonction du type de statut, allant de l'admission provisoire à la naturalisation en passant par l'autorisation de séjour et celle d'établissement ${ }^{82}$.

\subsection{LA LOI SUR L'INTÉGRATION ET LA COHÉSION CULTURELLE DU CANTON DE NEUCHÂTEL}

En vertu de leur responsabilité solidaire en matière d'intégration sociale, culturelle et politique des étrangères et étrangers, et dans le cadre de leurs compétences respectives (art. 121 Cst), la Confédération, les cantons et les communes œuvrent de concert pour l'intégration, en conformité avec l'article 53 LEtr, intitulé «Encouragement de l'intégration».

81. Voir la nouvelle Directive Domaine des étrangers (Directives LEI), déjà citée dans la note 56, version 2019, ch. 3.3.1 (https://www.sem.admin.ch/sem/fr/home/publiservice/ weisungen-kreisschreiben/auslaenderbereich.html, consulté le 29 janvier 2020).

82. Ibid., ch. 3.3.1.3, p. 43. 
Les pratiques cantonales dans le domaine de l'intégration varient, en fonction notamment des diverses réglementations dont les cantons se dotent et des politiques régionales et locales. Une étude récente du Forum suisse pour l'étude des migrations menée sur les différentes politiques d'intégration cantonales a montré une oscillation entre des tendances inclusives et exclusives de la part des cantons ${ }^{83}$. Elle parle de tendances inclusives là où les cantons fixent des exigences relativement peu élevées pour les immigrés et de tendances exclusives lorsque, au contraire, ces exigences d'intégration sont élevées. L'étude rend compte des pratiques obligatoires en matière d'intégration, comme celles liées à l'utilisation des conventions d'intégration, instrument introduit par l'ordonnance sur l'intégration des étrangers (OIE) du 24 octobre 2007, pour certaines catégories de personnes étrangères, en particulier celles qui ne disposent pas d'un droit de séjour stable en Suisse. De telles conventions, qui visent principalement à l'apprentissage de la langue ou à la participation à un cours d'intégration, se fondent sur un contrat entre l'autorité et la personne étrangère, qui accepte ainsi une série d'obligations. À la différence de la philosophie plus impérative ou volontariste qui est à la base des conventions d'intégration appliquées dans certains cantons, des pratiques plus libérales comme celles qui existent dans le canton de Neuchâtel, fondées sur des programmes d'éducation civique et sur la participation active des associations d'étrangers, démontrent que l'intégration peut aussi être entendue comme une forme de coexistence entre différents groupes de population, plutôt que comme un processus unilatéral d'adaptation de l'un (celui des personnes étrangères) aux autres (la population suisse).

Nous examinerons ici, dans un premier temps, le principe d'égale dignité que le canton de Neuchâtel cherche à mettre en avant et en pratique dans sa politique d'intégration des étrangères et des étrangers (ch. 4.1), avant d'analyser l'un des instruments de cette politique d'intégration (ch. 4.2) et le rôle du Service de la cohésion multiculturelle en la matière (ch. 4.3).

83. Il s'agit de l'enquête conduite par le Forum suisse pour l'étude des migrations et de la population (SFM) sur demande de la Commission fédérale pour les questions de migration (CFM) : Les marges de manouvre au sein du fédéralisme: La politique de migration dans les cantons, Berne 2011, https://www.ekm.admin.ch/dam/data/ekm/dokumentation/ materialien/mat_foederalismus_f.pdf (consulté le 16 janvier 2020). On renvoie en outre au dossier du SFM Migrazione e Integrazione: focus sulla Svizzera romanda (n. 8, 2013), pour une analyse de la politique migratoire suisse, dont le canton de Neuchâtel fait partie. Voir en particulier la contribution de Wichmann, pp. 63 ss. 


\subsubsection{LE PRINCIPE D'ÉGALE DIGNITÉ}

Le canton de Neuchâtel a été le premier à se doter d'une Loi sur l'intégration et la cohésion multiculturelle, entrée en vigueur le 26 août 1996 et modifiée en $2013^{84}$. Cette loi a pour but de favoriser la cohésion sociale ainsi que l'égale dignité et le bienêtre de toute personne habitant le canton, sur la base de relations harmonieuses et de la compréhension réciproque entre population migrante qui s'installe à Neuchâtel et population résidente. À la lecture de l'article premier, en particulier, il apparaît que l'intégration est conçue comme un processus d'adaptation réciproque entre populations suisse et étrangère, à travers la participation à la vie économique, sociale, culturelle et politique:

${ }^{1}$ La présente loi a pour but de favoriser la cohésion sociale, l'égale dignité et le bien-être de toute personne vivant dans le canton de Neuchâtel, notamment par des relations harmonieuses et la compréhension mutuelle entre les populations suisse et étrangères ou issues de la migration.

${ }^{2}$ Elle encourage la recherche et l'application de solutions pour l'intégration interculturelle, la pleine participation des personnes issues de la migration à la société et, de façon plus générale, tend à promouvoir l'égalité des droits et devoirs ainsi que la non-discrimination pour tout un chacun dans les limites de la Constitution et de la loi.

Selon l'un des auteurs de cette législation, Thomas Facchinetti, alors délégué aux étrangers du canton, l'objectif du dispositif mis en place par la loi correspond ainsi, plutôt, à un processus d'ajustement réciproque entre personnes étrangères et personnes nationales ${ }^{85}$ :

Dans l'optique neuchâteloise, l'intégration des étrangers désigne un processus d'adaptation mutuelle, aux niveaux individuels et collectifs, des populations suisses et étrangères. Elle implique la participation à la vie économique, sociale, culturelle et politique. Il ne s'agit pas d'un alignement unilatéral des étrangers à une sorte de «moule helvétique» qui nierait les racines et références

85. Voir Facchinetti, 2011, et aussi la page du COSM : http://www.ne.ch/autorites/ DFS/SCSP/prevention/IPM/cosm.pdf. 
identitaires multiples des populations en présence, mais d'un ajustement réciproque et permanent des uns et des autres. Dans ce domaine très sensible de la vie en commun de populations provenant d'horizons passablement diversifiés, les équilibres et la cohésion sociale ne sont jamais définitifs et il faut veiller en permanence à les renouveler.

Dans un tel cadre politique et législatif, les autorités neuchâteloises ambitionnent de promouvoir le développement de relations de cohabitation harmonieuses, en encourageant la recherche de solutions favorables à l'intégration, cela à partir des marges de manœuvre et de l'autonomie dont les cantons et les communes disposent vis-à-vis du droit fédéral ${ }^{86}$.

\subsubsection{LES INSTRUMENTS D'UNE POLITIQUE LIBÉRALE:}

\section{LA CHARTE DE LA CITOYENNETÉ}

Par la promulgation de la loi sur l'intégration en 1996, les autorités ont confirmé la tradition ou la réputation libérale qui caractérise le canton de Neuchâtel. Cette réputation repose sur le fait que les personnes étrangères résidant à Neuchâtel ont obtenu le droit de vote au plan communal en 1848 . Elles se sont vu reconnaître le droit de vote en matière cantonale en 2000 et, en 2007, le droit d'éligibilité en matière communale, tant pour le législatif que pour l'exécutif communal. Cette empreinte libérale de la politique neuchâteloise en la matière est, du reste, inscrite à l'article premier de la nouvelle Constitution du canton, du 24 septembre 2000, qui définit le canton comme une "république démocratique, laïque, sociale et garante des droits fondamentaux ${ }^{87}$. Cela est explicité par la Charte de la citoyenneté 88 , document qui a été adopté en mars 2008 et est accessible en diverses versions linguistiques. Il a pour objectif d'aider les personnes nouvellement arrivées, ainsi que celles qui résident dans le canton, à mieux comprendre les principes et les fondements de la Constitution neuchâteloise. À Neuchâtel,

86. Voir aussi ibid., pp. 3-4.

87. RS 131.233.

88. La Charte de la citoyenneté, qui est fournie par le Département de l'économie - Service de la cohésion multiculturelle du canton de Neuchâtel, a été élaborée en 2007. Elle a été créée par la volonté du Conseil d'État dans le but d'expliciter les fondements et les principes essentiels de l'organisation démocratique aux nouveaux arrivants dans le canton. Elle est consultable en ligne: https://www.ne.ch/autorites/DEAS/ COSM/conseil-information/PublishingImages/Pages/Charte-de-la-citoyennet $\%$ C $3 \% \mathrm{~A} 9 /$ mementoA4FRA.pdf (consulté le 29 janvier 2020). 
l'État libéral et démocratique se caractérise par la concession de droits fondamentaux et de libertés à ses habitantes et habitants, ainsi que par la participation démocratique à la formation de la volonté et à l'exercice du pouvoir ${ }^{89}$ :

Les fondements et principes d'un État sont généralement transcrits dans les lois, qui sont des règles juridiques concrètes, que chacun, Suisse comme étranger, est tenu de respecter. Même s'il n'y a aucune obligation juridique d'adhérer aux principes eux-mêmes, il faut quand même, pour qu'un État comme Neuchâtel puisse fonctionner, qu'une majorité de la population les connaisse, les respecte et s'engage à les défendre.

Pour définir l'État de Neuchâtel comme un État libéral, la Charte met l'accent sur la dignité de la personne: l'État reconnaît à la personne une sphère d'indépendance et de liberté qui se traduit par la protection de ses droits fondamentaux, au cœur desquels figure ce qui constitue le fondement et le premier de tous les droits fondamentaux inhérents à la personne, la dignité humaine ${ }^{90}$ :

La dignité humaine, c'est le droit de ne pas être traité de manière inhumaine et dégradante, le droit d'être traité comme un être humain et non comme une chose. Elle représente le noyau intangible de la liberté personnelle et protège par exemple contre la torture et tout autre traitement cruel ou inhumain.

Autour de la dignité humaine, tous les autres «droits fondamentaux" peuvent être divisés en différentes catégories: libertés (libertés de la sphère personnelle, libertés de communication et libertés économiques), garanties de l'État de droit et droits sociaux.

Ainsi, la notion de dignité humaine, entrée dans la Constitution fédérale de 1999, est considérée comme la norme fondamentale en matière de protection des droits fondamentaux. Selon l'article 7 de la Constitution fédérale, «[l]a dignité humaine est respectée et protégée " ${ }^{91}$. Au plan cantonal, on retrouve une disposition

89. Ce passage est tiré de la page 1 de l'opuscule Fondements et principes de la république et canton de Neuchâtel, partie intégrante de la Charte: https://www.ne.ch/autorites/DEAS/ COSM/conseil-information/PublishingImages/Pages/Charte-de-la-citoyennet\%C3\%A9/ mementoA4FRA.pdf (consulté le 29 janvier 2020).

90. Ibid., p. 3.

91. Ibid. 
analogue dans la Constitution neuchâteloise, qui, à son article 7 , stipule: «La dignité humaine est respectée et protégée.» La correspondance, tant dans les formulations de ces deux dispositions, fédérale et cantonale, que dans leur place, ne relève pas d'une simple coïncidence; elle est voulue du moment que les cantons ne peuvent prévoir des garanties qui vont moins loin que celles accordées par le droit fédéral. En outre, la reconnaissance de droits fondamentaux au niveau cantonal s'inscrit dans une longue tradition qui coïncide avec la tradition démocratique suisse et l'attachement aux libertés individuelles ${ }^{92}$. En effet, dans les années 1960 déjà, le constitutionnaliste neuchâtelois Jean-François Aubert retenait que l'égalité entre personnes étrangères et Suisses était inhérente à la nature des choses, à partir du principe de la dignité humaine, sans que soient nécessaires des traités entre les peuples pour la garantir ${ }^{93}$.

En tant qu'étape finale du processus d'intégration, la Charte de la citoyenneté du canton de Neuchâtel prévoit l'acquisition des droits de citoyenneté par le biais de la naturalisation comme le moyen d'exercer pleinement le droit de vote (aussi au plan national) et de participer à la vie politique ${ }^{94}$. S'établir dans le canton de Neuchâtel signifie s'impliquer concrètement dans la vie de la société en participant à la vie politique ou manifester sa propre appartenance à la collectivité d'accueil en acquérant la nationalité suisse.

\subsubsection{LA MISE EN CEUVRE DE LA LOI: LE COSM}

La mise en œuvre de la loi sur l'intégration et la cohésion multiculturelle du 26 août 1996 est confiée au Service cantonal de la cohésion multiculturelle (COSM), dont la mission est de favoriser des relations harmonieuses entre personnes suisses et étrangères, l'idée de cohésion exprimant le fait que ce service de l'État est un service ouvert à tout le monde, et que ce qui y est fait pour les étrangers est fait pour toute la population ${ }^{95}$.

Pour réaliser cette mission, le Service travaille en collaboration avec d'autres services cantonaux et avec l'implication active des

92. Cf. la newsletter du CSDH, Jubilé, du 24 novembre 2014, «La démocratie suisse et son attachement aux libertés individuelles", http://www.skmr.ch/frz/domaines/questionsinstitutionnelles/nouvelles/suisse-attachement-libertes-individuelles.html?zur=93 (consulté le 16 janvier 2020).

93. Voir Aubert, 1958, spéc. p. 250.

94. Voir la page 6 de la Charte de la citoyenneté, op. cit.

95. Les fonctions du COSM sont consultables à partir du rapport d'activité: http:// www.ne.ch/autorites/DEAS/COSM/Documents/CICM_COSM-rapportactivites2016V. pdf (consulté le 16 janvier 2020). 
communautés et associations de personnes étrangères, dans le cadre de la Communauté pour l'intégration et la cohésion multiculturelle (CICM), organisme qui existe depuis plusieurs années et qui constitue un espace de dialogue qui réunit toutes les parties prenantes pour permettre la discussion des problématiques spécifiques et la recherche de solutions ${ }^{96}$.

C'est la même loi cantonale de 1996 qui prévoit une base légale spécifique à la mise en ouvre des actions liées non seulement au Service de la cohésion multiculturelle, mais aussi à la Communauté pour l'intégration et la cohésion multiculturelle ${ }^{97}$. Le mandat de cette dernière est d'étudier les phénomènes liés à la migration internationale et de favoriser l'intégration des populations étrangères dans la société neuchâteloise. Elle se compose de sous-commissions, par exemple une sous-commission en matière de migrations féminines ou un groupe de contact avec la communauté musulmane, qui s'occupe de questions liées à la discrimination ${ }^{98}$.

\subsection{SYNTHÈSE: LE DÉFI DE L'INTÉGRATION RÉUSSIE}

Après l'analyse historico-juridique, proposée dans le chapitre 3, des principales étapes politico-législatives de l'évolution de la réglementation de la présence et du statut des étrangers sur le territoire suisse, qui a permis de mettre en lumière les diverses significations que la notion d'intégration a assumées au cours du temps dans les débats politiques et dans les réglementations normatives successives, le présent chapitre s'est attaché, dans une perspective plus actuelle, à mettre en évidence les significations que la notion d'intégration a acquises dans la pratique administrative et dans la jurisprudence.

Comme on l'a déjà dit, étant donné la similitude des critères d'intégration posés par la loi sur la nationalité, d'une part, et par la loi sur les étrangers, de l'autre, ces deux législations ont récemment fait l'objet de débats et de projets de révision, qui ont notamment eu pour but ou pour effet d'harmoniser la notion d'intégration (3.3). C'est ainsi que la nouvelle loi issue de la révision totale de la loi sur la nationalité, adoptée en 2014 et entrée en vigueur en 2018, pose le principe d'une intégration réussie comme condition d'obtention de la naturalisation.

96. Ibid.

97. Pour ce qui est des fonctions de la CICM, voir le rapport d'activité du COSM, op. cit., pp. 6 et ss.

98. Ibid., p. 7. 
Une telle exigence d'une intégration « réussie» pour les personnes candidates à la nationalité suisse pourrait faire penser que l'intégration est conçue comme un processus graduel, qui tient compte d'exigences différentes selon le statut de la personne concernée, c'est-à-dire selon que celle-ci revendique l'obtention d'un permis de séjour du droit des étrangers ou, au contraire, qu'elle aspire à la naturalisation au sens de la loi sur la nationalité. On peut éventuellement trouver la confirmation d'une telle conception dans la jurisprudence, selon laquelle intégration ne coïncide pas avec assimilation (3.2), en ce sens qu'il y a lieu de procéder à une interprétation différenciée du concept d'intégration dans ces deux législations. Une telle différenciation ne ressort toutefois pas toujours des attentes ou des interprétations qui se trouvent dans la pratique des autorités fédérales. Le fait de retenir, comme le faisaient les directives du Secrétariat d'État aux migrations, le critère de la familiarisation avec les "modes de vie suisses" aussi bien dans la législation sur les personnes étrangères que dans la loi sur la nationalité semble indiquer qu'il subsiste ou subsistait une certaine confusion, ou du moins une superposition, entre les critères d'application de la loi sur la nationalité et de la loi sur les étrangers. Comme on l'a vu, toutefois, l'harmonisation de la notion d'intégration opérée par les révisions récentes dans les deux domaines législatifs du droit de la naturalisation et du droit des étrangers devrait conduire à une meilleure coordination et à une plus grande cohérence des exigences en la matière, sur la base d'une gradation des exigences. En outre, l'analyse conjointe de la législation fédérale et du droit cantonal - avec l'exemple du canton de Neuchâtel - a permis de montrer comment l'autonomie politique et législative dont disposent les cantons peut être mise au service d'une conception plus ou moins libérale de l'intégration, fondée sur une forme de coexistence civique et sur un principe d'égale dignité plutôt que sur des attentes et des exigences d'adaptation unilatérale des uns envers les autres, comme c'est le cas dans d'autres cantons.

Enfin, dans les termes de ce que nous avons défini comme une institution de réinvention de la personne étrangère (dans le chapitre 1, puis dans le chapitre 3), nous avons examiné en détail les critères destinés à homologuer, en quelque sorte, la conduite de la personne étrangère candidate à la naturalisation, en la comparant avec celle des personnes de nationalité suisse. 
Nous avons ainsi vu comment, avec l'adoption de l'ancienne loi sur la nationalité, ce mécanisme de transformation s'est concrétisé, à partir des années 1950, à travers l'introduction du critère de l'aptitude à devenir suisse (art. $14 \mathrm{LN}$ ), critère qui requérait l'assimilation de la personne candidate - de son caractère, de ses habitudes, de son style de vie, de sa personnalité - aux us et coutumes suisses. En d'autres termes, cette personne candidate à la naturalisation devait devenir une personne de confiance aux yeux de ses futurs concitoyennes et concitoyens.

Par la suite, à partir des années 1990, l'exigence de transformation de l'identité de la personne candidate (sa culture, sa personnalité) a paru s'atténuer, avec l'introduction d'un autre critère, celui de l'intégration. Ici, l'accent semble se déplacer de l'évaluation de la personnalité de la candidate ou du candidat à la naturalisation à ses attitudes dans la vie quotidienne: capacité de communiquer dans une langue nationale, capacité d'autonomie sur le plan professionnel et capacité d'insertion sociale.

Dans l'actualité la plus récente, avec la réforme de 2014, en vigueur dès 2018, l'exigence de transformation de la personne réapparaît. S’il n'est certes plus possible de revenir au critère de l'assimilation, il n'est pas moins exigé que l'intégration soit réussie, critère qui est examiné avant la naturalisation. Celle-ci finit ainsi par devenir l'étape ultime du parcours d'intégration de la personne étrangère dans le pays d'accueil. 


\title{
5. DÉMÊLER L'ÉCHEVEAU: LA PROCÉDURE EN SCHÉMA
}

\author{
ANNE LAVANCHY ET PASCAL MAHON
}

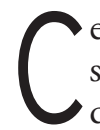

e chapitre présente, de manière systématique, synthétique et synoptique, la procédure qui permet à une personne candidate d'obtenir, par sa naturalisation, la nationalité suisse. Il résulte d'un besoin de clarification qui a émergé parallèlement à la construction des cas durant la phase empirique. S'il s'est révélé étonnamment ardu de comprendre les différentes étapes administratives, leur agencement les unes par rapport aux autres, nous avons constaté que nous partagions l'absence d'une vue d'ensemble avec les personnes candidates, d'une part, et en partie avec les employées et employés en charge des dossiers, d'autre part. Les principes généraux de la procédure sont fixés par les lois (chapitres 3 et 4), mais cette reconstruction porte sur leur mise en application et leur opérationnalisation par les administrations. C'est en organisant les informations et en classant les documents que nous sommes arrivées à la reconstruction schématique qui suit, qui permet de préciser où, et pourquoi, la procédure manque de lisibilité.

Après quelques clarifications terminologiques (5.1), le présent chapitre exposera dans un premier temps, en les commentant, les dispositions pertinentes des différentes lois évoquées et les mettra en regard des informations qui figurent sur les sites officiels (5.2). Ceci nous permettra d'exposer dans un deuxième temps la procédure type, telle qu'elle découle des dispositifs légaux (5.3). Dans un troisième temps, nous allons reconstituer un schéma permettant de visualiser les procédures administratives, schéma général qui sera adapté dans la suite de l'ouvrage aux cas particuliers que nous avons traités (5.4). Enfin, ce chapitre mettra en exergue, sous forme d'excursus, les principaux changements quapportent, depuis le $1^{\text {er }}$ janvier 2018, la révision totale de la loi fédérale sur la nationalité 
ainsi que les révisions correspondantes de la loi cantonale et des ordonnances qui l'accompagnent (5.5).

\subsection{CLARIFICATIONS TERMINOLOGIQUES :}

\section{NATURALISATION ORDINAIRE, FACILITÉE ET SIMPLIFIÉE}

La naturalisation, qui mobilise explicitement l'intégration comme critère d'appréciation de l'aptitude des candidates et des candidats à devenir suisses, est un terme générique qui se décline selon deux volets distincts: la naturalisation dite ordinaire et la naturalisation que la loi fédérale appelle facilitée.

Naturalisation ordinaire et naturalisation facilitée different en termes de conditions et de procédures. La naturalisation facilitée (art. 26 à $32 \mathrm{LN}$ ) est réservée à certaines catégories spécifiques de personnes, essentiellement les conjointes et conjoints ${ }^{1}$ de personnes suisses $^{2}$. Comme elle est traitée presque exclusivement par une autorité fédérale, le Secrétariat d'État aux migrations (SEM), elle est identique pour toute demande, indépendamment du canton de domicile de la personne requérante. En cas de naturalisation facilitée, le canton et la commune sont simplement "consultés» (art. $32 \mathrm{LN}$ ), et aucun des deux n'a de pouvoir décisionnel. Le qualificatif de «facilitée» s'explique par le fait que certaines exigences sont réduites par rapport à la naturalisation ordinaire: la durée de résidence en Suisse n'est que de cinq ans au lieu de douze (art. 27 LN) et les critères liés à l'examen d'aptitude (évaluation de l'intégration) sont allégés ${ }^{3}$. La concentration de la gestion du dossier et du pouvoir décisionnel dans une seule instance, fédérale, et la réduction de certaines exigences formelles rendent la procédure plus simple, souvent plus rapide et moins contraignante que celle de la naturalisation ordinaire, dont relèvent les situations exposées dans le présent ouvrage.

Cependant, dans le langage courant, y compris celui de certains agents administratifs, l'expression "naturalisation facilitée» est polysémique. Le canton de Neuchâtel prévoit en effet une procédure

1. La loi de 1952, en vigueur jusqu'au 31 décembre 2017, exclut les partenaires enregistrés (couples de même sexe) de la naturalisation facilitée, réservée aux couples mariés (hétérosexuels).

2. Pour les quelques autres cas particuliers, voir les articles 29 à $31 b \mathrm{LN}$.

3. Comparer l'art. $26 \mathrm{LN}$, qui demande simplement que la candidate ou le candidat «se soit intégré en Suisse» (let. a), avec l'art. 14, qui requiert, pour la naturalisation ordinaire, que la candidate ou le candidat se soit «intégré dans la communauté suisse» (let. a) et «accoutumé au mode de vie et aux usages suisses» (let. b). Les autres lettres de ces deux dispositions sont pratiquement semblables. 
et des conditions spécifiques pour les «étrangers de deuxième génération $»^{4}$ (art. 11a LCDN), dans laquelle l'évaluation de l'intégration se fait de manière plus ou moins automatique, et la durée de résidence dans le canton est abaissée à deux ans (contre trois dans le cas normal). Il s'agit donc d'une simplification de la procédure administrative et non de la naturalisation elle-même, raison pour laquelle la loi (cantonale) parle de procédure simplifiée. Pourtant, cette procédure simplifiée est parfois qualifiée de "facilitée» par les représentantes et représentants de l'État. Cette similitude terminologique prête à confusion: ainsi, $\mathrm{M}$. Johan, qui a demandé une naturalisation ordinaire en tant qu'étranger de deuxième génération, a reçu un formulaire fédéral intitulé "demande de naturalisation facilitée» (chapitres 2, 6 et 7). L'en-tête de ce formulaire a ensuite été corrigé à la main au sein du service cantonal. Cet exemple de l'emploi d'une même expression pour désigner des procédures différentes accentue encore ce qui nous semble être le manque de lisibilité de ce champ administratif. Dans notre ouvrage, nous utilisons l'expression «naturalisation facilitée» pour désigner la procédure fédérale, et celle de "procédure simplifiée» pour parler de la procédure cantonale neuchâteloise prévue pour les personnes de deuxième génération.

\subsection{LA PROCÉDURE, UN ASSEMBLAGE FÉDÉRAL, CANTONAL ET COMMUNAL}

La reconstruction de la procédure a nécessité de convoquer divers socles légaux. La procédure a été élaborée à partir de la loi fédérale sur l'acquisition et la perte de la nationalité suisse du 29 septembre 1952 (loi sur la nationalité, LN), notamment les articles 12 à 15, qui concernent l'acquisition de la nationalité par voie d'autorités; sur la loi (cantonale) sur le droit de cité neuchâtelois, du 7 novembre 1955 (LDCN); sur l'arrêté du Conseil d'État (neuchâtelois) fixant la procédure d'enquête en matière de naturalisation d'étrangers de la deuxième génération, du 24 février 1999; et sur l'arrêté du Conseil d'État (neuchâtelois) fixant les émoluments prévus par la

4. Pour la définition de ce que signifie deuxième génération, qui peut varier d'un canton à l'autre, voir l'art. $11 a$ al. 3 LDCN, reproduit plus bas, dans le texte (ch. 5.3.2 in fine).

5. La version totalement révisée de cette loi, la nouvelle loi fédérale sur la nationalité suisse (LN), du 20 juin 2014, qui a remplacé l'actuelle loi, dès son entrée en vigueur, le $1^{\text {er }}$ janvier 2018 (Recueil officiel des lois fédérales [RO] 2016 2561-2576), sera toutefois prise en considération de manière ponctuelle. 
loi sur le droit de cité neuchâtelois à percevoir par l'État et les communes, du 6 juillet $2015^{6}$. Notre reconstruction inclut aussi des données produites lors des entretiens et discussions avec les actrices et acteurs administratifs, ainsi que des informations officielles sur les conditions générales de naturalisation publiées sur le site de l'administration cantonale ${ }^{7}$.

La procédure de naturalisation implique les trois niveaux politiques et administratifs de la Suisse, à savoir le niveau fédéral, le niveau cantonal et le niveau communal. La procédure est articulée - voire désarticulée, si nous nous référons au manque de lisibilité de ses étapes - entre ces niveaux, au point que, souvent, ils s'interpénètrent et se chevauchent. Cela complique la compréhension du processus et celle de la répartition des attributions respectives de chacun. Pourtant, les principes déterminés par les textes légaux semblent clairs sur leurs attributions respectives:

a) Les institutions fédérales ont pour attribution, dans le cadre de la loi sur la nationalité, de gérer entièrement la procédure de la naturalisation facilitée (art. 26 à $32 \mathrm{LN}$ ) et, s'agissant de la procédure de naturalisation ordinaire, de vérifier que les personnes candidates à la naturalisation dans un canton et une commune remplissent bien les conditions - minimales - de naturalisation posées par la loi fédérale (art. 14 et $15 \mathrm{LN}$ ). Si tel est le cas, il leur incombe d'accorder l'autorisation fédérale de naturalisation (art. 12 et $13 \mathrm{LN}$ ), par le biais du Secrétariat d'État aux migrations.

b) Une fois accordée l'autorisation fédérale de naturalisation, c'est aux institutions cantonales qu'il appartient d'octroyer la naturalisation cantonale. Pour ce faire, elles s'appuient sur les dispositions du droit cantonal, lesquelles peuvent poser des conditions supplémentaires par rapport au droit fédéral (par exemple, une durée minimale de résidence dans le canton). La décision cantonale implique au préalable l'octroi par la commune du droit de cité communal, puisque, selon la loi fédérale, la «nationalité suisse s'acquiert par la naturalisation dans un canton et une commune» (art. 12 al. $1 \mathrm{LN})$.

6. Tous ces textes, qui ont servi de bases juridiques aux cas concrets sur lesquels portaient notre recherche, sont remplacés, depuis le $1^{\text {er }}$ janvier 2018, par les nouveaux textes, fédéraux et cantonaux, qui sont issus de la révision totale de la loi fédérale sur la nationalité. Ces nouveaux textes légaux, ainsi que les principaux changements qu'ils impliquent, en termes de fond et de procédure, seront présentés plus bas (ch. 5.5, dans le texte).

7. http://www.ne.ch/autorites/DJSC/JUST/naturalisation/Pages/Naturalisationordinaire.aspx (consulté le 15 janvier 2020). 
c) Les institutions communales concourent elles aussi à la naturalisation, dans la procédure de naturalisation ordinaire. Leurs attributions sont réglées par la loi cantonale qui précise leurs tâches et trace les contours de leur marge d'appréciation ${ }^{8}$. À Neuchâtel, les communes ont notamment l'obligation de mettre sur pied une Commission des naturalisations et des agrégations (art. $18 \mathrm{LDCN}$ ). C'est sur la base du préavis de cette commission que le Conseil communal décide de l'octroi ou non du droit de cité communal.

Légalement, la chronologie de la procédure est donc claire: la nationalité suisse s'acquiert par la naturalisation fédérale qui est octroyée si le canton et la commune ont accordé leur droit de cité. Ceci n'est possible qu'à la condition d'avoir préalablement obtenu une autorisation fédérale de l'office fédéral compétent (article 12 al. 1 et 2 LN). Dès lors, nous avions distingué ces deux phases de la manière suivante, qui devait permettre d'expliciter pour chacune d'entre elles les actes administratifs et les institutions concernées:

\begin{tabular}{l|l}
\multicolumn{1}{c|}{$\begin{array}{c}\text { PHASE 1 } \\
\text { FUTORISATION } \\
\text { FÉRELE }\end{array}$} & \multicolumn{1}{c}{$\begin{array}{c}\text { PHASE 2 } \\
\text { OCTROI COMMUNAL } \\
\text { ET CANTONAL }\end{array}$} \\
\hline $\begin{array}{l}\text { Cette première phase nécessite } \\
\text { que la personne requérante remplisse } \\
\text { au préalable un formulaire (fédéral), } \\
\text { disponible auprès des instances } \\
\text { cantonales (JUST et COSM), } \\
\text { et fournisse les pièces justificatives } \\
\text { demandées (attestant de la durée } \\
\text { de résidence en Suisse et de son }\end{array}$ & $\begin{array}{l}\text { Cette deuxième phase est celle } \\
\text { dans laquelle interviennent les } \\
\text { instances décisionnelles qui statuent } \\
\text { explicitement sur l'octroi du droit } \\
\text { de cité communal (Conseil communal, } \\
\text { sur préavis de la Commission des } \\
\text { naturalisations et des agrégations) } \\
\text { et cantonal (Conseil d'État). }\end{array}$
\end{tabular}

intégration). Sur la base de ces

documents, la Confédération, par

le biais du SEM, délivre I'autorisation

fédérale de naturalisation.

8. La loi fédérale, complétée sur ce point en 2007 (entrée en vigueur le $1^{\text {er }}$ janvier 2009) à la suite de plusieurs arrêts du Tribunal fédéral, dont le fameux arrêt Emmen (ATF 129 I 217, A. und Mitb. gegen Einwohnergemeinde Emmen, du 9 juillet 2003), précise que le droit cantonal règle "la procédure aux échelons cantonal et communal» (art. 15a al. $1 \mathrm{LN}$ ). Elle ajoute qu'il "peut prévoir qu'une demande de naturalisation soit soumise au vote de l'assemblée communale», c'est-à-dire au vote du peuple communal (art. $15 a$ al. 2 LN), mais que " $[\mathrm{t}]$ out rejet d'une demande de naturalisation doit être motivé" et qu'une «demande de naturalisation ne peut être rejetée par les électeurs que si elle a fait l'objet d'une proposition de rejet motivée" (art. 15b, al. 1 et 2). Au surplus, les cantons doivent veiller, notamment lorsqu'il y a votation populaire, "à ce que les procédures de naturalisation cantonale et communale n'empiètent pas sur la sphère privée» (art. 15c LN). 
Ce schéma en deux phases nous paraissait à la fois intelligible et cohérent avec les différents textes légaux que nous avons examinés, et nous l'avons utilisé dans un premier temps pour organiser les nombreux documents du dossier de $\mathrm{M}$. Charles, qui nous a servi de cas exemplaire. La tâche paraissait aisée, puisqu'il nous semblait que les documents générés par les instances fédérales correspondaient à la première phase, et ceux estampillés par les administrations cantonales et communales à la seconde. Or, l'étude du dossier de $\mathrm{M}$. Charles a révélé que les différents niveaux politiques et administratifs n'interviennent nullement de manière consécutive, nous forçant à réétudier ce qui se passe dans ces phases.

Le traitement de la demande de $\mathrm{M}$. Charles met en évidence le fait que la première phase de la procédure, dite de demande d'autorisation fédérale préalable à la naturalisation, fait déjà intervenir les trois niveaux administratifs et décisionnels concernés, à savoir la Confédération, le canton et la commune. Il ne s'agit donc pas d'une étape préalable permettant, dans un deuxième temps, le traitement de la demande par les instances décisionnelles (Conseil communal et Conseil d'État). La première phase apparaît, au contraire, comme un moment crucial de la procédure dans lequel sont émis des préavis qui sont souvent réaffirmés dans la même veine (positive ou négative) lors de la deuxième phase. Le cas de $\mathrm{M}$. Charles est particulièrement révélateur à cet égard, puisqu'après une durée de près de dix ans, aucune décision n'avait été rendue durant cette première phase, qui de facto courrait toujours alors même que le dossier avait été archivé (chapitres 6 et 7). Cet état de fait créait une sorte de paralysie institutionnelle, dont la principale caractéristique était de confisquer toute voie de recours au candidat en attente: celui-ci avait été informé par deux courriers que l'autorisation fédérale risquait d'être négative en raison des préavis négatifs de la commune, puis du canton. Ces courriers l'ont amené à contester le bien-fondé de ces préavis auprès de la commune et du canton, conduisant par conséquent l'administration fédérale à différer sa décision formelle dans l'attente de ces précisions. Cette situation particulière, et exceptionnelle, a démontré l'imbrication des niveaux dans les deux phases, ainsi que le manque de lisibilité de la procédure: M. Charles considérait avoir fait deux demandes de naturalisation, et essuyé deux refus de naturalisation, alors que l'administration considérait qu'aucune décision, positive ou négative, n'avait été rendue, le dossier se trouvant toujours dans la 
première phase de demande d'autorisation préalable. La difficulté à démêler cet écheveau complexe était d'autant plus grande que le problème principal rencontré dans la demande d'autorisation fédérale, autorisation qualifiée, rappelons-le, de préalable au traitement cantonal et communal, se situait au niveau de la commune de résidence de $M$. Charles, qui avait émis un préavis négatif.

Pour comprendre la procédure et les imbrications administratives entre les niveaux fédéral, cantonal et communal, il nous a fallu décrire par le menu les différentes étapes et les actrices et acteurs qui y interviennent. La demande d'autorisation fédérale de naturalisation est adressée au Secrétariat d'État aux migrations par le canton, par le biais du Service de la justice, qui a besoin, pour ce faire, de constituer un dossier qualifié de complet par nos interlocutrices et interlocuteurs de l'administration cantonale. Du point de vue légal, c'est l'article $37 \mathrm{LN}$ qui prévoit que «[1]es autorités fédérales peuvent charger l'autorité cantonale de naturalisation d'effectuer les enquêtes nécessaires pour déterminer si le candidat remplit les conditions de la naturalisation".

Le dossier constitué par le canton inclut différents documents dont la nature et la succession sont présentées ci-après, et qui permettent à la commune et au canton de donner un préavis favorable ou défavorable à toute requête. Ces préavis se fondent notamment sur l'examen des aptitudes de la candidate ou du candidat au sens de la loi fédérale sur la nationalité (art. $14 \mathrm{LN}$ ). Cette première phase de l'autorisation fédérale de naturalisation mobilise déjà les trois niveaux administratifs (fédéral, cantonal et communal) sans pour autant que toutes les instances politiques correspondantes (par exemple le Conseil d'État), les seules habilitées à rendre des décisions, ne soient consultées. C'est précisément ce double jeu d'imbrication et de disjonction qui caractérise la procédure. Il y a, d'une part, une imbrication des différents niveaux administratifs lors de la première phase déjà, qui semble relever exclusivement du niveau fédéral. Et, d'autre part, il y a une disjonction entre le rôle de l'administration, responsable de monter le dossier (collecte des documents, enquêtes, etc.), et le rôle des autorités politiques (cantonale et communale), seules aptes à décider, sur cette base, de l'octroi d'un droit de cité. Notre étude montre que cette interpénétration des attributions et des interventions des différents niveaux tout au long de la procédure, et d'entrée de jeu déjà, est un facteur essentiel du manque de lisibilité de la procédure pour les candidates et candidats, voire, 
comme nous l'avons constaté dans certains cas, pour les actrices et acteurs administratifs eux-mêmes, qui ignorent la manière dont se déroule la procédure dans les autres instances concernées.

Une fois l'autorisation fédérale accordée, la procédure cantonale de naturalisation proprement dite peut être initiée, ce qui se fait, à Neuchâtel, par le biais d'une demande de droit de cité dans une commune, sur la base du dossier établi et transmis par le département cantonal compétent (art. 18 LDCN). L'octroi du droit de cité communal permet au canton de statuer sur celui du droit de cité cantonal, compétence du Conseil d'État (art. 22 LDCN).

Ainsi, notre premier constat central est que les trois niveaux politico-administratifs (fédéral, cantonal et communal) ne correspondent pas à des phases successives de la procédure de naturalisation. Au contraire, leurs attributions respectives se retrouvent et s'interpénètrent lors de la première phase déjà, extensive et parfois extrêmement complexe. La seconde phase, celle de la procédure cantonale de l'octroi du droit de cité, fait intervenir les instances décisionnelles tant au niveau cantonal qu'au niveau communal.

\subsection{LES CONDITIONS DE NATURALISATION SELON LES DIFFÉRENTES SOURCES}

Quels rapports entre les conditions légales et la procédure administrative elle-même? Pour répondre à cette question, il s'agit de montrer comment le cadre général de la loi est mis en application par la procédure spécifique que nous avons analysée.

\subsubsection{LA PROCÉDURE D'ACQUISITION DE LA NATIONALITÉ DANS LA LOI FÉDÉRALE}

Dans la loi fédérale, la naturalisation est définie comme l'acquisition de la nationalité par décision de l'autorité (intitulé du chapitre II, qui précède les articles 12 et ss LN). Elle se distingue ainsi de l'acquisition de la nationalité "par effet de la loi» (intitulé du chapitre I de la LN), qui concerne l'acquisition de la nationalité par filiation pour toute personne dont l'un des parents, au moins, est suisse.

La décision de l'autorité en matière de naturalisation combine - et est subordonnée à - deux types de conditions: d'une part, des conditions que l'on peut qualifier de formelles ${ }^{9}$; d'autre

9. L'expression «conditions formelles» apparaît dans la loi de 2014 (art. 9), tout comme l'expression "conditions matérielles" (art. 11). 
part, des conditions matérielles, dont la réalisation suppose une appréciation de la part des personnes en charge des dossiers. Il convient ici de préciser que la notion d'appréciation ne désigne pas un processus subjectif, individuel et discrétionnaire, mais correspond à un ensemble de manières de faire et de critères mis en place à différents échelons de la procédure et de la prise de décision. Parmi les conditions formelles (objectives), figure la durée de la résidence en Suisse, les candidates et les candidats devant prouver, au moyen des attestations de domicile correspondantes, qu'elles ou ils ont "résidé en Suisse pendant douze ans, dont trois au cours des cinq années qui précèdent la requête» (art. 15 al. 1 LN). La loi prévoit également que, dans le calcul de cette durée de résidence, le temps "passé en Suisse entre dix et vingt ans révolus compte double» (art. 15 al. $2 \mathrm{LN})^{10}$. La durée du séjour est établie à partir des attestations délivrées par le contrôle des habitants au niveau communal.

Dans la loi, les conditions matérielles, qui supposent une appréciation, ont trait à l' "aptitude [...] à la naturalisation" des candidates et des candidats. Elles sont prévues à l'art. $14 \mathrm{LN}$, qui exige que la candidate ou le candidat se soit «intégré dans la communauté suisse» (let. a), se soit "accoutumé au mode de vie et aux usages suisses» (let. b), "se conforme à l'ordre juridique suisse" (let. c) et "ne compromet[te] pas la sécurité intérieure ou extérieure de la Suisse» (let. d). Ces conditions sont vérifiées sur la base de données soumises à l'appréciation et à l'évaluation des actrices et acteurs administratifs. Ces données sont élaborées à partir d'enquêtes effectuées par les services administratifs ou sur leur mandat. À Neuchâtel, la police a menée celle sur l'intégration jusqu'en 2010, puis la responsabilité de cette enquête a été transférée au Service (cantonal) de la cohésion multiculturelle (COSM). Les enquêtes comportent aussi des requêtes auprès de tiers (autorités fiscales, par exemple, employeuses et employeurs des candidates et des candidats). Ces vérifications ont pour objectif de déterminer l'attitude des candidates et des candidats au regard des normes légales.

10. En cas de demande collective, la durée de séjour de la conjointe ou du conjoint établi depuis le plus longtemps fait foi (cf. art. 15 al. 3 LN, qui prévoit qu'un séjour de cinq ans, dont l'année qui précède la requête, suffit à l'autre conjoint s'il vit en communauté conjugale avec son conjoint depuis trois ans). Par analogie, cette disposition s'applique aux partenaires enregistrés. 
Pour les procédures initiées dans le canton de Neuchâtel, c'est à l'administration cantonale qu'incombe la vérification des années de résidence. La procédure de naturalisation ordinaire, qui requiert l'obtention du droit de cité dans un canton et une commune (art. 12 al. $1 \mathrm{LN}$ ), ne peut toutefois intervenir que si le Secrétariat d'État aux migrations a accordé l'autorisation fédérale de naturalisation (art. 12 al. 2 LN). Celle-ci n'est valide que dans le canton où la demande initiale est déposée, et pour une période de trois ans, éventuellement prolongeable (art. 13 LN).

\subsubsection{LE PROCESSUS DE NATURALISATION ORDINAIRE À NEUCHÂTEL}

Le site officiel de l'État de Neuchâtel synthétise les conditions posées tant par la loi fédérale que par la loi cantonale, et énonce les étapes générales que suit la procédure ${ }^{11}$. La première d'entre elles consiste à demander, par e-mail, «le formulaire». Sur le site concerné, la nature de ce formulaire n'est pas précisée, mais il s'agit du formulaire de demande d'autorisation fédérale de naturalisation. La demande peut être individuelle ou familiale (regroupant les deux parents, ou l'un d'entre eux et un ou plusieurs de ses enfants).

Le site offre une vision synoptique des conditions nécessaires à remplir pour pouvoir obtenir la naturalisation. Les conditions qui y figurent relèvent toutefois aussi bien de la loi fédérale du 29 septembre 1952 sur l'acquisition et la perte de la nationalité suisse (LN) que de la loi sur le droit de cité du canton de Neuchâtel, du 7 novembre 1955 (LDCN), sans que l'origine de chacune de ces conditions ne soit explicitée. Par ailleurs, les critères spécifiques qui donnent des indications sur l'interprétation des exigences légales n'ont pas pour origine des textes légaux. Par exemple, les critères linguistiques qui figurent dans la liste ci-dessous sont certes fondés sur la LCDN, qui exige que «[p] our acquérir le droit de cité neuchâtelois, la personne qui le demande doit établir: a) qu'elle et ses enfants de plus de seize ans inclus dans l'autorisation fédérale ont des connaissances suffisantes de la langue française» (art. 11 LDCN), mais la loi ne postule pas que ces connaissances suffisantes équivaudraient à l'un ou l'autre niveau, alors que le site mentionne le «minimum de niveau A2 du Portfolio européen des langues (PEL)».

11. http://www.ne.ch/autorites/DJSC/JUST/naturalisation/Pages/Naturalisationordinaire.aspx (consulté le 15 janvier 2020). 
Cette synthèse des conditions posées par le droit fédéral et le droit cantonal telle qu'elle figure sur le site de l'État se présente comme suit (c'est nous qui attribuons l'origine des diverses conditions soit au droit fédéral, soit au droit cantonal, dans la colonne de droite, laquelle ne figure pas sur le site de l'État):

\section{CONDITIONS POSÉES PAR LE DROIT FÉDÉRAL ET CANTONAL}

\begin{tabular}{|c|c|}
\hline $\begin{array}{l}\text { Être intégré•e dans la communauté suisse } \\
\text { - notamment par une intégration sociale et culturelle, } \\
\text { ainsi que par la volonté de participer à la vie économique } \\
\text { ou par la volonté d'acquérir une formation }\end{array}$ & $\begin{array}{l}\text { LN } \\
\text { Manuel du SEM }\end{array}$ \\
\hline Être accoutumé·e au mode de vie et usages suisses & LN \\
\hline Avoir des connaissances suffisantes du français & LDCN \\
\hline $\begin{array}{l}\text { - minimum niveau A2 du Portfolio européen } \\
\text { des langues (PEL) }\end{array}$ & Manuel du SEM \\
\hline Se conformer à l'ordre juridique suisse & LN \\
\hline $\begin{array}{l}\text { - ne pas avoir de poursuites en cours et/ou actes } \\
\text { de défaut de biens ouverts, ainsi que des dettes } \\
\text { envers l'État faisant l'objet d'acte de défaut de biens }\end{array}$ & Manuel du SEM \\
\hline $\begin{array}{l}\text { - être totalement à jour dans le paiement } \\
\text { des contributions fiscales }\end{array}$ & Manuel du SEM \\
\hline $\begin{array}{l}\text { - ne pas avoir d'inscription au casier judiciaire } \\
\text { et/ou d'enquête pénale en cours }\end{array}$ & Manuel du SEM \\
\hline - respecter la Constitution et les lois suisses & Manuel du SEM \\
\hline $\begin{array}{l}\text { Ne pas compromettre la sûreté intérieure } \\
\text { et extérieure de la Suisse }\end{array}$ & LN \\
\hline
\end{tabular}

En dehors des textes légaux, le Manuel du SEM (4.2.3) joue un rôle prépondérant pour encadrer la procédure en donnant une interprétation de critères légaux flous, comme c'est le cas pour comprendre ce que signifient des connaissances linguistiques suffisantes ${ }^{12}$. À ce sujet, le Manuel précise, en page 25, que la personne requérante doit être en mesure de s'exprimer, seule, de manière adéquate au quotidien dans une langue nationale. Les cantons sont libres d'exiger des connaissances de la langue locale, même si, à titre exceptionnel, buergerrecht.html\#Manuel Nationalit\%C3\%A9, (consulté le 15 janvier 2020). 
d'autres éléments peuvent la remplacer ${ }^{13}$. Dès lors, en cas de connaissances très limitées d'une langue nationale, il convient de recueillir des informations complémentaires sur le degré d'intégration si le rapport d'enquête ne contient pas d'indication à ce propos. Le canton de Neuchâtel a concrétisé cet aspect en posant l'exigence du niveau A2.

La procédure de naturalisation ordinaire standard prévoit que la demande de naturalisation neuchâteloise peut être présentée au département compétent une fois l'autorisation fédérale accordée (art. $17 \mathrm{LDCN}$ ). Le département est responsable de compléter au besoin le dossier, puis de le transmettre au Conseil communal (art. 18 LDCN). Celui-ci doit se prononcer dans les trois mois, en fondant sa décision sur le préavis de la Commission communale des naturalisations et des agrégations, une des commissions dont doit obligatoirement se doter chaque commune. Il est intéressant de noter que, dans les termes de la loi, cette Commission communale des naturalisations et des agrégations «organise à son gré l'étude des dossiers qu'elle peut compléter par tous documents utiles» et qu'elle "a en outre la faculté d'entendre toute personne qui est en mesure de la renseigner" (art. 42 al. 1 et 2 LDCN). Elle est par ailleurs « tenue d'inviter le requérant ou la requérante à se prononcer sur les faits relevés à sa charge lorsqu'elle entend proposer le refus de la naturalisation", étant précisé que les "explications sont portées dans le dossier et la commission doit, sur leur vu, se prononcer à nouveau» (art. 44 al. 1 et 2 LDCN).

La commission adresse ensuite "son rapport au Conseil communal en préavisant l'octroi ou le refus de la naturalisation ", la loi prévoyant le contenu minimal de ce rapport: «Le requérant ou la requérante, ainsi que les autres personnes comprises dans la demande, sont désignés par leur nom et tous leurs prénoms. Le rapport indique en outre le pays ou le lieu d'origine, la date et le lieu de naissance, l'état civil, la profession, la durée du domicile en Suisse, dans le canton et la commune» (art. 45 al. 2 LDCN). Une fois que la commune s'est prononcée, elle transmet le dossier et la décision communale au département cantonal compétent en vue de la décision sur la demande de naturalisation cantonale (art. 18 al. 2 LDCN). Celle-ci est de la compétence du Conseil d'État, qui peut charger le département d'une instruction complémentaire (art. $22 \mathrm{LDCN}$ ).

Comme indiqué, la loi cantonale (LDCN) définit également qui sont les étrangères et les étrangers de deuxième génération

13. Arrêt du TAF du 4.11.2008, C-5286/2007, cité par le Manuel du SEM, annexe II, 4.2.1.2. 
pour lesquels la procédure de naturalisation ordinaire est simplifiée. Elle définit aussi les allègements auxquels ces personnes ont droit: les conditions de résidence sont réduites (un séjour dans le canton de deux ans minimum, dont une année dans les deux ans précédant la demande, suffit, contre une résidence dans le canton durant les trois précédant la demande pour les personnes de première génération; art. 11 et $11 a \mathrm{LDCN})$. Aux termes de l'article $11 a$ al. $3 \mathrm{LDCN}$, "[s]ont des étrangers de la deuxième génération les enfants nés en Suisse de parents étrangers ayant immigré, de même que les enfants entrés en Suisse dans la mesure où ils ont accompli dans notre pays la plus grande partie de leur scolarité obligatoire».

C'est au Conseil d'État de fixer les modalités de la procédure d'enquête simplifiée (art. 11a al. 2 LDCN), ce qu'il a fait dans l'arrêté fixant la procédure d'enquête en matière de naturalisation d'étrangers de la deuxième génération, du 24 février 1999 (RSN 131.1). Cet arrêté, très bref, prévoit simplement qu'un questionnaire "visant à compléter les renseignements ressortant de la demande d'autorisation fédérale» remplace le rapport de police (art. 2 al. 1) et que le «service de la justice en détermine le contenu. Au surplus, il recueille, notamment auprès des registres publics, les éléments nécessaires à fonder son appréciation» (art. 2 al. 2).

\subsubsection{COÛTS ET DURÉE DE LA PROCÉDURE}

Ces démarches administratives ont un coût qualifié d'important par les candidates et candidats rencontrés. Il est difficile de donner un montant général, car ce dernier varie en fonction de divers critères: selon les cantons et les communes, qui appliquent des taux variables; selon les administrations nationales d'origine, qui fixent elles-mêmes les prix des documents qu'elles procurent; mais aussi selon la langue des documents officiels étrangers, qui requiert ou non des services de traduction agréés et des procédures d'authentification.

Si l'on reprend ces éléments dans l'ordre, le premier, le plus facile à évaluer de manière chiffrée, est le coût de l'autorisation fédérale de naturalisation, fixé par une ordonnance du Conseil fédéral qui calcule le montant des émoluments perçus par la Confédération ${ }^{14}$ comme suit:

14. L'ordonnance sur les émoluments perçus en application de la loi sur la nationalité, du 23 novembre 2005 (OE-LN, RS 141.21), art. 3 let. a. 
- couple avec ou sans enfants mineurs: 150 fr. ;

- personne seule avec ou sans enfants mineurs: 100 fr. ;

- personne seule mineure: $50 \mathrm{fr}$.

Malgré la révision de 2003 de la loi fédérale sur la nationalité, qui a atténué l'ampleur des fourchettes des émoluments cantonaux et communaux ${ }^{15}$, ces derniers peuvent connaître de grandes amplitudes. Pour Neuchâtel, les émoluments sont fixés par l'arrêté du Conseil d'État du 6 juillet 2015 (RSN 131.21) et ils figurent sur le site ne.ch. Ce dernier mentionne que l'émolument administratif cantonal est calculé par demande, et compris entre $500 \mathrm{fr}$. et 1370 fr., alors que l'émolument administratif communal varie de $100 \mathrm{fr}$. à 150 fr. ${ }^{16}$. Ces estimations manquent quelque peu de précision, puisque l'arrêté fixant les émoluments les détaille de manière précise comme suit:

ACTES

Naturalisation ordinaire

Demande individuelle de personnes

célibataires âgées de moins de 20 ans

Naturalisation ordinaire (1 ${ }^{\text {re }}$ génération)

Demande individuelle avec ou sans enfant

Demande de couple avec ou sans enfant

Naturalisation ordinaire ( $2^{\mathrm{e}}$ génération)

Demande individuelle avec ou sans enfant

Demande de couple avec ou sans enfant

Agrégation

Libération

\begin{tabular}{l|c}
$\begin{array}{c}\text { CANTON } \\
\text { (FR.) }\end{array}$ & $\begin{array}{c}\text { COMMUNE } \\
\text { (FR.) }\end{array}$ \\
600.- & 100.- \\
& $\begin{array}{l}\text { 150.- } \\
\text { 1370.- }\end{array}$ \\
1470.- & $100 . .^{-}$ \\
1220.- & $150 . .^{-}$ \\
1320.- & $300 . .^{-}$ \\
\hline 200.- & \\
\hline $350 .-$ &
\end{tabular}

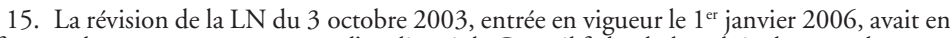
effet pour but, entre autres, comme l'expliquait le Conseil fédéral, de réduire les «émoluments cantonaux et communaux de naturalisation au montant des frais de procédure. Il s'agit en fait de renoncer aux émoluments dits de "rachat" équivalant dans certains cas à plusieurs salaires mensuels. À cause de cette exigence, certaines personnes qui remplissent les conditions de naturalisation doivent y renoncer pour des raisons financières, ce qui est choquant " (Feuille fédérale [FF] 2002 1815-1899, 1817). Le nouvel article 38 al. 1 LN ainsi introduit dispose que « $[1]$ es autorités fédérales, cantonales et communales peuvent percevoir tout au plus des émoluments couvrant les frais pour leurs décisions» (RO 2005 5233-5237).

16. Pour information, le site www.ch.ch indique les moyennes suivantes: au niveau communal, de 500 à 1000 fr. par personne; au niveau cantonal, jusqu’à 2000 fr. par personne. 
Ces frais sont fixes, mais, du côté des administrations suisses, d'autres sont susceptibles de s'y ajouter, en cas d'enquêtes supplémentaires requises par l'une ou l'autre administration, par exemple. De plus, ces émoluments ne comprennent pas le prix d'autres documents officiels à présenter, comme les attestations d'origine, les extraits de casier judiciaire, les extraits de situation fiscale.

Au-delà des documents attestant de la situation administrative en Suisse, les personnes candidates à la naturalisation doivent requérir des actes ou des pièces justificatives auprès des autorités de leur pays d'origine, comme des certificats de naissance, dont le coût varie fortement d'un pays à l'autre. Dans certains cas, ils peuvent être difficiles à obtenir, notamment en cas de conflit actuel ou passé, lorsque la personne fait partie d'un groupe discriminé pour des raisons politiques, ethniques, religieuses ou autres, ou lorsque les conditions nationales d'archivage sont précaires. Enfin, diplômes et certificats établis dans d'autres langues que les langues nationales suisses requièrent des services de traduction agréés, ce qui augmente le budget nécessaire à la constitution d'un dossier. Ces frais, cachés aux yeux des services administratifs helvétiques, n’en sont pas moins réels pour les personnes candidates. À partir du moment où la demande rencontre des obstacles dans son traitement, les coûts augmentent: soit parce que les certificats et documents déjà présentés, qui ont une validité limitée dans le temps, deviennent caducs; soit parce que la personne requérante estime qu'il est préférable de faire recours à une avocate ou à un avocat pour la soutenir dans sa démarche. Ainsi, des démarches comme celles entreprises par $\mathrm{M}^{\mathrm{me}}$ Line et $\mathrm{M}$. Charles (chapitre 7) se chiffrent à plusieurs milliers de francs.

Finalement, les informations fournies par les services administratifs indiquent la durée minimale (dix-huit mois après le dépôt de la demande), mais aucune indication a priori n'est possible quant à la durée maximale, qui dépend de divers acteurs administratifs indépendants les uns des autres. À cet égard, la situation de M. Charles est emblématique, puisque, après près de dix ans, la demande n'a abouti à aucune décision, ni d’octroi ni de refus.

\subsection{SCHÉMAS DE VISUALISATION DES PROCÉDURES}

Le constat du manque de lisibilité, lié à l'imbrication des niveaux fédéral, cantonal et communal dans les deux phases, et de la disjonction entre services administratifs et autorités décisionnelles nous a 
menées à schématiser la procédure sur la base des cas que nous avons analysés. Les schémas qui suivent détaillent les instances impliquées, les documents générés ou rassemblés et les décisions prises. La terminologie qui est utilisée est la suivante:

- Le terme de phase renvoie aux deux moments formellement prévus par la LN, à savoir la demande préalable d'autorisation fédérale (première phase) et l'octroi cantonal et communal du droit de cité (seconde phase);

- Le terme d'étape n'est pas défini de manière aussi formelle. Nous l'utilisons pour rendre compte des différentes démarches des candidates et candidats, des agentes et agents de l'État ainsi que de leurs résultats, sur la base des dossiers que nous avons pu reconstituer et des entretiens.

\subsubsection{PREMIÈRE PHASE: LA DEMANDE D'AUTORISATION FÉDÉRALE}

\section{ÉTAPE 1}

Pour que la procédure puisse être initiée, il faut au préalable qu'une personne devienne candidate à la naturalisation. Pour ce faire, elle doit s'annoncer par e-mail pour solliciter le formulaire de demande d'autorisation fédérale (naturalisation@ne.ch).

En réponse à cet e-mail, elle reçoit des informations générales sur les pièces nécessaires à la constitution du dossier, à savoir les certificats de domicile attestant des années de résidence en Suisse et dans le canton, les copies des documents d'identité (passeport ou carte d'identité, permis de séjour ou d'établissement). Le site ne donne pas de renseignements précis quant aux pièces nécessaires.

Sur la base de ces informations, la personne remplit le formulaire, rassemble les pièces demandées et dépose le tout auprès du Service cantonal de la justice. Nous appelons l'ensemble de ces documents dossier 1 (D1).

\section{RÉCEPTION DU DOSSIER D1 PAR LE SERVICE DE LA JUSTICE}

\section{ÉTAPE 2}

\section{Officiellement}

Transmission de la demande à la Confédération (SEM) par le Service de la justice. Ensuite, le SEM est censé demander au Service de la justice de constituer un dossier.

\section{Dans les faits}

Dans les faits, l'enquête d'intégration est souvent directement demandée à ce moment-là, sans le passage par l'envoi à Berne, qui devrait ensuite renvoyer le dossier à Neuchâtel. II y a donc une contraction des étapes administratives prévues par la loi. 


\section{Enquête d'intégration, ordonnée par le Service cantonal de la justice}

Avant 2009-2010, le mandat d'enquêter était donné aux services de police. À la suite de la modification du règlement d'exécution de la loi sur l'intégration des étrangers (art. 12), effective depuis 2010 pour tout le territoire cantonal, c'est le secteur des naturalisations du COSM qui reçoit ce mandat. Soulignons que l'établissement de rapports d'intégration est une tâche ambiguë pour le $\operatorname{COSM}^{\text {a) }}$. La convention qui régit les relations entre ces deux services précise que le rôle du rapport est de fournir des renseignements permettant de dire si la personne candidate remplit les conditions légales de la naturalisation.

En fonction de chaque profil, le COSM peut demander à la personne requérante, lors de l'entretien, de fournir d'autres documents que ceux déjà déposés en D1, par exemple des attestations de l'office des poursuites, des certificats de travail. Ces documents ne sont pas systématiquement exigés lors du dépôt du dossier D1.

Sur la base de l'entretien, le CosM rédige un rapport, qu'il envoie au Service de la justice, y joignant d'éventuels documents complémentaires.

Le Service de la justice réceptionne ces documents et les joint au dossier D1, qui devient D2.

a) Comme l'atteste un échange de mails (2 octobre 2015) avec une employée qui ne comprenait pas notre question de savoir depuis quand le COSM effectue les entretiens d'intégration. Cette réaction illustre les enjeux liés à la répartition des tâches entre les différents services cantonaux, qui ont des missions distinctes, et se positionnent de manière variable quant à la possibilité et à la pertinence de « mesurer » l'intégration. 


\section{ÉTAPE 3}

\section{Officiellement}

Demande du préavis communal (sur la base du dossier D2), traitée par l'administration communale.

\section{ÉTAPE 4}

\section{Dans les faits}

Dans certains cas analysés, la temporalité successive des demandes présentées par le Service de la justice n'est pas respectée. Certains courriers demandant le préavis communal sont ainsi envoyés à la même date que le courrier demandant au COSM d'établir le rapport d'intégration.

Le Service de la justice réceptionne le préavis communal, le joint au dossier D2, qui devient D3.

Sur la base de ces informations, le Service de la justice peut demander des compléments s'il le juge utile, soit à la personne requérante, soit à d'autres services (service des impôts, police, etc.).

\section{Préavis cantonal émis par le Service de la justice}

Sur la base des documents rassemblés en D3, le Service de la justice donne un préavis, positif ou négatif, sur la possibilité d'octroi du droit de cité cantonal; le préavis est joint au dossier D3, qui devient le dossier D4.

Nota bene: parallèlement à toutes ces étapes, le Service de la justice peut contacter d'autres acteurs institutionnels pour demander des pièces justificatives complémentaires; il peut aussi contacter des personnes citées comme témoins d'intégration par la personne requérante; le cas échéant, au vu des démarches complémentaires entreprises, il peut aussi demander de compléter les émoluments. 


\section{ÉTAPE 5}

\section{Envoi du dossier D4 au SEM par le Service de la justice}

Le dossier D4, qui regroupe les documents rassemblés jusque-là, est envoyé au SEM par le Service (cantonal) de la justice.

\section{Réception du dossier par le SEM}

Prise de connaissance du dossier D4. Le cas échéant, demandes - au canton - de compléments, de clarifications ou de mises à jour. Mais ceci est rare, puisque ne sont envoyés au SEM que les dossiers considérés comme étant «positifs», sur requête de ce dernier (voir chapitre 8).

Après avoir étudié le dossier, le SEM décide de l'octroi de l'autorisation fédérale.

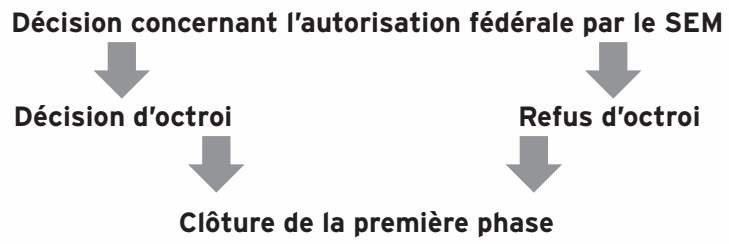

Aussi longtemps qu'aucune décision (octroi ou refus, motivé, de l'octroi) n'est prise, cette première phase n'est pas close. Par conséquent, les candidates et les candidats à la naturalisation ne peuvent faire recours qu'une fois cette cinquième étape achevée (sur les recours, voir ci-dessous).

\subsubsection{SECONDE PHASE: PROCÉDURE DE DEMANDE}

\section{DE DROIT DE CITÉ COMMUNAL ET CANTONAL}

Lorsque le Secrétariat d'État aux migrations octroie l'autorisation fédérale de naturalisation, le dossier aborde la seconde phase de la procédure, qui se déroule auprès des autorités communales et cantonales.

\section{Seconde phase: la demande de droit de cité cantonal et communal}

\section{Envoi du dossier D5 au Service cantonal de la justice par le SEM}

En cas d'octroi de l'autorisation préalable après étude du dossier D4, le SEM renvoie le dossier D5 (D4 auquel est jointe l'autorisation fédérale) au Service cantonal de la justice.

\section{Réception du dossier D5 par le Service cantonal de la justice}

Le Service cantonal de la justice envoie le dossier D5 à la commune; il informe la personne candidate de l'octroi de l'autorisation fédérale. 


\section{ÉTAPE 6}

\section{Traitement par la commune}

L'administration communale reçoit le dossier. Elle le transmet à la Commission communale des naturalisations et des agrégations (formée d'élues et d'élus municipaux) et organise une séance de la commission (ou met le dossier à l'ordre du jour de la séance à venir).

Le dossier D5 est mis à disposition des membres de la Commission communale pour qu'ils en prennent connaissance.

La personne candidate est invitée à venir se présenter à la Commission communale. C'est sur la base de l'étude du dossier D5 et de l'entretien que la Commission communale adresse son préavis au Conseil communal, qui décide d'octroyer ou non le droit de cité communal. Le Conseil communal informe de sa décision le Conseil général.

Nota bene: la décision est très généralement identique au préavis émis lors de l'étape 3 (traité par l'administration communale lors de la phase de demande d'autorisation fédérale). Étant donné que les dossiers perçus comme problématiques, ce qui est le cas des dossiers avec un ou deux préavis négatifs, ne sont pas envoyés au SEM, mais font l'objet de demande de compléments, il est rare qu'il y ait des refus d'octroi à ce stade de la procédure.

La commune joint la décision quant à I'octroi au dossier D5, qui devient D6.

\section{ÉTAPE 7}

\section{Traitement par le canton}

Le dossier D6 revient au Service cantonal de la justice. II est mis à l'ordre du jour de la prochaine séance (bisannuelle) de la Commission chargée de l'examen préalable des dossiers. Ses membres prennent connaissance des résumés de chaque dossier. Le cas échéant, ils discutent de dossiers problématiques, ce qui, en raison de la forme que prend la procédure, est peu fréquent. Cette commission émet une recommandation quant à l'octroi ou non du droit de cité cantonal.

La recommandation est transmise au Conseil d'État neuchâtelois, qui statue sur l'octroi du droit de cité.

Une fois le droit de cité octroyé, le dossier D6 se voit complété par la décision du Conseil d'État, et devient alors D7.

\section{ÉTAPE 8}

\section{Traitement par la Confédération}

Les décisions communale et cantonale sont envoyées au SEM, qui octroie la nationalité suisse en cas de double acceptation. Le document attestant de la naturalisation (acte de naturalisation) de la personne candidate est joint au dossier D7. Ce dernier devient ainsi D8.

La personne candidate est informée de la naturalisation par voie postale.

Le dossier D8 est archivé par le Service cantonal de justice. 


\subsubsection{LES VOIES DE RECOURS}

La procédure prévoit des voies de recours en cas de décision négative de l'une ou l'autre des instances décisionnelles. Ces instances décisionnelles interviennent à l'issue de l'étape 5 (octroi ou refus d'octroi, par le SEM, de l'autorisation fédérale de naturalisation), de l'étape 6 (octroi ou refus d'octroi du droit de cité communal par le Conseil communal) et de l'étape 7 (octroi ou refus d'octroi par le Conseil d'État du droit de cité cantonal).

La décision du SEM de refus de l'autorisation fédérale de naturalisation peut faire l'objet d'un recours au Tribunal administratif fédéral, en vertu des règles ordinaires de la procédure fédérale, alors que les décisions du Conseil communal et du Conseil d'État peuvent, dans le canton de Neuchâtel, faire l'objet d'un recours auprès du Tribunal cantonal ${ }^{17}$. Les décisions du Tribunal cantonal peuvent quant à elles être portées devant le Tribunal fédéral par la voie du recours constitutionnel subsidiaire, pour violation des droits constitutionnels (notamment pour violation de l'interdiction de discrimination).

Il ressort cependant de ces visualisations des diverses phases et étapes de la procédure que si les voies de recours existent, une énorme partie du travail de mise en forme, d'évaluation du dossier et de la personne candidate a lieu en amont, lors de la constitution du dossier par le Service cantonal de la justice - dossier qui est en réalité constitué de plusieurs dossiers successifs par ajout de documents, rapports et préavis. Ces étapes 1 à 4, qui concernent la phase 1 de demande d'autorisation fédérale, voient intervenir administrations communale et cantonale, dont les préavis ont un poids certain pour l'issue de cette phase (obtention ou non de l'autorisation fédérale). Cette imbrication des niveaux administratifs et la multiplication des services cantonaux sollicités contribuent non seulement à rendre la procédure peu lisible, mais constituent aussi des étapes non décisionnelles qui, de facto, ne peuvent faire l'objet de recours, comme nous l'avons montré ci-dessus. Notre observation pose la question de la responsabilité et de sa dilution dans le processus de prise de décision, analysée dans le chapitre 8 .

17. On rappellera que la loi fédérale sur la nationalité, révisée à cet égard en 2007, imposait aux cantons de prévoir une voie de recours auprès d'une autorité judiciaire statuant comme dernière instance cantonale pour les cas de refus de naturalisation ordinaire. Voir, pour le nouveau droit, art. $46 \mathrm{nLN}$. 


\subsection{MISE À JOUR: LA RÉVISION TOTALE DE LA LOI SUR LA NATIONALITÉ}

La loi fédérale sur l'acquisition et la perte de la nationalité suisse, du 29 septembre 1952, qui constituait et constitue la loi applicable aux cas qui ont été étudiés dans notre ouvrage, a fait l'objet d'une révision totale. La nouvelle loi sur la nationalité suisse, du 20 juin $2014(\mathrm{LN})^{18}$, est entrée en vigueur le $1^{\text {er }}$ janvier 2018. Même si cette loi n'était pas applicable au moment où le présent ouvrage a été conçu et rédigé, pas plus qu'elle ne l'est aux cas qui y sont examinés, il convient de la présenter, car les changements qu'elle induit étaient déjà pour certains pris en considération par les agents de l'administration. La loi comporte des changements de fond, d'une part, et de procédure, d'autre part. Nous traiterons, dans un premier temps, de sa genèse, ainsi que des modifications des autres sources légales - fédérales et cantonales - que son adoption a entraînées (5.5.1), puis nous nous pencherons sur les modifications en matière de naturalisation en ce qui concerne le droit de fond, c'est-à-dire les conditions de la naturalisation ordinaire (5.5.2), ainsi que sur les changements de procédure (5.5.3). Nous terminerons par deux compléments concernant des modifications récentes ou en cours (5.5.4).

\subsubsection{GENĖSE}

Après diverses révisions partielles de l'ancienne loi sur la nationalité (en 2003 et 2007 notamment), le Conseil fédéral a proposé, début 2011, une révision totale, qui avait pour buts de simplifier et d'harmoniser les procédures de naturalisation et d'adapter la notion d'intégration exigée par cette loi à celle prévue dans la loi sur les étrangers. Le projet visait, en outre, à limiter l'accès à la naturalisation ordinaire aux personnes titulaires d'un permis d'établissement (permis $\mathrm{C}$ ), ainsi qu'à réduire de douze à huit ans la durée de séjour requise ${ }^{19}$. La discussion de ce projet au Parlement a toutefois pris des directions assez différentes. Le 13 mars 2013, le Conseil national a profondément modifié le projet, en renforçant les exigences de durée de résidence pour la naturalisation ordinaire et en supprimant les facilités de naturalisation pour les jeunes étrangers. Le Conseil des États s'est à son

18. RS 141.0; RO 2016 2561-2576.

19. Message du Conseil fédéral concernant la révision totale de la loi fédérale sur l'acquisition et la perte de la nationalité suisse, du 4 mars 2011, FF 2011 2639-2697. 
tour prononcé sur le projet le 25 septembre 2013, divergeant sur plusieurs points avec le Conseil national. Il estimait, suivant le Conseil fédéral, qu'un séjour en Suisse de huit ans était suffisant pour des personnes étrangères disposant déjà d'un permis $\mathrm{C}$ et il jugeait que les années passées entre 10 et 20 ans devaient encore compter double; il refusait en outre d'admettre le critère d'une "bonne maîtrise" de la langue comme exigence renforcée par rapport à celui de connaissance de la langue qui est déjà prévu parmi les critères d'intégration. Au final, la loi a été adoptée de justesse, le 20 juin 2014, après une longue procédure d'élimination des divergences.

Entrée en vigueur le $1^{\text {er }}$ janvier 2018, la loi est accompagnée, au plan fédéral, d'une nouvelle ordonnance d'application, l'ordonnance sur la nationalité suisse (ordonnance sur la nationalité, OLN), du 17 juin $2016^{20}$. Sur la base de ces nouvelles sources légales, le Secrétariat d'État aux migrations (SEM) a établi de nouvelles directives sur la nationalité (version valable dès le $1^{\text {er }}$ janvier 2018), destinées à encadrer et canaliser l'application de la nouvelle législation par les cantons, qui cohabitent sur le site dudit Secrétariat avec la version valable précédemment ${ }^{21}$.

L'adoption de la nouvelle loi fédérale ${ }^{22}$ et de son ordonnance d'application a entraîné, dans la plupart des cantons, une modification des règles cantonales de mise en ouvre de la législation fédérale en matière de naturalisation. Dans le canton de Neuchâtel, les réglementations légales et réglementaires précédemment examinées - et qui ont constitué les sources normatives applicables aux différents cas examinés dans le présent ouvrage - ont été remplacées par de nouvelles dispositions. L'ancienne loi sur le droit de cité neuchâtelois, du 7 novembre 1955, a été abrogée et remplacée par une nouvelle loi sur le droit de cité neuchâtelois, du 27 mars 2017 (ci-après, nLDCN) ${ }^{23}$, adoptée sur la base d'une proposition du Conseil d'État. Entrée en vigueur le $1^{\text {er }}$ janvier 2018, cette loi cantonale est accompagnée d'un nouveau règlement d'exécution de la loi sur le droit de cité neuchâtelois, adopté par

20. RS 141.01; RO 2016 2577-2592. En vigueur elle aussi depuis le $1^{\text {er }}$ janvier 2018.

21. Voir https://www.sem.admin.ch/sem/fr/home/themen/buergerrecht.html (consulté le 15 janvier 2020), site où les deux Manuels se côtoient.

22. La nouvelle loi porte l'abréviation officielle $\mathrm{LN}$; toutefois, nous l'abrégeons ici nLN, pour la distinguer de l'ancienne loi.

23. RSN 131.0. La nouvelle loi cantonale porte l'abréviation officielle LCDN; toutefois, nous l'abrégeons ici nLCDN, pour la distinguer de l'ancienne loi. 
le Conseil d'État le 3 juillet 201724, qui remplace et abroge, avec effet au $1^{\text {er }}$ janvier 2018, les anciens arrêtés évoqués dans le présent ouvrage $^{25}$.

\subsubsection{DES MODIFICATIONS DE FOND}

Sous l'angle du droit de fond, la nouvelle loi en matière de naturalisation ordinaire prévoit - pour ce qui est des conditions dites «formelles" - que la candidate ou le candidat doit être "titulaire d'une autorisation d'établissement", c'est-à-dire d'un permis C, et apporter la preuve qu'elle ou il «a séjourné en Suisse pendant dix ans en tout, dont trois sur les cinq ans ayant précédé le dépôt de la demande» (art. 9 al. 1, let. a et b nLN) ; dans le calcul de la durée du séjour, «le temps que le requérant a passé en Suisse entre l'âge de 8 et de 18 ans compte double", le "séjour effectif [devant] cependant avoir duré six ans au moins" (art. 9 al. $2 \mathrm{nLN})^{26}$.

Au rang des conditions dites "matérielles", la loi n'innove pas sur le fond, en prévoyant, à l'article 11, que "[l]'autorisation fédérale de naturalisation est octroyée si le requérant remplit les conditions suivantes: a) son intégration est réussie; b) il s’est familiarisé avec les conditions de vie en Suisse; c) il ne met pas en danger la sûreté intérieure ou extérieure de la Suisse». Ces conditions matérielles ne changent donc pas, mais l'article 12 de la nouvelle loi précise, sous l'intitulé "Critères d'intégration", ce qu'il faut entendre par une «intégration réussie», en ces termes:

${ }^{1}$ Une intégration réussie se manifeste en particulier par:

a) le respect de la sécurité et de l'ordre publics ;

b) le respect des valeurs de la Constitution ;

c) l'aptitude à communiquer au quotidien dans une langue nationale, à l'oral et à l'écrit ;

d) la participation à la vie économique ou l'acquisition d'une formation, et

e) l'encouragement et le soutien de l'intégration du conjoint, du partenaire enregistré ou des enfants mineurs sur lesquels est exercée l'autorité parentale.

24. RSN 131.01.

25. Arrêté fixant la procédure d'enquête en matière de naturalisation d'étrangers de la deuxième génération, du 24 février 1999, et arrêté fixant les émoluments..., du 6 juillet 2015.

26. On notera que l'art. $10 \mathrm{nLN}$ prévoit des conditions particulières pour les candidates ou candidats qui ont conclu un partenariat enregistré avec une ou un citoyen suisse. 
${ }^{2}$ La situation des personnes qui, du fait d'un handicap ou d'une maladie ou pour d'autres raisons personnelles majeures, ne remplissent pas ou remplissent difficilement les critères d'intégration prévus à l'al. 1, let. c et d, est prise en compte de manière appropriée.

${ }^{3}$ Les cantons peuvent prévoir d'autres critères d'intégration.

La nouvelle loi neuchâteloise prévoit ainsi, à son article 17, intitulé "Conditions matérielles et critères d'intégration", que:

${ }^{1}$ Le droit de cité cantonal et communal est accordé uniquement si la personne qui le requiert remplit les conditions suivantes:

a) elle satisfait aux conditions matérielles prévues par la loi fédérale pour l'octroi de l'autorisation de naturalisation;

b) elle est apte à communiquer au quotidien dans la langue française, oralement et par écrit ;

c) elle n'est pas défavorablement connue des services de police;

d) elle est, en principe, à jour dans le paiement de ses charges fiscales;

e) elle n'a, en principe, pas de poursuites ouvertes et/ou d'actes de défaut de biens en faveur de la Confédération, du canton et de ses communes.

Le règlement d'exécution précise, s'agissant des «compétences linguistiques» (art. 8), que la "personne qui requiert la naturalisation doit justifier de connaissances orales de la langue française équivalant au moins au niveau B1 du cadre européen commun de référence pour les langues et de compétences écrites du niveau A2 au minimum», et, pour ce qui est de la «situation fiscale» (art. 10), que la "personne qui requiert la naturalisation est à jour dans le paiement de ses charges fiscales, pour autant qu'elle se soit acquittée de l'intégralité des montants facturés échus».

On constate donc, sur la base de ces quelques indications, que les nouvelles réglementations légales comportent, de manière sensiblement plus importante que ce n'était le cas jusqu'ici, des précisions quant à la définition et à la délimitation de la notion d'intégration.

\subsubsection{DES CHANGEMENTS DE PROCÉDURE}

Pour ce qui est de la procédure de naturalisation ordinaire, la nouvelle loi avait pour but, selon le Conseil fédéral, de «réduire les charges administratives des autorités cantonales et fédérales en simplifiant et 
en harmonisant les procédures, de même qu'en clarifiant leurs rôles respectifs en matière de naturalisation ${ }^{27}$. La procédure applicable jusque-là, qui différait fortement d'un canton à l'autre et qui aboutissait parfois à ce que l'autorité fédérale « doive se prononcer sur l'octroi d'une autorisation fédérale bien que le canton ou la commune soit opposé à la naturalisation", est en effet jugée "laborieuse et insatisfaisante». Elle engendre un "travail inutile», qu'il convient d'éviter en prévoyant que «les demandes de naturalisation [soient] déposées auprès d'un service désigné par le canton et [que] les dossiers ne [soient] transmis à la Confédération que lorsque le canton et la commune sont favorables à la naturalisation.» En d'autres termes, il s'agit de renverser la succession des étapes et de donner à l'autorisation fédérale de naturalisation une autre signification: "L'autorisation de naturalisation revêt alors le caractère d'une approbation fédérale de la naturalisation cantonale et communale. ${ }^{28}$

C'est ainsi que la nouvelle loi prévoit que le «canton désigne l'autorité à laquelle la demande de naturalisation doit être adressée» et que, lorsque «le canton et, si le droit cantonal le prévoit, la commune peuvent rendre un préavis favorable quant à l'octroi du droit de cité, ils transmettent la demande de naturalisation au terme de l'examen cantonal au Secrétariat d'État aux migrations (SEM)» (art. 13 al. 1 et 2 nLN). Celui-ci, si "les conditions formelles et matérielles sont remplies, [...] accorde l'autorisation fédérale de naturalisation et la transmet à l'autorité cantonale, qui rend la décision de naturalisation" (art. 13 al. 3). C'est donc ensuite l'autorité cantonale compétente - à Neuchâtel, le Conseil d'État ${ }^{29}$ - qui "rend la décision de naturalisation dans le délai d'un an à compter de l'octroi de l'autorisation fédérale» (art. 14 al. $1 \mathrm{nLN}^{30}$ ), le «droit de cité communal et cantonal et la nationalité suisse [étant] acquis lors de l'entrée en force de la décision cantonale de naturalisation" (art. 14 al. 3 nLN).

La loi fédérale précise encore que le «droit cantonal régit la procédure aux échelons cantonal et communal» et qu'il "peut prévoir qu'une demande de naturalisation soit soumise au vote de

27. Message du Conseil fédéral, précité, FF 2011 2639-2697, 2640.

28. Sur cette appréciation de la procédure applicable jusqu'alors et ces changements proposés, Message du Conseil fédéral, précité, FF 2011 2639-2697, 2643-2644.

29. Art. 4 al. 1 let. a nLDCN.

30. L'alinéa 1 précise que, passé ce délai, cette autorisation échoit. L'alinéa 2 précise pour sa part que l'autorité cantonale "refuse la naturalisation si, après l'octroi de l'autorisation fédérale, elle apprend des faits qui l'auraient empêchée de rendre un préavis favorable quant au droit de cité». 
l'assemblée communale» (art. 15 al. 1 et $2 \mathrm{nLN}$ ), tout rejet d'une demande de naturalisation devant être motivé (art. 16) et les procédures, communale et cantonale, devant respecter la sphère privée des candidates et candidats (art. 17). Enfin, autre nouveauté, la loi sur la nationalité ajoute, sous l'intitulé «Durée de séjour cantonal et communal», que la "législation cantonale prévoit une durée de séjour minimale de deux à cinq ans» (art. 18) ${ }^{31}$.

Sur la base de cette réorientation de la procédure imposée par le droit fédéral, la nouvelle loi sur le droit de cité neuchâtelois règle la procédure cantonale et communale de manière différente de ce qu'elle était jusqu'ici. L'art. $18 \mathrm{nLDCN}$ prévoit que la « demande est déposée auprès du service [de la justice ${ }^{32}$ ] au moyen du formulaire officiel de demande d'autorisation fédérale de naturalisation complété par les documents désignés dans le règlement d'exécution". L'art. 11 dudit règlement énumère de manière détaillée les différents documents à fournir. L'art. $19 \mathrm{nLDCN}$ précise que la demande n'est traitée que si la personne candidate s'acquitte préalablement de l'émolument cantonal ${ }^{33}$; à défaut, sa demande est déclarée irrecevable. L'art. 20 précise en outre que si les conditions formelles ne sont pas remplies et si la demande n'est pas déposée conformément à l'art. 18, le service rend une décision de classement.

Dans les autres hypothèses, le "service effectue les enquêtes nécessaires pour déterminer si les conditions matérielles et les critères d'intégration sont remplis» (art. 21 al. 1 nLDCN). Il «peut déléguer entièrement ou en partie sa compétence d'effectuer les enquêtes» (art. 21 al. 2). Une fois admis que les conditions matérielles et les critères d'intégration sont remplis, «le service transmet le dossier au conseil communal de la commune dans laquelle est domiciliée la personne qui requiert la naturalisation au moment du dépôt de la demande» (art. 21 al. 3) (34 $^{34}$

L'art. $22 \mathrm{nLDCN}$, qui régit la procédure de la décision communale, prévoit que le «conseil communal complète au besoin

31. Le canton de Neuchâtel a choisi la durée minimale la plus courte: la personne candidate doit, au dépôt de la demande, être "domiciliée dans le canton depuis deux ans" (art. 14 let. b nLDCN).

32. Art. 5 du règlement d'exécution de la loi cantonale.

33. Voir l'art. 16 du règlement d'exécution de la loi, qui fixe les différents émoluments, alors que l'art. $13 \mathrm{al}$. 1 prévoit que l'«émolument cantonal est perçu en totalité au moment du dépôt de la demande de naturalisation".

34. La loi précise que, dans «le cas où les conditions matérielles et les critères d'intégration ne sont pas remplis, le Conseil d'État rend une décision de refus de naturalisation, sur préavis de la Commission cantonale des naturalisations» (art. 21 al. 4 nLDCN). 
le dossier et statue dans un délai de trois mois sur préavis de la commission communale des naturalisations et des agrégations" (al. 1). Le dossier est ensuite "retourné au service [cantonal], accompagné de la décision communale» (art. 23 al. 1 nLDCN) et, " [s]i le droit de cité communal est accordé, le service transmet la demande à l'autorité fédérale avec un préavis favorable à l'octroi du droit de cité cantonal» (art. 23 al. 2 nLDCN); "[s]i le droit de cité communal ou l'autorisation fédérale n'est pas accordé, le service rend une décision de classement» (art. 23 al. 3 nLDCN).

Enfin, une fois que «l'autorisation fédérale est accordée, le Conseil d'État statue sur préavis de la commission cantonale des naturalisations» (art. 24 al. 1), la loi précisant que la «naturalisation est refusée par le Conseil d'État uniquement s'il apprend des faits nouveaux qui auraient empêché un préavis favorable, selon l'article 23, alinéa 2 ».

Les révisions totales des lois fédérale et cantonale apportent à la procédure schématisée ci-dessus - telle qu'elle se présentait dans les différentes situations étudiées dans le cadre de notre projet - des modifications sensibles. Ces nouvelles législations, c'est du moins leur objectif et leur ambition, devraient simplifier la procédure et ses diverses étapes et, il faut l'espérer, en améliorer la lisibilité, pour les observatrices et observateurs tiers, tels que nous, mais aussi pour les actrices et acteurs concernés eux-mêmes, que ce soit du côté de l'administration ou de celui des personnes candidates.

\subsubsection{COMPLÉMENTS}

Il convient encore de mentionner deux compléments, concernant, respectivement, la naturalisation facilitée des étrangers de la troisième génération et le renforcement de l'intégration des étrangers.

Avant même son entrée en vigueur, la nouvelle loi sur la nationalité a fait l'objet d'une première révision partielle, à la suite de la votation populaire du 12 février 2017. Ce jour-là, en effet, le peuple et les cantons ont accepté l'arrêté fédéral du 30 septembre 2016 portant modification de l'article 38 al. 3 de la Constitution fédérale et prévoyant que la Confédération facilite la naturalisation "des étrangers de la troisième génération" (let. a), ainsi que "des enfants apatrides» (let. b). Adoptée par le Parlement le 30 septembre 2016, en même temps que l'arrêté constitutionnel, la modification correspondante de la loi sur la nationalité du 20 juin 2014 concrétise cette ouverture de la naturalisation facilitée aux 
étrangers de la troisième génération. Le nouvel article $24 a$ de la loi prévoit ainsi que l'enfant de parents étrangers peut, sur demande (déposée avant l'âge de 25 ans révolus), obtenir la naturalisation facilitée si plusieurs conditions sont réunies: "a. l'un de ses grands-parents au moins est né en Suisse ou il peut être établi de manière vraisemblable que celui-ci a acquis un droit de séjour en Suisse; b. l'un de ses parents au moins a acquis une autorisation d'établissement, a séjourné en Suisse pendant au moins 10 ans et a accompli au moins 5 ans de scolarité obligatoire en Suisse ; c. il est né en Suisse; d. il est titulaire d'une autorisation d'établissement et a accompli au moins 5 ans de scolarité obligatoire en Suisse. " ${ }^{35}$

Parallèlement à la nouvelle loi sur la nationalité, les Chambres fédérales ont adopté, en date du 16 décembre 2016, une révision partielle de la loi sur les étrangers portant principalement sur les dispositions de cette loi qui traitent de l'intégration des personnes étrangères en Suisse ${ }^{36}$. Fondée sur deux messages du Conseil fédéral de 2013 et de $2016^{37}$, cette révision avait en particulier pour but, en s'appuyant "sur les dispositions en vigueur en matière d'intégration, selon le principe éprouvé "encourager et exiger" ", de "rendre l'intégration des étrangers plus contraignante» et de faire ainsi en sorte que ceux-ci «engagent davantage leur responsabilité individuelle en vue de s'intégrer ${ }^{38}$. La nouvelle loi, entrée en vigueur le $1^{\text {er }}$ juillet 2018, renforce les exigences en matière d'intégration pour les personnes étrangères qui sollicitent un permis d'établissement en Suisse et porte désormais le titre de loi fédérale sur les étrangers et l'intégration (LEI), ce qui symbolise l'importance que le législateur accorde à cette notion d'intégration.

\subsection{SYNTHÈSE: UNE ENTREPRISE DE CLARIFICATION}

Cette reconstitution de la procédure et de ses spécificités a permis de mettre en évidence les mécanismes qui ont contribué à rendre la trajectoire des dossiers opaque. Elle met en évidence plusieurs niveaux d'imbrication qui rendent difficilement lisible, pour les

35. RO 2017 2643-2644, pour l'arrêté constitutionnel, et FF 2017 3197, pour la révision de la loi, dont l'entrée en vigueur coïncide avec celle de la nouvelle loi sur la nationalité, soit le $1^{\text {er }}$ janvier 2018.

36. FF $20168633-8650$, pour le texte de la loi.

37. Message relatif à la modification de la loi fédérale sur les étrangers (intégration), du 8 mars 2013, FF 2013 2131-2190, et Message additionnel concernant la modification de la loi fédérale sur les étrangers (intégration), du 4 mars 2016, FF 2016 2665-2704.

38. Message de 2013, précité, FF 2013 2131-2190, spéc. 2669. 
personnes candidates, ce qui se passe lors du traitement de leur dossier.

En premier lieu, nous avons souligné les ambiguïtés terminologiques: l'utilisation, dans le langage administratif courant, de l'expression "naturalisation facilitée " pour se référer à la procédure simplifiée de la naturalisation ordinaire, cantonale, et non à la procédure facilitée, fédérale, non décrite ici, illustre le flou qui entoure la procédure.

En second lieu, la répartition des tâches entre les divers acteurs institutionnels lors des deux phases prête à confusion. La première phase, qui correspond à la demande de l'autorisation fédérale, semble a priori une phase routinière. Dans les faits, c'est à ce moment que les pièces maîtresses du dossier final sont élaborées, notamment le rapport d'intégration, qui, on le verra dans les chapitres 6,7 et 8 , joue un rôle central. Or, les instances décisionnelles n'interviennent pas, ou très peu, durant cette phase purement administrative. Cela indique, et en même temps explique, le poids prépondérant des services administratifs qui constituent et traitent le dossier lors des diverses étapes. Ceci est d'autant plus marquant lors de contractions des étapes censées se dérouler de manière chronologique, mais qui, parfois, se déroulent simultanément (cf. étapes 2 et 3). Nous le montrerons dans les chapitres qui suivent, le manque de lisibilité a priori de la procédure quant à sa durée, à ses coûts et aux mécanismes administratifs et décisionnels qui la composent a des effets concrets sur les personnes candidates à la naturalisation. 


\section{PARTIE III}

ANALYSE DES TRAJECTOIRES 



\section{FAIRE L'EXPÉRIENCE \\ DES PROCÉDURES: \\ TRA JECTOIRES DE VIE}

TANIA ZITTOUN

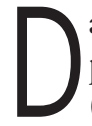

ans ce chapitre, nous analysons les trajectoires de vie des personnes. Pour reprendre notre construction théorique (chapitre 1), nous nous centrons ici sur la ligne du bas, qui regroupe les sphères d'expérience de la personne qui constituent sa trajectoire de vie. Nous commençons par rappeler quelques éléments théoriques et méthodologiques permettant de comprendre, d'un point de vue psychologique, certains des processus par lesquels les personnes peuvent développer leur trajectoire de vie unique, ce qui peut menacer cette dernière, et ce qui peut la soutenir (6.1). Sur cette base, nous nous penchons sur les cas des personnes dont nous avons analysé les dossiers et/ou les récits. Nous nous focalisons sur ce que nous savons de leur trajectoire de vie en lien avec la procédure administrative et légale liée à la naturalisation ou à la prolongation de permis, mais aussi au-delà de cette procédure. Nous présentons une étude de cas exemplaire, celle de M. Charles (6.2), avant d'adopter une lecture plus transversale des autres cas (6.3) et de proposer un premier bilan du rôle transformatif des trajectoires administrativo-légales dans la trajectoire de vie des personnes (6.4).

\subsection{PENSER ET ANALYSER LES TRAJECTOIRES DE VIE}

Cette première section rappelle la spécificité d'une psychologie socioculturelle du cours de la vie (voir aussi chapitre 1) en mettant en évidence les enjeux subjectifs de l'expérience de migration ou plus généralement de la confrontation à des situations nouvelles (6.1.1). Sur cette base, elle décrit la méthodologie qualitative et les étapes de l'analyse qui nous a permis de reconstituer les cas que nous décrivons dans ce chapitre (6.1.2). 


\subsubsection{THÉORISER LE COURS DE LA VIE ET L'IMAGINATION}

Selon la perspective socioculturelle adoptée ici, la trajectoire de vie de la personne peut être considérée comme étant constituée d'une pluralité de «sphères d'expérience». Une sphère d'expérience est une configuration d'activités, de sentiments, de relations, d'émotions et d'engagements identitaires suffisamment récurrents, ou réguliers, pour que la personne les considère comme étant du même type: manger en famille, travailler, faire de la musique avec des amis, etc. Au quotidien, les personnes alternent ces sphères d'expérience, qui font ainsi partie de ce qui va de soi pour elles. Les personnes peuvent également parfois faire l'expérience de ruptures, d'événements ou de situations qui remettent en question cet allant de soi, et qui demandent que, face à de nouvelles situations, elles changent, qu'elles inventent de nouvelles manières de faire, de comprendre, de s'exprimer ou de se définir, créant ainsi de nouvelles sphères d'expérience. Nous avons appelé transitions les processus par lesquels les personnes engagent ainsi des transformations identitaires, de nouveaux apprentissages ou donnent sens à de nouvelles situations ${ }^{1}$. Une trajectoire de vie est ainsi constituée de différentes sphères d'expérience et de transitions douces quotidiennes, ponctuées par des expériences de ruptures qui peuvent au contraire engager des transitions plus radicales; la migration, typiquement, peut générer des ruptures dans de nombreuses sphères d'expérience.

La psychologie du développement, inspirée par la psychologie clinique et par l'étude de biographies, a mis en évidence le fait que, lorsque les personnes vivent des changements ou des ruptures, elles ont besoin de maintenir un sens fondamental de leur propre continuité et de leur intégrité 2 . La notion de continuité désigne l'expérience de la personne d'être encore la même, c'est-à-dire de pouvoir se reconnaître, ou du moins de pouvoir lier, d'une manière ou d'une autre, son présent avec son passé. La notion d'intégrité renvoie quant à elle au fait de respecter ce que la personne considère comme faisant partie de ce qui fait son identité ou de ce que William James appelle son Soi étendu ${ }^{3}$; elle peut inclure le fait de ne pas vivre des expériences qui sont trop contraires à l'idée que la

1. Perret-Clermont et Zittoun, 2002; Zittoun, 2012; Zittoun et Gillespie, 2015b, 2015a; Zittoun et Perret-Clermont, 2009.

2. Erikson, 1959, 1993.

3. Goffman, 1997; James, 2007. 
personne se fait d'elle-même ou qui affectent son sentiment d'intégrité psychique, morale ou physique. Cela peut aller des besoins de reconnaissance sociale et symbolique au fait de pouvoir conserver des objets qui peuvent avoir une valeur importante pour soi. Enfin, les personnes ont besoin de sentir que «la vie vaut la peine d'être vécue», ce que Winnicott ${ }^{4}$ désigne par le terme de créativité existentielle. Nous avons étendu cette idée à la proposition selon laquelle les personnes engagent, dans leur vie quotidienne, leur imagination, qui leur permet à la fois de revisiter leur passé, d'imaginer des alternatives ou de créer des futurs possibles. Manifestation de la vie psychique, nourrie par l'environnement socioculturel, l'imagination est aussi le processus par lequel la personne peut se dégager, au moins temporairement, des contraintes de l'ici et maintenant et, éventuellement, engager des actions qui peuvent lui permettre de modifier des situations quotidiennes et sa trajectoire de vie ${ }^{5}$. À la lumière de ces éléments, nous nous demandons en quoi les démarches administratives qu'initient les personnes affectent leur trajectoire de vie. Quelles sont les sphères d'expérience concernées par ces démarches? Comment les personnes donnent-elles sens à ces démarches? Celles-ci ont-elles des répercussions sur d'autres sphères d'expérience? En quoi ces démarches créent-elles des ruptures ou remettent-elles en question le sentiment de continuité ou d'intégrité de la personne? A quelles conditions les personnes peuvent-elles néanmoins maintenir une imagination de leur avenir? Si, nous l'avons vu, la loi fait de la procédure de naturalisation une mesure visant à l'intégration, est-ce bien ce que les personnes vivent?

\subsubsection{ANALYSER DES TRAJECTOIRES DE VIE}

Pour rendre compte de l'expérience des personnes, nous nous fondons ici sur les discours, oraux et écrits - entretiens et documents écrits (lettres, formulaires) - produits par et au sujet des cinq personnes dont nous avons reconstruit les dossiers $\left(\mathrm{M}^{\mathrm{me}}\right.$ Line et MM. Charles, François, Johan et Kirin, voir chapitre 2). Nous faisons en plus appel aux entretiens effectués dans le cadre de ce projet avec quatre autres personnes engagées dans des procédures de naturalisation ou de prolongation de permis (MM. Armand et Bruno, et $\mathrm{M}^{\mathrm{mes}}$ Dominique et Elvire). En effet, les difficultés

4. Winnicott, 2002.

5. Zittoun et de Saint-Laurent, 2015; Zittoun et Gillespie, 2016. 
rencontrées par ces dernières permettent de mettre en lumière certains aspects mentionnés par les premières.

Les entretiens et les cas reconstitués (chapitre 2) ont été analysés qualitativement en faisant appel au logiciel Atlas-ti, qui permet de coder les discours et d'organiser ces codes de différentes manières. Nous avons été attentives aux aspects relevant de la procédure: les justifications de la procédure et les arguments donnés par les personnes de l'administration, rédigés ou rapportés oralement. Nous avons également codé des catégories émergentes (par exemple sur l'expérience de racisme quotidien, ou relatives au sentiment d'être inconnu), parfois recodées à partir de la littérature (par exemple en expérience de non-reconnaissance). Nous avons enfin créé des catégories à partir de notre cadre théorique, nous invitant par exemple à identifier les différentes sphères d'expérience de la personne, ses relectures du passé ou son imagination d'avenirs possibles. Quand cela sera nécessaire, nous ferons appel à la littérature disponible pour donner du relief à nos analyses.

Avant de donner une vue d'ensemble de la manière dont les trajectoires administratives des personnes participent à leurs trajectoires biographiques, nous proposons d'abord une étude de cas; elle nous servira de contrepoint aux autres situations, que nous analyserons de manière plus transversale.

\subsection{UN CAS EXEMPLAIRE: M. CHARLES}

Le cas de M. Charles peut être considéré comme exemplaire ${ }^{6}$, dans le sens où la plupart des éléments que nous avons identifiés dans les autres cas s'y trouvent réunis. Nous avons également son dossier complet. En outre, M. Charles s'exprime relativement facilement et ouvertement en français; son récit biographique constitue donc une source intéressante de données.

Nous mettons ici en évidence la trajectoire biographique de M. Charles, ainsi que son lien avec sa trajectoire administrative. Plus exactement, nous mettons en évidence ce que nous savons de sa vie avant le dépôt de sa demande de naturalisation (6.2.1), et la manière dont il formule celle-ci (6.2.2); nous examinons ensuite la façon dont il comprend le travail de l'administration (6.2.3) et le refus qui lui sera adressé (6.2.4); nous montrons enfin plus

\section{Flyvbjerg, 2011.}


globalement la manière dont cette procédure participe à la transformation de sa trajectoire biographique (6.2.5). Dans les chapitres suivants, nous reviendrons en outre sur l'agentivité de $\mathrm{M}$. Charles et des autres personnes (chapitre 7), et sur le travail administratif à l'œuvre dans ce cas comme dans d'autres (chapitre 8).

\subsubsection{AVANT LA DEMANDE: RUPTURES ET TRANSITIONS}

M. Charles est arrivé en Suisse adolescent pour fuir un pays dans lequel son père et sa famille étaient victimes de violences politiques. Voilà la manière dont il en parle lors de l'entretien de recherche que nous avons effectué en juin $2014^{7}$ - sachant qu'il a eu l'occasion de rédiger sa biographie lors d'un stage d'aide à l'orientation professionnelle et probablement de la raconter plusieurs fois :

Il y avait eu un renversement du gouvernement, tout ça, puis il $\mathrm{y}$ avait des discriminations sur la population parce que nous, nous sommes de [A]. [...] Il y a eu beaucoup de massacres, beaucoup de discriminations, beaucoup d'interdictions sur... Au niveau culturel, religieux... Tous ces aspects-là. Et donc, [dans un] premier temps, c'est mon père qui a quitté [le pays]. [...] Puis nous, on est restés pendant sept ans en fait, sans voir notre père, avec mes [frères et sœurs]. [...] On était dans un petit village, après on a déménagé dans une ville pour étudier, puis justement moi j'ai étudié là-bas, j’ai fait mon école, lycée, j'ai fini le lycée, en deuxième année j'ai fini, en troisième je n'ai pas pu finir, et puis on a aussi été obligés de quitter le pays aussi. Je n’ai pas pu finir mes études. [...] C'est pour raisons politiques, parce que ça continuait, ça n'arrêtait pas. Il y a eu, tout d'abord mon oncle qui faisait partie de... Membre d'un parti politique de... illégal [dans le pays] [...]. Ils l'ont pris, puis on était surveillés par la police, tout ça, etc. Et lui ils l'ont pris, ils l'ont mis en prison, ils lui ont fait beaucoup de torture, machin et tout. Et après ils l'ont mis en prison, il est resté quand même quelques années. Puis il a pris une occasion, il s'est enfui. [...] En fait la pression s'était encore renforcée... [...] Contre la famille encore. Il y avait sur la population de toute façon, mais quand y a encore des gens qui connaissaient encore plus la famille, ils ont insisté, ils attaquaient encore plus. Et nous,

7. Les extraits d'entretien avec M. Charles sont tous tirés de cet entretien ; les sources des autres extraits sont indiquées. 
y a eu justement... C'était impossible de rester là-bas. [...] Ils surveillaient tout ce qu'on faisait, ils posaient les questions, avec des opérations pendant la nuit, avec des militaires, minuit, etc. On était petits tout ça... [...] J'[avais] encore 17 ans. Puis quand j'ai eu tout ça, voilà, je me rappelle, j'avais l'âge de 14-15 ans, je me rappelle qu'on voyait, on recevait à la maison à minuit, tout ça, il y avait des opérations militaires au village, ou dans la maison, etc. [...] C'était menaçant. Parce qu'il y a eu des massacres, puis il y a eu beaucoup de gens qui ont perdu leur vie à cette époque. Mon oncle c'était tout juste tout juste, il a... Parce qu'ils lui ont dit "enfuis-toi », il a essayé de fuir, ils lui ont tiré derrière! Il n’a pas... Il ne s'est pas enfui... il n'est pas parti, ils ont voulu le piéger, tout ça. Puis à la fin ils l'ont mis en prison, il s'est enfui en prison, il a eu l'occasion de partir, fuir en prison. En fait voilà, c'est ces raisons-là qui nous ont amenés à quitter notre pays natal.

M. Charles évoque aussi le voyage difficile qui a mené la famille de son pays à la Suisse pour rejoindre le père de famille:

Et puis y a une autre souffrance, le chemin! Pour arriver en Suisse, ça faisait trois mois. Donc on est sortis [du pays], c'était fin août, début septembre. Et puis on est arrivés au mois d'avril en Suisse. Donc pendant trois mois on était en chemin. On devait passer toutes les frontières en Europe pour arriver en Suisse. [...] Pendant trois mois donc on a, donc mon père il nous a envoyé de l'argent pour trouver [des] faux passeports, y a les vrais passeports et les faux passeports. Parce qu'avec le vrai passeport on a passé la frontière de [notre pays], où ils ne demandent pas les visas. Après, le pays qui demandait le visa, on avait des faux passeports et des faux visas. Donc tous ces passeports faux on a trouvé [dans notre pays]. Et pour utiliser après. Et nous on est parti... On s'est coincés là-bas [dans le pays voisin]. Avec faux passeports. On n'a pas pu passer aucun pays où on pouvait entrer parce qu'ils l'ont reçu, et on s'est coincés là-bas. Et puis on n'avait pas de moyens pour ni retourner, ni pour avancer. On était coincés là-bas! Et puis ils nous ont... Il y avait une personne de quelqu'un qui nous a fourni les passeports. Il nous a fauché un peu de l'argent, il nous a presque... plus d'argent. Ils nous ont mis dans un hôtel et puis il s'est cassé avec la moitié de notre argent, il nous a laissés là-bas, il s'est cassé. Pis nous, on ne savait pas la langue, on était [dans ce pays voisin]. 
On est resté pendant vingt jours, on était coincés là-bas. On ne savait pas la langue, on ne savait pas l'écriture [...], on ne savait pas le chemin, on était coincés là-bas en fait! [...] Et puis mon père il a envoyé quelqu'un depuis ici. Un de ses camarades. Parce que mon père $[\ldots]$ il faisait des activités culturelles, politiques ici à cette époque-là [en Suisse]. Et mon père il a envoyé un de ses camarades pour nous sortir de cette histoire-là. [...] Après on a réfléchi à qu'est-ce qu'on pouvait faire, où on pouvait aller avec ces passeports. On a avancé par [un deuxième pays européen] en train. Ils nous ont arrêtés là-bas. Après avec les négociations encore [suit un voyage qui passe par trois autres pays européens avec des allers-retours]. Cette fois-ci on était coincés [dans un troisième pays], on a essayé en car, pour passer [dans un pays limitrophe de la Suisse], ils nous ont laissés, mais cette fois-ci c'était celui qui nous accompagnait il avait quelque chose sur son passeport qui ne jouait pas. À cause lui on n'a pas pu rentrer, on a dit bon, on essaie la prochaine fois et puis lui il a essayé de faire à jour son passeport. On a réessayé une deuxième fois, cette fois-ci c'était nous que ça n'a pas marché. On est à nouveau retournés [dans le troisième pays]. Après on a trouvé aussi des gens [...] qui faisaient des passages [de] frontière, on a trouvé, on a rencontré des gens qui faisaient passer les gens. Puis on a passé depuis [ce pays] par [un deuxième pays limitrophe de la Suisse], on est rentrés en Suisse. Le chemin s'est passé comme ça.

Nous présentons ces deux longs extraits pour préserver l'oralité du récit, qui contraste avec le style d'élocution de $M$. Charles ailleurs dans l'entretien. Après une première version plus narrativisée des difficultés de la famille, M. Charles semble ici se remémorer certaines des scènes de son enfance, comme le suggèrent des formules comme "Voilà, je me rappelle, j'avais l'âge de 14-15 ans, je me rappelle qu'on voyait...». Elles surgissent avec leurs émotions (ce sont des remémorations vitales) ${ }^{8}$; encore peu distancées et élaborées, elles produisent ce discours haché, qui superpose des expériences intenses. Le récit du voyage en Europe, qui avait pourtant déjà fait l'objet de remémorations, arrive aussi comme une longue succession d'événements peu connectés les uns avec les autres, probablement encore revus avec le regard d'un adolescent ${ }^{9}$. Sans entrer

8. Brown et Reavey, 2015.

9. Bion, 1989; Brown et Reavey, 2015. 
dans une analyse plus fine, on peut donc retenir que $M$. Charles a vécu des événements d'une grande intensité émotionnelle avant et jusqu'à son arrivée en Suisse, qui vraisemblablement ont provoqué des expériences de rupture assez radicales. Les souvenirs de ces événements datant d'une vingtaine d'années sont encore vifs, comme on en sent les traces dans le discours.

Après cet épisode confus et probablement difficile, la famille est accueillie dans un camp de réfugiés, puis parvient à rejoindre le père en Suisse romande. La famille obtient le permis B puis le C. M. Charles, alors âgé de 17 ans, est admis en classe d'accueil où il reste presque deux ans. Il a la possibilité de commencer un apprentissage, mais le quitte pour prendre un emploi ouvrier. Il est à noter que $\mathrm{M}$. Charles avait fréquenté le lycée dans son pays d'origine et la famille avait espéré partir après qu'il eut obtenu un diplôme en lettres et sciences humaines, ce qui suggère qu'elle avait des aspirations pour lui; ces études ne sont toutefois pas reconnues en Suisse, et il ne peut donc pas poursuivre la filière débutée. Pour des raisons linguistiques, il est orienté vers des filières techniques. Ainsi, si la migration appelle de toute façon de nouveaux apprentissages (linguistiques, etc.), elle demande aussi ici une transformation identitaire et un changement de projets pour soi ${ }^{10}$. De plus, M. Charles semble avoir pris un emploi salarié en raison des dettes de la famille, acquises lors du voyage qui l'a menée en Suisse:

C'était très très dur aussi, mon père il avait des dettes parce qu'il avait, il devait de l'argent à ses amis, ses copains, parce qu'il nous a envoyé de l'argent quand on faisait le chemin et tout ça. Quand on est arrivés ici ce n'était pas génial. On devait absolument travailler pour remplir l'argent des gens.

Il est intéressant de noter que la carrière professionnelle de M. Charles est en partie définie par ces choix initiaux; ouvrier non qualifié, il a des salaires minimaux, un emploi sensible aux périodes de récession de l'industrie, et il effectue un travail peu gratifiant. Cette situation peu satisfaisante l'amène aussi à changer d'emploi: "Ça ne me plaisait pas non, j'ai appris quelque chose, c'était une autre découverte, une autre chose c'est ce que je voulais, pour changer un petit peu entre-temps, mais ça donnait... Un peu

10. Mehmeti, 2013; Zittoun, 2006. 
c'était monotone. Et économiquement ce n'était pas aussi... C'était mal payé. C'était très monotone.» Cela l'amène aussi à avoir «une petite histoire avec mon patron justement aussi. J'étais obligé de quitter l'entreprise. [...] Et entre-temps j'ai eu le chômage, des cours et d'autres activités aussi.»

Le fait que son emploi ne soit pas très satisfaisant, ou ne fasse pas suffisamment sens pour lui, semble avoir amené M. Charles à développer de nouvelles sphères d'expérience. À la lumière des informations très modestes que nous avons sur son passé (réfugié politique, études en lettres), nous pouvons faire l'hypothèse que sa famille avait une éducation certaine, ce qui expliquerait aussi les engagements et l'envie d'apprendre de M. Charles:

Mais j'ai toujours eu envie de découvrir d'autres choses, de faire quelque chose de plus, je n'aime pas rester dans mon coin et rester fermé. Voilà! Je faisais la danse, je faisais le sport, après je faisais le foot, j'ai arrêté le foot, après j'ai commencé d'autres sports, j'ai commencé dans une salle de fitness, j'ai suivi quand même pendant des années. Après je me suis inscrit aussi dans une salle d'arts martiaux, j'ai fait sport d'arts martiaux, pis après j'ai été obligé d'arrêter. Après il n'y a pas eu le temps vraiment pour me mettre avec la famille, tout ça.

En effet, en parallèle, M. Charles retrouve une jeune femme, amie de sa sœur, qui vivait dans le même quartier que sa famille dans leur pays d'origine, et qui fréquentait la même école. Celle-ci est domiciliée dans un autre pays d'Europe, puis le rejoint et l'épouse en Suisse.

\subsubsection{ENTAMER UNE DÉMARCHE ADMINISTRATIVE:}

\section{LA DEMANDE DE NATURALISATION}

Après dix ans de séjour en Suisse et au bénéfice d'un permis C, M. Charles dépose fin 2003 une demande de naturalisation, justifiant ainsi sa demande:

Je suis arrivé en Suisse à l'âge de 17 ans avec mes parents. J'ai appris le français et aussi le métier que j'exerce depuis l'âge de 20 ans. Je me sens bien intégré dans le canton de Neuchâtel et je considère la Suisse comme mon pays. Je me suis marié ici et j'aimerais y vivre pour toujours en tant que citoyen à part entière 
partageant le destin de ce pays qui m'a accueilli pour le meilleur et le pire. La quasi-totalité de mes amis sont des Neuchâtelois et Suisses, je partage avec eux presque tout et c'est aussi ce sentiment de bien-être social qui me dicte l'envie de devenir un citoyen suisse. (Texte manuscrit, Formulaire de demande)

Ces arguments sont ceux qui sont fournis à l'administration, et ils ont évidemment pour but de convaincre le lecteur du bienfondé de la demande: M. Charles se sent "intégré», ce qui se manifeste par le fait qu'il travaille, parle le français et a des amis en Suisse. Toutefois, il est intéressant de souligner que M. Charles ajoute aussi cette phrase plus programmatique: «Je me suis marié ici et j'aimerais y vivre pour toujours en tant que citoyen à part entière partageant le destin de ce pays qui m'a accueilli pour le meilleur et le pire.» Pour signifier son lien au pays, M. Charles utilise un langage qui est proche de celui du mariage ("pour le meilleur et le pire») et implique un engagement à long terme ("vivre pour toujours comme citoyen à part entière»). On peut faire l'hypothèse, étant donné la mention du mariage en début de phrase, que M. Charles évoque la fondation d'une famille et que la citoyenneté «entière» implique donc le droit de participer pleinement à la vie politique et sociale, ce qui la distinguerait d'une citoyenneté "partielle». Dans ce sens, la demande de naturalisation manifeste aussi une certaine imagination de soi et de sa vie à venir.

\subsection{3 ÊTRE CONFRONTÉ AU TRAVAIL DE L'ADMINISTRATION}

La procédure suit son cours : l'État délègue au canton, qui délègue à la commune de domicile, qui charge la police de faire une enquête sur l'intégration de M. Charles (voir chapitre 5). L'officier de police en charge prépare un document résumant divers aspects de la vie institutionnelle de M. Charles (formation, état civil), et semble avoir consulté chacun des derniers employeurs de Charles; il indique par ailleurs que $\mathrm{M}$. Charles «s'exprime passablement bien en français » et qu'il "pratique le football au FC [régional] et diverses autres activités sportives" (rapport de police, novembre 2005, dossier adminstratif). À ce stade, il n'y a pas d'autre consultation de témoins.

Après plus de deux ans d'attente, $M$. Charles reçoit une lettre de l'Office fédéral des migrations lui indiquant que l'autorisation 
préalable ne lui est pas accordée: "Nous devons vous faire savoir que les autorités cantonales consultées ont émis un préavis négatif à l'endroit de votre requête. Elles font en particulier valoir qu'elles doutent de votre intégration dans notre pays" (courrier SEM à M. Charles, mai 2006).

M. Charles réagit immédiatement; il consulte l'administration communale, qui donne les raisons de ce refus. Ainsi, lors de l'entretien, dix ans après, $M$. Charles raconte cela de la manière suivante: "Alors, leur motivation c'était, ils avaient le doute, c'est ce qu'ils disaient, ils avaient le doute sur mon intégration. Et aussi, j'étais instable sur mon travail professionnel. J'étais instable» (M. Charles, juin 2014).

L'argument de l'instabilité professionnelle ne figure pas dans la lettre qu'a reçue M. Charles; il l'a appris des services, mais c'est celui qui le marque. De fait, les interruptions professionnelles de M. Charles paraissent liées à de nombreux facteurs, comme son parcours de formation, la situation du marché, la recherche d'un travail plus stimulant, etc. Elles auraient donc pu être interprétées comme un gage de l'aspiration professionnelle de M. Charles et sa capacité à créer des conditions de vie plus favorables. Au contraire, ce parcours justifie apparemment le "doute» de l'administration. Ce terme est alors repris par M. Charles et compris comme une mise en doute de sa personne; en effet, il semble interpréter le terme "d'instabilité», utilisé par l'administration pour qualifier l'intégration, comme désignant un état psychologique personnel: "j"étais instable». Nous revenons sur la place du "doute» dans l'évaluation du cas de M. Charles au chapitre 8 (8.2.3). D'un point de vue subjectif, le "doute» quant à sa stabilité professionnelle semble perçu comme un doute plus fondamental sur sa personne. À ce moment, persuadé de la légitimité de sa demande, M. Charles consulte le Service du Délégué aux étrangers et écrit une réponse à l'Office fédéral des migrations demandant le maintien de la procédure. La lettre est longue et structurée, et soutenue par des arguments nuancés. D'une part, elle explique de manière argumentée le fait qu'il ait connu une brève période de chômage, mettant ce fait en lien avec la situation socio-économique plus large, et montre son engagement professionnel. D'autre part, la lettre évoque tous les autres domaines dans lesquels «l'intégration» d'un candidat à la nationalité pourrait être attendue: vie associative, respect de la juridiction suisse, situation financière. M. Charles peut donc 
faire la preuve, par sa lettre informée, en bon français, qu'il est de fait «intégré»; il montre à l'appui diverses sphères d'expérience significatives pour lui. En outre, la lettre contient cet appel à reconsidérer sa situation avec cette formule étonnante: "Je pense sincèrement que les autorités cantonales et communales, qui nous ont donné des exemples formidables d'ouverture à l'égard de ses concitoyens issus des migrations, vont réexaminer le préavis en question" (lettre de M. Charles à l'Office fédéral des migrations, juin 2006). M. Charles fait en effet appel à «l'ouverture» des services à l'égard des autres "concitoyens issus de la migration": cette phrase, qui n'est pas sans flatter son destinataire, montre aussi la connaissance que Charles a de l'histoire du canton (voir chapitre 4) et des coutumes en vigueur, et, implicitement, indique encore une fois qu'il n'est pas moins qu'un autre apte à devenir un «citoyen à part entière». M. Charles se montre à ce moment proactif, informé et compétent, défendant un projet qui continue à faire sens. Au chapitre 7, nous revenons sur l'agentivité légale que manifeste ici M. Charles. À la suite de cela, une nouvelle enquête est demandée par l'Office fédéral des migrations à la commune.

La deuxième enquête est ouverte en 2006; deux témoins probablement suggérés par Charles sont consultés. Le dossier ne nous permet que de voir la réponse d'une employée du fitness qu'il fréquentait et qui le présente comme quelqu'un d' "agréable envers chacun, $[\ldots]$ correct, très poli» et qui "paye ses cotisations" (lettre de témoin au Service de la justice, septembre 2006). Après une pause de deux autres années, un rapport est effectué par un autre officier de police, en 2008. On y apprend que la situation professionnelle de $\mathrm{M}$. Charles a évolué et qu'il a un contrat à durée indéterminée. Il a aussi ouvert sa pizzeria, où il emploie du monde, grâce à des économies et à un emprunt. Selon le rapport, "l'intégration» est évaluée au fait que M. Charles a moins de temps pour son club de football, mais qu'il fait néanmoins du sport. Le rapport de police se conclut sur cette évaluation énigmatique: «M. Charles nous paraît être une personne équilibrée et bien établie sur sol neuchâtelois. Malgré qu’il ouvre la énième pizzeria/kebabs, il montre des prétentions concernant son avenir professionnel» (lettre de la police cantonale adressée au Service de la justice, août 2008). Toutefois, c'est là aussi qu'apparaît une indication de démêlés avec la police - où une plainte pénale est retirée en raison de l'absence de charge - et de poursuites (pour moins de 10000 fr.). 
Là encore, l'évaluation de l'intégration concerne essentiellement la sphère professionnelle et sportive de M. Charles. Il n'est pas fait mention de ses capacités linguistiques ni de son engagement politique en tant qu'élu communal, que nous savons par ailleurs exister. Le policier fait une évaluation presque psychologique, puisque $\mathrm{M}$. Charles est décrit comme une "personne équilibrée» - expression qui pourrait sembler répondre à "l'instabilité» antérieure. L'évaluation est également normative, mais ambivalente, faisant de $\mathrm{M}$. Charles une personne à la fois «bien établie», mais avec des "prétentions professionnelles». Néanmoins, le préavis est de nouveau négatif, invoquant «le non-paiement des contributions publiques de l'intéressé, les poursuites pendantes et le fait qu'il soit connu des services de police» (lettre du Conseil communal au Service de la justice, septembre 2008). Dans les faits, les poursuites ont été classées et le «non-paiement» consiste en un retard des impôts de l'année 2008 en cours. Ainsi, il est reproché à $\mathrm{M}$. Charles des faits qui sont bien postérieurs à sa demande de réexamen, en 2005, époque où il était solvable et connu pour payer régulièrement ses cotisations.

La décision peut paraître d'autant plus surprenante que M. Charles s'était efforcé de stabiliser sa situation professionnelle et financière lors de la demande de réexamen. Il engage alors un avocat, qui ne peut que confirmer cette situation, malgré la recherche d'arrangement auprès des impôts de M. Charles. L'affaire bouge peu et, sans nouvelles, $M$. Charles écrit à nouveau au Département de la justice en juin 2011. Cette fois, la lettre a un ton très différent de la première. Elle informe le département qu'il est divorcé et qu'il s'est remarié - ses sphères d'expérience familiales ont connu des transformations importantes -, qu'il a toujours une activité professionnelle stable à $100 \%$, qu'il exploite une pizzeria avec son épouse «en vue de développer cette activité qui nous l'espérons sera pourvoyeuse d'emploi». Il précise aussi que sa «situation financière est tout à fait saine», ne faisant l'objet d'aucune poursuite, et... :

[qu']au niveau des contributions publiques, je suis à jour, et j’ai obtenu un arrangement de paiement [...]; ce retard était dû à des problèmes comptables occasionnés par le début de notre activité de notre pizzeria et aussi par le problème du divorce de mon ex-épouse. (Lettre de M. Charles au Département de la justice, juin 2011) 
Enfin, M. Charles poursuit:

Je me fais un point d'honneur de m'intégrer le plus possible dans la vie sociale et professionnelle de notre région. Comme vous le savez, je suis membre de la Commission [communale]. [...] Je précise, en lien avec le rapport de naturalisation établi par la Police cantonale [...], que l'épisode lié à un accident de la circulation est définitivement réglé. [...] Comme indiqué, cette affaire est isolée, et je me fais un point d'honneur de respecter les règles de la circulation routière. (Lettre de M. Charles au Département de la justice, juin 2011)

M. Charles a changé de ton : il promet maintenant de se comporter en citoyen modèle, mettant un "point d'honneur" à s'intégrer "dans la vie sociale et professionnelle» et à respecter les règles de la circulation; il indique également avoir réglé sa situation financière. Ainsi, M. Charles attire l'attention des autorités sur certaines sphères d'expérience qui n'ont pas été mentionnées ni examinées auparavant: son engagement civique dans la commune, son esprit d'entreprise "pourvoyeur d'emploi", sa vie familiale. Il s'efforce aussi d'effacer les soupçons qui touchent aux aspects de sa vie qui ont été identifiés comme problématiques, à savoir ses impayés et sa conduite automobile. M. Charles reçoit une lettre huit mois plus tard lui disant que son dossier a été classé et qu'il peut, s'il le souhaite, recommencer la procédure (lettre du Département de la justice, de la sécurité et des finances à M. Charles, février 2012).

\subsubsection{LE SENS PERSONNEL DU REFUS}

Du point de vue administratif et légal, les raisons des deux préavis négatifs, qui courent sur dix ans, sont d'abord une «instabilité professionnelle» (dont le sens est transformé par le travail de l'écrit, voir chapitre 8), puis, lorsque celle-ci est stabilisée, des impôts en retard. Une affaire pénale sans suite est également, probablement injustement, invoquée. Évidemment, M. Charles est fâché et déçu, comme en témoigne l'entretien effectué avec notre équipe en 2014 . Il revient alors sur son envie d'obtenir la nationalité suisse, pour avoir...

...la meilleure vie, comme tout le monde! Comme le citoyen normal, je veux dire! Je ne vois pas... Je ne me différencie pas avec d'autres personnes en fait, voilà. C'est pour ça que peut-être ma 
réaction elle est un peu bête, mais je ne fais pas de différences, je me mets pas dans un statut étranger! C'est peut-être aussi pour ça, on me met déjà un statut étranger. [...] Je crois que moi je suis chez moi et pis je suis chez moi! J'ai tout appris ici, j'ai ma vie ici... (M. Charles, juin 2014)

Au regard de sa demande initiale, on peut proposer l'interprétation que, ayant vécu son adolescence et toute sa vie adulte en Suisse, de manière active et engagée, professionnellement, dans ses loisirs et sa vie civique, $M$. Charles a initialement souhaité une validation, ou une reconnaissance de ce qu'il perçoit comme un état de fait: il vit en Suisse et s'y sent chez lui. La demande initiale ainsi que la lettre d'objection de M. Charles au premier préavis négatif se lisent comme les énoncés d'une personne sûre de ses droits, et faisant confiance en des arguments raisonnables pour soutenir une demande légitime.

La durée et l'opacité de cette procédure amènent $\mathrm{M}$. Charles à adopter une autre posture; celle, dans sa dernière lettre, d'une soumission et d'une obéissance résignée. C'est aussi de cette manière qu'il en vient à comprendre la demande de l'administration; alors qu'il était quelqu'un qui s'est "battu» pour clarifier sa situation, il devrait donc apprendre à se taire: "J'arriverais, bien sûr, j'arriverais à fermer ma gueule, qui ne cherche rien, qui ne pose pas de questions, qui.... Quelqu'un qui n'essaie pas de chercher à apprendre, qui ne s'informe pas." Autrement dit, la négation répétée de sa demande amène $M$. Charles à une transformation identitaire: d'une personne active, il pourrait devenir passif et obéissant, et renoncer aux compétences dont il a fait montre jusque-là (ses capacités critiques, argumentatives). Sa lettre semble suggérer que cette transformation s'est déjà faite. Cette transformation identitaire le renvoie alors à une catégorie sociale dont il ne s'était jusque-là apparemment peu soucié, le fait qu'il est un «étranger» et qu'il n'a dès lors pas les mêmes droits que les autres:

Et bien sûr, nous on nous met, les autorités politiques, ils nous mettent dans une autre classe bien sûr; on est des immigrés, on est des étrangers! À leurs yeux. [...] Nous on doit se battre encore plus pour arriver au même niveau que le citoyen suisse bien sûr! On n'a pas les mêmes efforts à faire! On doit faire encore plus, toujours plus. 
Enfin, il faut noter qu'au terme de ces dix ans de procédure, et même encore au moment de l'entretien, M. Charles, pourtant bien informé, énonce que c'est la nationalité qui lui est refusée («ma demande de naturalisation a été refusée deux fois»); il semble ainsi n'avoir pas compris qu'il ne s'est jusque-là agi que de l'autorisation préalable, c'est-à-dire de l'autorisation fédérale (voir chapitres 5 et 7).

\subsubsection{LES DÉMARCHES DE NATURALISATION}

\section{DANS LA TRAJECTOIRE BIOGRAPHIQUE}

Après une enfance et un début d'adolescence soumis à la violence d'une crise politique et une rupture biographique importante, M. Charles a vécu la moitié de sa vie en Suisse. Il s'y est formé, y a fondé une famille, a progressé professionnellement, a ouvert un commerce. Il a été actif dans la vie associative et il joue un rôle politique. M. Charles apparaît comme actif, intéressé, engagé, critique et curieux. D'un point de vue externe, il fait un parfait citoyen : il connaît parfaitement les "us et coutumes» locaux. La procédure de naturalisation aurait, selon $\mathrm{M}$. Charles, dû simplement lui donner une pleine reconnaissance de son statut, en le faisant ainsi devenir un citoyen "à part entière " et en lui permettant de concrétiser une imagination de soi comme Suisse. Cette procédure l'accompagne pendant dix ans, et met certaines de ses sphères d'expérience à l'examen, à l'étalon de normes en partie implicites: une trajectoire professionnelle linéaire et sans "prétention" (dans les termes du rapport de police), une situation fiscale et juridique irréprochable, des activités associatives régulières. La procédure ignore d'autres sphères d'expérience de $\mathrm{M}$. Charles, que celui-ci essayera tout de même de mettre en avant, comme sa vie d'entrepreneur ou son engagement politique.

Toutefois, la temporalité du travail de l'administration et les aléas biographiques, ainsi que leur part d'imprévisibilité, créent des effets d'interaction parfois cumulatifs: la nécessité d'un travail fixe, condition exigée par la première évaluation, est ajoutée au projet de l'ouverture d'un restaurant; ces aléas professionnels, cumulés au divorce, créent une situation financière difficile; des arrangements sont trouvés pendant un certain temps, mais les délais d'examen du dossier le confrontent à de nouvelles difficultés. C'est ce décalage entre trajectoires biographique et administrative qui donne à des observatrices et observateurs comme nous l'impression d'un 
traitement problématique, et qui, pour la personne concernée, apparaît comme faisant obstacle au projet de naturalisation. M. Charles semble également percevoir que la procédure a eu pour effet paradoxal de l'éloigner davantage de la nationalité suisse que ce ne l'était lorsqu'il l'a initiée: «Plus tu passes le temps ici, plus tu es embêté avec la loi, pourquoi, parce qu'au lieu de "plus tu passes le temps, plus tu dois [ $\mathrm{t}$ '] intégrer", c'est "plus tu passes le temps, plus tu te désintègres"."

Le cas de M. Charles met en évidence les décalages qu'il peut y avoir entre la trajectoire administrative d'une demande de naturalisation et la trajectoire biographique d'une personne. Ce décalage se joue à un double niveau. D'abord, chacune de ces trajectoires a sa temporalité propre; ainsi, le temps long du travail de l'administration l'amène à des réponses qui entrent en décalage avec les développements d'une trajectoire de vie. D'autre part, chacune de ces trajectoires est en partie opaque à l'autre: les acteurs de part et d'autre ne voient pas toujours, et ne comprennent pas non plus très bien, ce qui se passe de l'autre côté; ils avancent, avec leurs moyens, pour ainsi dire à tâtons. En conséquence, entre ces deux trajectoires, des transactions prennent place, où se joue notamment un chassé-croisé autour de cette obscure notion d'intégration: la personne qui fait la demande essaye de manifester ce qu'elle imagine que l'administration identifiera comme indice d'une intégration réussie, tout en étant plus discrète sur d'autres aspects; l'administration va chercher des traces de conduites qu'elle peut interpréter comme des indices de manque d'intégration, tout en reconnaissant certaines conduites et en en ignorant d'autres. Ce double décalage est-il propre au cas de M. Charles ou le retrouve-t-on ailleurs? C'est ce que nous allons maintenant voir.

\subsection{LECTURE TRANSVERSALE DES CAS}

Dans la section qui suit, nous reprenons l'ensemble des cas que nous avons rassemblés, à savoir cinq cas de personnes engagées dans des demandes de naturalisation avec des dossiers complets, et cinq personnes seulement interviewées, dont quatre ayant fait des demandes de naturalisation et une demande de prolongation de permis. En suivant la structure adoptée pour la présentation $\mathrm{du}$ cas de M. Charles, nous brossons ainsi rapidement les types de trajectoires vécues par ces personnes et présentons ce qui les 
amène à entamer une procédure administrative (6.3.1), la manière dont elles formulent leur demande (6.3.2), leurs expériences face au travail de l'administration (6.3.3) et en particulier face aux refus qu'elles reçoivent. Nous nous arrêtons un instant sur les raisons invoquées pour les refus de l'administration, que nous examinons de manière plus systématique (6.3.4). Enfin, nous mettons en évidence la manière dont ces procédures prennent part aux trajectoires de vie des personnes concernées (6.3.5).

\subsubsection{AVANT LA DEMANDE}

La majorité des personnes que nous avons interviewées, engagées dans des procédures de naturalisation ou de renouvellement de permis, sont arrivées en Suisse pour fuir des conditions de vie difficiles, voire mettant en danger leur vie ou celle de leurs proches, dans leur pays. Les seuls cas qui font exception sont celui de M. Johan, qui est né en Suisse de parents européens (il s'agit donc d'une naturalisation suivant la procédure simplifiée pour les personnes de deuxième génération, voir chapitre 5), et de celui de M. Gilbert, arrivé d'Afrique au bénéfice d'un permis d'étudiant.

Toutes les autres personnes ont été victimes d'événements violents - coups, violences physiques, abus - ou ont vécu avec leurs familles dans des conditions d'angoisse - menaces d'arrestation ou de mort. Les trajectoires leur permettant d'arriver en Suisse sont elles aussi longues et complexes: des voies de passage d'un pays extra-européen via des points d'entrée en Europe, puis par différents centres d'accueil en Suisse, des traversées de l'Europe avec de faux papiers par des voies complexes et à la merci de passeurs souvent peu scrupuleux, etc. ${ }^{11}$ Les événements dramatiques qui ont poussé les personnes que nous avons interrogées à la fuite, ainsi que ces voyages souvent effectués à un âge encore jeune ou sans la famille, les ont affectées. Elles en parlent peu, parfois de manière décousue, et nous n'avons pas poussé les personnes à détailler ces récits, qui ont certainement été racontés (et évalués...) de nombreuses fois lors des procédures administratives. Toutefois, ces éléments sont importants à rappeler, car c'est avec ces passés et ces ruptures vécues que les personnes que nous avons rencontrées ont commencé leur vie en Suisse et ont été confrontées aux demandes de l'administration.

11. Moret, 2006; Womersley, Kloetzer et Gogikian Ratcliff, 2017. 


\subsubsection{ENTAMER UNE DÉMARCHE ADMINISTRATIVE}

Les confrontations des personnes avec les services administratifs semblent se produire dans deux cas de figure majeurs. D'une part, la personne est confrontée à l'administration lorsque son statut ou titre d'établissement est remis en question, soit parce que c'est un permis à renouveler régulièrement sous certaines conditions (comme un permis B), soit parce que les conditions de vie de la personne changent (par exemple, une séparation d'un époux, par qui le permis a été obtenu). Dans ces cas, les demandes sont traitées selon la loi sur les étrangers. D'autre part, c'est évidemment le cas lorsque les personnes engagent volontairement les démarches visant à obtenir la nationalité suisse. Comme pour le reste de cet ouvrage, nous nous centrons sur ces cas de demande de naturalisation, qui sont traitées selon la loi sur la nationalité, mais nous ferons appel à d'autres cas lorsque nous montrerons l'expérience qu'ont les personnes de leur confrontation à l'administration.

Nous avons eu accès aux formulaires de demande écrits par les personnes et leurs justifications relativement standardisées, mais qui laissent tout de même poindre des nuances, et à leurs explications orales. Associés à ces demandes de naturalisation, exprimées à l'écrit ou à l'oral, on peut trouver trois types d'arguments. Les premiers font écho à ce que nous pourrions appeler une imagination de soi; en lien avec une relecture du passé et/ou des projets d'avenir, la personne se sent suisse, chez elle. Le deuxième type d'argument renvoie aux droits et devoirs citoyens. Le troisième découle des deux autres, et est lié à une demande de reconnaissance.

Les personnes qui entament une démarche de naturalisation la justifient le plus souvent en expliquant qu'une part importante de leur vie s'est déroulée en Suisse, et que la Suisse est devenue leur chez-soi. C'est ainsi le cas de la première partie de la justification écrite de M. Kirin: "Je vis en Suisse depuis plus de vingt ans et je m'y sens bien intégré et bien assimilé. C'est aussi pour moi une motivation" (formulaire de demande de naturalisation de M. Kirin, juin 2013). De manière complémentaire, comme le faisait aussi $\mathrm{M}$. Charles, la demande peut être justifiée par un projet tourné vers l'avenir. C'est le cas aussi pour M. Johan: "Je suis né à Neuchâtel il y a trente-sept ans, mes amis et ma famille sont ici, je suis l'heureux père d'une petite fille de 3 ans et je voudrais la voir grandir en tant que citoyenne suisse» (formulaire de demande de naturalisation de M. Johan). Dans ce même ordre imaginaire, 
M. Kirin évoque son lien à la Suisse: "J'aime ce pays. [...] Je suis motivé par ce que je ressens moi-même. Vis-à-vis de la terre. Pas vis-à-vis des gens» (M. Kirin, juin 2013).

En second lieu, on peut lire dans ces réponses des formulations en termes de droits et de devoirs. C'est ce qu'énonce explicitement la demande de M. François: "Je suis en Suisse depuis dix ans. Je suis diminué dans ma santé, mais la Suisse m’a accueilli. Avec la situation de conflit [dans mon pays], je pense poursuivre ma vie ici. J'aimerais avoir des droits et des devoirs" (formulaire de demande de naturalisation de M. François). La justification écrite de M. Kirin se poursuit de la même manière: "Je me sens le devoir de servir ce pays qui m'a permis de vivre en sécurité» (formulaire de demande de naturalisation de M. Kirin). De même, M. Charles évoquait sa volonté de participer à la société comme un «citoyen à part entière».

Dans les entretiens, les choses sont plus nuancées. M. Kirin mentionne de manière beaucoup plus pragmatique les avantages qu'il escompte de la nationalité ou, au moins, la levée de limitations:

Vous savez, en tant que permis $\mathrm{C}$ aujourd'hui, si je vais, supposons que j'ai par exemple des contacts par exemple [aux États-Unis] [...] et puis tac, j'ai des gens avec qui je peux ouvrir une boutique. Je suis obligé, six mois après, d'aller à l'ambassade suisse pour demander un report, qu'on me prolonge. [...] Mais si j'ai le passeport suisse, je n'ai plus ce problème-là. Donc je peux aller le temps qu'il faudra, quelque part, installer quelque chose, démarrer même une entreprise ou quelque chose, mais sans savoir à être obligé de signaler encore six mois après, de venir après, donc [...] c'est toutes ces possibilités-là [...] finalement, ce passeport, il vaut la peine. (M. Kirin, juin 2013)

$M^{\text {me }}$ Elvire souhaite aussi obtenir une sécurité que le permis $C$ ne lui offre pas: "Parce que normalement avec un permis C, c'est pas sûr! Quand ils veulent qu'on quitte, on quitte! Ils disent voilà c'est fini, c'est fini!» (M ${ }^{\text {me }}$ Elvire, juin 2014). En effet, le permis $C$ a d'une part une durée limitée, qui rend difficile la projection dans le temps, et peut d'autre part être révoqué facilement et donc est perçu comme dépendant de décisions parfois arbitraires.

$\mathrm{M}^{\text {me }}$ Elvire double cela d'un projet clair d'aspiration sociale pour elle et ses enfants: 
Ça me plaît beaucoup parce que j'avais fait des bonnes choses que jamais j'aurais réussi à faire chez moi. [...] Comme chercher du travail. Avoir de l'argent. Une bonne éducation pour ma famille. Je suis heureuse que ma fille soit infirmière. [...] Qu'elle a trouvé ses études, comme ça elle peut avoir sa nationalité, ça veut dire qu'elle peut aller un petit peu en haut. Avoir le médecin et toutes ces choses comme ça parce que pour aller en haut il faut [avoir] un passeport. ( $\mathrm{M}^{\mathrm{me}}$ Elvire, juin 2014)

$\mathrm{M}^{\mathrm{me}}$ Line, à qui la nationalité a déjà été refusée deux fois, considère plutôt la nationalité suisse comme quelque chose à quoi elle aurait droit: "Mais, après un long terme, c'est vrai que je travaille ici, donc $j$ 'ai fait comme tout le monde qui payent leurs factures. C'est à dire j'ai grandi ici, j'ai fini mes études ici... Je pensais rester ici au moins pour que la Suisse me reconnaisse comme je suis: un citoyen suisse!» ( $\mathrm{M}^{\mathrm{me}}$ Line, mars 2014).

En troisième lieu, il est important de noter la demande de reconnaissance contenue dans ses formulations; on le lit chez $\mathrm{M}^{\mathrm{me}}$ Line ci-dessus, qui souhaite "que la Suisse me reconnaisse comme je suis; un citoyen suisse». M. Charles évoquait le sentiment de faire partie des citoyens de deuxième classe, qui doivent travailler plus dur et faire davantage la preuve de leurs engagements pour obtenir les mêmes droits que des citoyennes et citoyens suisses. Comme nous le verrons, c'est en grande partie en termes de reconnaissance ou de non-reconnaissance que les personnes interprètent le travail de l'administration.

Il est intéressant de mettre ces points en regard des justifications données par 28 personnes observées par Letizia Roellin ${ }^{12}$ lors de leurs entretiens de naturalisation dans le canton de Neuchâtel. Roellin identifie les arguments invoqués et propose une analyse argumentative de leur pertinence dans le cadre du travail d'évaluation de l'intégration des candidates et candidats par des commissions communales. Elle montre ainsi que les arguments de durée de résidence en Suisse, s'ils sont des préalables à la recevabilité de la demande et liés au sentiment d'être chez soi, ne sont pas considérés comme valides par les commissions. Il en est de même pour l'argument de se sentir suisse ou de vouloir offrir un avenir à sa famille. En revanche, certaines variantes de ces justifications en termes de 
droits et de devoirs sont admises, en particulier si elles manifestent la volonté de la personne de participer à la vie économique. Tous les autres arguments - jouir des mêmes droits et devoirs - ne sont pas validés et sont considérés comme "un cas fallacieux lié au fait de considérer la naturalisation comme un acte de convenance pour pouvoir disposer des mêmes bénéfices que les Suisses ${ }^{13}$. Par opposition, les arguments qui montrent par des exemples concrets que la personne est "offreuse» de sa force de travail, de son travail associatif ou de son paiement des impôts peuvent être acceptés ${ }^{14}$, ainsi que ceux qui font la preuve que la personne a des amis en Suisse ${ }^{15}$. Roellin conclut son travail en soulignant combien les arguments considérés comme des critères d'intégration lui paraissent éloignés de ce que dit explicitement la loi sur la nationalité, et en indiquant que les candidates et les candidats à la naturalisation ne semblent pas connaître les critères sur lesquels ils sont évalués ${ }^{16}$. Quoi qu'il en soit, si l'on suit ces critères, les personnes que nous avons rencontrées ne semblent pas avoir d'emblée su faire appel aux arguments nécessaires que met en évidence le travail de Roellin.

\subsubsection{LES PERSONNES CONFRONTÉES AU TRAVAIL \\ DE L'ADMINISTRATION}

De toutes les personnes que nous avons approchées, seul M. Johan a connu une procédure simplifiée, étant né et ayant grandi en Suisse de parents européens. Trois incidents sont toutefois à noter. D'abord, alors que sa demande initiale, collective, incluait sa compagne, née dans un pays européen, sa fille et lui, il a reçu un courrier lui demandant de faire deux demandes séparées, car le couple n'est pas marié. Pour des raisons financières, le couple décide de faire seulement la demande pour lui et leur fille. Ensuite, le casier judiciaire de M. Johan fait mention d'une «appropriation illégitime» datant d'une dizaine d'années. Ce point a été abordé lors de l'entretien:

Quand j'ai eu la, l'entretien avec les conseillers là, sur, ouais y a eu une, quand j'ai déménagé, j'ai confondu une cave, ce n'était pas la mienne. J'avais deux clés donc euh, je devais vider les lieux, et puis

16. Ibid., pp. 54-55. 
j’ai tout ramassé, parce que je devais vider les lieux je devais, oui, faire la remise des clés, et j'ai tout ramassé et puis après ben, les gens qui avaient cette cave ont porté plainte comme quoi ils ont été cambriolés ouais, j'ai pas fait exprès bon j’ai tout rendu après je ne voyais pas à qui ça appartenait, et puis c'est, c'est juste ce point-là où ils m'ont demandé qu'est-ce qui c'était passé. Et puis je leur ai expliqué et puis ça c'est, c'est bien passé. (M. Johan, juin 2015)

De fait, l'incident n'est pas retenu comme argument contre la demande. Ainsi, a posteriori, M. Johan se dit avoir été assez confiant en son bon droit malgré cet incident: "Je ne voyais pas pourquoi ils ne m'accepteraient pas. J'ai fait deux-trois, deux-trois bêtises, mais c'était rien de grave, c'est à l'époque quand j'étais jeune." Enfin, M. Johan rapporte le point suivant, qui s'est produit lors de l'entretien avec la commission de naturalisation:

J'ai passé l'entretien [...] j'ai dû comme donner euh, mon CFC, ce que j'ai fait, ma formation, mes attestations de travail, où je travaillais, et différents emplois que j'ai faits [...]. Et puis ils m'ont dit que c'était, pourquoi je ne votais pas parce que j'ai dit que j'ai voté peut-être une fois, il m'a dit, j'ai dit parce que voilà, ça m'intéressait pas trop de voter, et puis il m'a dit quand, si jamais ça passe, qu'on accepte votre candidat, votre naturalisation, là, faudra quand même vous lancer à voter parce que c'est important quand même, donc comme citoyen suisse de voter. Pourquoi pas oui. On va, on va voir. Et puis ce que j'ai fait la dernière fois c'est que, on a reçu, on a reçu des, du matériel de vote là, et puis j’ai voté là. (M. Johan, juin 2015)

M. Johan estime donc qu'il s'est fait pardonner un incident et il note l'invitation à voter à l'avenir qui lui est faite. Il semble que ces deux points ne sont pas considérés comme des indices de non-intégration, mais comme des erreurs de jeunesse. Il est intéressant de noter la manière dont les témoins décrivent $M$. Johan: "Je pense qu'être plus intégré que lui, ce n'est pas possible", "Comme [M. Johan] est né en Suisse, il n'a pas dû s'intégrer comme un étranger et est totalement intégré depuis sa naissance. C'est un Suisse avec des papiers [européens] " (rapports d'audition de témoins, dossier de M. Johan). D’une certaine manière, l'administration semble avoir fait une évaluation comparable, puisque 
son dossier évalue une «intégration consolidée»; le soutien de son réseau amical semble ici étayer cette lecture. Le traitement de la demande de M. Johan contraste fortement avec les autres demandes que nous avons examinées. Vraisemblablement plus typique des procédures courantes dans le canton, elle se déroule facilement en tant que procédure simplifiée pour des personnes de deuxième génération. La procédure considère l'intégration consolidée sur la base de la scolarité en Suisse et ne comprend pas d'entretien de naturalisation. En contraste, les autres cas qui nous ont été confiés sont tous, d'une manière ou d'une autre, des cas difficiles. Cette différence se traduit dans les pratiques par le fait que les petites erreurs de M. Johan ne sont pas interprétées comme des manques d'intégration (l'infraction, le fait qu'il ne vote pas). En contraste, les cas qui ne bénéficient pas des conditions facilitées sont traités avec davantage de suspicion; des démêlés avec la police pour un incident routier ou un impayé, comme dans le cas de M. Charles, sont interprétés comme des signes plus suspects, voire comme des indices de manque d'intégration (voir 6.2.3). À l'exception du cas de M. Johan, toutes les personnes dont nous avons analysé les dossiers ont vu leurs demandes initier de très longues procédures, allant de deux à neuf ans. Après avoir déposé leur dossier, toutes les autres personnes ont reçu de la part de l'administration des demandes de renseignements complémentaires. De nouveau, bien que ces cas ne représentent qu'une minorité de ceux qui sont traités par les services cantonaux, il est important de noter la manière dont ces durées et ces demandes de compléments sont interprétées par les personnes qui les vivent. Comme nous allons le voir, elles les considèrent parfois comme l'expression d'intentions mauvaises à leur égard, ou comme l'indice de l'incapacité des services.

D'abord, certaines personnes voient ainsi dans ces demandes de documents complémentaires ou dans ces délais des actions délibérées à leur encontre. M. Bruno les perçoit comme «toujours des prétextes! Des prétextes pour ne pas entrer en matière» (M. Bruno, mai 2014). M. Charles voit aussi le cumul d'obstacles comme résultant d'une intention maligne de l'administration à son égard: «Et chaque fois ça me fait ce coup-là, je réfléchis, ça m’intéresse, au fait que dans le service je crois qu'il y a des gens qui magouillent, qui trichent qui... Ou quelqu' un qui veut minimiser la demande je veux dire! J'ai pensé ça quoi!»(M. Charles, juin 2014). C’est aussi ce que pense M. Gilbert, qui y voit une intention délibérée 
de contrôle de l'immigration: "Non moi je pense que c'est l'administration qui bloque l'intégration des gens! [...] Dans le sens où on vous met dans une position inconfortable de séjour [...] qui fait que vous ne pouvez pas vous intégrer! C'est-à-dire c'est le permis à compte-gouttes, le permis temporaire tout ça...» (M. Gilbert, novembre 2014).

Ensuite, certains voient dans le travail de l'administration, perçu comme lent, l'expression d'une incompétence: "Et malgré ça, il $\mathrm{y}$ avait cette décision fédérale, on a eu ce problème. Et faut dire que ça veut dire qu'il y avait des gens vraiment incompétents sur le niveau cantonal ici. Ils cherchaient tout simplement des problèmes, ils n'aident pas» (M. Bruno, juin 2014). Globalement, cela donne l'impression à de nombreuses personnes que les actrices et acteurs de l'administration refusent d'entrer dans un vrai dialogue et utilisent de manière défensive la loi ou leur pouvoir, comme le formule M. Kirin : «On respecte la loi. La loi qui fait quelque chose qui soit contraignant humainement parlant, en le sachant, et que ces gens-là [de l'administration] se cachent derrière, quelque part, ben... je ne sais pas moi, ils trichent» (M. Kirin, juin 2015).

Dans tous les cas, les personnes que nous avons interrogées perçoivent l'administration comme quelque chose d'opaque; même si certaines sont demandeuses de contacts face à face, et les obtiennent parfois en fonction de leur agentivité légale (chapitre 7), plusieurs souffrent de ces longues procédures dépersonnalisées et œuvrant par lettres interposées. M. François, par exemple, reçoit un refus à sa demande de regroupement et demande à le discuter: "Parce que moi je suis allé à la réception demander pour le rendez-vous et parler [au chef]. Discuter. [...] La dame a dit non, elle a dit nous on demande pour le rendez-vous. Envoyez ou téléphonez... ça, c'est la lettre comme ça» (M. François, novembre 2014). La procédure est d'autant plus opaque que certaines informations ne semblent pas circuler entre les services: "J'avais besoin d'un certificat pour l'invitation de mon frère et ma mère. Maintenant je n'arrive pas à comprendre non plus; ma commune d'origine est au courant que je suis naturalisé, [la ville de $\mathrm{X}$ ] aussi, sauf le service d'immigration » (M. Armand, mai 2014).

Dans les faits, cela se traduit notamment par une compréhension difficile et parfois erronée de la procédure. Les personnes doivent interpréter comme elles le peuvent les réponses positives ou négatives qu'elles reçoivent. Ainsi, $\mathrm{M}^{\mathrm{me}}$ Elvire et $\mathrm{M}^{\mathrm{me}}$ Dominique 
semblent associer l'octroi d'un permis de séjour à une récompense, récompense obtenue, car elles ont beaucoup travaillé: «Et puis on a travaillé durement et puis on a obtenu le permis $\mathrm{B}$ » $\left(\mathrm{M}^{\mathrm{me}}\right.$ Dominique, juin 2014) ou, comme le dit $\mathrm{M}^{\mathrm{me}}$ Elvire au sujet de son mari, «il a obtenu le permis $\mathrm{C}$ parce qu'il a travaillé» $\left(\mathrm{M}^{\mathrm{me}}\right.$ Elvire, juin 2014). M. Charles, de son côté, n’a simplement pas compris que toute la procédure qui l'a occupé pendant neuf ans ne concernait que l'autorisation préalable; pour lui, "ma demande de naturalisation a été refusée deux fois».

Au regard de ce travail de l'administration, les personnes réagissent différemment - de manière fataliste ou, au contraire, plus engagée (chapitre 7). M. Charles considère longtemps chaque difficulté de la procédure ou chaque demande complémentaire comme un problème à régler: "Si moi j'ai posé mon dossier de naturalisation, j'essaie soit de trouver une solution, soit quelqu'un qui me prête l'argent. [...] Je trouve une solution!» (M. Charles, juin 2014). M. Kirin, au contraire, se sent davantage victime: "Quoi qu'ils fassent de moi, tout ça... C'est pas ma faute» (M. Kirin, juin 2015). M. François, quant à lui, se sent carrément impuissant face à des demandes qu'il ne pourra jamais honorer, comme lorsqu'il doit faire la preuve d'un salaire suffisant pour pouvoir obtenir des visas pour sa famille restée à l'étranger, alors qu'il est au bénéfice de l'assurance invalidité:

Économiser. Il a dit travailler. Parce que donner argent, appartement ça coûte cher, pour l'assurance maladie, pour ça alors ça coûte cher! [...] Alors moi ce n'est pas possible! Moi malade à la bouche, c'est pas possible... Travailler normal va trouver! Jamais trouvé! Travailler normal. (M. François, novembre 2014)

Comme nous le verrons plus bas, cette confrontation au travail de l'administration amène parfois à percevoir celle-ci comme tolérante et arrangeante, comme pour M. Johan, ou au contraire comme une instance hostile, suspicieuse, incompétente ou animée par une intention de nuire (tricher, se cacher), ce qui peut avoir des répercussions à long terme pour certaines des personnes par ailleurs marquées par des trajectoires complexes.

\subsubsection{DES RAISONS DE REFUS ET DE LEURS CONSÉQUENCES}

Les neuf personnes ayant les dossiers compliqués que nous avons analysés ont donc, à un moment ou à un autre, reçu une réponse 
négative à une demande, qu'il s'agisse d'une demande de naturalisation pour soi et/ou ses proches, ou d'un refus de prolongation de permis, ou de regroupement familial qui découle de la situation. Voici la liste des arguments reçus, en lien avec les demandes de naturalisation (sauf indication contraire).

\section{TABLEAU 6.1 RAISON DE REFUS}

\begin{tabular}{l|l}
\multirow{2}{*}{ Raison de refus } & Personnes, $\mathbf{M}^{\text {me }}$ ou $\mathbf{M}$. \\
\hline \multirow{2}{*}{ Pas de revenu financier stable } & Charles $\langle$ ? $>$ \\
\cline { 2 - 2 } & Line \\
\cline { 2 - 2 } & Kirin \\
\hline Contrats de travail à durée limitée & Bruno \\
\cline { 2 - 2 } Connu de la police & François (demande de visa pour \\
& que sa famille puisse lui rendre visite) \\
\hline Réussite d'examens universitaires & Charles \\
\hline & Charles \\
\hline Port du voile & Gilbert (demande de prolongation \\
\hline Demande familiale incorrecte & de permis B) \\
\hline Connaissance insuffisante de la langue & Line \\
\cline { 2 - 2 } & Johan \\
\cline { 2 - 2 } & Charles \\
\hline
\end{tabular}

Ce qui est frappant dans cette compilation, c'est la place des arguments financiers: trois des dossiers sont bloqués pour des raisons de retard dans le paiement des impôts. En lien avec cela, les personnes doivent être indépendantes financièrement, ce qui justifie deux des refus, mais aussi, par extension, un des cas où la personne n'a pas de contrat de travail à durée indéterminée. Ainsi, les arguments de refus iraient dans le même sens que la lecture proposée par Roellin: l'acceptation des personnes paraît prioritairement liée à l'insertion professionnelle et, partant, à leur capacité financière.

Trois des refus sont plus clairement justifiés par des arguments liés à l'intégration, au sens juridique: deux pour une maîtrise insuffisante de la langue, et une des personnes est considérée comme non intégrée parce qu' elle porte le voile. Toutefois, lorsque 
l'on regarde ces arguments de plus près et dans le contexte biographique des personnes, on peut se demander s'ils ne traduisent pas un manque de souplesse face aux spécificités des cas ou de proportionnalité. Ainsi, la difficulté de M. François à maîtriser la langue française est liée à un traumatisme crânien lors des événements violents qui ont causé son exil initial, et qui prétérite ses capacités d'apprentissage - fait qui sera mis en évidence, avec succès, lors du recours déposé par son avocat. $\mathrm{M}^{\mathrm{me}}$ Dominique, quant à elle, a appris le français en arrivant en Suisse, mais a plus tard déménagé en Suisse alémanique pour suivre son époux; c'est là qu'une première demande a été formulée, qui apparemment n'a pas tenu compte du fait que l'allemand, que $\mathrm{M}^{\mathrm{me}}$ Dominique ne maîtrise pas, est la seconde langue nationale qu'elle apprend ${ }^{17}$. De son côté, M. Bruno, sans qualifications en raison de sa trajectoire en Suisse (permis de réfugié, etc.), a travaillé toute sa vie, mais l'établissement qu'il a récemment ouvert a fait faillite. M. Bruno est maintenant sommé de trouver un emploi fixe, alors qu'il a plus de 50 ans, qu'il se relève de ses difficultés et que le marché du travail intègre difficilement des travailleurs de son âge, même qualifiés.

En fait, il semble que les trajectoires des personnes dans des situations difficiles entrent souvent dans des cercles vicieux, où les difficultés s'enchaînent et se génèrent mutuellement, comme nous l'avons vu dans le cas de $\mathrm{M}$. Charles, où les décalages entre trajectoires administrative et biographique rendent les exigences de solvabilité de plus en plus difficiles à satisfaire. Voici quelques exemples où c'est le statut administratif même des personnes qui semble rendre de plus en plus difficile la satisfaction des critères requis pour une naturalisation.

M. François est dans la situation difficile d'être en partie à l'assurance invalidité en raison des événements qui sont aussi à l'origine de sa présence en Suisse et de l'obtention de la nationalité (après l'intervention d'un avocat). Il travaille en atelier protégé et une assistante sociale l'aide pour ses démarches administratives. C'est en raison de son statut d'assisté, donc de sa non-autonomie financière, que l'administration lui refuse le droit de faire appel au regroupement familial, alors qu'il n'a pas vu sa femme depuis

17. D’un point de vue légal, il pourrait y avoir dérogation sur la question de la maitrise de la langue parlée au lieu du domicile s'il y a eu un déménagement d'une région linguistique suisse à une autre (voir ci-dessus, 4.3.1). 
son exil, vingt ans auparavant. Il reste pénalisé par son statut, qui l'empêche de revoir sa famille et de sortir d'une solitude dont il souffre.

M. Bruno est arrivé en Suisse d'un pays en guerre, avec sa fratrie, en transit pour l'Angleterre dans les années 1980. Âgé de moins de 30 ans à ce moment, il obtient par sa famille un emploi dans le corps diplomatique, puis après huit ans, la situation politique étant toujours tendue dans son pays d'origine, il dépose une demande d'asile en Suisse. Contrairement à ses attentes, le permis F qu'il obtient n'est pas facilement converti en permis plus stable et est renouvelé tous les ans pendant huit ans - période durant laquelle il travaille dans différents emplois non qualifiés. Il obtient ensuite un permis $\mathrm{B}$ et occupe à nouveau divers emplois. Plus récemment, il est devenu propriétaire d'un café-restaurant. Là, les choses se sont compliquées, d'une part parce qu'il a eu l'impression de souffrir de racisme de la part de la population et, d'autre part, en raison de difficultés financières, qui l'ont amené à faire faillite et qui ont eu des répercussions sur sa santé. À ce moment, $\mathrm{M}$. Bruno risque le non-renouvellement de son permis en raison de cette faillite qui l'a conduit à l'assistance. Il essaye d'expliquer son cas :

Le conseiller d'État, il m'a dit, il faut vous trouver un travail. Mais! Allez-y trouver un travail! Ah ah. Il faut dire que, à 20 ans vous pouvez trouver un travail. À 29 ans vous trouvez. Mais à 40, 45, 51 ans... Vous n'avez pas de formation, qui, l'État n'est pas... Vous avez... ça veut dire, c'est la faute de l'État! Quand moi j'avais 29 ans ici, j'avais toutes les... les... années devant moi pour faire une formation! Mais parce que $\mathrm{j}$ 'avais un permis $\mathrm{F}$ je n'avais pas le droit. Et pis maintenant ils reviennent, ils me disent "vous êtes mal intégré parce que vous n’avez pas de travail» donc... L'État, en général il vous avait mis dans cette situation-là, et pis par la suite il vous punit. [...] Je suis revenu au niveau zéro, à la première étape avec l'administration! (M. Bruno, juin 2014)

Dans cette perspective, le cercle vicieux inclut ici le type de permis que $\mathrm{M}$. Bruno a obtenu, les restrictions en matière de formation et de parcours professionnel qui en découlent, et la précarité financière que celles-ci ont entraînée; c'est cette précarité qui devient maintenant la marque d'un manque d'intégration et qui pourrait amener à un renvoi de M. Bruno. 
$\mathrm{M}^{\mathrm{me}}$ Elvire, quant à elle, est arrivée en Suisse par la voie de l'asile, jeune et sans formation. Elle a obtenu un permis B par mariage, puis a divorcé et entamé les démarches pour obtenir la nationalité suisse pour ses deux enfants et elle-même. Sans formation, elle cumule des emplois à temps partiel - des ménages chez des particuliers qui l'embauchent depuis sept ou huit ans et un travail de nettoyage en soirée. Même si elle peut ainsi faire vivre sa famille, elle est dans une situation précaire dès lors que deux événements viennent perturber l'équilibre financier qu'elle a trouvé en vue des démarches de naturalisation. D'une part, elle a cru comprendre que pour le maintien de son permis ou pour voir sa demande de naturalisation aboutir après son divorce, la famille doit loger dans un appartement avec une chambre par enfant; elle a donc loué un cinq pièces alors qu'elle était précédemment logée chez des amis qui lui prêtaient deux chambres (une pour elle, une pour les enfants). Sans entrée de salaire après un accident, elle a pu trouver ce contrat de location grâce à un propriétaire compréhensif et aux apports financiers de sa fille. À cela s'ajoute, d'autre part, qu'elle a des problèmes de santé qui l'obligent à se faire opérer; elle est immobilisée pour plusieurs mois. Bien que ses emplois soient déclarés, elle n'a pas d'assurance maladie ou accident, et ses employeurs rompent leurs engagements. Apparemment, le chômage refuse de la payer: "Ils m'ont pénalisée parce qu’ils disent que c'est ma faute. Que c'est ma faute! Que je sois malade. Maintenant, le chômage ils me paient pas» ( $\mathrm{M}^{\mathrm{me}}$ Elvire, juin 2014). Seul l'emploi de nettoyage de bureaux semble faire fonctionner l'assurance maladie et elle est payée à $80 \%$ de son salaire partiel. Pour pouvoir vivre et payer les frais de dossier, sa fille, qui travaille comme infirmière la journée, vient la remplacer dans un de ses emplois de nettoyage de bureau le soir pendant son congé. Nous ne connaissons pas la suite de la demande de $\mathrm{M}^{\mathrm{me}}$ Elvire.

Dans les cas de $\mathrm{M}^{\mathrm{me}}$ Elvire comme de M. Bruno, et d'autres encore, l'administration semble considérer qu'un statut professionnel précaire ou l'absence de moyens financiers sont des critères d'une intégration insuffisante, alors même que les personnes perçoivent leur situation comme résultant des contraintes même que l'administration fait peser sur elles et des efforts qu' elles fournissent pour tenter d'y satisfaire. C'est ainsi ce qui fait dire à certains que les démarches de naturalisation freinent l'intégration (M. Gilbert), si elles ne causent plus radicalement de la désintégration (M. Charles). 


\subsubsection{LES DÉMARCHES DE NATURALISATION}

\section{DANS LA TRAJECTOIRE BIOGRAPHIQUE}

Marquées par de nombreuses difficultés avant que soit entamée une demande de naturalisation, les trajectoires biographiques analysées se voient ainsi infléchies par des trajectoires administratives qui durent parfois longtemps et qui sont, elles aussi, perçues comme éprouvantes. Face aux démarches, certaines personnes nourrissent un sentiment de révolte, de colère ou d'injustice; d'autres sont profondément abattues. Nous montrons ici tour à tour que ces démarches peuvent amener les personnes à remettre en question leur idée de la Suisse ou de la loi, que leur durée peut être dommageable et, plus généralement, qu'elles génèrent de l'anxiété; enfin, nous montrons qu'elles peuvent radicalement remettre en question le sentiment de continuité des personnes et la manière dont elles peuvent penser leur trajectoire de vie.

Premièrement, comme nous l'avons vu plus haut, face aux difficultés, de nombreuses personnes en viennent à considérer la loi comme injuste, ou du moins son application par les personnes qui la mettent en pratique: «Mais personnellement je trouve que la loi ce n'est pas la même chose pour tout le monde» (M. Armand, mai 2014); "La loi, ce n'est pas la bible! Un! Deuxièmement, elle n'a pas été écrite par Dieu! Donc elle a été écrite par quelques êtres humains pleins de défauts" (M. Bruno, mai 2014); d'après M. Bruno, l'application de la loi "pousse les gens à la criminalité». M. Kirin pense que "C’est la façon qui est mauvaise. La façon de l'appliquer est mauvaise» (M. Kirin, juin 2015). Il en vient à évaluer la difficulté plus générale de vivre en Suisse: "La Suisse [...] c'est un pays dur hein, [...] même pour les Suisses. Moi je connais des jeunes Suisses qui n'arrivent pas à s'en sortir sur le paiement de leurs factures. Les impôts, tout ça. Ils sont toujours endettés» (M. Kirin, juin 2015). Dans tous les cas, quelles qu'aient été les attentes des personnes vis-à-vis de la Suisse, la plupart en font une lecture désabusée: la loi y est appliquée de manière injuste, les professionnelles et professionnels se cachent et se défilent et les amènent à sentir qu'elles ne sont pas les bienvenues.

Un deuxième élément important est celui de la durée des démarches. Nous l'avons vu avec M. Charles, ces démarches peuvent accompagner une vie pendant plusieurs années, parfois une décennie. Cela implique donc que les personnes acceptent, ou du moins se voient contraintes de vivre avec le fait que divers 
aspects de leur vie peuvent être mis à l'examen et devenir des arguments déterminants dans l'évaluation de leur demande. La structure de la procédure expose donc les choix de vie à ce regard de l'administration par ailleurs impersonnelle. $\mathrm{M}^{\mathrm{me}}$ Line exprime la souffrance psychologique que cette mise à l'examen suscite: elle mentionne dans ses courriers à l'administration sa "maladie psychologique» (voir aussi chapitre 7), vraisemblablement une dépression. Elle évoque ainsi lors de son entretien qu'elle a perçu le refus comme «blessant»: "Ils jouent sur mon temps, sur ma vie, je trouve que... Je ne vois pas... Je n'ai pas été tuer quelqu'un. [...] Je trouve que ça devient angoissant, ça devient épuisant» ( $\mathrm{M}^{\mathrm{me}}$ Line, mars 2014). On ne peut s'empêcher de faire l'hypothèse que cette situation d'examen permanent et imprévisible peut réactiver certaines expériences difficiles dans les pays d'origine ou vécues lors de la trajectoire migratoire de beaucoup des personnes concernées; en tout cas, elle fait planer un sentiment d'angoisse permanent.

Ainsi, un troisième élément qui se dégage de notre lecture est la solitude et l'anxiété dans laquelle ces démarches placent certaines des personnes. Discrète dans son expression, $\mathrm{M}^{\mathrm{me}}$ Dominique exprime à sa manière également une grande souffrance. Arrivée en Suisse par la voie de l'asile et bénéficiant, par le regroupement familial avec son mari, naturalisé, d'un permis B, elle n'a pas obtenu la nationalité suisse lorsqu'elle en a fait la demande avec sa famille dans un canton alémanique. Lorsqu'on lui demande si elle veut raconter quelque chose en particulier de son expérience, $\mathrm{M}^{\mathrm{me}}$ Dominique prend enfin la parole plus longuement:

Oui. Je raconte les difficultés parce qu'avec les enfants, de travailler ce n'était pas facile pour obtenir un permis! Comme je vous avais dit, j'ai fait deux travails. Alors j'avais des enfants, mon mari il travaille, [...] les enfants restaient à la cantine. Puis chercher seulement le soir. Moi je rentrais à $22 \mathrm{~h}$ parce que je travaillais, je finis le premier travail, je finis à $17 \mathrm{~h}$, l'hôtel. On travaille durement! On donne parfois 20 chambres, 25 chambres, j'ai travaillé à hôtel $\mathrm{A}$, j'ai travaillé à hôtel $\mathrm{B}$, hôtel $\mathrm{B}$, j'ai travaillé à l'hôtel $\mathrm{C}$, ce n'était pas facile avec moi, avec les enfants c'était vraiment difficile. Je ne voyais pas les enfants souvent et puis mon mari aussi, lui il travaillait de $9 \mathrm{~h}$ à $14 \mathrm{~h}$, il rentrait à $18 \mathrm{~h}$, et puis il retournait au travail! Il rentre vers $22 \mathrm{~h}$ à la maison. [...] C'était vraiment le temps difficile, difficile. ( $\mathrm{M}^{\mathrm{me}}$ Dominique, juin 2014) 
Les propos de $\mathrm{M}^{\mathrm{me}}$ Dominique laissent deviner une grande solitude, notamment liée au fait que, durant une longue période, elle n'a pas pu comprendre ses enfants parlant l'allemand, langue qu'elle ignore. Plus tard, vivant en Suisse romande, l'un de ses enfants est placé dans une institution spécialisée; elle-même, sans attestation de formation, est sans emploi; son mari s'absente pour une durée indéterminée; enfin, elle se trouve à l'aide sociale. $\mathrm{M}^{\mathrm{me}}$ Dominique a reçu par courrier des «menaces» de renvoi («ils me menacent par des lettres»). Elle dit ainsi : "Je ne peux pas rester à la maison comme ça... ça m’énerve beaucoup.» Cet "énervement" s'exprime somatiquement: "La pression là ça me donne, maintenant je suis obligée, j'ai la tension, la tension très haute. Très haute. [...] À cause des menaces là. [...] Je prends les médicaments qui sont là» ( $\mathrm{M}^{\mathrm{me}}$ Dominique, juin 2014). $\mathrm{M}^{\mathrm{me}}$ Dominique se retrouve donc dans une situation "énervante», bloquée à la maison, sans le soutien de son mari, dans une situation de précarité financière, avec une menace de renvoi vers un pays qui lui fait "peur", et avec très peu de moyens pour agir. $M$. François évoque aussi plus généralement la difficulté de sa vie et sa solitude - «Toujours la même, la vie c'est dur, dur, dur» (M. François, novembre 2014).

Un quatrième élément qui semble résulter de ces démarches est l'ombre qu'elles font planer sur la trajectoire de vie même des personnes: elles remettent en question leurs projets d'avenir, ou leur imposent de se relier à un passé problématique. $\mathrm{M}^{\mathrm{me}}$ Line et $\mathrm{M}$. Bruno perçoivent le refus de nationalité comme une non-reconnaissance de leur vie passée pour l'essentiel en Suisse. $M^{\text {me }}$ Line dit ainsi: "J'ai fait ma vie ici! Je n'ai jamais pensé retourner dans mon pays, parce que de toute façon tout le monde meurt dans la guerre, [...] donc je n'ai jamais mis les pieds. [...] Pourquoi? Parce que personne qui va me reconnaître» ( $\mathrm{M}^{\mathrm{me}}$ Line, mars 2014). M. Bruno dit: "Parce que vous savez, je n'ai pas d'autre passé [que mon passé en Suisse]. Moi j'allais voir la responsable ici des permis. Elle me dit: "Vous êtes un étranger!" Je dis: "Écoutez, moi je suis Suisse, même si je n’ai pas de papiers suisses, je suis Suisse! Trente ans après mon établissement en Suisse, moi je n'ai pas besoin que vous vous me dites que je suis Suisse. Ok? Pourquoi? Parce que j'estime que quelqu'un qui passe trente ans, n'importe où, dans n'importe quel pays, il a des droits là-bas"” (M. Bruno, mai 2014). D'une certaine manière, les deux personnes perçoivent le refus de l'administration comme une atteinte à leur intégrité, ou un déni de leur vie passée en Suisse et de leur sentiment d'appartenance. 
Sur un mode un peu plus interprétatif, ces différents éléments amènent à faire l'hypothèse que, pour les personnes que nous avons interrogées, le refus de l'administration est symboliquement perçu comme un déni de la légitimité d'exister en Suisse, si ce n'est plus simplement d'exister. Les personnes qui ont vécu une ou des ruptures liées à des expériences migratoires ont dû reconstruire une continuité. Leur passé en Suisse devient alors la base de leur nouvelle continuité ${ }^{18}$, et la possibilité de se référer à un passé est aussi la condition de pouvoir développer une orientation vers l'avenir ou d'imaginer un futur ${ }^{19}$. Cela les renvoie parfois a un ailleurs traumatique dont ils ont dû préalablement se défaire. Dès lors que l'imagination de possibles pour-soi est invalidée, c'est le sens même de sa vie qui est remis en question.

Une telle lecture permet ainsi d'expliquer l'intensité du choc perçu par M. Armand lorsqu'il est menacé de perdre sa nationalité après une aventure extraconjugale, alors qu'il a obtenu sa nationalité par naturalisation facilitée par le biais de son mariage avec une Suissesse. Il engage alors une procédure de cinq ans, vécue comme "un vrai cauchemar». Cette affaire très éprouvante rejaillit aussi sur sa vie professionnelle: "Non, mais c'était tellement tellement dur! J'avais un commerce ici et puis, est-ce que le lendemain, je ne vois pas mon avenir. [...] C'était un cauchemar. T'as un commerce, mais est-ce que je laisse tout tomber? Même plusieurs fois je suis allé chez un médecin là parce que j'étais vraiment déprimé. Malgré tout, c'est vrai que je n'ai pas laissé tomber mon travail» (M. Armand, mai 2014). Au terme de cette période, alors que M. Armand fait une demande de visa pour que sa famille puisse lui rendre visite, il apprend qu'il est "inconnu » du Service cantonal des migrations. Cela provoque son désarroi: "À ce moment-là, je payais mes impôts, je payais, j'avais un commerce, je votais! [...] Je ne comprends pas comment je suis inconnu, je paye mes impôts, je prolonge ma carte d'identité et en plus ça vient de chez vous, j'ai un commerce...» (M. Armand, mai 2014). Ainsi, malgré son ancrage dans les réseaux sociaux et professionnels de sa région, la reconnaissance officielle de l'existence de M. Armand est fragile et semble menacée par l'arbitraire de l'administration. De même, $\mathrm{M}$. Bruno parvient à un constat de dégât irrémédiable, l'expérience de se voir refuser la vie qu'il était en droit d'espérer: "En tout cas,

18. Erikson, 1959; Zittoun, 2012.

19. Zittoun et al., 2013; Zittoun et Gillespie, 2016. 
ça m'a fait la plus grande connerie de ma vie. Moi, j'avais beaucoup de potentiel» (M. Bruno, mai 2014).

Néanmoins, certaines personnes parviennent, malgré les difficultés, à maintenir des projets d'avenir. Ainsi, en dépit des événements traumatiques passés et de la difficile arrivée en Suisse, $M^{\text {me }}$ Elvire semble avoir pu vivre sa migration comme une nouvelle fondation et, plus sereine, développer une philosophie de vie personnelle tournée vers l'avenir ${ }^{20}$ :

Depuis que j'ai mis un pied dans l'Europe, je dis maintenant c'est une autre personne. Je vais devant et devant c'est devant. Mais s'il y a un mur, tu le lèves et tu passes. [...] S'il me tombe, je me lève, si je me casse un pied je me lève et je continue à marcher. Mais je vais arriver à la montagne. C'est ça qui est bien. On va arriver ensemble. [...] Doucement, quand on voit un jour on va être là-haut. On va être contentes et on va se souvenir de toutes les choses qu'on a vues. ( $\mathrm{M}^{\mathrm{me}}$ Elvire, juin 2014)

$\mathrm{M}^{\mathrm{me}}$ Elvire est la seule des personnes parmi les personnes rencontrées qui développe une lecture de son passé qui ne remet pas en question une imagination d'un futur possible, en faisant appel à une métaphore de voyage et des montagnes à surmonter. Ce qui rend cela possible n'est évidemment pas explicable dans la limite des données dont nous disposons. Toujours est-il que l'exemple montre aussi que, précisément, les trajectoires de vie peuvent être marquées par des difficultés - dont des trajectoires administratives -, mais aussi que, en fin de compte, elles peuvent ne pas s'y laisser enfermer.

\subsection{SYNTHÈSE: LA TRANSFORMATION DES TRAJECTOIRES DE VIE}

Ce chapitre avait pour but de suivre les procédures de naturalisation du point de vue des personnes étrangères en se centrant sur leur trajectoire de vie et en mettant en évidence le rôle que les procédures y jouent (la ligne du bas de notre figure 1, chapitre 1). Nous avons cherché à reconstituer qui étaient ces personnes et d'où elles venaient lors du début d'une démarche de naturalisation (ou de prolongation de permis), et à identifier comment elles donnaient sens aux interactions avec l'administration et aux réponses que 
celle-ci leur donne. Nous avons ainsi vu comment ces personnes se positionnent au terme des procédures auxquelles nous avons eu accès, et comment cela les amène à changer leur regard sur leur demande, sur le pays dans lequel elles vivent, et parfois sur leur vie en général. Il s'agit maintenant de revenir à deux de nos hypothèses exploratoires initiales (chapitre 1): la quatrième, qui proposait que les personnes formulent une demande de naturalisation (ou de prolongation de permis) en fonction d'une certaine imagination de leur trajectoire passée et à venir; et la cinquième, qui suggérait que, en fonction de cela, la trajectoire administrativo-légale des demandes de naturalisation était susceptible d'engager une reconfiguration des sphères d'expérience de la personne.

En ce qui concerne la première de ces hypothèses, nous avons en effet vu que la plupart des personnes que nous avons interrogées ont globalement, tant dans le cas de la demande formelle de naturalisation qu'au cours de l'entretien de recherche, manifesté le souhait de voir valider, ou reconnaître, un état de fait: l'expérience d'avoir vécu une partie importante de leur vie en Suisse, d'y avoir construit un chez-soi, et d'y avoir développé des projets d'avenir. Tout en étant attentives au fait que ces demandes ont aussi été formulées pour convaincre une audience (l'administration et peut-être aussi, parfois, notre équipe), il n'en reste pas moins qu'elles traduisent une imagination de soi, au futur, dans une vie qui se poursuit dans toutes ses ramifications: avoir une famille ou/et des enfants avec soi, pouvoir se former, travailler, développer des amitiés, s'engager dans des loisirs, voire des activités associatives et civiques. Cette imagination est également le plus souvent ancrée dans une certaine lecture du passé et du présent, dans laquelle la période de vie que la personne a déjà vécue en Suisse occupe une place prépondérante. Dans ce sens, pour les personnes, il semble que la demande de naturalisation ou de prolongation de permis soit une demande de reconnaissance d'un état de fait: elles déclarent se percevoir comme suffisamment intégrées pour y avoir droit et, ce faisant, elles demandent une validation publique et légale de ce sentiment d'intégration ou d'appartenance à une communauté. Cette validation amène aussi évidemment des avantages et, en particulier, permet d'éviter les désagréments, voire les stigmates, dus au fait de ne pas en bénéficier.

Cette impression d'évidence - ces personnes sont déjà intégrées, donc elles peuvent, voire doivent être naturalisées - est à l'origine de bien des surprises: comme nous l'avons vu, les réponses de 
l'administration sont rarement rapides, et rarement d'emblée positives. L'évidence est remise en question; s'il apparaît rapidement aux personnes que le point de vue de l'administration est différent du leur, il leur est cependant difficile de pouvoir cerner ce point de vue et les attentes spécifiques de l'administration. C'est là qu'émerge alors, dans l'espace des transactions entre personnes et administrations, ce qui nous est apparu comme l'opacité de cette dernière. Premièrement, il est souvent difficile pour les personnes de comprendre ce qui leur est factuellement demandé, ou les critères à l'origine des réponses qui leur sont données; deuxièmement, elles ont rarement accès à ce point de vue de l'administration, parce que les délais de réponse sont longs et qu'elles ont parfois l'impression qu'il est difficile de rencontrer ses représentantes et représentants en face à face. Troisièmement, nous l'avons vu, les procédures parfois très longues semblent elles-mêmes peu cohérentes du point de vue des personnes, puisque des exigences nouvelles semblent apparaître en cours de route. Ainsi, globalement, certaines des personnes ont l'impression que l'administration se cache; d'autres ont l'impression que celle-ci a des intentions mauvaises, quoiqu'indéfinissables, à leur égard. De ces transactions peut donc émerger un double décalage: un décalage informationnel lié au fait que les personnes concernées, du côté de l'administration comme de la demande, peinent à adopter la perspective des autres; et un décalage temporel, entre la vitesse et la rythmicité de la trajectoire de vie, et celles de la trajectoire administrativo-légale. Si celle-ci découle souvent de facteurs complexes et structurels, il n'empêche que la vie des personnes avance... à la vitesse des expériences de vie, et que des inadéquations entre les demandes des uns et les réponses des autres peuvent s'accroître avec le temps.

En ce qui concerne notre deuxième hypothèse, soit la question de savoir si le fait d'engager une procédure administrative peut entrầner une reconfiguration de la trajectoire de vie, la réponse suit dans certains cas le premier point, et dépend évidemment de la manière dont la demande est évaluée. D’abord, il semble que dans tous les cas de refus, les personnes candidates en sont rapidement arrivées à remettre en question leur image parfois idéalisée de la Suisse et de son fonctionnement administratif et légal. Ensuite, nombre d'entre elles ont ressenti des conséquences de ce refus dans différentes sphères d'expérience, d'un point de vue symbolique, mais aussi beaucoup plus concret. $\mathrm{Au}$ niveau symbolique, certaines personnes se sont alors perçues, comme dans l'image qui leur était renvoyée, comme 
«moins» intégrées qu'elles ne pensaient l'être. D'autres, et en particulier en fonction de leurs trajectoires passées lorsque celles-ci les ont exposées (elles et/ou leurs familles) à des violences physiques ou psychologiques, en viennent à internaliser ce qu'elles perçoivent comme des manifestations de rejet de l'administration à leur égard: leurs demandes deviennent "douteuses» et certaines personnes se sentent alors devenir elles-mêmes "douteuses". Sur un plan plus concret, le fait de devoir répondre à des critères inattendus posés par l'administration peut d'une part engager les personnes sur des variations de leurs trajectoires imprévues jusque-là: trouver un emploi permanent, prioriser le remboursement d'une dette plutôt qu'une nouvelle activité professionnelle, etc. D’autre part, les délais ou les réponses négatives de l'administration peuvent aussi empêcher les personnes de concrétiser certaines trajectoires imaginées: voyager, revoir sa famille, étudier, accéder à certains emplois est rendu impossible. Dans certains cas plus dramatiques, le cumul de ces aspects symboliques et plus matériels amène les gens à une situation de grand désarroi. Symétriquement, une demande qui aboutit positivement, si elle a pu mettre en doute temporairement l'un ou l'autre de ces aspects, permet à la personne de se voir "reconnue» et admise au sein d'une communauté, et de bénéficier des possibilités parfois nouvelles que cela permet. Ainsi, d'une manière ou d'une autre, sur un plan symbolique ou concret, qu'il s'agisse de l'image de soi en Suisse ou du rapport aux autres, de la lecture du passé ou de projets de vie, une procédure administrative participe à l'évolution d'une trajectoire de vie.

Se pose alors la question de savoir quelles compétences les personnes peuvent aussi déployer pour s'engager dans des transactions plus efficaces avec cette administration; c'est ce que nous verrons au chapitre 7 . Enfin, il nous faudra revenir sur l'importance des changements vécus par les personnes alors que leurs trajectoires de vie se déroulent en dialogue avec des trajectoires administrativolégales elles-mêmes en évolution (chapitre 8); nous reviendrons donc plus loin à notre première hypothèse, soit la question de savoir dans quelle mesure nous avons là affaire à une institution de transformation (partie IV). 


\section{AGIR AVEC LA LOI: \\ RÉCITS ET ACTIONS}

FLORA DI DONATO ET ÉLODIE GARROS ${ }^{1}$

I objectif de ce chapitre est d'analyser les trajectoires administrativo-légales de cinq personnes: $\mathrm{M}^{\mathrm{me}}$ Line, MM. Charles, - François, Kirin et Johan. Elles font toutes l'objet d'une procédure de naturalisation ordinaire - M. Johan ayant toutefois suivi une procédure de naturalisation simplifiée au sens du droit cantonal - et leurs cas relèvent de l'application de la loi sur la nationalité dans sa version de 1990 et de la loi sur le droit de cité neuchâtelois de 1955.

Nous nous centrons en particulier sur la manière dont ces personnes se positionnent vis-à-vis des lois et des institutions et nous répondons à cette question: comment deviennent-elles actrices légales au cours de la procédure? Ceci est en lien avec à notre sixième hypothèse exploratoire (1.4.3), selon laquelle les personnes candidates à la naturalisation ne sont pas simplement soumises aux aléas de la procédure administrative, mais peuvent au contraire agir face à la loi ou aux démarches administrativo-légales en faisant usage de différentes compétences et ressources afin d'obtenir la nationalité tout en sauvegardant leurs identité, valeurs et croyances.

Le chapitre se structure de la manière suivante: en évoquant des positions de sociologie du droit, nous proposons d'abord une définition des catégories de conscience juridique et d'agentivité légale pour décrire comment chaque personne articule son parcours légal et administratif afin d'obtenir la nationalité (7.1). Sur la

1. Ce chapitre et plus particulièrement les analyses présentées sont le fruit d'une collaboration interdisciplinaire, nourrie par la rencontre des approches théoriques des deux co-auteures de ce chapitre, Flora Di Donato et Élodie Garros. Toutefois, celles-ci précisent que certains paragraphes ou parties de ce chapitre ont été réalisés spécifiquement par Flora Di Donato, à savoir l'introduction ainsi que les parties 7.1, 7.2, 7.2.1, 7.2.2, 7.3.4 et 7.5. Les co-auteures remercient Vincenzo Ferrari, professeur émérite à l'Université de Milan et ancien directeur de l'Institut international de sociologie du droit (Оñati), pour la relecture critique du chapitre. 
base des approches narratives de l'étude du droit, nous explicitons ensuite la méthodologie de re-construction des histoires légales (7.2). Comme cas de départ, nous proposons celui de $\mathrm{M}^{\mathrm{me}}$ Line, qui illustre les obstacles d'un parcours administrativo-légal ainsi que les solutions et les stratégies mises en place pour les surmonter (7.3). Après avoir identifié des modalités d'agentivité typique de ce cas, nous examinons les autres cas (7.4). Enfin, nous présentons les conclusions de notre analyse (7.5).

\subsection{CONSCIENCE JURIDIQUE ET AGENTIVITÉ LÉGALE}

L'analyse du droit se fonde communément sur l'étude formelle des textes de loi, de la jurisprudence et de la doctrine juridique (1.2.1). Cette approche classique du droit ne s'intéresse pas aux relations entre droit, individus et contexte. Au contraire, notre investigation, en se focalisant sur l'impact du droit dans la vie quotidienne, étudie ce lien.

Toute personne n'est pas experte du droit. Elle peut néanmoins en faire l'expérience, développer ses propres représentations de la loi et du système juridique, en relation avec un environnement socioculturel spécifique ${ }^{2}$. Plusieurs travaux menés par des sociologues du droit, regroupés sous l'appellation «recherches sur la conscience du droit» (Legal Consciousness Studies), ont contribué - depuis des années - à cette perspective d'analyse qui met en valeur la participation de la personne à la construction du droit. Ces nouvelles orientations ont ainsi donné naissance au concept de conscience juridique (legal consciousness) ${ }^{3}$. Les sociologues du droit Ewick et Silbey invitent en particulier à considérer "la manière par laquelle le droit fait l'objet d'expérience et est compris par les citoyens ordinaires, dans la mesure où ils choisissent d'invoquer la loi, évitent de le faire ou lui résistent, est une part essentielle de la vie du droit» ${ }^{4}$. Elles distinguent trois manières dont les personnes non spécialistes se rapportent au droit.

- Face au droit (before the law). Le droit est défini par sa rationalité, son formalisme, son impartialité. Les personnes se situant face au droit revendiquent la légitimité des procédures légales, attestent leur confiance, revendiquent l'autonomie du droit.

2. Béal, Kalampalikis, Fieulaine et Haas, 2014; Clémence et Doise, 1995 ; Fieulaine, Kalampalikis, Haas et Béal, 2013.

3. Pour une définition complète du concept de legal consciousness, voir Ewick et Silbey, 1998; Silbey, 2008; Silbey, 2014.

4. Ewick et Silbey, 1992, cité et traduit par Pélisse, 2005, p. 8. 
- Avec le droit (with the law). Le droit correspond à un ensemble de compétences, de négociations, de stratégies. Il y a des règles, mais celles-ci peuvent être négociées selon les besoins. Le droit est défini par les auteurs comme une arène dans laquelle se déroulent des luttes stratégiques. Ce rapport laisse de la place à l'agentivité de la personne et à sa capacité à intervenir dans le système. Les personnes se situant avec le droit l'utilisent comme un outil pour accomplir leurs buts.

- Contre le droit (against the law). Les personnes présentent le droit comme étant arbitraire et se sentent impuissantes et/ou incapables de jouer avec ses règles. Elles sont contraintes de bricoler avec les ressources dont elles disposent pour lutter contre le droit: par exemple, ne pas répondre aux courriers, ne pas aller à certaines convocations ou encore s'exprimer avec colère.

Cette façon de conceptualiser le rapport au droit contribue à dépasser une vision purement instrumentale et formelle du droit. Toutefois, elle fournit peu d'informations sur la manière dont les non-experts passent de la représentation à l'action.

Nous proposons donc d'élargir cette perspective de départ en examinant non seulement comment la loi est vécue ou perçue par les personnes qui font une demande de naturalisation, mais surtout comment elle est mise en œuvre par ces personnes. Nous faisons l'hypothèse que l'agentivité légale de la personne candidate a un impact sur l'aboutissement de sa demande de naturalisation. Nous parlons dans ce cas de personnes légalement "actives " 5 .

La notion d'agentivité a été élaborée pour la première fois par les sociologues et les linguistes, dans les années 1970, afin de montrer comment les actions humaines peuvent avoir un impact sur les institutions. Ce concept a été développé ultérieurement par des chercheurs s'inscrivant dans l'école du pragmatisme afin de mettre en évidence la dimension de performativité du langage en contexte $^{6}$.

Dans le domaine de la psychologie, selon certains auteurs, comme Bruner, l'agentivité se fonde sur des compétences personnelles (cohérence, patience, sacrifice, intelligence) et sociales (capacité d'activer des expertes et experts, les amies et amis, la famille, les

5. Di Donato, 2020.

6. Pour une définition générale du concept d'agentivité, voir Ahearn, 2001, ou encore Eteläpelto, Vähäsantanen, Hökkä et Paloniemi, 2013. 
collègues dans le contexte professionnel; capacité de persuader et faire légitimer socialement ses propres choix) et sur la compréhension du système (règles formelles et informelles) ${ }^{7}$. Ces compétences qui sont propres à chaque personne sont socialement modelées, en ce sens qu'elles dépendent du type d'interactions que la personne elle-même établit avec l'environnement social. En adaptant cette notion au domaine juridique, nous définissons l'agentivité légale comme la capacité des individus à agir dans des cadres administratif et légal.

Ainsi, nous observons la manière dont les candidates et les candidats à la naturalisation - à partir de leurs compréhensions, attentes, expériences et positionnements vis-à-vis des lois et des institutions - développent progressivement des connaissances expertes du système légal et mobilisent leurs compétences pour agir en accord avec la loi et les institutions, construisent des stratégies d'action et négocient le sens de certaines notions (par exemple celle d'intégration) afin d'obtenir la nationalité. Nous verrons que si tous les parcours présentent des aspects communs inhérents aux impératifs de la démarche administrative, par exemple remplir le formulaire de demande de naturalisation, chacun d'entre eux est modelé d'une façon originale et unique ${ }^{8}$. Le rapport à la procédure est conditionné par le parcours personnel, légal et social de chaque personne, ce qui influence aussi les manières de se manifester: par des courriers manuscrits ou dactylographiés pour des requêtes spécifiques auprès des administrations visant à obtenir des explications ou encore par des demandes d'aide, que ce soit à des avocates ou avocats, des associations culturelles, des travailleuses et travailleurs sociaux ou encore des amies ou amis.

\subsection{CONSTRUIRE ET ANALYSER LES HISTOIRES LÉGALES}

Pour rendre compte des dimensions multiples que revêt une trajectoire de naturalisation et analyser la manière dont les personnes concernées réagissent vis-à-vis des pratiques administratives et

7. Voir Bruner, 1997b, qui a établi une liste de ce qu'il a appelé les self indicators. Pour une contextualisation plus approfondie de cette notion dans le domaine légal, voir Di Donato, 2014 et 2020.

8. Voir Zittoun, 2012, pour une perspective socioculturelle des trajectoires de vie mettant en évidence la capacité du sujet à se positionner comme un individu unique et, plus haut, chapitre 6. Voir également les travaux de Zittoun et Gillespie, 2016, pour penser les liens entre agentivité et imagination. 
légales - en lien avec notre deuxième hypothèse exploratoire (1.4.1) -, nous combinons différentes analyses: celles des récits des personnes candidates, celles des entretiens menés avec les responsables des institutions concernées, et enfin celles des dossiers de naturalisation produits par le Service de la justice, et parfois par les personnes candidates elles-mêmes. Les histoires sont reconstruites à partir du point de vue de celles-ci, selon l'approche socioclinique de l'étude du droit que nous avons évoqué au chapitre 1 (1.2.2).

Cette approche a une double finalité: d'une part, rendre visible le lien entre la construction du droit et le contexte historique, culturel et social au sein duquel le cas trouve son origine et, d'autre part, mettre en exergue les mécanismes narratifs qui sont à la base de la modélisation du droit. En fait, comme suggéré par Amsterdam et Bruner, ou encore par White, raconter une histoire ne signifie pas reproduire la réalité, mais ordonner les événements, que ce soit dans la vie quotidienne ou dans un contexte juridique? Cet ordre n'existe pas dans la nature, il est créé selon le point de vue de la narratrice ou du narrateur, en prenant en considération les exigences de l'auditoire et les contraintes institutionnelles ${ }^{10}$. Dans le cadre d'une procédure légale ou administrative, les faits ne sont pas donnés in abstracto, mais fabriqués à partir d'actions et selon les rôles endossés par les protagonistes du cas ${ }^{11}$. Chaque trame narrative propose une théorie, une version du déroulement des événements ${ }^{12}$. Le plus souvent, il s'agit d'histoires conflictuelles dans lesquelles les objectifs de deux ou plusieurs acteurs sont en opposition: par exemple, ceux de la candidate ou du candidat qui souhaite obtenir la nationalité et ceux des actrices et acteurs administratifs qui sont en charge de veiller à ce que la demande soit conforme aux lois et aux pratiques.

Nous définissons ces actrices et ces acteurs comme des clientes et des clients. Si un tel terme peut être considéré comme péjoratif, évoquant une logique de consommation de l'administration publique dont la fonction serait plutôt d'offrir des services et non des biens de consommation ${ }^{13}$, elle a une signification positive dans le cadre des approches cliniques que nous privilégions dans notre analyse. À partir des années 1980, une littérature assez vaste est

9. Amsterdam et Bruner, 2000; White, 1981.

10. Bruner, 1991.

11. Di Donato, 2008a; Di Donato, 2020.

12. Brooks, 1992; Bruner, 2003.

13. Giauque et Emery, 2016; Linde, 2008. 
dédiée au rôle de la cliente ou du client dans le cadre des procédures légales et judiciaires, afin de souligner son émancipation et son autonomie par rapport aux professionnels et professionnelles du droit, les juges et plus particulièrement les avocates ou avocats, en marge des relations marquées par une asymétrie de pouvoir et par la technique juridique ${ }^{14}$. Dans ce cadre, des recherches consacrées à l'analyse des interactions des clientes ou clients avec leurs avocates et avocats ${ }^{15}$, ou avec les juges ${ }^{16}$, montrent sous quelles conditions personnelles, sociales ou professionnelles, clientes et clients sont capables de prendre des initiatives pour résoudre leur cas ou encore de coopérer avec des avocates et avocats dans la reconstruction des faits à partir de la spécificité de leur histoire ${ }^{17}$. L'idée sous-jacente est que ces personnes puissent agir comme des alliées ${ }^{18}$. Ainsi, l'un de nos objectifs, dans l'espace limité de cette recherche, est d'attirer l'attention sur le rôle actif que les personnes candidates à la naturalisation, définies comme clientes, peuvent avoir dans leur parcours de naturalisation.

\subsubsection{LES RÉCITS}

Afin de faire émerger les différents points de vue qui donnent vie à une histoire, conformément au cadre théorique proposé en introduction de cet ouvrage (1.2.2), au moins deux perspectives sont prises en compte ${ }^{19}$ : la perspective, parfois multiple, des employées et employés des institutions et la perspective de la cliente ou du client. L'objectif est de comprendre comment les récits des divers actrices et acteurs impliqués interagissent, entrent en conflit, s'éloignent et se rapprochent. Dans ce but, nous proposons dans ce chapitre une reconstruction des parcours de demande de naturalisation de cinq personnes, les transformant ainsi en cinq cas, ou histoires légales. Pour cela, afin de construire les cas en adoptant le plus possible un regard holistique qui rend possible une confrontation entre l'histoire racontée par la personne et celle qui est mise en forme par l'administration, nous avons combiné deux outils, l'analyse de récits ou d'entretiens et l'analyse de documents ${ }^{20}$.

14. Voir Haavisto, 2001. Pour une identification de différents types d'initiatives du client, voir Di Donato, 2008a, 2008b, 2011 et 2020.

15. Ellmann, Dinerstein, Gunning, Kruse et Shalleck, 2009.

16. O’Barr et Conley, 1988.

17. Di Donato, 2008a et 2011.

18. Pour des approches cliniques du droit, voir Alfieri, 1990-1991, 2016 ; López, 2017; White, 1997.

19. Daiute, 2014.

20. Pour cette combinaison méthodologique, voir Di Donato, 2008a, 2012a et 2020. 
Notre analyse utilise comme point de départ le récit de la candidate ou du candidat recueilli lors de l'entretien de recherche, ainsi que nos notes rédigées à la suite de l'entretien (voir chapitre 2). Ces personnes ont raconté leur parcours pour arriver en Suisse, les démarches faites pour obtenir un permis de séjour et demander la naturalisation, les obstacles rencontrés. Elles ont également partagé leurs représentations des lois et des procédures. Ainsi, lors de l'analyse, nous nous sommes fondées sur le sens donné à leur expérience passée et présente ${ }^{21}$. Bien que l'entretien soit le point de départ pour repérer le fil rouge de l'histoire et construire une trame narrative, ce sont les documents officiels qui nous ont guidées dans la reconstruction chronologique des événements. Ainsi, nous avons été attentives au contenu du récit ainsi qu'à sa forme, notamment à travers l'examen du vocabulaire employé et de la place prise par les émotions dans le discours, tant à l'oral qu'à l'écrit.

Une analyse qualitative et compréhensive de ces récits ${ }^{22}$, nous menant progressivement à la construction de cas, nécessite la prise en compte des voix d'autres actrices et acteurs de la procédure de naturalisation (que ce soient les professionnelles ou professionnels du secteur naturalisation du Service de la justice ou du secteur naturalisation du Service de la cohésion multiculturelle), les avocates et avocats mandatés par les candidates et candidats, en encore les conjointes ou conjoints. Dès lors, nous avons également pris en compte dans nos analyses des entretiens, enregistrés ou non, avec les professionnelles et professionnels de la naturalisation, mais aussi des observations réalisées au sein du Service de la justice et du Service de la cohésion multiculturelle.

\subsubsection{LES DOSSIERS}

L'analyse de documents a concerné le dossier administratif et le dossier personnel qui portent sur la demande de naturalisation, les enquêtes menées par la police ou le Service de la cohésion multiculturelle, les échanges avec et entre les différents services administratifs; les interventions éventuelles des avocates ou avocats et les décisions judiciaires (voir le chapitre 2). Cette analyse de documents correspond à plusieurs exigences. Elle permet de 
contextualiser ${ }^{23}$ les faits qui sont racontés ${ }^{24}$, d'objectiver la manière dont la réalité des histoires prend forme dans les documents, dans les traces écrites ${ }^{25}$. Enfin, elle permet d'expliciter les pratiques des institutions et les représentations qui leur sont associées ${ }^{26}$. L'analyse de ces documents s'est faite en plusieurs étapes. Le contenu des dossiers a été entièrement scanné, puis les documents ont été classés dans l'ordre chronologique. Ce premier travail a permis, au-delà de l'organisation, de prendre connaissance de manière précise des différents documents, et en particulier de ceux qui ont plus d'importance pour l'administration, comme l'enquête sur l'intégration. Cette étape a aussi été nécessaire pour comprendre, à partir des traces écrites, objets fabriqués par les différentes actrices et acteurs de la procédure de naturalisation, le déroulement de cette dernière. Les dossiers ont ensuite été lus, interprétés comme un ensemble, c'est-à-dire un tout constitué de différentes pièces retraçant le parcours d'une demande de naturalisation. Nous évoquons volontiers un travail d'interprétation, puisque nous agissons sur le matériel analysé.

Ainsi, l'histoire légale construite à partir des documents juridiques et des différents récits et entretiens autour du cas est le résultat d'un discours polyphonique autour de la démarche de naturalisation. Nous avons construit, en collaboration avec chaque candidate ou candidat, une histoire de leur naturalisation. La construction et l'analyse de ces histoires légales rendent compte à la fois des différentes représentations et du sens attribué aux événements qui jalonnent la procédure, des façons dont ils agissent ou réagissent, mais aussi des contraintes du cadre au sein duquel leurs demandes prennent forme.

\subsection{LES CAS DE MME LINE ET DE MM. CHARLES, \\ KIRIN, FRANÇOIS ET JOHAN}

C'est selon la méthode d'analyse décrite ci-dessus que nous présentons dans cette partie les cas de $\mathrm{M}^{\mathrm{me}}$ Line, MM. Charles, Kirin, François et Johan, ceci dans le but de retracer le lien entre la conscience juridique, l'expérience du droit et l'agentivité légale

23. Gutwirth, 2013.

24. Bonilla, 2015.

25. Ferraris, 2001, et Latour, 1995.

26. Sherwin, 2012-2013. 
développée par ces protagonistes. Afin de mettre en évidence l'articulation de ces liens et leur évolution, nous faisons le choix de ne pas présenter la totalité de leur histoire, mais de nous focaliser spécifiquement sur les étapes que nous avons identifiées comme clés dans la procédure de naturalisation. Nous verrons que les candidates et candidats oscillent entre plusieurs représentations et positions dans le cadre de leur démarche administrativo-légale. Leur expérience progressive du droit et de la démarche administrativo-légale tend à modifier leur posture au cours de la procédure. Le fil rouge qui relie ces histoires est la complexité des parcours de ces candidates et candidats à la naturalisation, que nous qualifions d'atypiques. Cette complexité se manifeste principalement à deux niveaux: au niveau de l'interprétation et de la vérification des critères d'intégration par les professionnels appliquant la procédure de naturalisation; et au niveau des difficultés que rencontrent les personnes candidates pour se conformer aux exigences d'intégration et répondre aux attentes explicites et implicites des administrations.

\subsubsection{SE POSITIONNER «AVEC LE DROIT " :}

\section{LE CAS EXEMPLAIRE DE MME LINE}

Le cas de $\mathrm{M}^{\mathrm{me}}$ Line $^{27}$ constitue un cas exemplaire (voir 6.2), au sens qu'on y trouve la plupart des éléments qui nous permettent de définir ce qu'est l'agentivité légale. Certains de ces éléments, sous une forme plus simplifiée ou avec des variantes, apparaissent dans les autres cas.

$\mathrm{M}^{\mathrm{me}}$ Line a déposé deux demandes. La première fois, en 1998, la Commission communale des naturalisations exprime un préavis négatif, avec la motivation d'un manque d'intégration et d'assimilation aux us et coutumes suisses (courrier de la Commission des naturalisations et des agrégations, septembre 1998). La deuxième fois, en 2006, le refus de naturalisation est motivé par le Conseil d'État neuchâtelois en raison du «retard dans le paiement des contributions publiques» (extrait du courrier du Conseil d'État, décembre 2009). À la suite de ce deuxième refus, $\mathrm{M}^{\mathrm{me}}$ Line s'engage dans un échange avec les administrations qui va durer plusieurs années afin d'obtenir la citoyenneté.

L'histoire de $\mathrm{M}^{\mathrm{me}}$ Line a la particularité d'être racontée et documentée, dans un premier temps, par son mari, M. Paul, lors d'un

27. Pour des reconstructions sous des angles différents du cas de $\mathrm{M}^{\mathrm{me}}$ Line, voir Di Donato 2015a, 2016b et 2020 . 
entretien réalisé en juin 2013. Le récit de M. Paul se situe à l'intersection de plusieurs histoires: celle de $\mathrm{M}^{\mathrm{me}}$ Line, celle de la famille de $\mathrm{M}^{\mathrm{me}}$ Line et la sienne. Il se fait également le porte-parole des personnes étrangères qui vivent une situation similaire en Suisse:

Chercheuse: Donc, est-ce que ça vous intéresse de raconter votre histoire?

M. Paul: Mais non seulement la mienne, mais en général ce que j’ai vécu et que j'ai vu parce que j'ai aidé, j'ai essayé d'accompagner et puis jai vu ce qui se passe au total ${ }^{28}$. [...]. Ça fait vingt-sept ans et demi que je suis là en Suisse et ma femme depuis 1979 elle est en Suisse, elle avait l'âge de 10 ans quand elle est venue avec les parents. Donc il y a l'histoire de ma femme, l'histoire de moi, et puis de sa famille, et après des autres étrangers qui justement vivent ici et donc j'ai beaucoup d'expérience si ça peut vous aider. ${ }^{29}$ (M. Paul, juin 2013)

Il se positionne en tant quacteur (et client) compétent, et légitime son intervention dans la procédure de naturalisation de sa femme. En préambule de son récit, il donne son point de vue sur la loi suisse quant à la naturalisation, en la présentant comme discriminatoire, en raison de la distinction entre la naturalisation facilitée et la naturalisation ordinaire:

M. Paul: [...] Les lois en général, quand on dit les lois, c'est égalité pour tout le monde, ça doit être égal, que ce soit un Suisse ou un étranger, ça doit être égal, ok? Maintenant, si on regarde la loi [...] de naturalisation, il y a déjà au départ des étapes, des conditions: première, c'est naturalisation simple et, deuxième, c'est naturalisation facilitée. Là, il y a déjà une contradiction.

Chercheuse: Oui, pourquoi?

M. Paul: Parce que, quand on dit la loi, c'est égal, égalité pour tout le monde, il n'y a plus d'égalité. On a fait déjà deux sections: on a fait facilitée pour les gens qui sont mariés avec un Suisse ou Suissesse [...] pour ceux qui sont mariés ou qui vivent ici et qui ont un partenaire suisse.

28. La mise en italique a pour but de mettre en évidence certains passages du récit.

29. Dans la suite du chapitre, nous soulignons en italique les passages des extraits qui illustrent nos analyses et qui sont commentés. 
À l'occasion d'un deuxième entretien - en présence de M. Paul et de $\mathrm{M}^{\mathrm{me}}$ Line -, le premier témoigne de son expérience, qu'il qualifie de juridique, et de son rapport au droit, en particulier en ce qui concerne les recours en matière d'asile:

M. Paul: J'ai étudié beaucoup de procédures. [...] J'ai beaucoup d'expérience juridique parce que je traduisais des trucs pour les gens, je faisais les recours pour les requérants d'asile.

Chercheuse 2: Ici en Suisse?

M. Paul: Ici en Suisse. Donc jétais attaché beaucoup avec le juridique. Donc c'est comme ça. (M. Paul, mars 2014)

Dès le début, M. Paul doute tant de la rationalité que de l'impartialité du droit. Il cherche à comprendre son fonctionnement et à jouer avec ses règles. Dans ce sens, il est avec le droit et s'engage, avec sa femme, dans un jeu d'actions et de réactions vis-à-vis des autorités afin de contester des décisions qui leur semblent incorrectes, voire injustes, pendant la procédure de naturalisation.

Nous allons reconstruire l'histoire de $\mathrm{M}^{\mathrm{me}}$ Line et $\mathrm{M}$. Paul autour du double refus de naturalisation et des actions mises en place par ces deux protagonistes afin d'obtenir la nationalité.

$\mathrm{M}^{\mathrm{me}}$ Line demande la naturalisation pour elle et ses enfants en 1998. À cette date, la procédure dépend de la loi sur la nationalité de 1990. Le préavis de la Commission communale des naturalisations et des agrégations est négatif pour des raisons liées aux us et coutumes. De plus, la commission estime que les motivations de l'intéressée quant à la naturalisation sont mal définies et que ses enfants ne sont pas intégrés à l'école:

Madame Line s'est présentée devant la commission dans une tenue traditionnelle. Elle admet n'apprécier que ce mode d'habillement. Elle a adopté, par son mariage, la religion musulmane. [...]

Les motivations de Madame sont véritablement mal définies. [...] Les enfants sont actuellement âgés de 4 et 5 ans. Ils ne sont pas, à ce jour, intégrés dans le cycle scolaire. Cette étape permettra certainement de mieux évaluer la capacité de la famille à s'intégrer. [...].

$\mathrm{Vu}$ les faits décrits ci-dessus, la Commission de naturalisation et d'agrégation constate que la requérante ne donne pas l'impression d'être intégrée ni d'avoir assimilé nos us et coutumes et décide, à 
l'unanimité, de donner un préavis négatif à sa demande. Elle souhaite qu'une nouvelle demande soit représentée lorsque Monsieur sera également en droit de le faire. D'ici là, le temps aura permis à la famille, au travers de l'intégration des enfants à l'école primaire, de démontrer sa capacité d'intégration. (Extraits du courrier de la Commission des naturalisations et des agrégations à l'autorité communale, septembre 1998)

Le préavis négatif est donc motivé par le manque d'intégration et d'assimilation de $\mathrm{M}^{\mathrm{me}}$ Line aux us et coutumes suisses et par le manque d'intégration scolaire de ses enfants; la situation du mari est aussi présentée comme problématique, car il n'a pas le nombre d'années de résidence requis.

L'analyse de ce courrier démontre une interprétation restrictive de l'art. 14 de la loi sur la nationalité de 1990 (let. a, b) de la part de la commission communale chargée d'examiner le cas de $\mathrm{M}^{\mathrm{me}}$ Line. Une telle interprétation semble contraster avec l'intention du Conseil fédéral, qui, dans le message de 1987, a précisé qu'être "accoutumé» ne signifie pas être "assimilé». Il n'est pas demandé à la personne candidate de renoncer à son identité, mais plutôt de "parvenir à l'issue d'une phase décisive du processus qui aboutira au mariage de ses deux cultures, suisse et étrangère ${ }^{30}$. Dans le cas de $\mathrm{M}^{\mathrm{me}}$ Line, en revanche, la commission communale semble exiger une forme d'assimilation.

M. Paul et $\mathrm{M}^{\mathrm{me}}$ Line font part, lors de l'entretien de recherche de mars 2014, de leur version de l'histoire concernant ce refus. Pour eux, la façon dont $\mathrm{M}^{\mathrm{me}}$ Line s'habille constitue clairement le motif de cette décision:

M. Paul: Ma femme, elle est arrivée en 1977 en Suisse, à l'âge de 10 ans - elle est deuxième génération parce qu'elle est venue avec les parents. Elle a fait ses études ici et puis, en 1998, on a fait une demande de naturalisation ordinaire, 1998, et à ce moment, la commune lui dit "écoutez, vous vous habillez d'une façon islamique» - et ça, j'ai les papiers - "parce que vous êtes influencée par votre mari et donc vous vous habillez comme islamique" - elle s'est convertie à l'islam, c'est normal - «donc nous considérons que vous n'êtes pas intégrée dans la société». 
$\mathrm{M}^{\mathrm{me}}$ Line: Mais la première fois que j'ai demandé, c'est vrai qu'ils m’ont refusé comme quoi je portais le voile et ils m’ont dit que je n'étais pas très adaptée, malgré les nombreuses années que j’ai passées ici en Suisse.

Ce premier résultat négatif entraînant un conflit des visions quant à la signification de l'intégration entre, d'une part, $\mathrm{M}^{\mathrm{me}}$ Line et M. Paul et, d'autre part, l'administration marque le début d'une longue série d'actions de leur part, et de celle d'autres actrices et acteurs impliqués dans la demande. Nous allons analyser ces différentes actions et les différents rôles endossés par les uns et les autres.

\subsubsection{ENGAGER UN AVOCAT}

En 2000, à la suite de ce premier refus, M. Paul prend conseil auprès d'un avocat, qui lui suggère de présenter les certificats scolaires des enfants ainsi qu'un extrait de l'Office des poursuites pour montrer que certains des arguments de la Commission des naturalisations ne sont pas fondés. Par la suite, $\mathrm{M}$. Paul et $\mathrm{M}^{\mathrm{me}}$ Line estimeront inutile l'aide de l'avocat en raison des coûts élevés et du manque d'efficacité de son action:

Chercheuse 1 : Et donc, ily a quelqu'un qui vous a aidés aussi pendant le parcours? Vous avez parlé d'un avocat. [...] Est-ce que vous croyez qu'il a été utile dans la procédure? Qu'il a bien orienté votre... $\mathrm{M}^{\mathrm{me}}$ Line: Pour moi, non! [Rire] Pour moi, non!

M. Paul: C'était le premier. Premier, quand il y avait refus, on avait demandé à cet avocat de faire, mais lui n’avait rien fait.

Chercheuse 2: C'est-à-dire?

M. Paul: C'est juste pour donner l'argent...

$\mathrm{M}^{\mathrm{me}}$ Line: Juste qu'on paye et puis...

M. Paul: Donc la deuxième fois, c'est pour ça, je n'avais pas fait appel à un avocat, j'avais une mauvaise expérience (rire), c'est mieux, je peux faire moi-même! De toute façon, on va chez avocat et il demande ce qu'on doit écrire. Et ça, ça m’est arrivé plusieurs fois. J'étais avec des amis, dans plusieurs cas, moi j'ai dit "mais puisque c'est moi qui dois expliquer ce qu'il doit faire, alors dans ce cas je vais faire moi-même»! ( $\mathrm{M}^{\mathrm{me}}$ Line et $\mathrm{M}$. Paul, mars 2014).

Si M. Paul définit l'avocat comme quelqu'un de peu actif, au contraire, il se présente lui-même comme ayant du savoir-faire 
et étant mobilisé et œuvrant activement pour que la demande de $\mathrm{M}^{\mathrm{me}}$ Line trouve une issue favorable. M. Paul rentre donc dans la catégorie de clients que nous qualifions d'actifs.

\subsubsection{S'ENGAGER DANS LES ÉCHANGES AVEC L'ADMINISTRATION}

M. Paul et $\mathrm{M}^{\mathrm{me}}$ Line sollicitent d'autres acteurs. Ils prennent conseil auprès du Service de la justice qui, selon eux, suggère d'attendre un certain temps avant de déposer une nouvelle demande.

Si c'est $\mathrm{M}^{\mathrm{me}}$ Line qui dépose une deuxième demande de naturalisation en mars 2006 pour elle et ses enfants, c'est son mari qui se positionne comme étant à l'origine de cette initiative. Il n'emploie pas le «nous» lors de son récit, mais le «je»: «En 2006, j’ai fait une nouvelle demande pour ma femme et mes enfants et puis c'était refusé aussi par ce qu'ils disent "vous n'avez pas payé les impôts". Et pourtant javais régulièrement payé les impôts, j"avais un arrangement avec les impôts, que j’ai payés régulièrement» (M. Paul, juin 2013).

En 2009, un nouvel obstacle se présente. Le Service de la justice écrit à $\mathrm{M}^{\mathrm{me}}$ Line pour lui indiquer que sa demande est suspendue, car elle n'est pas à jour dans le paiement des impôts. Dans le courrier, il est précisé qu'elle doit régler ses impôts, ceci relevant du "devoir civique de tous les citoyens", sans quoi la demande de naturalisation sera refusée. Le paiement des impôts est présenté dans le courrier comme étant une des "conditions fédérales à l'octroi de la nationalité suisse» en lien avec le «respect de l'ordre juridique suisse» (extrait du courrier du Service de la justice, août 2009), ceci conformément à l'art. 14 de la loi sur la nationalité de 1990 (let. c).

Comme réaction à la décision du Service de la justice de suspendre la demande de naturalisation, $\mathrm{M}^{\mathrm{me}}$ Line envoie un courrier à ce même service pour expliquer qu' elle a obtenu un arrangement avec l'office de perception qui autorise un paiement échelonné:

J'accuse réception de votre courrier du 19 août 2009 par laquelle nous sommes fortement déçus surtout mes enfants, quand même nous vous en remercions.

Suite d'avoir vérifié mon décompte d'impôt j'ai constaté que vos chiffres mentionnés ne sont pas exacts (justification ci-jointe).

Pour l'année 2007, j'avais un retard de paiement de l'ordre de [...] fr., et me voyant dans l'impossibilité de payer cette grosse somme, j'ai demandé un arrangement à l'office de perception de 
Neuchâtel qui me l'a octroyé. Actuellement, je suis à jour dans le paiement (justifications ci-jointes).

Impôt du 2008 est entièrement réglé (justifications ci-jointes). (Extrait de ce courrier, septembre 2009)

Dans ce premier courrier, $\mathrm{M}^{\mathrm{me}}$ Line écrit à la première personne. Elle exprime tout d'abord sa déception et celle de ses enfants, puis elle conteste l'exactitude des chiffres mentionnés dans le courrier. Elle joint plusieurs documents pour justifier que sa situation financière n’a pas été correctement évaluée par les autorités et pour montrer qu'elle a entrepris les démarches nécessaires concernant le paiement des impôts. Suivant cet accord, elle affirme être à jour dans les paiements. Malgré l'initiative de la cliente, le Conseil d'État l'informe que sa demande de naturalisation est rejetée «en raison du retard dans le paiement de [vos] contributions publiques» (extrait du courrier du Conseil d'État, décembre 2009). Une fois ce critère rempli, elle pourra déposer une nouvelle demande. Les arguments de $\mathrm{M}^{\mathrm{me}}$ Line ne sont pas mentionnés dans la réponse du Conseil d'État.

\subsubsection{FAIRE APPEL À LA JUSTICE}

Vu le manque de résultats à la suite des échanges avec le Service de la justice, $M^{\text {me }}$ Line et $M$. Paul adoptent une autre stratégie en décidant de faire appel à la justice. En janvier 2010, $\mathrm{M}^{\mathrm{me}}$ Line dépose un recours au Tribunal cantonal (Cour de droit public) contre la décision du Conseil d'État du 22 décembre 2009, en demandant son annulation ainsi que l'obtention, pour elle et ses enfants, de la naturalisation. $\mathrm{M}^{\mathrm{me}}$ Line explique dans son recours que cinq mois après avoir reçu «une confirmation de préavis canton de Neuchâtel et fédéral ", soit le 19 août 2009, le "Service de la naturalisation" lui a demandé «de régler entièrement les impôts » de 2007 et 2008 alors qu'elle connaissait une situation économique difficile (extraits $\mathrm{du}$ recours de $\mathrm{M}^{\mathrm{me}}$ Line, janvier 2010). En outre, elle précise que son mari a obtenu le 4 décembre 2009 un arrangement pour le paiement des impôts 2007.

En réponse à ce recours déposé en janvier 2010, le Conseil d'État rédige un courrier pour justifier sa décision négative. Il envoie sa réponse en mars 2010 au Tribunal administratif. $\mathrm{M}^{\mathrm{me}}$ Line est jugée comme n'étant pas en règle avec le paiement des impôts. Le Conseil d'État souligne dans ce même courrier le "pouvoir d'appréciation " dont il dispose dans les décisions de naturalisation et la 
«composante politique forte» de l'acte de naturalisation. Malgré la reconnaissance de fâcheuses lacunes dans l'instruction du dossier, le recours déposé par $\mathrm{M}^{\mathrm{me}}$ Line est rejeté par le Tribunal cantonal (Cour de droit public) le 14 avril 2011. Le paiement des impôts est présenté comme étant une "condition d'aptitude» exigée au niveau cantonal pour obtenir la naturalisation:

Malgré les fâcheuses lacunes dans l'instruction et la tenue du dossier, force est cependant de retenir que la recourante ne remplit pas les conditions d'aptitude, exigées par le gouvernement cantonal pour la naturalisation ordinaire, puisqu'elle reconnaît n'être pas à jour dans le paiement de ses impôts pour l'année 2007. Aussi convient-il d'admettre que la décision attaquée doit être confirmée, ce qui conduit au rejet du recours. (Extrait de l'arrêt du Tribunal administratif cantonal, avril 2011)

Suite à cette décision, $\mathrm{M}^{\mathrm{me}}$ Line fait appel à une instance judiciaire supérieure, le Tribunal fédéral, et dépose un recours constitutionnel subsidiaire le 13 mai 2011. Dans cette démarche, $\mathrm{M}^{\mathrm{me}}$ Line et M. Paul sont aidés par une amie juriste, qui soutient leur action tout au long de la procédure judiciaire sans toutefois jamais apparaître officiellement. Nous rappelons que, en l'absence de droit à la naturalisation, il est possible de recourir contre une décision de refus de la naturalisation seulement en cas de violation des droits constitutionnels (voir chapitre 4). Dans le cas d'espèce, les violations suivantes sont mentionnées: «Le présent recours est formé pour violation des droits constitutionnels, notamment la protection contre l'arbitraire de l'article 9 Cst, la protection de la bonne foi (art. 9 Cst.) et l'interdiction des comportements contradictoires (art. 5 al. 3 Cst. et art. 2 CCS)» (extrait du recours constitutionnel subsidiaire au Tribunal fédéral, mai 2011). Pour fonder son recours, $\mathrm{M}^{\mathrm{me}}$ Line affirme que la décision est motivée d'une façon lacunaire et que les faits ne sont pas soutenus par des preuves:

Dans sa décision du 14 avril 2011, la cour a violé le droit en rendant une décision motivée de manière lacunaire, tout en constatant de manière inexacte et incomplète des faits pertinents.

La Cour dans sa décision a ignoré une partie pertinente de ma vie, tout en retenant l'année de mon mariage comme point de départ de mon intégration dans la société suisse. 
Mon arrivée en Suisse en bas âge (1979) et ma scolarité obligatoire (fin primaire et secondaire 1) me paraît fondamentale pour comprendre le cheminement de mon intégration dans la société suisse, et me donne le droit d'une naturalisation facilitée au sens de l'art. 34 de la convention relative au statut des réfugiés, surtout que je fais partie de la deuxième génération présente en Suisse et que mes copines de l'époque elles sont Suissesses depuis longtemps.

Moyen de preuve:

attestation du 24.08.1979, de l'Office fédéral de la police article 34 de la Convention relative au statut des réfugiés. (Extrait du recours constitutionnel subsidiaire au Tribunal fédéral, mai 2011)

Dans la rédaction du recours, $\mathrm{M}^{\mathrm{me}}$ Line articule des arguments juridiques pertinents, témoignant de son expertise progressive et des arguments du sens commun. En effet, elle emploie des comparaisons, relate les expériences de ses amies comme argument: "Mes copines de l'époque elles sont Suissesses depuis longtemps. » Dans la suite du recours, $\mathrm{M}^{\mathrm{me}}$ Line met en évidence une contradiction présente dans la décision de l'instance inférieure: le Tribunal cantonal a reconnu que la décision du Conseil d'État est lacunaire du point de vue de l'investigation factuelle, mais la décision a tout de même été confirmée. Elle juge cette décision "choquante et arbitraire»:

Pas de preuves des faits rapportés:

La Cour reconnaît dans sa décision [...] qu'aucune pièce du dossier n'établit en outre l'allégation selon laquelle toutes les tranches de l'impôt 2009 facturées étaient en souffrance au moment de la décision attaquée, également, que les dettes d'impôts alléguées par l'intimé ne sont quant à elles que difficilement contrôlables et que le Conseil d'État a estimé inutile de soumettre à la cour de céans des pièces permettant d'établir que des actes de défaut de biens ont été délivrés contre la recourante. Toutefois, la Cour conclut à la confirmation de la décision attaquée [...].

Raisons pour lesquelles je considère sa décision sur la base de ces constatations choquante et arbitraire et par conséquence doit être annulée. (Extrait du recours constitutionnel subsidiaire au Tribunal fédéral, mai 2011)

$\mathrm{M}^{\mathrm{me}}$ Line ajoute aussi des arguments culturels et émotionnels dans son recours pour affirmer la "violation de la bonne foi»: 
son sentiment de confiance vis-à-vis des autorités et de se sentir chez soi a été violé. Ses enfants s'interrogent sur leur futur et sur leur identité :

Moi et mes enfants, on a toujours gardé cette image de la Suisse accueillante, ouverte à l'autre et reconnaissante pour les arrivants qui ceuvrent de leur mieux au bien-être de la Suisse et la société neuchâteloise. Cette envie d'engagement n'est pas étrange pour moi ni pour mes enfants, puisque j'avais 11 ans lorsque je suis arrivée en Suisse (je fais partie de la deuxième génération des immigrés en Suisse), et mes enfants sont nés ici (deuxième génération). Ce sentiment de sécurité, de se sentir chez soi, a été malheureusement touché dans cette affaire, surtout qu'on demande la naturalisation pour la deuxième fois (première procédure en 1998).

Situation qui a marqué la vie de mes enfants les dernières années, mes enfants sentaient depuis une période une déception et se posent la question sur leur identité, citoyenneté et avenir. C'est vrai d'une part, même sans la naturalisation, la Suisse assure une bonne condition de vie, et d'assurances sociales, mais la question est plus profonde, puisqu'elle touche à un sentiment d'appartenance qui se consolidera par la naturalisation. [...]

Violant mes droits constitutionnels, la décision attaquée ne peut dès lors qu'être annulée. (Extrait du recours constitutionnel subsidiaire au Tribunal fédéral, mai 2011)

L'agentivité légale de $\mathrm{M}^{\mathrm{me}}$ Line, qui se fonde sur des arguments juridiques, mais aussi sur le partage de sentiments et d'émotions - élément que nous ne retrouvons pas dans les autres cas et qui est atypique des recours judiciaires -, semble avoir des effets positifs: le 27 septembre 2011, le Tribunal fédéral admet le recours en affirmant qu' 'il n'est pas possible de refuser la naturalisation de la recourante sur la base de données aussi lacunaires» et renvoie l'affaire à l'instance inférieure (la Cour cantonale) pour nouvelle décision. Le 24 janvier 2012, la Cour cantonale - dans sa décision - reconnaît aussi des lacunes dans la procédure de constatation de faits par la Commission des naturalisations et annule la décision, viciée à plusieurs égards :

Le Conseil d'État dispose d'un large pouvoir d'appréciation en matière de naturalisation. La procédure de naturalisation ne se déroule 
toutefois pas dans un cadre dépourvu de toutes règles juridiques. L'autorité doit faire usage de son pouvoir d'appréciation - même s'il est très large, en respectant ses devoirs et en observant le sens et le but de la législation sur la naturalisation. [...] Il lui incombe aussi de motiver ses décisions [...].

De la sorte, il apparaît que la commission chargée de l'examen préalable des dossiers de naturalisation ne disposait pas de renseignements exacts sur le cas de la recourante lorsqu'elle s'est déterminée dans ses séances des 25 juin et du 9 décembre 2009. La constatation des faits par l'autorité intimée se révèle ainsi inexacte (art. 33 let. b LPJA) [...].

Il suit des considérants qui précèdent que la décision entreprise découle d'une procédure gravement viciée à plus d'un égard. Elle doit être annulée et la cause renvoyée au Conseil d'État pour qu'il reprenne l'instruction du cas, dans le respect des garanties de procédure offertes à la recourante par la Constitution et les lois, avant de statuer à nouveau par une décision dûment motivée.

Le Conseil d'État est ainsi prié d'examiner à nouveau la demande de $\mathrm{M}^{\mathrm{me}}$ Line. Alors que les autorités judiciaires, à la suite du recours admis, ont demandé un nouvel examen, $M^{\text {me }}$ Line ne reçoit pas de nouvelles. De ce fait, en mai 2013, elle adresse un courrier au Service de la justice afin de connaître le statut de sa demande tout en évoquant son droit de faire des démarches pour dénoncer le déni de justice: "Je vous prie de bien vouloir me faire parvenir votre décision finale jusqu'au 03.06.2013 au plus tard, à défaut je serai dans obligeance de faire démarches auprès de l'autorité compétente contre déni de justice selon l'art. 46a» (extrait du courrier de $\mathrm{M}^{\mathrm{me}}$ Line au Service de la justice).

Suite à un nouvel examen de la situation, le Conseil d'État estime que les conditions pour la naturalisation ne sont toujours pas remplies, en argumentant de nouveau que pour obtenir la naturalisation neuchâteloise, les candidats doivent respecter l'ordre juridique suisse, et de ce fait payer leurs impôts dans leur totalité. Ainsi, l'obstacle majeur à l'obtention de la naturalisation pour $\mathrm{M}^{\mathrm{me}}$ Line s'avère être celui des obligations financières dont elle doit répondre solidairement avec son époux. C'est sur la base des arguments de la Commission cantonale chargée de l'examen préalable des dossiers de naturalisation qui avait constaté que "toutes les dettes envers l'État n'avaient pas été réglées" que le Conseil d'État décide de 
«refuser la naturalisation neuchâteloise selon forme ordinaire». $\mathrm{M}^{\mathrm{me}}$ Line est ainsi invitée à remplir les conditions légales afin de pouvoir déposer une nouvelle demande: «Si votre désir d'obtenir la naturalisation neuchâteloise subsiste, il vous appartient de déposer une nouvelle demande, dès que les conditions vous sembleront remplies» (pour toutes ces citations, voir l'extrait du courrier du Conseil d'État, juillet 2014).

$M^{\text {me }}$ Line saisit à nouveau le Tribunal cantonal d'un recours contre la décision du Conseil d'État, dont elle demande l'annulation. La Cour, par arrêt du 24 novembre 2015, admet le recours et le renvoie à l'autorité inférieure pour nouvelle décision. Parmi les motivations de la Cour figurent les indications suivantes: "le manque de rigueur dans la présentation de la situation financière de la recourante»; "la décision contestée ne contient pas les éléments de fait déterminants, ne satisfait pas aux exigences de motivation". À nouveau, la procédure du Service de la justice est considérée comme viciée: "Pour les raisons évoquées ci-dessus, il y a lieu de considérer que la décision entreprise découle encore une fois d'une procédure viciée pour plusieurs raisons. Il convient de l'annuler et de renvoyer la cause au Conseil d'État pour instruction complémentaire» (extrait de l'arrêt du Tribunal cantonal, novembre 2015).

En août 2016, M ${ }^{\mathrm{me}}$ Line obtient la nationalité grâce à la décision du Service de la justice de distinguer ses obligations de celles de son mari. Bien que nous ne possédions pas les documents du dossier administratif correspondant à cette dernière étape de la procédure, la conclusion de l'histoire a été racontée par $\mathrm{M}^{\text {me }}$ Line à un membre de notre équipe de recherche, à qui elle a montré son passeport avec une photo d'elle portant le voile.

\subsubsection{UNE AGENTIVITÉ PARTAGÉE}

Nous pouvons identifier trois modalités d'action dans la trajectoire administrativo-légale de $\mathrm{M}^{\mathrm{me}}$ Line: 1) s'engager dans les échanges avec l'administration (se renseigner auprès des autorités dans le but d'instaurer un dialogue, être active dans la recherche d'informations pertinentes pour la solution de son cas); 2) engager un avocat et renoncer à l'aide de l'avocat, ses services étant jugés peu utiles et trop onéreux; 3) faire appel à la justice (activer la justice pour faire reconnaître ses droits vis-à-vis de l'administration). 
L'agentivité de $\mathrm{M}^{\mathrm{me}}$ Line a la particularité d'être partagée avec son mari. Bien qu'elle soit la candidate de la procédure de naturalisation, son mari est partie prenante dans ses démarches. Au-delà de l'engagement de celui-ci, l'évaluation de la demande de $\mathrm{M}^{\mathrm{me}}$ Line est aussi liée à la situation financière de son mari: ils sont solidairement responsables des obligations financières. Leur agentivité est partagée aussi dans le cercle restreint d'amies et d'amis, en particulier avec une amie juriste qui les aide à l'occasion des différents recours. Les différentes modalités d'action combinées au fil du temps permettent à $\mathrm{M}^{\mathrm{me}}$ Line d'obtenir la nationalité près de vingt ans après le début de la première procédure.

En fait, nous observons l'agentivité croissante de $\mathrm{M}^{\mathrm{me}}$ Line en tant qu'actrice étrangère pendant les deux procédures de naturalisation. À la suite du premier refus (1998), un conflit prend forme entre les représentations et les attentes des autorités suisses relatives à la façon de $\mathrm{M}^{\mathrm{me}}$ Line de se présenter à la Commission communale. Pour la commission, les motivations de $\mathrm{M}^{\mathrm{me}}$ Line sont génériques et mal définies: celle-ci avance des arguments de la vie quotidienne (sentiments d'incertitude pour son futur et l'avenir de ses enfants, etc.) visant à convaincre de lui accorder la naturalisation. À l'occasion de la deuxième procédure, $\mathrm{M}^{\mathrm{me}}$ Line agit en accord avec le droit et fait face au système administratif en utilisant des moyens légaux et juridiques pertinents, ou attendus, pour démontrer aux autorités que l'évaluation de son cas et de son intégration est défaillante.

Cette appropriation progressive du système, des règles formelles et des codes du pays d'accueil, témoignant de sa conscience juridique et culturelle, permet à $\mathrm{M}^{\mathrm{me}}$ Line, et aux personnes dont elle s'entoure dans cette démarche, de comprendre comment agir visà-vis des institutions - quelles autorités solliciter, quels moyens utiliser - ou encore de définir la nécessité ou non de consulter un avocat. Cette agentivité se matérialise à travers les différents documents adressés par $\mathrm{M}^{\mathrm{me}}$ Line aux autorités: un langage de plus en plus expert et formel est utilisé à mesure que la procédure avance. La loi est évoquée d'une façon appropriée pour légitimer les demandes vis-à-vis des différentes instances interpellées.

Les différentes actions entreprises par $\mathrm{M}^{\mathrm{me}}$ Line auprès de l'administration et de la justice et les différentes réactions des instances judiciaires, en particulier la décision du Tribunal fédéral de 2011 et ensuite celles du Tribunal cantonal, ont provoqué aussi 
des modifications dans le cadre de la procédure de naturalisation au niveau cantonal. Le dossier de naturalisation construit par le Service de la justice doit maintenant contenir toutes les données relatives à la personne candidate (selon les informations obtenues lors des entretiens et observations menés dans ce service).

\subsection{DIFFÉRENTES MODALITÉS D'AGENTIVITÉ}

Dans les lignes qui suivent, nous présentons de façon plus synthétique les autres cas, ceux de MM. Charles, Kirin, François et Johan, dans le but d'analyser comment s'articulent dans ces parcours les trois niveaux d'agentivité que nous avons retracés dans le cas de $\mathrm{M}^{\mathrm{me}}$ Line, soit: s'engager dans les échanges avec l'administration; engager un avocat; faire appel à la justice. Ces trois niveaux sont toujours analysés en lien avec le positionnement du client vis-à-vis du droit et de la procédure (face au droit, avec le droit, contre le droit).

\subsubsection{UNE AGENTIVITÉ SUSPENDUE: LE CAS DE M. CHARLES}

En novembre 2003, dix ans après son arrivée en Suisse en tant que requérant d'asile, $M$. Charles dépose une demande pour obtenir la nationalité suisse. Deux préavis négatifs de la part du Conseil communal empêchent $M$. Charles d'obtenir l'autorisation fédérale de naturalisation, préalable à l'obtention du droit de cité neuchâtelois. Plus de huit ans après le dépôt de sa requête, $M$. Charles abandonne la procédure et renonce, du moins provisoirement, à la naturalisation (chapitre 6).

Nous précisons que les conditions pour l'octroi de la nationalité étaient, dans le cas de M. Charles, les mêmes que pour le cas de $\mathrm{M}^{\mathrm{me}}$ Line et indiquées à l'art. 14 de la loi sur la nationalité de 1990 (let. a-d). C'est ainsi que M. Charles motive sa demande en répondant aux critères d'intégration demandés par la loi. Il met spécialement l'accent sur sa maîtrise du français, sur l'apprentissage de son métier ainsi que sur sa participation à la vie sociale et culturelle du pays (voir extrait de la demande de naturalisation, chapitre 6, 6.2.2).

Toutefois l'autorisation fédérale de naturalisation lui sera refusée par le Secrétariat d'État aux migrations en raison de «doutes» sur son intégration, formulés par les autorités communales et cantonales sur la base d'un rapport de la police: 
Monsieur,

Nous référant à votre demande d'autorisation fédérale de naturalisation (art. $13 \mathrm{LN}$ ), nous devons vous faire savoir que les autorités cantonales consultées ont émis un préavis négatif à l'endroit de votre requête. Elles font en particulier valoir qu'elles doutent de votre intégration dans notre pays.

Comme dans ces conditions, une naturalisation sur le plan cantonal paraît hypothétique, nous vous prions de nous faire savoir si vous entendez poursuivre la procédure ou si vous retirez votre requête. Sans réponse de votre part dans un délai d'un mois, par écrit, nous classerons votre demande comme étant devenue sans objet. (Extrait du courrier du Secrétariat d'État aux migrations du 12 mai 2006)

Nous allons reconstruire le cas de M. Charles autour de ses réactions vis-à-vis des réponses des autorités. Tout d'abord, moins d'un mois après la réception du courrier évoqué, $M$. Charles écrit au Secrétariat d'État aux migrations pour faire part de son «étonnement» quant au préavis négatif et au motif avancé par les autorités cantonales. Nous reproduisons ce courrier dans son intégralité:

Madame, Monsieur,

J'ai lu avec beaucoup d'étonnement votre courrier m'annonçant que les autorités cantonales ont émis un préavis négatif concernant ma demande de naturalisation avec le motif qu'elles doutent de mon intégration en Suisse. La personne chargée d'étudier les dossiers dans l'administration communale m'a informé de sa part que leur doute est basé essentiellement sur le fait que je n'avais pas une situation professionnelle stable. [...] Je me suis trouvé en effet sans activité durant environ une semaine en attendant le début d'un nouveau contrat de travail. Je me permets de préciser qu'il s'agit de la seule période sans activité que j’ai connue depuis longtemps. Je ne connaissais pas le fait qu'un phénomène socio-économique comme le chômage, même de très courte période, soit susceptible de générer des doutes en ce qui concerne l'état de l'intégration d'une personne.

Je suis arrivé en Suisse à 16 ans. J'y vis depuis plus de treize ans. Après deux ans de classes d'accueil qui m'a permis d'apprendre le français, j'ai commencé à travailler dans l'horlogerie en tant qu'apprenti polisseur. Depuis, je travaille dans ce domaine en tant que polisseur. En raison des fluctuations du domaine de l'horlogerie, j'ai été au chômage pour des courtes périodes, mais j’ai toujours 
travaillé depuis mes 18 ans. Je travaille actuellement avec un contrat de durée indéterminée toujours dans le même domaine. Toutes mes relations sociales, mes relations d'amitié et de solidarité se trouvent en Suisse et elles sont nombreuses. Je fais partie de plusieurs clubs locaux et je participe activement à la vie sociale, culturelle et politique de mon canton. Je n'ai pas d'antécédents ni de casier judiciaire. Il n'existe pas de poursuites me concernant. Je suis financièrement autonome et en règle en ce qui concerne l'imposition communale, cantonale et fédérale.

C'est pourquoi j'estime le préavis des autorités cantonales excessivement injuste à l'endroit de ma demande de naturalisation. Je trouve en effet qu'il est injuste de qualifier de douteuse l'intégration professionnelle d'une personne parce qu'elle s'est trouvée au chômage indépendamment de sa volonté et pour une courte période en raison de l'évolution des conditions économiques.

Je pense sincèrement que les autorités cantonales et communales, qui nous ont donné des exemples formidables d'ouverture à l'égard de ses concitoyens issus des migrations, vont réexaminer le préavis en question.

Je vous envoie en annexe quelques documents qui attestent ma situation juridique économique et professionnelle, et vous prie donc de bien vouloir prendre note que je désire poursuivre la procédure en vue de la naturalisation

Dans l'attente de votre confirmation, veuillez agréer, Madame, Monsieur, mes salutations distinguées. (Courrier de M. Charles, juin 2006)

Dans ce courrier, les arguments développés par M. Charles, documents à l'appui, prouvent selon lui que la décision est injuste et que sa demande doit être examinée à nouveau.

En ce point de sa trajectoire administrative et de vie, M. Charles se sent légitimé à demander la citoyenneté et est en mesure de dire à la fin de son courrier que les autorités «vont réexaminer le préavis en question». Il agit en accord avec le droit.

Nous pouvons ainsi identifier plusieurs niveaux d'agentivité dans la réponse de $\mathrm{M}$. Charles en tant qu'acteur non expert du droit, mais capable d'initiative ${ }^{31}$ : il met en avant sa capacité à se tenir informé, à mobiliser des actrices et acteurs pour avoir des réponses;

31. Di Donato, 2008a, 2008b; Haavisto, 2002. 
il justifie sa situation personnelle et professionnelle par la crise économique plus générale; il reconstruit son parcours professionnel afin de démentir le fait que sa vie professionnelle ne serait pas stable et de démontrer sa volonté de travailler depuis son arrivée en Suisse.

La requête de M. Charles est ainsi acceptée. En août 2008, un rapport de police est rédigé avec une conclusion nettement plus positive: "M. Charles nous paraît être une personne équilibrée et bien établie sur sol neuchâtelois. Malgré qu'il ouvre la énième pizzeria/kebabs, il montre des prétentions concernant son avenir professionnel.» Toutefois, malgré le résultat positif de cette deuxième évaluation, le Conseil communal formule à nouveau un préavis négatif. Il n'est plus question alors d' «instabilité» ou de "doutes" sur son intégration, mais, cette fois-ci, le Conseil communal estime que M. Charles n'a pas payé la totalité de ses impôts et qu'il est "connu des services de police» (extrait du préavis du Conseil communal, septembre 2008). Il ne remplit dès lors pas un des critères de l'art. 14 de la loi sur la nationalité de 1990 (let. c), c'est-à-dire le respect de l'ordre juridique suisse.

Confronté à ces obstacles dans sa démarche administrative, M. Charles fait appel à un avocat, et ceci pour deux objectifs distincts: inclure sa femme dans la demande et avoir accès à son dossier. L'avocat rédige en ce sens un courrier au Service de la justice. Ce dernier répond à l'avocat de $\mathrm{M}$. Charles que la femme de celui-ci peut être intégrée à la demande en cours de procédure. Dans le même courrier, il est précisé que les informations obtenues dans le cas de M. Charles ne permettront «vraisemblablement pas de donner un avis favorable» quant à sa demande (courrier du Service de la justice, octobre 2008). L'avocat transmet cette réponse à $\mathrm{M}$. Charles en indiquant dans les détails les démarches pour intégrer sa femme dans la procédure malgré le fait que le courrier du Service de la justice laisse entendre que les chances de M. Charles d'obtenir la naturalisation sont limitées.

M. Charles sollicite à nouveau son avocat afin d'obtenir son dossier. L'avocat contacte le Service de la justice en novembre 2008 afin de connaître les éléments sur lesquels le préavis négatif se fonde. Lorsque l'avocat reçoit le dossier du Service de la justice, en mai 2009, soit près de six mois après la requête, il le transmet à $\mathrm{M}$. Charles en le commentant. Il explique que le refus est dû à deux facteurs: «l'existence des poursuites en cours» et le fait qu'il soit «connu» des services de police (extrait du courrier de l'avocat, 
mai 2009). Dans la suite de son courrier, l'avocat donne des indications plus détaillées concernant la situation de $\mathrm{M}$. Charles, tout en invitant son client à consulter les documents. L'avocat joue le rôle de traducteur-médiateur entre les pratiques de l'administration et son client ${ }^{32}$. Il lui demande aussi des renseignements par rapport à des faits dont il n'est pas informé:

Je vous laisse prendre connaissance de ces annexes. S'agissant de la question des Services de police, j’entends me prévaloir du jugement que nous avons reçu du Tribunal de police, qui est favorable.

Vous constaterez qu'il y a également un rapport de police pour une affaire d'injures et de menaces remontant à l'année 2008. Je ne connais pas ce dossier et vous laisse le soin de mindiquer ce quill en est à cet égard. Pour moi, ce qui comptait essentiellement était le contenu du casier judiciaire, qui ne comporte, à ma connaissance, aucune indication à cet égard.

Enfin, il conseille à $\mathrm{M}$. Charles de radier ses poursuites en engageant des pourparlers avec le Service de la justice et avec les créanciers:

En ce qui concerne les poursuites et l'existence d'arriérés d'impôts, il y aurait lieu, pour activer le dossier, que les poursuites soient radiées, et que vous trouviez un arrangement avec le Service des contributions. À cet égard, il me paraît nécessaire d'engager des pourparlers avec le Service précité, ainsi qu'avec vos créanciers. (Extrait du courrier de l'avocat, mai 2009)

Il s'agit ainsi de continuer à agir en accord "avec le droit» et le système. M. Charles pourra s'occuper lui-même des démarches ou donner mandat à son avocat en lui versant une provision supplémentaire. Similairement à $\mathrm{M}^{\mathrm{me}}$ Line, n'étant pas satisfait du travail de l'avocat et en estimant le coût trop élevé, M. Charles se dispense de ses services, comme il en témoigne dans l'entretien de recherche (juin 2014) :

J'ai suivi avec un avocat un certain temps, cétait trop cher. Et puis j'ai vu que aussi qu'il ne me rendait pas trop de services parce qu'il écrivait 
juste la lettre pour rassembler certains documents et une fois qu'il a rassemblé je crois qu'il a fait recours, moi j'ai arrêté avec l'avocat, j’ai dit «Bon, je suis mon dossier tout seul».

En juin 2011, M. Charles adresse lui-même un courrier aux autorités. Dans ce courrier, il reprend les points suggérés par l'avocat dans leur dernier échange afin de convaincre les autorités qu'il remplit les critères d'intégration. $M$. Charles déclare «faire un point d'honneur de s'intégrer le plus possible» dans la vie sociale et professionnelle de sa région (et de «respecter les règles de la circulation routière») :

Comme vous le savez, jai engagé une procédure de naturalisation, il y a quelque temps. Je souhaite vous faire part des développements intervenus dans ma situation personnelle et familiale.

En premier lieu, je vous informe que je me suis remarié, le [...] devant l'Officier de l'État civil [de la commune], avec Madame [...]. Vous trouverez en annexe copie du certificat de famille délivré par l'Office de l'État civil.

Je suis divorcé d'avec mon ancienne épouse selon divorce devenu définitif et exécutoire le 26 mai 2010. À cet effet, je vous joins copie du livret de famille, avec mention de l'Office de l'État civil [de la commune].

Sur le plan financier, je vous confirme que jai toujours une activité salariée à quelque $100 \%$, auprès de l'entreprise [...], où je réalise un salaire mensuel brut de $5000 \mathrm{fr}$.

Vous trouverez en annexe copie d'une fiche de salaire récente.

Par ailleurs, j'exploite également la Pizzeria [...] avec mon épouse, en vue de développer cette activité qui nous l'espérons sera pourvoyeuse d'emploi.

Ma situation financière est tout à fait saine.

Je n'ai aucune poursuite, ce qui ressort de l'extrait de l'Office des poursuites était dî̀ à des problèmes comptables occasionnés par le début de notre activité de notre pizzeria et aussi par le problème du divorce de mon ex-épouse.

Je me fais un point d'honneur de m'intégrer le plus possible dans la vie sociale et professionnelle de notre région.

Comme vous le savez, je suis membre de la Commission de sécurité de la Ville de [...] depuis quelques années et depuis la fin de l'été 2009, j’ai également rejoint le Conseil général de la Ville de [...] 
Je précise, en lien avec le rapport de naturalisation établi par la Police cantonale [de la commune], en date du 13 août 2008, que l'épisode lié à un accident de la circulation est définitivement réglé.

Il s'agit d'un fait isolé.

Je précise que cette affaire s'était réglée par une transaction passée le 18 mai 2008 (amende de 250 fr.).

Comme indiqué, cette affaire est isolée, et je me fais un point d'honneur de respecter les règles de la circulation routière.

Aussi, je vous saurais gré de bien vouloir aller de l'avant dans la procédure de naturalisation en cours et de faire le nécessaire pour accélérer la procédure de naturalisation.

Je reste bien entendu à votre entière disposition pour tout complément d'information ou un entretien éventuel.

Veuillez agréer, Mesdames, Messieurs, l'expression de mes sentiments distingués. (Extrait du courrier de M. Charles, juin 2011)

Entre-temps, le Service de la justice lui répond que sa demande a été archivée dans l'intervalle, mais qu'il peut s'il le souhaite déposer une nouvelle demande.

Tout au long de la trajectoire administrativo-légale de M. Charles, nous pouvons identifier trois types d'actions. 1) Se positionner face au droit: faire les démarches de naturalisation et s'engager à prouver son intégration auprès des autorités. 2) Être avec le droit: engager un avocat et renoncer à l'aide de l'avocat, qui ne se révèle pas être utile et qui est trop cher à ses yeux. En fait, si les buts pour lesquels M. Charles s'adresse à son avocat ne sont pas clairement définis (intégrer sa femme dans la procédure et demander son dossier), l'action de l'avocat semble néanmoins s'être limitée à la requête d'informations à l'administration et à la traduction de ces informations en conseils. De son côté, M. Charles omet d'informer l'avocat d'un détail de son parcours qui s'avère significatif dans l'évaluation de l'intégration: une affaire de police qui aurait contribué au refus. Nous pouvons en déduire que l'interaction entre M. Charles client et son avocat n'est pas construite sur une base de confiance, ce qui est primordial pour la réussite d'une stratégie légale ${ }^{33}$. 3) Etre contre le droit, voire renoncer au droit lorsque $\mathrm{M}$. Charles n'arrive plus à suivre la logique de l'administration. Les obstacles rencontrés, comme les préavis négatifs ou encore les coûts élevés pour les frais d'avocat,

33. Ellmann et al., 2009. 
font que M. Charles n'est plus avec le droit. M. Charles - de la même manière que M. Paul - affirme qu'il existe de nombreuses "contradictions» dans la loi qui lui déplaisent. Enfin, il ne souhaite pas «jouer» avec ces règles et suspend sa démarche.

\subsubsection{UNE AGENTIVITÉ PLANIFIÉE: LE CAS DE M. KIRIN}

Vingt-quatre ans après son arrivée en Suisse en tant que requérant d'asile, M. Kirin dépose sa demande de naturalisation, puisqu'il se sent "bien intégré et bien assimilé». Il estime que c’est aussi un "devoir de servir ce pays» qui l'a accueilli et qui lui a "permis de vivre en sécurité» (extraits de la demande de naturalisation, juin 2005). Comme pour $\mathrm{M}^{\mathrm{me}}$ Line et $\mathrm{M}$. Charles, des impayés vont entraver sa demande.

Nous allons reconstruire certaines des étapes de l'histoire de M. Kirin pour mettre en évidence ses propres modalités d'action et ses positionnements par rapport au droit et à l'administration: en agissant seul ou avec l'aide d'un ou d'une avocate, ou encore en combinant les deux façons d'agir.

Dès le début de la procédure de naturalisation, M. Kirin sollicite différentes personnes pour l'aider ou obtenir des renseignements. Il explique lors de l'entretien de recherche (juin 2015) qu'il a demandé des informations auprès de sa commune:

Moi je suis d'abord passé à la commune une fois, pour me renseigner. Ils m’ont présenté un formulaire. Un formulaire qui demandait pas mal de choses. On me demandait beaucoup sur, par exemple, le, comment ça s'appelle... le casier judiciaire... Ils me demandaient sur les impôts, ils demandaient... c'est comme ça que je me suis renseigné.

En octobre 2004, M. Kirin donne mandat à une avocate en vue des démarches à faire. L'avocate dépose la demande de naturalisation au Service de la justice le 14 juin 2005. En février 2006, soit à peu près huit mois après l'ouverture du dossier, un échange de plusieurs courriers commence entre l'avocate de M. Kirin et le Service de la justice. L'avocate sollicite le Service de la justice à cinq reprises: la première fois, soit le 21 février, elle demande d'indiquer le «sort» de la procédure. Le lendemain, le Service de la justice répond à l'avocate que "les démarches pour une telle demande sont très longues ". Toutefois, ils s'efforceront de faire le plus rapidement possible et comptent sur leur "compréhension» (extraits du courrier 
du Service de la justice, février 2006). La deuxième fois, l'avocate demande "d'indiquer où en sont les démarches" (novembre 2006) et elle n'obtient pas de réponse. La troisième fois, elle demande au Service de la justice de l' "informer de l'évolution de ce dossier", puisque son courrier du mois de novembre 2006 n'a pas reçu de réponse (février 2007). Le Service de la justice répond et justifie le retard "dans le traitement des dossiers" en évoquant "une restriction du personnel» (extraits du courrier du Service de la justice, février 2007). Dans la quatrième requête, du mois de janvier 2008, soit onze mois après son dernier courrier et plus de deux ans après l'ouverture du dossier, l'avocate demande une accélération de la procédure, car le délai usuel pour une procédure de naturalisation a été dépassé. De plus, l'avocate argumente la demande d'accélération de la procédure par le fait que son client a des exigences concrètes, comme la nécessité d'obtenir une pièce d'identité pour se rendre à l'étranger. Il s'agit d'une exigence formulée par d'autres candidats, comme $\mathrm{M}^{\text {me }}$ Line ou $\mathrm{M}$. Bruno (voir chapitre 6). Lavocate utilise aussi un argument qui porte sur ce qui est souvent défini comme l'«intégration culturelle» pour convaincre les autorités que M. Kirin remplit les critères demandés pour obtenir la naturalisation.

Etant totalement assimilé à notre pays, où il vit depuis plus de vingtcinq ans, il aspire enfin à pouvoir en acquérir la nationalité. On est en droit de considérer que ce cas mérite d'être traité rapidement, n'ayant rien à voir avec les cas de personnes qui devenant doubles nationaux peuvent conserver avec leur pays d'origine des relations, ce qui n'est pas le cas de mon client qui a demandé l'asile politique. Je vous remercie dès lors de tout faire pour que l'autorisation requise puisse être envoyée rapidement à mon client. (Extrait du courrier de l'avocate au Service de la justice, janvier 2008)

L'avocate ne se limite pas à parler d'intégration - terme utilisé dans la loi -, mais parle dans ses courriers au Service de la justice d'assimilation, en évoquant un critère qui est toutefois dépassé sur le plan de la formulation législative (voir chapitres 3 et 4). Comme nous l'avons vu pour le cas de $\mathrm{M}^{\mathrm{me}}$ Line, la loi sur la nationalité dans sa version de 1990 ne demande plus l'assimilation des candidats, mais leur familiarisation avec les coutumes et les usages suisses comme étape du processus d'intégration. L'avocate souligne aussi que son client, à la différence des autres personnes étrangères, n’a pas gardé 
sa nationalité d'origine et n'a pas de relations avec son pays, raisons pour lesquelles il a d'autant plus besoin de la naturalisation.

En avril 2008, l'avocate envoie une dernière requête au Service de la justice. Son courrier croise celui du Service de la justice, envoyé le même jour. Le Service de la justice "présence ses excuses" et "regrette la situation", en précisant toutefois qu'un délai de dixhuit mois est encore nécessaire pour traiter le dossier (extraits du courrier du Service de la justice, avril 2008). À fin avril 2008, soit plus de quatre ans après avoir mandaté une avocate et plus de trois ans après le dépôt de sa demande, $M$. Kirin rentre alors en scène et prend l'initiative d'écrire directement au chef du Département de la justice afin d'obtenir des informations sur le suivi de son dossier. La lettre est manuscrite; en voici la retranscription:

Concerne: ma demande de naturalisation.

Monsieur,

Je viens madresser auprès de votre autorité, pour vous affirmer combien je suis choqué de la longueur extrême de la procédure de ma naturalisation.

Ma demande de naturalisation remonte depuis 2005. En 2007, par le biais de mon avocat Maître $\mathrm{X}, \mathrm{j}$ 'ai reçu une réponse de la part de votre dépt. me demandant de patienter (voir copie).

Depuis jai attendu jusqu'en 2008 pour relancer votre dépt., et je reçois à nouveau une réponse où l'on me demande d'abord de payer 350 fr. du décret du $1^{\text {er }}$ février 2006, et ensuite de devoir encore patienter pour un délai d'environ 18 mois soit une année et demi.

Je viens de payer la facture de 350 fr. (voir copie récépissé) et je me demande encore pourquoi je devrais attendre si longtemps (dix-huit mois) pour une demande qui a été faite en 2005 ???

Vous écrire c'est aussi vous demander une vue voir un regard juste en ce qui concerne cette demande de naturalisation.

Dussé-je me sentir devenir une victime d'une attente aussi longue avec comme seule justification un manque d'effectifs dans votre département, je trouve que c'est un peu facile comme raison.

Sur ce, j'aurais souhaité obtenir un entretien avec vous afin de m'éclairer sur ma situation.

Je vous présente, Monsieur, mes meilleures salutations.

(M. Kirin, signature)

(lettre manuscrite de M. Kirin adressée au Chef du Département de la justice - 28 avril 2008) 
Dans ce courrier peu formel, des abréviations sont utilisées (dépt.) ainsi que des points d'interrogation (???). M. Kirin, similairement à $\mathrm{M}$. Charles et à $\mathrm{M}^{\mathrm{me}}$ Line, affirme être "choqué de la longueur extrême de la procédure", liée apparemment au manque de personnel de l'administration. Cette raison lui semble " un peu facile» et il n'hésite pas à demander un entretien. Il revendique « un regard juste» sur sa demande (extraits du courrier de M. Kirin, avril 2008).

En mai 2008, le chef du Département de la justice répond en personne à $\mathrm{M}$. Kirin et en copie à son avocate. Il indique que le «retard est dû certes à un manque de personnel au service de la justice, mais également à la complexité de traitement des demandes de naturalisation ". En conclusion, si M. Kirin souhaite obtenir la nationalité suisse, "il doit faire preuve d'encore un peu de patience».

En septembre 2008, M. Kirin reçoit des informations du Service de la justice quant à sa demande: le préavis communal est négatif. Notons qu'il s'est écoulé cinq mois depuis que le Service de la justice a demandé à la police de présenter un rapport. Le courrier indique qu'il "ressort" du rapport d'enquête qu'il n'est "pas en règle dans le paiement de ses contributions fiscales". Il lui est aussi recommandé de "retirer sa requête" au "vu de sa situation». Toutefois, si les impôts peuvent être réglés dans les six mois, le Service de la justice précise être «disposé à poursuivre la procédure». M. Kirin doit informer de sa décision dans le délai de trente jours. M. Kirin réagit. Il écrit directement à la personne responsable du Service de la justice, pour indiquer qu'il a entrepris "une démarche de désendettement», et ce, depuis 2002. Dans son courrier, il mentionne l'aide du Centre social protestant probablement pour légitimer son action ${ }^{34}$. Il demande au Service de la justice de poursuivre la procédure en assurant qu'il fait tout ce qui est en son pouvoir "pour être un citoyen et un contribuable honnête».

La forme de ce courrier, dactylographié, differe de la première lettre, manuscrite, envoyée par M. Kirin le 28 avril 2008 au chef du Département de la justice. La présentation et le style sont soignés. Des tournures formelles sont utilisées. Voici la lettre retranscrite dans son intégralité:

34. Le Centre social protestant est une association privée qui offre un service de consultation et d'assistance sociale et juridique. 
Recommandée

Service de la Justice À l'att. de xxx

Château

2001 Neuchâtel

Le 22 Sept. 08

Demande de naturalisation

Votre référence $\mathrm{xxx}$

Madame,

Par la présente, je donne suite à votre courrier du 3 ct. qui a retenu ma meilleure attention. À cet égard, je tiens à vous préciser que j’ai entrepris avec le soutien du Centre social protestant (CSP), une démarche de désendettement, en 2002 déjà. Au prix de nombreux efforts dans la gestion de mon budget et également en procédant à d'importants sacrifices financiers, je suis venu à bout de mes dettes après quatre années.

Cependant, suite à des changements répètes d'activité professionnelle et donc de revenus (chômage, travail intérimaire, etc.), puis un mariage et ensuite une séparation. Les montants à payer pour mes impôts n'ont pas cessé de changer ces dernières années (et même en cours d'année). C'est donc pour des raisons indépendantes de ma volonté que je me suis trouvé dans cette situation. Si bien que j'ai actuellement un arrangement (cf. copie en annexe), avec l'office de perception pour un solde d'impôts 2006 que je terminerai de régler en janvier 2010. Les impôts courants sont eux à jour, c'est d'ailleurs une condition de l'arrangement précité.

En fonction de ce qui précède, je peux vous assurer que je fais tout ce qui est en mon pouvoir pour être un citoyen et un contribuable honnête. C'est pourquoi je vous saurais gré de bien vouloir continuer la procédure pour ma demande de naturalisation, malgré cet arrangement de paiement.

En effet, je suis toujours soutenu par le CSP, XXX avec qui je gère l'ensemble de mes factures et je me tiens à jour dans ma compatibilité. Je reste volontiers à votre disposition pour tout complément d'information et vous remercie par avance de l'attention portée à ma demande.

Dans l'attente de vos nouvelles, je vous adresse, Madame, mes salutations les meilleures.

(M. Kirin, signature)

(lettre dactylographiée de M. Kirin adressée au Service de la justice - 22 septembre 2008) 
En février 2009, la personne responsable du Service de la justice envoie sa réponse à l'avocate, bien que le courrier ait été envoyé par M. Kirin. Malgré les informations à propos de l'arrangement de désendettement, le préavis négatif reste inchangé. Le dossier est toutefois transmis au Secrétariat d'État aux migrations en vue de l'obtention de l'autorisation fédérale de naturalisation. Le courrier du Service de la justice précise que «si l'autorisation de naturalisation est délivrée, cela n'engage pas une décision communale et cantonale favorable" (extrait du courrier du Service de la justice à l'avocate de M. Kirin, février 2009). Dans les deux mois qui suivent, l'avocate répond au Service de la justice, sans mentionner d'interlocuteur spécifique, et demande à obtenir le préavis négatif qu'elle n'a jusqu'alors jamais reçu. Elle recevra le dossier de M. Kirin en mai 2009.

Tout au long de la démarche de M. Kirin, nous pouvons identifier trois modalités d'actions. 1) Être face au droit: au début de la procédure, M. Kirin, comme M. Charles, croit au droit et à sa rationalité. Il s'engage à comprendre la procédure de naturalisation. 2) Être avec le droit: confronté à la complexité de la demande de l'administration, M. Kirin s'adresse à une avocate dans le but de bien réussir dans sa démarche. Son avocate se révèle être plus active que les autres avocats rencontrés jusqu'ici (voir cas de $\mathrm{M}^{\mathrm{me}}$ Line et de M. Charles): elle essaye de plaider en faveur de son client en mettant en avant son assimilation au pays. Surtout, elle sollicite à plusieurs reprises l'administration afin de clarifier la situation de son client et de permettre à M. Kirin d'obtenir la citoyenneté. 3) Être "contre le droit» ou aller au-delà du droit: déçu par les réponses de l'administration, par la longueur des procédures qui ne lui semble pas justifiée et surtout par la surprise d'un obstacle important, une dette non payée qui remonte à ses études, M. Kirin n'agit plus en accord avec le droit. S'il a mandaté une avocate, il entreprend également des actions sans la consulter. À plusieurs reprises, il prend sa plume et rédige des courriers en manifestant son désaccord et sa déception vis-à-vis de l'action administrative. Il sollicite les responsables des institutions jusqu'à obtenir un rendez-vous.

Malgré les ressources personnelles qu'il déploie (ténacité, sacrifice, persévérance) et ses différentes modalités d'agentivité, $M$. Kirin n'est malheureusement pas gagnant. Toutefois, il ne renonce pas à son désir de se faire naturaliser. Il affirme avoir " un planning" pour "finir cette histoire»: 
Bien sûr. J'ai mon planning. Parce que javais déjà le planning pour finir cette histoire. Mais le chômage m'est tombé dessus. [...] J'avais le planning pour tout finir fin décembre ici. [...] J'avais mes plans. Par exemple, au mois d'avril, j'avais déjà planifié pour tout rattraper en fin décembre. Mais le chômage il tombe à nouveau. Donc le chômage il tombe, étant donné qu'à chaque fois, il m’empêche justement de finir ce retard. Alors que je l'ai déjà payé. Ça m’a créé un trou. Le trou, il est difficile à remonter, hein. Mais ce n'est pas grave. J'essaierai de mon mieux. Si je n'arrive pas, je n'arrive pas. Je m'en fous, hein! Je ne vais pas me forcer hein. J'ai un délai à propos de l'accord de Berne. C'est un délai étant donné, c'est un délai de trois ans. Donc ça allait. Donc depuis 2013, jusqu'en 2016. (M. Kirin, juin 2015)

\subsubsection{UNE AGENTIVITÉ DÉLÉGUÉE ET GAGNANTE:}

\section{LE CAS DE M. FRANÇOIS}

Près de dix années après son arrivée en Suisse en tant que requérant d'asile, M. François dépose une demande de naturalisation avec la motivation suivante: "Je suis en Suisse depuis 10 ans. Je suis diminué dans ma santé, mais la Suisse m'a accueilli. Avec la situation de conflit [dans mon pays d'origine], je pense poursuivre ma vie ici. J'aimerais avoir des droits et des devoirs» (extrait de la demande de naturalisation, octobre 2011).

Il joint de nombreuses attestations à sa demande: quatre attestations de suivi de cours de français, deux attestations de travail, trois attestations de membre d'association, mais aussi quatre lettres de soutien. Ces nombreux documents, en plus des documents demandés lors du dépôt de la demande de naturalisation, démontrent - à notre avis - une certaine agentivité de $\mathrm{M}$. François pour atteindre son objectif. Toutefois, à partir du rapport issu de l'enquête d'intégration menée par le Service de la cohésion multiculturelle (rédigé en janvier 2013), la commune donne un préavis négatif (mai 2013): les connaissances de la langue française sont considérées comme étant insuffisantes. En août 2013, le Service de la justice envoie un courrier à $M$. François pour lui communiquer le préavis négatif. Comment $\mathrm{M}$. François réagit-il à ce refus?

Le cas de M. François illustre spécifiquement un mode d'action possible: mandater un avocat et s'en remettre entièrement à lui. Il lui transmet la lettre reçue par le Service de la justice et, à partir de cet instant, M. François n'intervient plus personnellement dans 
la procédure de naturalisation. Même si son action est réduite, il reste un client qui fait preuve d'agentivité légale.

L'avocat de M. François envoie en septembre 2013 une lettre de trois pages au Service de la justice pour informer que son client maintient sa demande et qu'il «fait part de la présente détermination en exercice de son droit d'être entendu». Cette communication énumère ensuite différents «faits» (terme utilisé par l'avocat), numérotés de 1 à 15, relatifs à la biographie de François. Nous en citons quelques-uns : 1) «il figure parmi les nombreuses victimes de cette guerre sanglante et il est actuellement handicapé»; 2) «malgré sa bonne volonté, il n'a pas pu intégrer le marché de travail»; 3) il «a suivi plusieurs cours de français»; 4) il «a toujours respecté l'ordre juridique suisse»; 5) «son casier judiciaire est vierge»; 6) «il n'a pas de poursuites ni d'actes de défaut de bien»; 7) «il n'est pas connu par les services de la police».

Son niveau de français est communiqué à l'appui de documents. Il est aussi précisé que, "vu la situation (dans son pays), le requérant n'a aucun projet de retourner dans son pays d'origine", et que M. François «est très attaché à la Suisse et est membre de plusieurs associations" (extraits du courrier de l'avocat, septembre 2013). Après la présentation de ces différents "faits", l'avocat mentionne une "jurisprudence constante», ainsi que différents articles de loi relatifs aux critères d'intégration pour les personnes souffrant de handicap. Il se réfere en particulier à une décision du Tribunal fédéral selon laquelle: "Dans le cas de personnes souffrant d'un handicap, les conditions d'intégration doivent être interprétées souplement (ATF 135 I 49)». Il cite aussi l'avant-projet du 16 décembre 2009 de la loi fédérale sur la nationalité suisse (LN), qui dispose dans son article 12 al. 2 relatif au critère d'intégration que «la situation des personnes qui pour des raisons psychiques ne remplissent pas les critères d'intégration prévu à l'al. 1 let. c et $\mathrm{d}$ est prise en compte de manière appropriée». En dépit de ces nombreuses citations, l'avocat précise en fin de courrier qu' «il ne s'agit pas, ici, d'appliquer le projet de loi avant son adoption, mais de prendre en compte la jurisprudence établie du Tribunal fédéral » (extraits du courrier de l'avocat, septembre 2013).

Sur la base de ces arguments, il conclut son courrier en indiquant que son client est «en droit de maintenir sa demande de naturalisation vu qu'il répond à toutes les exigences requises telles qu'elles sont formulées par la loi et précisées par la jurisprudence 
constante du Tribunal fédéral ». L'avocat mentionne aussi les efforts que M. François a fournis malgré les circonstances particulières:

Il a fait tout ce qui est dans son possible pour améliorer son revenu et son niveau de français, étant précisé qu'il n'a fait que l'équivalent de l'école obligatoire en [pays d'origine], son niveau intellectuel, sa situation psychique, son âge et son état de santé ne lui permettent pas d'améliorer davantage sa situation et son intégration. (Extraits du courrier de l'avocat, septembre 2013)

En septembre 2013, le Service de la justice répond à l'avocat que le handicap de M. François a bien été noté, mais que des informations supplémentaires sur sa maladie sont nécessaires avant de «se prononcer définitivement sur la demande de [M. François]». Un certificat médical «expliquant plus précisément sa maladie» est requis. Très rapidement, le 4 novembre 2013, M. François consulte un médecin. Puis, sur demande de celui-ci, il est examiné une nouvelle fois par un confrère spécialisé en neurologie. M. François est guidé par son avocat, dont il suit les recommandations. L'enchaînement entre ces différentes étapes est rapide et efficace. À la suite de ces deux consultations, l'avocat envoie au Service de la justice les deux rapports des deux médecins et indique dans son courrier que «le handicap décrit explique les difficultés de langage et d'apprentissage de (son) mandant» (extrait du courrier de l'avocat adressé au Service de la justice, décembre 2013). Moins d'une semaine après l'envoi du courrier de l'avocat, le Service de la justice informe l'avocat que, désormais, les "conditions pour l'obtention de la naturalisation sont remplies» (extrait du courrier du Service de la justice, décembre 2013) et que la demande de naturalisation est transmise au Secrétariat d'État aux migrations en vue de l'autorisation fédérale de naturalisation.

En mars 2014, M. François obtient l'autorisation fédérale de se faire naturaliser dans le canton, en vertu de l'art. 13 de la loi sur la nationalité de 1990. L'avocat fait alors parvenir au Service de la justice la demande de naturalisation neuchâteloise remplie par M. François. L'avocat joue toujours son rôle d'intermédiaire. M. François ne correspond pas directement avec les services cantonaux et municipaux.

La naturalisation communale est accordée en août 2014. M. François est informé de l'obtention de la naturalisation 
neuchâteloise par un courrier du Service de la justice daté du mois de février 2015, signé par un Conseiller d'État. L'acte de naturalisation est joint au courrier, ainsi qu'un exemplaire de la Constitution de la République et Canton de Neuchâtel.

Dès le début de la procédure, M. François est avec le droit. Toutefois, il trouve nécessaire de faire appel à un avocat pour entreprendre les démarches et obtenir la citoyenneté. Son choix est efficace, son avocat aussi. Grâce à une jurisprudence qui permet d'encadrer clairement le cas de M. François, l'avocat trouve immédiatement des moyens pertinents pour convaincre les autorités à accorder la citoyenneté à son client. Le rapport de M. François avec le droit et le système est bien défini: selon lui, les lois sont claires et ses droits sont respectés.

\subsubsection{UNE AGENTIVITÉ IMPLICITE ET PLEINE: LE CAS DE M. JOHAN}

La dernière histoire présentée, celle de M. Johan, est choisie pour illustrer le cas de figure le plus courant, lorsque la procédure de naturalisation se déroule sans accroc d'un point de vue légal (mais, voir chapitre 6, pour un possible contretemps).

M. Johan est né en Suisse, de parents ressortissants de l'Union européenne. En mars 2013, M. Johan dépose une demande d'autorisation fédérale de naturalisation pour sa fille et lui-même. Il motive sa demande dans le dossier en écrivant: "Je suis né à Neuchâtel il y a trente-sept ans, mes amis et ma famille sont ici, je suis l'heureux père d'une petite fille de 3 ans et je voudrais la voir grandir en tant que citoyenne suisse» (extrait de la demande de naturalisation, mars 2013).

En avril, M. Johan reçoit une lettre du Service de la justice indiquant l'ouverture de son dossier. En novembre 2013, ce même service demande au Service de la cohésion multiculturelle de procéder à une "enquête de naturalisation" au sujet de M. Johan et de sa fille. Le rapport de l'enquête est établi en février 2014. Il se nomme "rapport de naturalisation». Il est indiqué dans le rapport qu'il n'y a pas eu d'entretien, car il s'agit d'une "personne de deuxième génération (procédure simplifiée)». Les transcriptions des entretiens téléphoniques avec deux des trois personnes de référence citées par $\mathrm{M}$. Johan montrent sa très bonne intégration (voir chapitre 6). En voici des extraits: "Je pense qu'être plus intégré que lui, ce n'est pas possible», "comme [M. Johan] est né en Suisse, il n’a pas dû s'intégrer comme un étranger et est totalement intégré 
depuis sa naissance. C'est un Suisse avec des papiers [d'un autre pays]» (extraits du rapport d'intégration, février 2014). Dans le rapport du Service de la cohésion multiculturelle, la case «intégration consolidée» est cochée. Dans le même mois, le Service de la justice écrit au Conseil communal pour demander un préavis sur la base du rapport établi par le Service de la cohésion multiculturelle. En mars 2014, le Conseil communal «préavise» que M. Johan et sa fille "pourront être déclarés ressortissants de la commune de [...], en conformité de la loi sur le droit de cité neuchâtelois» (extrait du courrier du Conseil communal, mars 2014). En juillet 2014, le Secrétariat d'État aux migrations accorde l'autorisation fédérale. En décembre 2014, la naturalisation communale est accordée à M. Johan et sa fille. Ils «sont déclarés ressortissants de la commune de $[\ldots]$, en conformité de la loi sur le droit de cité neuchâtelois» (extrait de la décision du Conseil communal, décembre 2014). En février 2015, un courrier du Service de la justice, signé par un Conseiller d'État, informe M. Johan que la naturalisation neuchâteloise lui est accordée, ainsi qu'à sa fille. Les deux actes de naturalisation sont joints au courrier, ainsi qu'un exemplaire de la Constitution de la République et Canton de Neuchâtel. En mars 2015, le Service de la justice écrit à l'Office de l'état civil pour demander d'enregistrer la décision de naturalisation. À la différence d'autres requérantes ou requérants, $M$. Johan n'a pas entrepris de démarches spécifiques pour répondre aux exigences des autorités. Il explique la motivation de sa demande lors de l'entretien de recherche (juin 2015), en évoquant une raison d'ordre pratique: "Je me suis lancé dans cette naturalisation parce que, ben, j’ai eu une petite fille surtout, surtout ça. Elle est née ici donc euh, je voulais, faire d'une pierre deux coups. [...] C'est surtout pour ça que je me suis fait Suisse. »

M. Johan n'est pas inquiet par rapport à ces démarches. Selon ses propos, c'est lui qui décide de sa naturalisation. Il se positionne comme acteur à plein titre, il n'est pas passif, il se «lance dans cette naturalisation" et se "fait Suisse» (extraits de l'entretien de recherche, juin 2015). Pour lui, la naturalisation est plus une démarche qu'une procédure. C'est assez simple, même si cela prend du temps. Il s'agit d'une formalité qui se résume à récupérer des papiers, à les remplir et à attendre. M. Johan ne peut pas énoncer les étapes qui se sont déroulées entre le moment où il a déposé sa demande et la réception de son passeport. Il est très hésitant à ce 
sujet lors de l'entretien de recherche (juin 2015) et fait part de son peu d'intérêt pour la question:

- Chercheuse: La manière dont s'est déroulée la procédure? Est-ce qu'il y a quelque chose qui vous a... frappé? Comment vous avez trouvé les informations par exemple?

- M. Johan: Ben les informations, claires... Comme ça s'est déroulé. Non j'ai juste été chercher les papiers à... c'est quoi l'Office des naturalisations, là?

- Chercheuse: Service de justice, oui. Où y a l'état civil aussi.

- M. Johan: Exactement. Voilà! Et là, ils mont donné les papiers, j’ai rempli ces papiers, y a pas eu trop de, ce n'est pas trop compliqué à les remplir, et puis hein, et puis j'ai envoyé ça et puis c'est vrai que ça a, que ça a pris quand même pas mal de temps, avant de, de recevoir les réponses, mais...

Finalement, pour M. Johan, obtenir le passeport suisse ne provoque pas de changement identitaire, mais facilite sa vie au quotidien. Il évoque à nouveau des éléments d'ordre pratique:

- Chercheuse: Et vous vous sentez différemment maintenant avec le passeport suisse? Ça change quelque chose, vous avez l'impression?

- M. Johan: Peut-être pour les, quand on passe les douanes, oui. Oui là, peut-être, à l'aéroport ou, c'est plus, plus fluide, on passe moins de temps, voilà.

- Chercheuse: Par exemple pour revenir en Suisse après un voyage ou comme ça?

- M. Johan: Exactement. Sinon, sinon, non, ça n’a rien changé.

\subsection{SYNTHÈSE: DEVENIR ACTRICE ET ACTEUR \\ DE SON HISTOIRE LÉGALE}

Notre sixième hypothèse nous invitait à considérer l'agentivité de la personne candidate à la naturalisation (1.4.3), contrecarrant de ce fait une vision passive de l'étranger comme quelqu'un à assimiler, à accoutumer et/ou à intégrer - vision qui est souvent véhiculée dans les textes de loi et dans les messages du législateur. Ce chapitre a reconstruit les trajectoires administratives et légales de cinq personnes candidates à la naturalisation et a montré la manière dont 
elles se sont engagées activement tout au long de la procédure. Les analyses, réalisées à partir du cadre théorique et méthodologique proposé dans la première partie de ce chapitre, ont ainsi permis non seulement de donner la parole à ces personnes rencontrées lors des entretiens de recherche, mais ont aussi rendu visible leur capacité à devenir actrices et acteurs de leur histoire légale.

Nous présentons ici quelques réflexions conclusives autour des parcours que nous venons d'analyser: ceux de $\mathrm{M}^{\text {me }}$ Line, de MM. Charles, Kirin et François, qui sont particulièrement complexes et présentés comme problématiques par le Service de la justice, et celui de M. Johan, qui suit une procédure simplifiée au sens du droit cantonal (chapitre 5).

Nous allons construire cette synthèse autour de quelques questions clés: comment ces personnes se positionnent-elles vis-à-vis de la procédure? Comment réagissent-elles face à des obstacles? Comment leur agentivité se manifeste-t-elle? Enfin, quels sont les facteurs qui empêchent la plupart de ces personnes d'obtenir la nationalité?

Pour répondre à la première de nos questions, et ce en retraçant le lien entre les deux catégories sociologiques guidant notre lecture - la conscience juridique et l'agentivité légale -, nous avons constaté qu'au début de la procédure, ces personnes sont «avec le droit", voire "face au droit» : elles se sentent en accord avec la loi et légitimes à demander la nationalité. Toutefois, les réponses qu'elles obtiennent de la part des institutions et les obstacles rencontrés, l'expérience qu'elles font du droit et de la démarche administrativo-légale, provoquent un changement dans leur posture. C'est ainsi que si $\mathrm{M}^{\mathrm{me}}$ Line, $\mathrm{MM}$. Kirin et François continuent leur combat et essayent de faire avec la loi, $\mathrm{M}$. Charles fait part de sa grande déception et de sa colère vis-à-vis des obstacles rencontrés et renonce à se battre. Dans le récit de $\mathrm{M}$. Johan, cette expérience, qui ne renvoie à aucune difficulté, tend à être minimisée, voire à devenir invisible.

Les obstacles principaux auxquels ont été confrontés les protagonistes sont liés à un ensemble de facteurs: personnels, comme dans le cas de M. François, ou contextuels, comme la crise économique qui empêche MM. Kirin et Charles de trouver un travail, de régler leurs dettes et de répondre ainsi aux exigences de la loi. $\mathrm{M}^{\mathrm{me}}$ Line est confrontée à une complication similaire lorsque sa famille rencontre des difficultés financières et que les dettes de son mari 
entravent sa demande de naturalisation. La question qui semble se poser dans tous les cas - à part dans celui de M. Johan, qui n'est confronté à aucun problème - est: comment réagir et surmonter ces obstacles afin d'obtenir la citoyenneté?

Dans les cas analysés, un des premiers constats que nous pouvons faire est que les personnes confrontées à ces obstacles ressentent tout d'abord de l'incompréhension: M. Charles raconte qu'une semaine après le dépôt de sa demande, il a été informé de l'existence d'une dette qu'il ignorait jusqu'alors; M. Kirin découvre qu'il doit payer une dette qu'il a contractée lors de ses études; quant à $\mathrm{M}^{\mathrm{me}}$ Line, elle fait face à la dette de son mari, qui doit être réglée pour qu'elle puisse obtenir la naturalisation. Que font alors les candidates et les candidates face à ces surprises?

Tout d'abord, ces personnes essayent de comprendre la raison du refus et/ou de la suspension de la procédure de naturalisation: elles se renseignent, posent des questions à l'administration. Ensuite, elles cherchent à mettre en place des solutions, seules ou avec l'aide d'experts, par exemple une avocate ou un avocat, ou encore avec l'aide d'amies ou amis. Si M. Charles agit de façon plutôt individuelle, $\mathrm{M}^{\mathrm{me}}$ Line et $\mathrm{MM}$. Kirin et François optent pour des stratégies différentes, parfois en combinant plusieurs modes d'action, faisant notamment appel à d'autres personnes ou dialoguant directement avec les responsables au sein des administrations. Il s'agit de formes d'agentivité oscillant entre une dimension individuelle et une dimension socialement partagée, au sens du cadre théorique développé en amont.

Malgré le degré d'agentivité plus ou moins élevé de chaque candidat, les résultats ne sont pas toujours positifs: seuls $\mathrm{M}^{\mathrm{me}}$ Line et $\mathrm{M}$. François obtiennent la citoyenneté, $\mathrm{M}^{\mathrm{me}}$ Line près de vingt ans après le début de la procédure et $M$. François grâce à une jurisprudence précise qui peut être appliquée à son cas. Qu'en est-il de MM. Charles et Kirin? Les deux candidats se sont en quelque sorte égarés dans la procédure pour une raison similaire, même s'ils ont des réactions différentes: ils passent par des périodes de chômage et ne sont ainsi pas en mesure de remplir le critère de l'autonomie financière attendu par la loi. Toutefois, si M. Kirin est prêt à prendre les devants avec son "planning", en cherchant un travail, en réglant ses impôts, $M$. Charles, lui, résiste à l'idée de devoir "se conformer" à l'administration. Il ne partage pas la même vision de l'intégration, comme il a pu en témoigner lors de 
l'entretien de recherche: "ça ne joue pas avec la loi», "ça ne me plaît pas comme ça» (voir chapitre 6). Ce ne sont plus les autorités qui refusent de reconnaître son intégration, c'est son choix. Par ce discours, M. Charles se positionne comme étant maître de la décision et de son parcours de naturalisation à part entière.

Comment l'agentivité se manifeste-t-elle? Comme nous avons pu le constater, dans les différents cas examinés, les protagonistes s'approprient peu à peu les règles du système tout en jouant avec (cas de $\mathrm{M}^{\mathrm{me}}$ Line, de MM. Kirin et François) ou encore en se démarquant de celles-ci, par exemple en produisant un discours contestataire (cas de M. Charles). Leur agentivité évolue au fur et à mesure de la procédure, dans la façon de répondre aux administrations, de rédiger leurs courriers et de prendre des initiatives. L'acquisition et l'évolution de leurs compétences sont spécialement visibles lors de l'analyse des courriers qu'ils rédigent à l'attention des administrations et de la justice. Dans un premier temps, ces courriers sont empreints d'émotions et sont personnalisés. Certains courriers sont rédigés à la main. Les histoires subjectives sont mises en avant, les faits sont traduits avec des termes émotionnels. Ce type de langage évolue dès que l'histoire est canalisée dans un parcours juridique et est transformée conformément aux exigences de la procédure, sans forcément supprimer l'expression de valeurs ou d'émotions et plus généralement de leur identité.

Cette évolution dans la rédaction des courriers témoigne de l'appropriation progressive de règles explicites et implicites. Toutefois, ces courriers, mêlant des arguments de la vie quotidienne et des données factuelles, visant à justifier le plus souvent une situation professionnelle ou financière, n'ont pas automatiquement un impact sur le déroulement de la procédure. Seulement dans un cas, celui de $\mathrm{M}^{\mathrm{me}}$ Line, nous avons constaté que son agentivité fondée à la fois sur des arguments juridiques pertinents et sur des arguments culturels et émotionnels - spécialement dans le cadre du recours au Tribunal fédéral - a été gagnante. Elle a aussi eu un impact plus général sur la procédure de naturalisation, spécifiquement dans le cadre de la construction du dossier de l'administration.

Comment expliquer alors la difficulté de ces clientes et clients pour obtenir la nationalité? Quels sont les facteurs qui les empêchent d'obtenir la nationalité?

La difficulté d'aboutir à la fin de la procédure est peut-être attribuable à plusieurs facteurs, dont certains peuvent être qualifiés de 
contextuels ou d'objectifs et d'autres de personnels ou de subjectifs. Parmi les facteurs du premier type, un élément non négligeable est le fait que la procédure de naturalisation est peu intelligible (chapitres 5 et 6). En outre, la naturalisation n'est pas un droit, ce qui a pour effet de donner une marge de manœuvre à l'administration. En effet, si dans le cas de M. François, l'avocat obtient satisfaction grâce à une jurisprudence bien établie, dans le cas de M. Charles, il n'y a pas de violation apparente à évoquer. Le parcours légal de $\mathrm{M}^{\mathrm{me}}$ Line est plus complexe. L'existence de lacunes évidentes dans l'établissement des faits lui donne la possibilité d'agir au niveau de la justice et de faire appel d'abord au Tribunal cantonal et ensuite au Tribunal fédéral, en invoquant la violation de plusieurs droits constitutionnels. La difficulté à s'opposer à la marge d'appréciation des autorités, et par conséquent de recourir aux garanties procédurales, peut aussi expliquer les limites du travail des avocats, qui, par exemple dans les cas de MM. Charles et Kirin (et même de $\mathrm{M}^{\mathrm{me}}$ Line), ne peuvent pas faire grand-chose, si ce n'est demander un dossier à l'administration et rassembler des documents. Les résultats de leur action sont ainsi peu satisfaisants aux yeux de MM. Charles, Paul et Kirin et de $\mathrm{M}^{\mathrm{me}}$ Line, même M. Kirin, qui est assisté par un avocat plus efficace que ceux de $\mathrm{M}^{\mathrm{me}}$ Line et M. Charles, décide d'agir de façon autonome. Aux facteurs mentionnés, nous pouvons ajouter une ligne rigide de l'administration neuchâteloise, qui à l'époque exigeait que la totalité des impôts soit réglée par le candidat. Cette ligne rigide semble avoir été assouplie au fil du temps: le cas de $\mathrm{M}^{\text {me }}$ Line, par exemple, arrive à conclusion grâce, apparemment, à la décision finale des autorités neuchâteloises de distinguer ses obligations de celles de son mari.

Parmi les facteurs du premier type, on peut enfin mentionner les conditions économiques, qui empêchent fréquemment les personnes concernées (cas de MM. Charles et Kirin) de trouver un emploi et de pouvoir subvenir à leurs besoins, et, le cas échéant, de régler leurs dettes, notamment leurs impôts, en répondant ainsi aux exigences de la loi ou, du moins, de la pratique interprétative de celle-ci.

Les obstacles du deuxième type sont plutôt liés à la spécificité du ou des parcours de vie de certaines personnes: par exemple, la difficulté d'apprendre la langue locale à cause d'un handicap, à la suite de blessures de guerre dans le pays d'origine (cas de M. François); la difficulté d'accéder à un travail qualifié en raison de sa trajectoire 
(cas de M. Charles) ; la volonté de garder ses coutumes, par exemple vestimentaires, vis-à-vis de la Commission des naturalisations (cas de $\mathrm{M}^{\mathrm{me}}$ Line) ; mais aussi la difficulté de comprendre et d'interpréter la loi et la pratique de l'administration quant aux attentes en matière d'intégration (tous les cas).

Pour conclure, malgré les efforts de ces clientes et clients pour parvenir à des résultats, la procédure de naturalisation semble s'apparenter à une course d'obstacles, qui les empêche de développer une agentivité légale gagnante (à part dans les cas de $\mathrm{M}^{\mathrm{me}}$ Line et M. François) ${ }^{35}$, en raison de la combinaison des facteurs personnels ou subjectifs et contextuels ou objectifs que nous venons d'évoquer.

35. Centlivres, 1991. 



\title{
8. RENDRE DES DÉCISIONS: TRAJECTOIRE DES DOSSIERS
}

\author{
ANNE LAVANCHY ET ÉLODIE GARROS
}

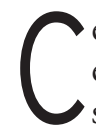

e chapitre analyse l'intégration du point de vue des institutions, en se centrant sur le travail administratif et les processus décisionnels. Il s'intéresse aux processus d'écriture et d'évaluation à partir des questions suivantes: comment les critères sur lesquels repose l'évaluation de l'intégration sont-ils rendus opérationnels, au quotidien, par les actrices et acteurs administratifs? Comment sont sélectionnés les éléments pertinents, aux yeux des institutions, pour évaluer l'intégration? Comment la circulation des informations est-elle organisée entre les divers services administratifs, et entre les instances cantonales, communales et fédérale? Comment les décisions d'octroi du droit de cité ou de refus sont-elles prises?

À travers ces questions, il s'agit de dégager les conditions nécessaires au déploiement de l'autorité institutionnelle, le pouvoir respectif des actrices et acteurs impliqués, leur marge d'appréciation et leur capacité d'influencer le parcours d'une demande ${ }^{1}$. Plus précisément, ce chapitre discute trois des sept hypothèses exploratoires formulées au chapitre 1. Il s'agit de comprendre les modalités et significations des démarches effectuées pour vérifier, et quantifier, l'intégration d'une personne, et les prises de décision que ces modalités entraînent (deuxième hypothèse); d'examiner le travail de l'administration en tant qu'entreprise de production de sens et de légitimité, qui se traduit notamment par la production de traces lexicales et matérielles dont les effets sont performatifs (troisième hypothèse); et d'analyser les dynamiques spécifiques de la trajectoire administrative (septième hypothèse).

Si la procédure de naturalisation est appréhendée dans son ensemble, ce chapitre discute en particulier la question de 
l'intégration. Ce n'est pas le seul critère de naturalisation, mais c'est celui qui demande le travail le plus approfondi du côté des services administratifs. En effet, il n'est pas défini ni déterminable de manière univoque. En tant que critère réputé matériel, pour reprendre la distinction faite dans la loi sur la naturalisation en vigueur dès le $1^{\text {er }}$ janvier 2018, son évaluation comporte une marge d'appréciation beaucoup plus étendue que celle s'appliquant aux critères formels tels que, par exemple, le nombre d'années de résidence en Suisse.

Après une présentation du corpus analysé et de l'ancrage théorique (8.1), le chapitre distingue deux dimensions. En premier lieu, le traitement administratif est une procédure de papier(s); les pratiques professionnelles s'incarnent dans des choses concrètes, dans des artefacts, dans des documents, regroupés dans un dossier nommé «dossier administratif» (8.2). Mais comment ce dossier est-il constitué? Quelles sont les contraintes induites par des trames spécifiques, et particulièrement celle du formulaire qui constitue le rapport d'intégration? Il s'agit de comprendre ce qu'est un dossier et comment il est façonné, puis d'analyser en quoi les documents qui le forment sont à la fois des traces, des outils et des résultats. En second lieu, il s'agit d'analyser la manière dont les décisions sont rendues - et, donc, comment se clôt un dossier (8.3). Quels sont les processus décisionnels qui permettent la clôture du dossier? Et quelles sont les marges de manœuvre et pouvoirs respectifs des différents agentes et agents de l'État qui permettent de faire pencher la balance de l'un ou l'autre côté? Pour répondre à ces questions, ce chapitre aborde la thématique du pouvoir décisionnel, puis la manière dont les représentations liées à la réputation du canton influencent les discours sur la pratique de l'octroi du droit de cité, pour s'achever par l'analyse des dynamiques d'influence et de consensus.

\subsection{ANALYSER LA PROCÉDURE DE NATURALISATION}

Étudier de manière compréhensive les pratiques professionnelles de la naturalisation nécessite un traitement de données multiples, produites lors des différentes opérations de recherche: la transcription des entretiens formels, enregistrés; les notes consignant les entretiens informels et discussions d'explicitation, non enregistrés; les notes sur les observations effectuées dans les services cantonaux et communaux concernés. Par ailleurs, les cinq dossiers complets, reconstitués et réagencés à plusieurs reprises, fournissent 
des informations essentielles sur le traitement administratif, car ils rendent explicite la manière dont les informations sont synthétisées et reformulées, notamment lorsqu'il s'agit de les faire circuler entre les différents services cantonaux et entre les administrations cantonale, fédérale et communale.

Les analyses spécifiques développées dans ce chapitre mobilisent les recherches menées sur les institutions formelles ${ }^{2}$, qui ont connu un regain d'intérêt depuis le début des années 2000. Si ces dernières sont souvent centrées sur la rencontre aux guichets ${ }^{3}$, ce corpus explore la vie sociale des lois ${ }^{4}$, la manière dont elles sont mises en application ${ }^{5}$, les prises de décision institutionnelles, ainsi que le rôle du papier comme technique administrative fiable, indispensable à la production d'entités étatiques lisibles ${ }^{6}$. Il permet aussi d'interroger les caractéristiques tacites, tenues pour acquises, de la vie organisationnelle, et de documenter les prises de décision, les types de pouvoir et la manière dont ces pouvoirs sont mis en mots ou, au contraire, tus ${ }^{7}$.

Les données utilisées dans ce chapitre ont été produites, d'abord, lors d'un travail de terrain dans les deux principaux services cantonaux impliqués à Neuchâtel: le secteur naturalisation du Service de la justice et le Service de la cohésion multiculturelle. Elles incluent un entretien mené avec le Service des migrations, ainsi que des informations récoltées auprès des communes. Dans une perspective psychosociale ${ }^{8}$, recueillir les discours à propos des pratiques ne se limite pas à un intérêt méthodologique: en effet, les personnes produisent des représentations en fonction de leurs propres expériences et pratiques, mais aussi selon les cadres de pensée définis par le contexte socioculturel dans lequel elles évoluent ${ }^{9}$. Le terrain dans les administrations cantonales a considéré différents niveaux hiérarchiques: le personnel subordonné, les responsables intermédiaires et les cheffes et chefs de service. Il s'agit d'un corps professionnel hétérogène qui, comme celui d'autres agentes et agents de l'État, se caractérise par le fait qu'il ne « [maîtrise] jamais l'ensemble

2. Crozier et Friedberg, 1980; Merton, 1949; Weller, 2009; Weller, 2013.

3. Dubois, 1999; Eule, 2014; Spire, 2008.

4. Eckert, 2008; Nader, 1965.

5. Kelly, 2008; Latour, 2002; Lavanchy, 2014

6. Hull, 2012; Mathur, 2012; Scott, 1998.

7. Castellano, 2007; Laurens, 2008.

8. Appadurai, 1986; Graeber, 2012; Henare, Holbraad et Wastell, 2006; Hull, 2012; Kalampalikis et Apostolidis, 2016. Latour, 1994; Moscovici, 1984

9. Jodelet, 2006, 2008 et 2015. 
$\mathrm{du}$ processus de fabrication du service auquel [ses membres] concourent, [ces derniers ne revendiquant pas une appartenance] à un métier qui leur serait spécifique» ${ }^{10}$. Occupant des positionnements distincts au sein de l'administration, les membres de ce corps professionnel ont des marges d'action différenciées et sont soumis à des impératifs et autorités variables. Ainsi, si les cheffes et chefs de service n'ont pas d'action directe sur la trajectoire des dossiers d'un point de vue technique, ils jouent un rôle important dans l'organisation du travail à l'intérieur du service. Ils sont aussi chargés de coordonner les impératifs administratifs et politiques, puisqu'ils sont directement subordonnés à la personne, élue, responsable du dicastère.

Pour appréhender la complexité des processus décisionnels, nous avons ensuite inclus les communes qui, pour les procédures antérieures à 2018, interviennent au cours des deux phases de la procédure, celle de l'autorisation fédérale et celle de l'octroi du droit de cité. C'est l'ensemble de ces collaboratrices et collaborateurs qui sont identifiés comme formant le corps professionnel de la naturalisation, et nommés ici indifféremment actrices et acteurs de l'administration, personnel administratif ou agents de l'État.

Lors du travail de terrain, il n'a pas été possible d'assister à des rencontres au guichet, les dossiers analysés étant clos (voir chapitre 2). De plus, ce type de rencontre est rare dans les espaces dans lesquels la recherche a été menée: il n'y a pas de visite spontanée durant les heures d'ouverture des services, mais des entretiens sur convocation avec le Service de la cohésion multiculturelle et la commune. Notre intérêt s'est plutôt porté sur la possibilité de "suivre le cours d'une décision publique, depuis la lente et patiente fabrication des dossiers ${ }^{11}$. Il s'agissait d'observer le travail administratif en train de se faire, la manière dont certaines feuilles de papier deviennent des documents, et comment ces derniers sont agencés pour former les dossiers, ces ensembles à la fois matériels et symboliques ${ }^{12}$ qui produisent des identités administratives. Ces observations ont isolé, dans le travail ordinaire à l'intérieur des services, certains types d'activités en lien avec la fabrique de l'intégration et ne prétendent pas couvrir l'ensemble du quotidien professionnel de leur personnel.

10. Weller, 2009, p. 322.

11. Ibid., p. 326.

12. Torpey, 1997. 
Il convient aussi de souligner la diversité des compréhensions de ce qu'est l'intégration: le Service de la cohésion multiculturelle et le Service de la justice ont des regards contrastés. Le premier estime que l'intégration est un processus constant qu'il n'est pas possible de mesurer comme s'il s'agissait d'un état ("être intégré»). Pour des raisons historiques présentées ci-après, le service s'est battu pour inclure la conception dynamique qu'il défend dans la procédure: "L'intégration, ce n'est pas un point X, c'est un processus", dit M. Corentin (avril 2015). Au sein de chacun des services, les positionnements individuels face à ce que devrait être une bonne et satisfaisante intégration, et, partant, comment doivent être définies les futures concitoyennes et concitoyens, peuvent aussi varier. La question de la désirabilité ${ }^{13}$ est centrale dans le système de naturalisation helvétique, où obtenir la nationalité n’est pas un droit automatique en dehors du principe de jus sanguinis. La Suisse étant définie comme une Willensnation ${ }^{14}$, requérantes et requérants doivent vouloir faire - et devenir - comme "nous, les (vrais) Suisses", un "nous" collectif qui s'affirme en définissant ses frontières et les manières de les traverser ${ }^{15}$. Leur désirabilité est jugée à l'aune de cette volonté, et est nécessaire pour que les agents de l'État leur ouvrent les portes de l'appartenance nationale.

Si la procédure de naturalisation implique les trois niveaux politiques et administratifs suisses - la Confédération, le canton et la commune -, le niveau cantonal est prépondérant à Neuchâtel. C'est le Service (cantonal) de la justice qui est responsable de la procédure. Il monte le dossier, collecte les renseignements sous forme de documents, identifie les potentielles informations manquantes et agence l'ensemble de ces éléments pour évaluer la recevabilité de la demande. C'est lui qui coordonne la circulation des informations avec les autres instances administratives et politiques.

\subsection{UNE PROCÉDURE DE PAPIER(S)}

La prévalence de l'écrit caractérise l'organisation du travail administratif. Celui-ci s'incarne dans les documents et artefacts bureaucratiques qui sont les outils permettant de transmettre les informations et de communiquer entre les services. Ce faisant, ils

13. Agier 2008; Fortier, 2013; Lavanchy, 2015.

14. Wimmer, 2011; Zimmer, 2011.

15. Barth, 1969. 
contribuent à forger la légitimité administrative propre à la logique étatique ${ }^{16}$. Les documents objectivent la demande de naturalisation et véhiculent du contenu, des mots, des images, des graphiques, des idées, du pouvoir ${ }^{17}$. Ils constituent à la fois les traces et les objets de l'activité administrative.

\subsubsection{CE QU'EST UN DOSSIER}

Au-delà de leur contenu, les documents de la procédure de naturalisation sont des objets de l'activité administrative dont l'analyse permet de rendre visibles et de questionner les pratiques professionnelles ainsi que ce que produit l'écriture ${ }^{18}$. L'attention portée à la dimension matérielle rend compte de l'association entre humains et non-humains - les objets par exemple - qui se définissent mutuellement dans l'action ${ }^{19}$. Les objets servent d'indices et de repères qui structurent les actions: ce sont non seulement des instruments des organisations, mais aussi des médiateurs et des béquilles pour les processus cognitifs ${ }^{20}$. Ils attestent en quelque sorte d'une "périphérisation de la vie psychique» ${ }^{21}$ ou encore cristallisent sous forme de signes des expériences humaines et des significations élaborées socialement ${ }^{22}$. Ainsi, dans les pays de droit écrit, la fabrique de ce dernier "a pour enveloppe corporelle une chemise cartonnée liée par des élastiques" ${ }^{23}$.

Dans la lignée des travaux qui reconnaissent l'agentivité des non-humains ${ }^{24}$, des auteurs emploient les termes d'agentivité textuelle ${ }^{25}$ pour souligner que les documents ont une capacité d'agir et de faire agir. Toutefois, l'agentivité d'un texte repose sur son inscription dans un ensemble plus vaste. Elle dépend d'autres textes (métatextualité), mais aussi des conversations qui y font référence ${ }^{26}$. Un document, un objet, est toujours le fruit d'une relation, d'une rencontre avec des personnes ${ }^{27}$. Ainsi, l'activité

16. Torpey, 1997; Scott, 1998.

17. Allard, 2012; Bacigalupo, 2016.

18. Borzeix et Fraenkel, 2001; Fraenkel, Pontille, Collard et Deharo, 2010; Gardey, 2008; Jacobsson, 2016; Prior, 2016.

19. Barbier et Trépos, 2007; Latour, 1994.

20. Hull, 2012; Norman, 1993; Ogien et Quéré, 2005; Vinck, 2009.

21. Blandin, 2002, p. 180.

22. Gillespie et Zittoun, 2010.

23. Latour, 2002, p. 83.

24. Callon, 2006; Callon et Law, 1997; Houdart et Thiéry, 2001; Latour, 1994; Latour, 1996.

25. Cooren, 2004.

26. Ibid.

27. Blandin, 2002. 
scripturale joue un rôle important dans la coopération entre divers services par la transmission d'informations ${ }^{28}$. A Neuchâtel, la procédure de naturalisation est ouverte lorsque le Service de la justice reçoit le formulaire "Demande d'autorisation fédérale de naturalisation" dûment complété et assorti de plusieurs pièces administratives à fournir. Ce document témoigne de la volonté de demander la naturalisation. Il opère un changement dans le statut de la personne, qui devient, dans la terminologie du Service de la justice, requérante, et déclenche techniquement la procédure. Dès sa réception, le personnel administratif commence à mobiliser et à produire de nombreux documents qui prennent des formes variées:

- les formulaires, qui sont des documents préétablis devant être complétés par les personnes candidates, comme le sont la «demande d'autorisation fédérale de naturalisation» ou le "formulaire de demande de naturalisation neuchâteloise»;

- les courriers postaux ou électroniques assurant la communication entre les différents services. Ils sont en général produits par ou à l'intention du Service de la justice et impliquent, selon les cas, le Service de la cohésion multiculturelle ou la police cantonale, le Conseil communal, le Secrétariat d'État aux migrations, du côté des institutions, et, du côté des personnes candidates, ces dernières, leurs avocates ou avocats, ou d'autres personnes de confiance susceptibles de servir de témoins lors de la procédure;

- les pièces justificatives fournies par les requérantes et requérants. Elles attestent de leur identité et de leur moralité administrative (attestation d'emploi et de résidence, copie des papiers d'identité, copie des permis de séjour, $\mathrm{CV}$, lettres de recommandation, extrait du registre des poursuites, attestation du Service des contributions, extrait d'antécédents judiciaires);

- les procès-verbaux des organes décisionnels, comme ceux émis par la Commission communale des naturalisations et des agrégations et par la commission chargée par le Conseil d'État de l'examen préalable des dossiers;

- les actes officiels autorisant à passer à la suite de la procédure ou octroyant le droit de cité (autorisation fédérale de naturalisation, octroi du droit de cité communal et octroi du droit de cité cantonal) ;

28. Denis, 2011; Grosjean et Lacoste, 1998. 
- les rapports réalisés respectivement par la police et par le Service de la cohésion multiculturelle suivant la date de la procédure («rapports d'intégration" ou "rapports d'enquête»);

- les préavis cantonaux et communaux.

Ces catégories forment une typologie de base, qui n'est pas exhaustive dans la mesure où d'autres éléments peuvent être requis en fonction de la situation personnelle et de la trajectoire des candidates et candidats. Par exemple, dans le cas de M. François, des expertises médicales ont été ajoutées au dossier, et celui de $\mathrm{M}^{\mathrm{me}}$ Line inclut des jugements de tribunaux (chapitre 7).

Les documents produits et circulant lors de la procédure ont plusieurs fonctions: certains, par exemple une attestation de domicile, servent à informer et certifier; d'autres à contraindre, à transmettre, à prouver; et, dans le cas d'actes performatifs, à agir et façonner le monde social, comme l'octroi du droit de cité. La naturalisation devient donc effective par l'établissement d'un dernier document, performatif: l'acte de naturalisation. Les fonctions peuvent se conjuguer les unes avec les autres. Certains des documents qui s'accumulent au cours de la procédure ont un destinataire spécifique, ce qui est le cas des courriers, alors que d'autres n'en ont pas, ou s'adressent à tout le monde, par exemple une attestation de domicile. Enfin, une partie des documents doit être produite par les personnes requérantes, alors que le reste est généré par la procédure administrative elle-même - c'est par exemple le cas du rapport d'intégration établi par le Service de la cohésion multiculturelle.

Le dossier de naturalisation est formé par l'ajout successif de documents dans une chemise cartonnée de couleur marron. Le personnel du Service de la justice, dans les locaux duquel il est physiquement gardé, l'appelle le dossier brun. La page de garde du dossier, complétée au fur et à mesure de la procédure, est constituée par un document qui renseigne sur l'identité de la personne requérante et sur les dates de deux décisions clés pour l'obtention de la naturalisation, à savoir l'autorisation de naturalisation communale et la décision finale du Conseil d'État. La couverture du dossier offre donc un résumé synoptique de la procédure de naturalisation.

Depuis le mois de juin 2014, la procédure est assistée par le logiciel informatique GESTSTAR. Pendant la phase de terrain, les dossiers avaient simultanément une existence matérielle et 
informatique, dont les formes respectives n'étaient pas totalement identiques. En ce qui concerne la correspondance, le dossier papier ne comprenait plus que les courriers ayant «une importance réelle pour la décision", selon $\mathrm{M}^{\mathrm{me}}$ Juliette (entretien non enregistré, septembre 2016). Sa collègue, $\mathrm{M}^{\mathrm{me}}$ Justine, a aussi expliqué:

Avant, dans le dossier, on avait toutes les copies de nos courriers. Maintenant on ne les a plus qu'informatiquement. Nos courriers, on ne les met plus systématiquement au dossier, sauf s'ils ont une importance réelle pour la décision. Si c'est une explication des conditions qui ne sont pas remplies par exemple, pourquoi on pourrait refuser la naturalisation, ça, on va verser au dossier pour que la commune y ait accès, puis on va aussi le transmettre au niveau fédéral. ( $\mathrm{M}^{\mathrm{me}} \mathrm{Justine,} \mathrm{décembre} \mathrm{2014)}$

La présentation de GESTSTAR met en évidence le travail constant de sélection des informations pertinentes et des documents importants: il s'agit d'anticiper, tout au long du processus, ce qui sera requis pour que la décision puisse être rendue. Cela implique donc de laisser des éléments de côté, mais aussi de mettre en forme les informations retenues pour en permettre le traitement décisionnel.

\subsubsection{TRACES, OUTILS ET RÉSULTATS}

Les documents constituant le dossier administratif sont à la fois les traces, les outils et les résultats de l'activité administrative. Leur analyse implique de considérer la sélection des informations, mais aussi la manière dont ce qui est retenu est traduit dans et par des documents et ensuite lu et interprété dans le cadre de la procédure. À cet égard, les pièces maîtresses sont le rapport de naturalisation et le rapport d'entretien, rédigés sur mandat du Service de la justice par le Service de la cohésion multiculturelle ou, pour les procédures antérieures à 2009, par la police cantonale. Le premier est établi pour toute demande de naturalisation ordinaire. Le second n'est fait qu'en cas d'entretien, et est donc inexistant dans les dossiers des personnes légalement qualifiées de deuxième génération, qui bénéficient d'une procédure simplifiée. Les deux rapports rendent compte de la situation de la requérante ou du requérant à un moment donné. Leur structure et leur dénomination sont décidées par le Service de la justice en collaboration avec le Service de la 
cohésion multiculturelle. Ils constituent par conséquent un outil collaboratif qui circule entre les administrations impliquées dans la procédure de naturalisation. Ce "formulaire élaboré en collaboration" avec le Service de la justice a nécessité plusieurs "tests" avant d'arriver à un "essai concluant» (M. Costin, entretien non enregistré, octobre 2016).

Les agents de l'État ont souvent souligné la distinction entre l'enquête, compétence du Service de la cohésion multiculturelle, et la gestion du dossier, incombant au Service de la justice: "Ce qui arrive parfois, c'est que le Service de la justice reconvoque les gens pour des précisions. C'est eux qui gèrent le dossier. Nous, on fait la partie enquête d'intégration» (M. Corentin, juin 2014).

L'enquête d'intégration se fait par le biais d'un entretien. Présenté comme un espace d'interaction qui permet de "compléter le dossier avec les informations qu'ils transmettent» ( $\mathrm{M}^{\mathrm{me}}$ Corinne, juin 2014), il sert aussi à déterminer, le cas échéant, quels documents supplémentaires sont nécessaires - mais «ce n'est pas une enquête de police, c'est un entretien» (M. Corentin, juin 2014). Les personnes chargées de le mener sont présentées comme étant clairement distinctes de leurs collègues impliqués dans les prestations de consultation et de médiation, l'autre tâche du Service de la cohésion multiculturelle. La raison de pareille "séparation" est de garantir un "travail qui se veut le plus neutre possible» (M. Corentin, juin 2014). Ainsi, dans le cadre de la procédure de naturalisation, la mission du secteur naturalisation du Service de la cohésion multiculturelle est de "donner des informations" $\left(\mathrm{M}^{\mathrm{me}}\right.$ Justine, décembre 2014) à partir desquelles une évaluation de l'intégration pourra être réalisée.

Les entretiens suivent une trame commune dont l'objectif, selon les employées et employés du Service de la cohésion multiculturelle, n'est pas de tester les connaissances à propos de la Suisse. C'est bien plus la vie sociale et associative qui est au centre de la "connaissance» à évaluer, et plus précisément la participation à ce tissu social spécifique: "Est-ce que les gens sont membres d'une association, d'une collectivité étrangère, de notre point de vue, c'est considéré comme une participation à la société [...]. Ce sont des éléments comme ça, [...] ce qui leur plaît [...]. C'est [...] un dialogue avec des questions très ouvertes" (M. Corentin, avril 2015). Plutôt qu'un dialogue, M. Costin parle d'un "questionnaire» qui reprend systématiquement les mêmes questions (septembre 2014) 
et $\mathrm{M}^{\mathrm{me}}$ Corinne mentionne un canevas plus structuré: «En fait, il y a en tout cinq questions" (juin 2014). Les variations de terminologie indiquent, d'une part, que chaque agente, chaque agent de l'État confere à l'entretien une fonction spécifique reflétant sa propre perception de ce qu'est l'intégration et de son rôle dans la procédure de naturalisation et, d'autre part, que le formulaire revêt un caractère évolutif: lors des deux années qu'a duré le travail de terrain, le formulaire a fait l'objet de fréquentes adaptations et changé de forme plusieurs fois. Ces modifications semblent toutes tendre vers une formalisation grandissante, précisant les canevas et les unifiant.

Le formulaire a un effet sur le contenu et l'évaluation de l'intégration des personnes requérantes. La forme matérielle d'un rapport d'intégration guide et transforme les informations sélectionnées ${ }^{29}$. Comme la forme graphique influence la sélection et la transmission des contenus, il s'agit de détailler le rapport de naturalisation. Il est composé de huit rubriques (qui ne correspondent pas aux questions posées en entretien, mentionnées ci-dessus), dont les sept premières ont pour titres respectifs "Identité», "Type de titre de séjour et validité», "Domicile en Suisse et dans le canton", "Interventions policières/condamnations antérieures/peines infligées à des mineurs", "Poursuites et dettes», "Situation fiscale», "Intégration dans la société suisse». Une "Synthèse de l'intégration» est proposée à la suite de ces sept rubriques.

En reprenant la distinction légale entre critères formels et critères matériels, on constate que les six premières rubriques concernent des aspects formels qui revêtent un caractère informatif. Les données qui y sont consignées sont compilées à partir des différents documents produits par la personne candidate. Chaque question se termine par des cases à cocher qui synthétisent sa situation. La septième rubrique, qui porte sur l'intégration dans la société suisse, relève de critères qualifiés de matériels. Elle revêt un caractère évaluatif qui se décline en quatre sections ( Respect de la sécurité et de l'ordre public», "Respect des principes fondamentaux de la Constitution", "Aptitude à communiquer dans une langue nationale», "Volonté de participer à la vie économique ou d'acquérir une formation»). $\mathrm{M}^{\mathrm{me}}$ Justine souligne la spécificité de cette rubrique en mentionnant la difficulté d'une évaluation objective et la nécessité de recourir aux sentiments pour la remplir:

29. Denis et Pontille, 2010; Grosjean et Lacoste, 1998; Latour et Woolgar, 1988. 
Un casier judiciaire, vous l'examinez, il est vierge, il n'est pas vierge. Là, vous ne pouvez pas avoir de sentiments. Mais au niveau de l'intégration, vous avez quand même votre culture, vous avez quand même vos sentiments personnels qui jouent un rôle, même s'il est petit. [...] Mon point de vue personnel, c'est que, sur l'intégration, on ne peut pas avoir que des points objectifs. Voilà. Il y a quand même une petite [part] qui vient de nous-mêmes. (Février 2015)

Chaque section doit être complétée par une coche qui indique si les critères sont remplis (oui/non). Un espace, facultatif, permet de commenter un point particulier. En plus de ces réponses exclusives, une évaluation distincte de l'expression et de la compréhension orale est proposée dans le rapport d'entretien, selon une échelle comportant quatre possibilités: «insuffisante», "suffisante», "satisfaisante», «bonne/très bonne». Lors de la recherche, cette évaluation reposait sur la libre appréciation des collaboratrices et des collaborateurs du Service de la cohésion multiculturelle, qui se basaient sur leurs propres connaissances linguistiques pour évaluer les compétences de compréhension et d'expression des personnes candidates. La loi en vigueur dès le $1^{\text {er }}$ janvier 2018 modifie cette répartition des tâches, puisqu'elle formalise l'évaluation des compétences linguistiques en la déléguant à des écoles de langues certifiées. Il n'est pas certain que cette formalisation résolve tous les problèmes potentiels d'interprétation qui se sont donnés à voir lors du terrain:

Dans un cas, pour nous [Service de la cohésion multiculturelle], c'était marqué "suffisant», pour eux [commune], ça ne l'était pas forcément. Donc on a déjà produit un document, [...] l'idée c'était de le transmettre aussi aux communes [...] en disant "voilà ce qu'on estime quand on dit suffisant, voilà ce que ça veut dire» [...]. On a tous une conception différente de ce que ce que c'est d'être suffisant, en termes de communication [...], certains critères et que, si on estime suffisant, c'est pour $\mathrm{X}$ raisons. Ça ne veut pas dire que la personne parle super bien français, qu'elle pourra tout comprendre tout de suite, mais qu'elle est capable de communiquer. (M. Corentin, avril 2015)

La dernière rubrique propose, sur le même système de cases à cocher, une synthèse qui permet d'évaluer l'intégration comme «consolidée», "en consolidation» ou «initiale». Le formulaire 
précise quels critères doivent être remplis pour chacune de ces catégories. Si les quatre critères sont remplis, le rapport conclut à une "intégration consolidée», comme c'est le cas ci-dessous:

\section{EXTRAIT DE LA SYNTHËSE D'UN RAPPORT D'INTÉGRATION}

\section{Synthèse de l'intégration}

Intégration consolidée (critères remplis $7.1 ; 7.2 ; 7.3 ; 7.4)$ : Intégration en consolidation (critères remplis $7.1 ; 7.2 ; 7.3$ ): Intégration initiale:

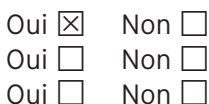

Ainsi, les rapports d'enquête doivent rendre compte des observations faites sur l'état d'intégration selon des rubriques définies, traduites par un système de coches. Les commentaires permettent d'intégrer des renseignements portant sur les trajectoires professionnelle et personnelle, qui se voient ainsi inscrits dans le dossier. Ces renseignements deviennent des indices, selon un glissement manifeste dans les intitulés du formulaire: la synthèse des quatre sections de la rubrique 7 devient, au final, la «synthèse de l'intégration" elle-même, et elle est finalement évaluée, comme on le verra ci-dessous, comme reflétant «l'intégration» en soi dans les décisions administratives postérieures.

L'analyse de ce rapport montre qu'au-delà de leur fonction informative, les documents sont des instruments de classement et de catégorisation. Leur forme schématique ou synthétique (l'emploi, par exemple, de cases à cocher) rend manifeste le travail de qualification ${ }^{30}$ effectué par les agents de l'État, qui doivent identifier des situations selon des catégories préétablies et à partir de l'accumulation de différentes pièces justificatives. À travers la fabrication du dossier, l'action administrative transforme un cas polyphonique en un cas maîtrisable inscrit dans une trajectoire bureaucratique prédéterminée ${ }^{31}$. Indispensables dans une perspective institutionnelle, les processus de catégorisation ont pour effet de simplifier les parcours individuels en les désindividualisant et en les généralisant. Ces processus contribuent à réduire l'intégration, en la matérialisant par les coches, à un recueil de signes ou d'indices apparemment objectifs. Plus généralement, les formulaires et autres écrits qui constituent peu à peu le dossier reconfigurent les

30. Weller, 2013.

31. Berg, 1996; Maskens, 2015; Mayère, Bazet et Roux, 2012. 
identités administratives à partir des spécificités individuelles pour produire des dossiers simples ou complexes, permettant ou non de prendre une décision positive (voir plus loin, 8.3).

Ni bon ni mauvais en soi, cet effet de catégorisation est inhérent à la logique administrative. Il influence les prises de décision lorsqu'il est amplifié par les routines professionnelles d'écriture. C'est le cas de l'emploi de formules toutes faites qui effacent progressivement la particularité de chaque demande. Cet effet de routine fait, dans certains cas, l'objet de plaintes de la part des candidates et candidats qui s'estiment lésés par le processus administratif de généralisation: «Moi, je dis que c'est une routine, une sorte de routine dans la gestion de dossiers, il y a des fois, on reçoit la même lettre, c'est copiécollé, et puis il y a des fois même [...] des lacunes dans les lettres" (M. Bruno). La généralisation peut même être perçue comme diluant la spécificité personnelle: «Moi, je trouve que mon dossier c'est copié-collé [du dossier d'] une autre personne. J'ai l'impression que c'est ça» (M. Gilbert). Le rapport et, par extension, le dossier sont réputés représenter l'individu, mais la suite d'opérations administratives agençant les différents documents et renseignements opère une disjonction progressive, dont résulte une simplification identitaire. Ainsi, à travers la multitude de documents demandés et générés par la procédure, les processus bureaucratiques façonnent des pièces, les indices, les découpent, les liment et les agencent afin de créer une image générale qui corresponde aux attentes légales et administratives. Les institutions produisent la personne candidate par le biais de leur graphie, elles l' «écrivent»: l'image qui émerge des dossiers ainsi produits n'est pas un reflet fidèle, mais bien une création bureaucratique basée sur la représentation idéalisée du bon dossier.

Par ses contraintes matérielles, le formulaire guide le traitement des informations en les sélectionnant, en les structurant selon un système qui leur confere une identité spécifique ${ }^{32}$. Le formulaire est plus qu'un outil, sa matérialité et son contenu sémantique en font un objet performatif: "Tous les phénomènes de mise en forme par le tracé, par la mise en page, jusqu'aux choix typographiques sont susceptibles de porter des significations et de participer à l'effectuation d'un énoncé performatif.» ${ }^{33}$ Les contraintes induites par la matérialité même du formulaire témoignent d'une volonté de

32. Études de communication (2009), vol. 33, p. 13.

33. Fraenkel, 2006. 
canaliser l'écriture selon des modèles préétablis, admis et attendus, et d'établir des catégories prédéterminées répondant à un besoin de lisibilité ${ }^{34}$. Au final, signatures et tampons qui attestent de l'authenticité d'un document administratif et, partant, de sa validité, sont porteurs d'une "force graphique» permettant à l'acte textuel de devenir performatif ${ }^{35}$. Pareils processus sont particulièrement visibles dans la prolifération des cases à cocher. Par les rubriques et le type de réponses proposé, le formulaire modélise et standardise la présentation de la réalité. Ce faisant, il induit une conduite et inscrit l'usage ${ }^{36}$. Ainsi, la construction et le format du «rapport d'intégration» infléchissent son contenu. Ils reflètent les représentations de l'intégration, les attentes face à la manière adéquate de l'évaluer. Outil de travail incontournable, le formulaire valide et transmet ces représentations de ce que l'intégration devrait être à l'ensemble des professionnelles et professionnels de la naturalisation - une standardisation qui entraîne une schématisation dans le mode de réponse, voire des automatismes ${ }^{37}$ (voir chapitres 6 et 7).

Le format du document, le contenant, influence la sélection des informations, notamment en imposant de réduire, pour des questions d'espace, la diversité des trajectoires personnelles et des énoncés. Le rapport d'intégration impose un style d'écriture simple, voire schématique, qui contribue à standardiser la complexité de la "réalité». La circulation, entre différents services institutionnels, de ces réécritures les transforme, puis les stabilise. Simplification, transformation ${ }^{38}$, puis sédimentation des énoncés sont le fruit de pratiques professionnelles transmises implicitement et induites par la procédure de naturalisation.

Les actrices et acteurs administratifs décrivent les routines comme des habitudes, des automatismes, soulignant ainsi positivement les contraintes du travail administratif sur qui l'effectue. Les procédures sont décrites de manière technique, en reprenant les consignes détaillées du Manuel de naturalisation, rédigé par le Secrétariat d'État aux migrations, dont l'objectif est d'«aider les collaboratrices et collaborateurs à répondre aux exigences élevées de leur tâche qui consiste à mener les procédures de naturalisation

34. Ehrlich, 2015.

35. Fraenkel, 2006.

36. Akrich, 2010.

37. Darling, 2014.

38. Lowenkron et Ferreira, 2014. 
rapidement et à prendre une décision juridiquement correcte» ${ }^{39}$. Malgré tout, les membres du Service de la justice ont éprouvé de la difficulté à expliquer le processus qui leur permet de définir si une personne est, ou n'est pas, intégrée: «Ça fait vingt ans que je travaille [dans ce domaine] et je me pose plus de questions qu'au début, pour savoir ce que c'est, l'intégration" ( $\mathrm{M}^{\mathrm{me}}$ Justine, décembre 2014). Comme on va le voir dans la section suivante, les agentes et les agents de l'État travaillent activement à produire du sens à partir de cette notion floue et problématique qu'est l'intégration. Cette activité constitue le processus décisionnel, dans lequel les informations retenues lors de l'enquête d'intégration et leur traduction dans le rapport se voient conférer un statut spécifique.

\subsection{CLORE LE DOSSIER}

Les trajectoires administratives s'achèvent sur une prise de décision, qui peut être positive ou négative au regard de la demande initiale. À cette étape finale correspond le dépôt du dossier physique dans les archives, et, depuis juin 2014, la clôture du cas dans GESTSTAR. Outre la dimension administrative, qui relève du canton, le processus décisionnel implique les trois niveaux politiques suisses - la commune, le canton et la Confédération - liés les uns aux autres par un mécanisme de cascade, puisque chaque instance se fonde sur ce que les précédentes ont rassemblé, sélectionné, synthétisé, décidé.

Ce mécanisme est compliqué: il est ardu d'identifier qui décide de quoi, qui influence qui. Lors des entretiens, nos interlocutrices et interlocuteurs, quel que soit leur statut, ont désigné de manière quasi unanime d'autres comme ayant un pouvoir décisionnel. Peu surprenant dans la bouche des employées et employés occupant des postes subordonnés, ce positionnement est plus inattendu de la part des personnes élues ou des responsables des services. Il peut se comprendre de trois manières: ces déclarations doivent être lues, tout d'abord, en rapport avec la nature du pouvoir institutionnel dans les démocraties occidentales. Elles sont aussi en lien avec le système administratif et politique suisse, dans lequel les demandes de naturalisation sont traitées par différentes instances. Finalement, elles reflètent la fragmentation du travail d'évaluation et de prise de décision dans la procédure. Cette section approfondit ces trois

39. Manuel sur la nationalité, chapitre 1, p. 1, https://www.sem.admin.ch/dam/data/ sem/rechtsgrundlagen/weisungen/buergerrecht/hb-bueg-kap1-f.pdf. 
dimensions en analysant la manière dont la prise de position détermine le résultat de la procédure, mais aussi ce qui est dit de la responsabilité décisionnelle.

\subsubsection{DU POUVOIR DE DÉCIDER}

«L'économie émotionnelle» du travail administratif dans les démocraties (les discours du feeling, de l'empathie) a comme particularité, non pas de "mettre en scène", mais au contraire d' "aplatir le caractère hiérarchique de la relation" administrative: sa raison d'être réside «dans la lutte contre les signes d'inégalité qui sont exigés par les fonctionnaires d'autres régimes bureaucratiques ${ }^{40}$. Cette analyse permet de comprendre la raison pour laquelle, dans les entretiens de recherche, la question du pouvoir des agents de l'État s'est faite évanescente.

Cela est particulièrement visible dans l'examen du rapport sur l'intégration établi par le Service de la cohésion multiculturelle. Le rapport joue un rôle central dans la procédure, car il porte sur l'évaluation d'un processus évolutif. C'est en fonction de la coche finale que l'intégration est signifiée comme étant suffisante ou insuffisante, consolidée ou en voie de consolidation. Le poids de cette option se fait sentir dans l'octroi du préavis communal lors de la première phase de la procédure:

[Le préavis], c'est vraiment facile, car le préavis suit ce que dit le COSM. C'est purement le COSM, il y a trois cases à cocher dans leur formulaire, si l'intégration est consolidée, c'est tout bon sans discussion, si c'est au milieu, intégration en cours, ou en bas, intégration initiale, on cherche à avoir plus de détails. (M. Noël, lors d'un entretien avec une Commission communale des naturalisations et des agrégations, avril 2017)

Or, selon la convention, le Service de la cohésion multiculturelle est responsable des enquêtes d'intégration tout en ne possédant aucun pouvoir décisionnel. Cet élément est d'ailleurs souvent mis en évidence par le personnel de ce service. Quand il rappelle que c'est "le Service de la justice [qui] s'occupe du dossier", M. Corentin souligne que "c'est eux qui gèrent. C'est eux qui ont la responsabilité», et que le Service de la cohésion multiculturelle

40. Hertz, Martin et Valli, 2004, p. 230. 
«n'est pas un organe décisionnel». Son collègue renchérit en disant que le Service de la cohésion multiculturelle est chargé de définir le «degré d'intégration de la personne» et non de déterminer si la personne est suffisamment intégrée:

En tant que service chargé de l'intégration et chargé, aussi, de déterminer l'intégration de la personne, nous déterminons le degré d'intégration de la personne. Ça ne veut pas dire quand même que nous forçons l'autorité de décision à donner la naturalisation, non! Nous, nous pouvons dire si telle personne est intégrée, maintenant. Est-ce que son degré d'intégration donne le droit à la naturalisation, ça, c'est une autre question. (M. Costin, septembre 2014)

Les employées et employés du Service sont chargés de rechercher des indices, de les rassembler et de les évaluer par le biais du formulaire idoine; selon les entretiens, leur responsabilité s'arrêterait là. Dénués de pouvoir décisionnel officiel, que conférerait par exemple la capacité d'apposer un tampon disant que la personne est suffisamment ou insuffisamment intégrée, elles et ils se considèrent comme de simples rédactrices et rédacteurs. En effet, le Service insiste pour souligner que l'intégration est un processus et non un état. Cette subtile distinction s'est retrouvée au centre d'un quiproquo lors d'un échange d'e-mails entre l'équipe de recherche et une employée du Service. Voulant vérifier la date à partir de laquelle le COSM était devenu responsable des rapports d'intégration, une personne de l'équipe a reçu comme réponse que «le COSM ne fait pas de rapports d'intégration». Comme nous venions d'analyser en profondeur le formulaire et de participer à une formation continue sur comment les remplir, la chercheuse, quelque peu abasourdie, a insisté, demandant à sa correspondante de se renseigner auprès de ses collègues. La collaboratrice du COSM est revenue ainsi, par e-mail: "Voilà, renseignement pris, c'est plus clair. En fait, chez nous, au COSM, on ne parle pas de rapports d'intégration et comme je ne m'occupe pas de cela, c'est pour cette raison que j'étais un peu perdue» (e-mail du 2 octobre 2015). Cependant, c'est bien en "suivant» le rapport, donc en regardant quelle case a été cochée, que la commune émet un préavis positif ou négatif.

Lors des entretiens, les collaboratrices et collaborateurs des autres services cantonaux ont également régulièrement insisté sur cette absence de pouvoir en soulignant le caractère bureaucratique 
de leur travail: au quotidien, on se borne à «appliquer la loi», on "suit ce que dit la loi", comme si cette dernière traçait un chemin balisé. Les agentes et les agents de l'État tendent à nier ou ignorer la présence comme l'ampleur de leur capacité d'infléchir la trajectoire d'une demande. Pourtant, ils possèdent un véritable pouvoir discrétionnaire, au sens proposé par Laurens ${ }^{41}$ : leurs pratiques administratives se déploient dans un contexte complexe d'injonctions contradictoires, fait de tiraillements, de considérations morales et privées, où

l'expression d'un "for intérieur» (Chevallier 1995) n'est pas totalement disqualifiée dans les interactions qui se nouent autour de l'instruction des dossiers. Si historiquement la légitimité bureaucratique s'est construite par la relégation dans un espace "privé» des émotions et des opinions personnelles, faire de l'idéal légalrationnel, promu par l'institution, la réalité totale des interactions à l'intérieur de l'État serait faire fi d'une réalité quotidienne où des espaces d'expression subsistent. ${ }^{42}$

Face à ces injonctions contradictoires, au manque de définition univoque de l'intégration, et dans un contexte démocratique où les signes de pouvoir tendent à être aplanis, le pouvoir discrétionnaire des agentes et agents de l'État apparaît comme quelque chose de négatif, de "sale»" ${ }^{43}$, c'est-à-dire un élément qui n'est pas à sa place, qui perturbe l'ordre des choses en polluant l'ordonnance du monde. La marge de manœuvre décisionnelle souligne le caractère asymétrique des relations entre professionnelles et personnes requérantes. Elle rappelle que même si les premiers attendent un retour des seconds pour qualifier leurs compétences professionnelles, les outils à disposition des uns et des autres ne sont pas les mêmes, ni les enjeux: potentiellement, pour les candidates et candidats, il s'agit de "perdre sa vie", comme le dit M. Bruno (mai 2014). Les personnes qui déposent leur dossier n'ont que peu d'impact sur l'établissement des règles de ce jeu sérieux et de ses hasards ${ }^{44}$. Les agentes et les agents de l'État construisent du sens et de la cohérence qui leur permet «d'organiser la polyphonie, [de] réduire

41. Laurens, 2008.

42. Ibid., p. 31.

43. Douglas, 2005.

44. Cretton, 2012. 
la dissonance, [de] produire la cohérence ${ }^{45}$. Garantir l'équité en la ramenant à la bonne volonté, à la volonté de faire de son mieux peut être suffisant pour les professionnelles et professionnels:

On peut parfaitement être transparent à mon avis, ou quasiment parfaitement, parce qu'on essaye de travailler équitablement, on fait de notre mieux. Cela veut dire que même sans prendre un dossier spécifique, on peut dire si c'est un dossier comme ci ou comme ça, ainsi de suite. Et s'il y a un cas comme ça, on sait qu'on fait comme ça, et ainsi de suite. [Le candidat] sentira, au travers de notre discours, au travers de notre contact, qu'on fait pour tout le monde la même chose, parce qu'à la limite on n'aura même pas demandé son nom, on ne lui aura même pas demandé ses caractéristiques, ça veut dire qu'il saura qu'il sera traité comme les autres, il saura qu’il sera traité de manière équitable. (M. Jules, avril 2015)

Les personnes candidates doivent quant à elles suivre les règles dictées par les institutions légales et bureaucratiques qui encadrent et déterminent leur agentivité d'une manière beaucoup plus contraignante que pour le personnel administratif: pour elles, faire de leur mieux n'est pas automatiquement suffisant.

Les membres du personnel de l'État ont d'autant plus de peine à reconnaître leur pouvoir individuel que la procédure met l'accent sur la systématisation du traitement et l'interchangeabilité des agents de l'État les uns avec les autres. Pourtant, certains énoncés trahissent une conscience de ce pouvoir: "On fait quand même notre travail, hein. On n'abuse pas de notre position", dit M. Jules (avril 2015). Une fois de plus, c'est l'équilibre qui est important, s'il n'y a pas d'abus de la position, ce pouvoir peut être reconnu, il fonctionne de manière performative.

Le pouvoir décisionnel réapparaît explicitement dans des situations spécifiques, lorsqu'il peut être utilisé à des fins morales plutôt que dans le cadre d'un simple exercice administratif:

Quelle est la responsabilité des membres de la famille [dans l'intégration]? [...] On ne parle pas de l'enfant hein, on parle d'un adulte. On parle de l'épouse ou de l'époux, en l'occurrence souvent c'est l'épouse. Non, mais c'est des cas typiques. Il faut ajouter

45. Krieg-Planque et Ogier, 2010. 
l'aspect genre et l'aspect pouvoir là-dedans! Soyons clairs, parce que souvent dans [...] ces cas [...], c'est la femme, qui est dans ce cas, qui n'a pas de liens, qui ne participe à rien, alors la question c'est: Est-ce que c'est son choix ou pas, enfin toutes ces questions-là, mais, jusqu'où on entre nous-mêmes dans l'intimité des gens pour savoir? Mais on peut quand même se poser aussi la question du soutien plutôt que la responsabilité [...]: quel est le soutien apporté, pour permettre à quelqu'un qui le souhaiterait, de participer à [des cours de langue]. (Entretien avec un membre de la commission chargée de l'évaluation préalable des demandes, juin 2014)

M. Corentin remarque que, "dans un couple, suivant la culture des personnes, même si l'épouse voudrait le faire [prendre un cours de français], elle n'en a pas la possibilité parce que son mari ne lui en laisse pas vraiment la possibilité et sa culture ne lui laisse pas la possibilité de se battre contre son mari» (avril 2015).

Dans pareils cas, le pouvoir des agents de l'État n'est plus tu, mais au contraire affirmé. Il devient une ressource valorisée pour lutter contre les asymétries de pouvoir supposées présentes dans ces familles non suisses. Le pouvoir trouve sa place, car il contribue à faire régner le bon ordre, en renvoyant les inégalités de genre à un contexte autre, construit comme lointain et incompréhensible, dans lequel les comportements seraient dictés de manière déterministe par des coutumes et traditions dépassées ${ }^{46}$. En employant un discours rappelant la nécessité de sauver les femmes étrangères de structures patriarcales imposées par leurs maris, pères et frères ${ }^{47}$, les agents de l'État survisibilisent les inégalités sociales chez les autres et renforcent l'idée reçue selon laquelle elles seraient moindres, voire inexistantes, en Suisse. Cela leur permet de reconnaître un pouvoir institutionnel qui devient légitime, et non sale et polluant, car il est mis au service d'un ordre social moralement supérieur qu'il convient de défendre.

La difficulté à identifier les tournants critiques pour les trajectoires de dossiers que sont les prises de décision tient à la structure de la procédure. Dans le chapitre 5, le détail des étapes par lesquelles passent les dossiers a permis de comprendre la répartition de la procédure en deux phases. La première est la demande de l'autorisation fédérale, la seconde celle de l'octroi, par la commune, puis par le 
canton, de leur droit de cité respectif. La nationalité découle de ces deux octrois. La lisibilité de cette structure est compliquée par le fait que les trois niveaux administratifs et politiques interviennent dans les deux étapes. Ces particularités expliquent la difficulté à identifier les autorités décisionnelles; mais elles sont aussi des révélatrices des mécanismes de prise de décision institutionnelle.

Le travail des agentes et des agents de l'État se caractérise par une segmentation des tâches qui brouille une vue d'ensemble des actions respectives des uns et des autres. À cela s'ajoutent des directives et des attentes qui sont contraignantes au regard de cette chaîne d'activités. Celle-ci contribue à filtrer les demandes en examinant de manière détaillée leur recevabilité. Selon $\mathrm{M}^{\mathrm{me}}$ Justine, cette manière de procéder permet de répondre aux directives du Secrétariat d'État aux migrations et de diminuer le nombre de «dossiers négatifs» qui lui sont envoyés: "la plupart des dossiers sont favorables, c'est-àdire qu'on transmet très peu de dossiers négatifs parce que l'Office fédéral nous a demandé de les éliminer; ils n'aimeraient pas, ils n'aimeraient pas avoir à les traiter" (décembre 2014).

On le voit, le langage interne au service distingue entre dossiers simples et dossiers complexes. Mais ce langage se modifie dans les interactions avec les agents de l'État d'autres niveaux. Dans la transmission au Secrétariat d'État aux migrations, le discours glisse vers l'évaluation entre des dossiers qui seraient positifs et des dossiers présentés comme étant "négatifs», alors même qu'il n'y a encore aucune décision prise. En effet, le Secrétariat d'État aux migrations intervient pour clore la demande d'autorisation fédérale, qui n'est pas une décision quant au contenu, mais qui autorise commune et canton à statuer sur la demande de droit de cité. Ces dossiers dits "négatifs" sont des demandes qui sont maintenues alors que l'un des préavis au moins est négatif. Dans pareils cas, la personne candidate se voit informée du fait que la demande a peu de chance d'aboutir et on lui conseille de «régler sa situation" ( $\mathrm{M}^{\mathrm{me}}$ Justine, février 2015). Ces mises en garde ont pour but de leur faire reconsidérer le bien-fondé de leur demande. Comme montré précédemment, accepter ce type de conseil et faire preuve de patience sont des qualités dont les «bonnes» requérantes et les «bons» requérants devraient faire preuve. Et donc, paradoxalement, les personnes dont la demande a peu de chance d'aboutir attestent de leur intégration en adoptant une bonne attitude, faite «d'acceptation» et «d'humilité». 
Ces stratégies administratives permettent aussi de conserver des taux d'efficacité élevés. Les services qui ne traitent que de cas simples et positifs peuvent travailler plus rapidement. De même, les cas visiblement négatifs, dans lesquels les critères formels ne seraient pas respectés par exemple, sont faciles à traiter: "Il y a les cas blancs, les cas noirs. Ça, c'est facile. Après il y a ceux qui sont sur la tranche, à la limite, à la frontière» (M. Jules, avril 2015). Ce sont ces derniers qui nécessitent un investissement en temps et en énergie. Cet investissement influence la rapidité de traitement, par laquelle l'efficacité de l'administration est jaugée. C'est pourquoi la sélection a lieu en amont, par l'élimination de demandes «négatives ». Pareille sélection garantit en outre des statistiques d'acceptation élevées, puisque les cas "sur la tranche» ne sont présents qu'en nombre restreint. Ces processus administratifs ont comme conséquence qu'il y a peu de décisions «vraiment négatives»:

Déjà, je dirais qu'il y a peu de décisions vraiment négatives, hein! Il y a des refus d'autorisation fédérale, ça, c'est une chose, mais au niveau de la décision et communale et cantonale, il y a peu de refus. En pourcentage, c'est un petit pourcentage donc, et ensuite sur ces refus, tous ne font pas recours contre la décision du Conseil d'État qui refuse la naturalisation. ( $\mathrm{M}^{\mathrm{me}} \mathrm{Justine}$, avril 2015)

\subsubsection{LA PROCÉDURE "RECOMMANDABLE 》 D'UN CANTON "LIBÉRAL »}

En mettant en œuvre la loi sur la nationalité, le personnel administratif endosse, traduit et définit la posture du canton à l'égard des personnes étrangères. Or, Neuchâtel jouit d'une réputation de libéralisme et de tolérance, qui n'est pas anodine pour comprendre les pratiques décisionnelles. En effet, les actrices et les acteurs administratifs interprètent et traduisent la notion d'intégration selon leurs expériences personnelles et sur la base de systèmes d'interprétation qui orientent leurs pratiques ${ }^{48}$.

Inhérentes au contexte social, politique, législatif et historique dans lequel elles se déploient (voir chapitre 3), les conceptions de l'intégration sont prises dans un mouvement pendulaire: les représentations des agents et agentes de l'État infléchissent leurs pratiques; et, en retour, leurs pratiques façonnent leurs représentations. Pour comprendre le travail du personnel de l'État neuchâtelois, il importe

48. Jodelet, 1989. 
par conséquent de considérer le système administratif et politique suisse. Ce dernier est marqué par une grande autonomie cantonale dans différents domaines, et notamment dans les questions connexes des politiques d'intégration, de naturalisation et de traitement institutionnel, social et administratif des étrangères et étrangers (voir chapitres 4 et 5). Cette autonomie a pour corollaire le développement d'une culture de comparaison entre les cantons, à laquelle contribuent les rapports établis sur mandat des autorités fédérales. Leurs résultats sont diffusés au sein des administrations fédérales et cantonales, ainsi que de manière plus générale par les médias traditionnels, par exemple au lendemain de votations. La culture politique d'autonomie cantonale façonne donc, localement, des manières de faire collectives répondant à la double injonction du besoin de se distinguer tout en restant conforme ${ }^{49}$. Dans les administrations, cette double injonction se traduit par des énoncés qui endossent les réputations spécifiques et présentent les pratiques locales comme celles qui suivent le plus fidèlement l'esprit du cadre légal national ${ }^{50}$.

La réputation de tolérance des politiques d'intégration et de naturalisation du canton de Neuchâtel est fondée sur un audit comparatif appréciant les possibilités de participation offertes aux non-Suisses à la vie publique et politique locale ${ }^{51}$. La dimension sociale est fondée sur le soutien institutionnel, tant symbolique que financier, aux associations dites de migrantes et migrants et aux cours de langues et cultures qu'elles dispensent. La dimension politique inclut l'existence de droits civiques octroyés aux personnes non suisses ainsi que les réglementations en matière de naturalisation. Dans le canton de Neuchâtel, les personnes étrangères ont le droit de vote en matière communale depuis 1850. À l'origine, était étrangère toute personne venant d'un autre canton. Cette définition légale s'est modifiée pour désigner les personnes n'appartenant pas à la Confédération suisse, sans que le droit de vote ne soit remis en question, conférant à Neuchâtel une image de pionnier pour les droits des étrangères et étrangers:

[Favoriser la cohésion sociale], c'est le point de vue du Canton de Neuchâtel sur ces questions [d'intégration], c'est pour ça aussi que, extérieurement et dans les milieux académiques, on est

49. Bourdieu, 1979.

50. Voir aussi Lavanchy, 2011; Lavanchy, 2012.

51. Cattacin et Kaya, 2001. 
perçu, $[\ldots]$ je pense à juste titre parce qu'on a ces bases solides, comme des pionniers [...] parce qu'effectivement, le Canton de Neuchâtel, une de ses valeurs, c'est d'être une république libérale. (M. Corentin, avril 2015)

N'ayant pas produit de données similaires sur les pratiques dans d'autres cantons, notre ouvrage n'a pas pour objectif de confirmer ou d'infirmer la réalité de la réputation neuchâteloise de tolérance et de libéralisme. Mais il importe d'inclure ce contexte dans l'analyse pour comprendre le cadre dans lequel s'inscrit le travail d'évaluation administrative. La réputation colore en effet les pratiques institutionnelles: elle est souvent mentionnée de manière explicite par les professionnelles et professionnels de la naturalisation interviewés dans le cadre de la recherche. Le positionnement affirmé du canton devient donc un outil de distinction efficace, dans la mesure où il fonde et légitime certaines actions. Le personnel administratif neuchâtelois invoque ainsi la réputation de tolérance comme principe explicatif de certains de ses choix, en soulignant que "c'est l'ambiance générale qui prévaut» (M. Corentin, avril 2015). Les références récurrentes au libéralisme ne signifient pas pour autant que cette réputation ait une signification univoque. Elle peut en effet servir à légitimer des choix apparemment contradictoires au cours de la procédure. Par exemple, les membres d'une Commission des naturalisations et des agrégations l'ont invoquée pour étayer des positions contradictoires, les uns soutenant qu'il était justifié, sur cette base, d'octroyer un préavis communal favorable à une demande, alors que d'autres s'y référaient pour alléguer d'un préavis négatif, arguant qu'elle risquait d'être bradée si «tout et n'importe quoi» devait être accepté ( $\mathrm{M}^{\mathrm{me}}$ Natacha, mars 2017).

Le Service de la cohésion multiculturelle représente et ancre institutionnellement la réputation neuchâteloise de libéralisme et de tolérance. Elle a été personnifiée par la figure de Thomas Facchinetti, artisan charismatique de cette politique volontariste d'intégration depuis 1990, qui a dirigé jusqu'en 2012 le Bureau des étrangers (BDE), devenu le Service de la cohésion multiculturelle, marquant durablement la politique d'intégration de ce service et du canton (voir chapitre 3). La formalisation de la participation du Service de la cohésion multiculturelle à la procédure de naturalisation est réglée par une convention signée avec le Service de la justice. La convention établit les règles de collaboration et les compétences respectives 
des deux services dans la procédure, stipulant notamment que «le JUST-Naturalisation délègue au COSM-Naturalisation les enquêtes et l'établissement des rapports d'intégration et de naturalisation ainsi que les rapports concernant les procédures d'annulation de la naturalisation facilitée» (art. 1 de la Convention). Elle précise en page 4 que les rapports doivent «mentionner le degré d'intégration estimé par le COSM-Naturalisation selon des degrés préétablis et communiqués au JUST. Si l'intégration est jugée insuffisante, un texte en explique clairement les motifs dans le rapport».

Des entretiens de recherche comme des observations sur le terrain, il ressort que la mise en application de la loi passe par une lutte contre une évolution légale perçue comme de plus en plus restrictive et, partant, contraire à l'esprit cantonal, puisque la nouvelle Constitution cantonale adoptée en 2000 a étendu le droit de vote du niveau communal au niveau cantonal. Parlant des "durcissements continuels» de la loi fédérale, M. Corentin estime qu'il faut «se battre quand même contre soi-même pour continuer à garder [la pratique libérale du canton de Neuchâtel] bien en tête » (avril 2015). Cette lutte contre soi-même est compliquée en raison $\mathrm{du}$ «trend qui est dans le durcissement de partout, et puis cette position elle doit être défendue, continuellement, surtout quand ça va mal, surtout quand il y a des difficultés; [...] l'opinion publique, qui a toujours été plutôt ouverte à Neuchâtel, tend à l'être moins " (M. Corentin, avril 2015). À la spécificité du positionnement réputé libéral du canton répond la spécificité de la procédure, elle aussi considérée comme étant «exemplaire» comparativement aux procédures en vigueur dans les autres cantons:

Je ne sais pas si vous avez vu, la Commission d'intégration du Canton de Fribourg a émis des recommandations sur la procédure de naturalisation [...], et ils mettent en avant le principe neuchâtelois, notamment parce qu'on ne multiplie pas les entretiens. (M. Jules, avril 2015)

Dans le même esprit, $M^{\text {me }}$ Michèle confie que «le Valais s'en tire bien aujourd'hui ", car justement sa procédure se rapproche de celle de Neuchâtel: elle est "efficace», avec un "traitement rapide des dossiers» et il y a "peu d'entretiens» (avril 2015). M. Jules ajoute: "Il y a beaucoup de cantons qui coupent les cheveux en quatre et c'est à se demander s'ils ne s'accordent pas, à eux-mêmes, trop 
d'importance!» L'efficacité de la procédure neuchâteloise se mesure aussi par rapport à ce qui se faisait "avant " : «Il y aura toujours [des personnes qui râlent], mais dans l'ensemble, les gens sont contents parce que ça durait beaucoup plus longtemps avant, et d'autres cantons sont beaucoup plus lents» (M. Jules, avril 2015).

Les comparaisons avec ce qui se fait ailleurs ne se limitent pas à la dimension cantonale. Elles se retrouvent dans les communes, de même qu'en lien avec des entités plus symboliques que formelles, comme "la Suisse alémanique» et "la Suisse romande»:

Je dirais que c'est ça le principal problème, on fait les choses chacun différemment [dans chaque commune]. On a des approches qui sont différentes et pourtant c'est la même loi. Et c'est encore pire si on compare en dehors du canton, avec nos voisins. En Suisse allemande, ils sont plus durs. Neuchâtel, on est gentil par rapport aux autres, on est des pionniers pour l'intégration. (M. Nasser, mars 2017)

Moi, je constate qu'il y a de toute façon une approche différente qu'on soit en Suisse romande ou qu'on soit en Suisse latine [par rapport à la Suisse alémanique]. Ça devient difficile, en Suisse alémanique, par rapport à l'étranger d'une façon générale. Mais même à l'intérieur d'une même région romande, on a des approches qui peuvent être aussi assez, assez, assez, je ne vais pas dire complètement à l'opposé, mais, mais avec des attitudes qui sont beaucoup plus sévères. Moi je prends par exemple Neuchâtel/ Fribourg, dans le domaine [...] des étrangers d'une façon générale, on sent que c'est quand même assez, assez dur [à Fribourg], j'entends [dans les réunions intercantonales] «la règle c'est ça et puis on l'applique!» (Mme Michèle, avril 2015)

Il n'est pas surprenant qu'outre l'efficacité, qui a fait l'objet de réformes ciblées, la question de la tolérance comme spécificité neuchâteloise apparaisse aussi lors de pareilles comparaisons:

Ça n’a rien à voir, moi [...] quand je vais à des réunions à Berne, on ne pratique pas pareillement. Tous les cantons ont des pratiques beaucoup plus dures, plus lourdes, plus restrictives aussi dans les conditions. Ils ont des lois où il y a plus de conditions que chez nous, ils sont déjà plus en accord avec ce qui va se faire avec la nouvelle loi. ( $\mathrm{M}^{\mathrm{me}}$ Juliette, avril 2015) 
Lallusion à une mise en pratique anticipée des préceptes de la «nouvelle loi» - la loi sur la naturalisation de 2014, en vigueur depuis le $1^{\text {er }}$ janvier 2018 - est significative. Lapplication des lois est aussi influencée par des éléments historiques, comme c'est le cas pour Neuchâtel et sa réputation de libéralisme et de tolérance. Ceci signifie que des cantons peuvent faire du zèle en anticipant des mesures légales plus restrictives, qui ne sont pas encore en vigueur, mais le seront sous peu. Anticiper des conditions limitatives peut être présenté comme la preuve d'une adéquation maximale avec la loi et son "esprit " ${ }^{52}$ ou, au contraire, comme c'est le cas ici, comme un acharnement qui n'a pas sa place dans une mise en application sérieuse du dispositif légal.

La tolérance ne signifie pas cependant un laisser-aller. Il faut savoir être "strict» dans certains domaines pour pouvoir se permettre la tolérance:

Et puis être à jour [dans le paiement de ses impôts], là il y a des interprétations. On doit être en règle avec les contributions, mais il peut y avoir des interprétations. Ça peut être avoir du retard, mais payer par arrangement, ça dépend les cantons en fait. La Confédération admet que si, pour délivrer l'autorisation, la personne a du retard, mais qu'elle respecte un arrangement de paiement, elle est à jour avec ses impôts, le Canton de Neuchâtel est plus strict. Voilà... (M ${ }^{\text {me }}$ Juliette, avril 2015)

M. Jules affirme d'ailleurs qu' «il ne faut pas être dupe», car il en va de la "crédibilité des institutions" (avril 2015). L'alchimie particulière consiste donc à trouver, une fois encore, ce délicat équilibre entre des injonctions contradictoires, en veillant à sauvegarder la réputation cantonale d'excellence, de "pionnier» et la valeur «recommandable» de sa pratique administrative (M. Corentin, avril 2015), mais aussi la crédibilité des institutions locales. C'est cet équilibre, symbolisé par l'importance de l'influence et du consensus, que nous allons analyser dans la dernière section de ce chapitre.

\subsubsection{LA FORCE DE L'INFLUENCE ET DU CONSENSUS}

Les traces écrites sont au cœur du dossier; sélectionnées par les services cantonaux, ce sont elles qui circulent entre les entités 
impliquées. Mais si elles sont présentées comme l'expression de faits par les autorités cantonales, elles ne sont pas toujours acceptées comme argent comptant par les communes. Les élues et les élus qui composent les Commissions communales des naturalisations et des agrégations ont eu à leur égard des discours très contrastés, affirmant d'emblée une proximité dont les services cantonaux seraient dénués:

Les dossiers, ce n'est que du formalisme administratif, du langage administratif, ils ne rencontrent pas les gens, des fois, on ne peut pas savoir avec les documents, il faut parler avec les gens, nous, on a la proximité. (M. Nathan, mars 2017)

La proximité se traduit par le fait que les candidates et candidats sont invités par les commissions à un entretien: «Les interviews, c'est crucial, car il faut dire que les dossiers, des fois, ils arrivent n'importe comment du canton» ( $\mathrm{M}^{\mathrm{me}}$ Natacha, mars 2017).

Les élues et élus communaux mettent en scène les différences entre un traitement administratif des dossiers et le traitement politique. En construisant le canton comme une entité en soi, les spécificités ou même les tensions entre les positions du Service de la cohésion multiculturelle et celles du Service de la justice sont gommées. Construite en opposition avec le canton et sa machinerie administrative, la commune est présentée comme une organisation à taille humaine, qui permettrait une réelle proximité avec les candidates et candidats. Les processus de généralisation, de simplification et de dépersonnalisation, qui résultent $\mathrm{du}$ - mais aussi permettent le - traitement administratif des dossiers sont rejetés au profit d'un autre type de savoir, la "connaissance des gens", fondée sur la rencontre interpersonnelle.

Les pratiques communales de naturalisation varient parfois fortement les unes des autres. Certaines Commissions des naturalisations et des agrégations interviennent lors des deux phases de la procédure; dans d'autres cas, ce sont les membres du dicastère responsable (une ou deux personnes parfois, selon la taille de la commune) qui sont chargés de donner le préavis communal dans la première phase de la procédure. Certaines communes, notamment les plus petites, convoquent systématiquement les candidates et candidats pour un entretien avant de décider de l'octroi du droit de cité. Pour les personnes ayant accès à la procédure simplifiée, 
l'entretien communal est le seul moment de rencontre interpersonnelle, ce qui explique probablement le fait que Johan considère que seule la commune est intervenue dans le traitement de son dossier (chapitres 6 et 7 ).

Élément capital dans la présentation du travail communal, qui apparaît tant dans les entretiens que dans les réponses aux questionnaires envoyés à toutes les communes, la proximité se traduit par un souci de chercher les "circonstances atténuantes", un vocabulaire emprunté au droit pénal et qui est utilisé à plusieurs reprises lors des entretiens avec les membres des commissions communales. Curieusement, cette même idée de chercher des «circonstances atténuantes" se retrouve au sein du Service de la cohésion multiculturelle, où les professionnels ont expliqué chercher s'il existait des «circonstances atténuantes» (M. Costin, septembre 2014) pouvant expliquer qu'une requérante ou un requérant ne remplisse pas totalement les critères d'intégration attendus. Il s'agirait selon les professionnels de ce service de "pondérer", de "donner des arguments ", ce qui peut permettre de "sauver des dossiers".

Pour la commune, il s'agit en quelque sorte de rectifier ce qui est perçu comme des approximations générées par un travail administratif routinier et peu précis. "Vous vous souvenez du dossier du jeune Éthiopien?" lance, lors d'un entretien collectif, le membre d'une commission à l'intention de ses collègues, qui acquiescent et relatent à plusieurs voix l'histoire suivante:

Le dossier, il montrait qu'il était au social; ses parents avaient divorcé, ils étaient venus en Suisse pour demander l'asile, le dossier quand on l'a vu, il disait que le jeune homme ne faisait rien, qu'il était au social. Puis on l'a entendu, c'était vraiment une belle histoire, il était au social, mais pour terminer ses études, il était au CPLN [Centre professionnel du Littoral neuchâtelois] et il voulait faire son service militaire. Il s'était vraiment intégré, le dossier était mal fait, nous on a pu voir tout cela lors de l'entretien, d'abord on était mitigés sur le dossier, puis on a vu que ça allait très bien, on a fait un préavis positif, et c'est bon maintenant, il est Suisse. (MM. Norman et Nasser, $\mathrm{M}^{\text {mes }}$ Natacha et Nadège, mars 2017)

Soulignons que la méconnaissance de ce que font exactement les autres niveaux politiques et administratifs concerne tous les acteurs : 
Je n'ai même pas connaissance des règlements communaux. Je ne peux pas vous dire, je ne sais pas quelle commune a vraiment un règlement sur la naturalisation, des dispositions sur la naturalisation. Je ne sais pas, chaque commune étudie un petit peu à son gré, les pratiques de toutes les communes, je ne les connais pas [rires et gêne]. [...] Simplement, l'obligation de la loi, c'est qu'elles aient une Commission des naturalisations, et puis maintenant, vous dire plus que ça, je ne pourrais pas [rires et gêne], je ne peux pas. (M ${ }^{\mathrm{me}}$ Justine, avril 2015)

Selon les élues et élus communaux, l'investissement individualisé dans les communes permet donc de reprendre les dossiers «mal faits", qui "arrivent du canton avec peu ou pas de commentaires. On se demande comment ils font leur travail. Après, on les appelle pour leur dire que ça ne va pas, et la seule chose qu'ils répondent, c'est qu'ils sont débordés" (M. Noël, avril 2017). Par exemple, pour contacter les témoins, les services cantonaux se contenteraient de faire un ou deux essais "pendant les heures de bureau", alors que c'est le soir que les témoins sont atteignables. Contrairement au personnel cantonal, les élues et élus communaux s'engageraient au-delà de ces horaires restrictifs, des routines administratives, prenant mieux en considération les spécificités de la vie quotidienne et s'investissant pour le bien public. De fait, les élues et élus font un travail de milice qui se déroule souvent en marge d'activités professionnelles, avec des réunions en soirée. Mais c'est surtout la rencontre, par le biais de "l'interview», qui compte: "L'interview, c'est quand même crucial, c'est le point déterminant» (M. Noël, avril 2017).

La proximité de la commune est revendiquée aussi pour mettre à l'aise les candidates et candidats :

Pour certains, [l'interview] c'est impressionnant, c'est comme un examen, mais nous on aimerait juste vérifier que c'est possible de discuter avec eux [...]. Des fois, ils n'arrivent même plus à dire quelques mots, parce qu'on est les sept [la commission au complet], là, en face d'eux, c'est difficile, comme à l'école, et certains ne sont pas allés à l'école depuis longtemps, ou même certains ne sont presque pas allés à l'école. ( $\mathrm{M}^{\mathrm{me}}$ Nadège, mars 2017)

Une de ses collègues souligne aussi : 
Les entretiens, c'est pour connaître les motivations, les loisirs, la connaissance... Ce ne sont pas des questions hyper précises, genre "est-ce que vous connaissez le nombre de communes ou de districts à Neuchâtel?». Ouais ce ne sont pas des choses de ce type-là, vraiment pas, hein. [...] Le canton de Vaud, c'est pas du tout comme [ce que nous faisons ici], le canton de Vaud, c'est très compliqué. ( $\mathrm{M}^{\mathrm{me}}$ Norma, avril 2017)

Cette philosophie reflète en quelque sorte celle du canton, soucieux d'endosser et de réaffirmer sa réputation de tolérance. Les entretiens doivent se dérouler "dans une bonne atmosphère", pour que les candidates et candidats se sentent "à l'aise», ce n'est pas un «examen» ( $\mathrm{M}^{\mathrm{me}}$ Nadège, mars 2017):

L'important dans l'entretien, c'est d'aller à la rencontre des gens, on attend d'eux qu'ils s'expriment. Dans certaines commissions dans d'autres communes, il y a plein de questions précises, ici, on va à la rencontre des gens. Ils s'attendent à un tribunal, mais vous voyez comme on est assis, ce n'est pas une salle avec plein de tables les unes en face des autres, ce n'est pas un examen, c'est une discussion. Il n'y a pas de canevas, c'est libre, c'est juste une discussion, mais la question des motivations, on la pose toujours, les raisons de la demande et les motivations, on commence par ça [...]. Pour les gens qui se sentent intégrés, pour eux ce n'est pas important, ça ne change rien, mais c'est pour la sécurité des enfants souvent, pour qu'ils puissent être protégés, c'est une formalité qu'ils font pour les enfants [...]. La naturalisation, c'est le point ultime, on est intégré, puis finalement on demande à être naturalisé, c'est naturel. (M. Nasser, mars 2017)

Bien qu'ils soient conscients du stress généré par la situation d'entretien, les membres des commissions communales rencontrés affirment que, pour qui «se sent intégré», les entretiens ne constituent qu'une "formalité». Pourtant, leurs propos montrent que ces rencontres sont loin d'être anodines pour les candidates et candidats.

Des fois, ils ont l'impression de passer un examen, ils nous demandent «mais pourquoi vous me demandez si je suis intégré, je suis comme vous, je suis né ici, il y a quelle différence?» et c’est 
vrai, ils ont raison, ils sont comme nous, des fois il n'y a même pas de différence de couleur de peau, on ne voit rien. (M. Norman, mars 2017)

Et même si les commissions veulent juste «discuter» pour "se faire une idée», elles ne peuvent «tout» accepter: "Mais quand même, on ne peut pas excuser tout, c'est important de ne pas se laisser avoir, il y en a qui jettent de la poudre aux yeux. Il y en a qui savent se vendre, [...] c'est vrai qu'il y a les beaux parleurs, mais on arrive à voir ça, dans l'entretien» ( $\mathrm{M}^{\mathrm{me}}$ Natacha, mars 2017). Ainsi, la rencontre ne permet alors pas tant de valider que d'identifier les "mauvais éléments", les personnes dont la naturalisation serait inadéquate malgré les apparences - que la constitution du dossier ne permettrait pas de dépister. La question des entretiens faits par les Commissions des naturalisations et des agrégations est importante, car même si les communes ont été intégrées à la fin du terrain, ce sont les seuls entretiens dans lesquels le pouvoir décisionnel est spontanément revendiqué: "C'est nous qui décidons" ( $\mathrm{M}^{\mathrm{me}}$ Nadège, mars 2017)» Cette affirmation a cependant été nuancée quelque peu: "Nous, on a un avis purement consultatif, comme commission. C'est le Conseil communal qui prend la décision après. Il pourrait ne pas suivre notre avis, mais ça ne s'est jamais produit» (M. Nelson, mars 2017).

Ces deux affirmations reflètent l'ambivalence des prises de décision. Les instances investies d'une autorité - ce sont elles qui apposent leur sceau, leur signature sur les documents à caractère décisionnel, comme l'octroi du droit de cité - disent se borner à suivre les recommandations émises par les personnes responsables de construire les dossiers, de façonner les documents, de rédiger les rapports. Outre les communes, c'est aussi ainsi que la commission cantonale chargée de l'examen préalable des dossiers de naturalisation décrit son rôle: "Les cas qui nous arrivent en commission sont très rarement discutables. [...] On est rendus attentifs à certains cas particuliers sur lesquels on se prononce. [...] Et, oui, ce n'est qu'en cas de désaccord qu'on discute» (M. Corentin, avril 2015). On retrouve l'importance de la discussion comme acte décisionnel: la commission "discute», mais ne le fait que sur les cas "discutables». Il y a donc, de manière tacite et implicite, une première évaluation qui permet de différencier ces dossiers de ceux qui ne posent pas de problèmes. 
Le caractère "discutable» de certains dossiers est relevé dans le rapport produit en amont par le Service de la justice. Un document de synthèse, qui indique pour chaque dossier s'il est «litigieux» ou "plutôt favorable», est envoyé aux membres de la commission cantonale. Il est rédigé une fois que la commune a octroyé (ou refusé) le droit de cité: "Moi, je lis tous ces dossiers en dernier. Après avoir fait ce rapport, je lis tous ces dossiers et je dis à la commission [cantonale], "Mesdames et Messieurs, là il n'y a pas de problème, là, il y a quelque chose à voir. Sur ce dossier-là, il y a des impôts qui ne sont pas payés. Ce dossier-là, il y a un problème de langue, ce dossier-là, c'est un problème". Voilà. Et dans les 98 cas [traités lors de la dernière séance], on avait juste un seul dossier où il y avait un problème d'impôts, tous les autres, c'étaient des cas où il n'y avait rien de problématique, rien à soulever, aucune discussion à avoir, donc je propose [...] de rendre un préavis favorable, et puis ils me font confiance sur ce point-là, sur mon travail» (Mme Justine, avril 2015).

Cette «confiance» est l'une des conditions qui permet aux petites mains de l'administration d'influencer la trajectoire. Ainsi, M. Costin relève que:

[Prendre la décision], ça appartient à l'organe de décision, mais nous aussi, par exemple la personne nous pose la question, donc on pense en tout cas quand la personne ne peut pas s'exprimer nous pouvons quand même dire: "Voilà cette personne a tel âge, elle a fait des efforts, bon maintenant il faut décider est-ce qu' elle est éligible ou pas.» (M. Costin, septembre 2014)

De la même manière, $M^{\text {me }}$ Justine évoque la nécessité d'examiner la "volonté» de la requérante ou du requérant à remplir les critères attendus: "On regarde quand même est-ce qu'il a eu la volonté de le faire, et puis, est-ce qu'il n'a pas pu ou est-ce qu'il n'a pas eu du tout de volonté» (décembre 2014). Plusieurs collaboratrices et collaborateurs font ainsi part de leur influence dans l'évaluation d'un dossier en mentionnant une "capacité d'appréciation" qui est "propre" à chaque collaboratrice ou collaborateur; d'autres parlent de "personnalité propre» ou encore de «sensibilité».

Tel que décrit, le système politico-administratif ne produit que très peu de décisions négatives lors de la seconde phase. La 
sélection se fait en amont sur une base purement administrative au cours de la première phase, dans laquelle aucune décision n'est prise. Pareil état de fait rend aussi difficile le contrôle des candidates et candidats sur leur dossier, qui circule entre de nombreuses instances. Au moment où la demande entame la partie décisionnelle, les cas « discutables» ont été écartés par le personnel des services cantonaux. L'influence de ce dernier dans le processus est donc capitale, alors même qu'il n'a aucun pouvoir décisionnel. La décision est présentée comme étant le fruit du "bon travail» de l'administration: "Bon, quand les dossiers arrivent à la commission, ils sont déjà bien travaillés" (M. Corentin, avril 2015). Comme montré plus haut avec l'analyse du cas de M. Charles, la standardisation des documents n'est pas sans incidence sur les prises de décision. Schématisation et automatismes produisent un effet domino dans les prises de décision ${ }^{53}$ et l'écrit autorise également des prises de pouvoir sur autrui de la part des petites mains de l'administration, des personnes dénuées de pouvoir décisionnel.

Les discussions entre pairs - au niveau communal comme cantonal - ont pour objectif d'arriver à un "consensus". C'est ce consensus qui tient lieu de décision, et même lorsque, légalement, le vote est requis, comme c'est le cas pour les Commissions communales des naturalisations et des agrégations:

En général, on n'a pas besoin de vote, on est d'accord. Moi je n'ai pas le droit de voter, je suis là en tant que membre du Conseil général, mais c'est la commission qui prend les décisions. On discute et on est souvent tous du même avis. Il peut y avoir une abstention, si on n'est pas d'accord, mais des votes, ça ne se produit quasi jamais. (M. Noël, avril 2017)

De même, au niveau du canton: «Moi, en observant [le travail de mes collègues], [...] je trouve qu'[il y a] des sensibilités différentes et alors [on arrive] toujours quand même à, enfin, à un consensus, à adopter [des points de vue] plus ou moins [semblables], une fois dans un sens, une fois dans l'autre et trouver un juste milieu» (M $\mathrm{M}^{\mathrm{me}}$ Justine, avril 2015).

53. Shaw et Kaye, 2013. 


\subsection{SYNTHÈSE: LA FRAGMENTATION DU POUVOIR DÉCISIONNEL}

La procédure de naturalisation semble fonctionner comme si elle visait à rassembler des pièces éparses qui, une fois correctement imbriquées les unes dans les autres, dévoileraient un fidèle reflet des personnes candidates à la naturalisation, et en particulier de leur intégration, ou son défaut. Notre analyse montre que cette image cohérente ne préexiste pas à la procédure; c'est cette dernière qui la produit en créant une narration spécifique. Elle ne dévoile pas "la» réalité, mais, opérant par sélection, simplification et réarticulation, imbrique les éléments perçus comme pertinents les uns avec les autres, les retaillant au passage. Ainsi, la procédure de naturalisation, et tout particulièrement l'étape cruciale que constitue l'évaluation de l'intégration, est une "opération de performation du réel " ${ }^{54}$, qui le réaménage pour le rendre conforme à la prescription. Le travail administratif est un processus de microsélection qui rassemble des documents témoignant de bribes de trajectoire des candidates et candidats, dont la "vie sociale ne cesse de s'écrire" "5 . Les "pièces justificatives» de l'administration construisent des «identités de papier ${ }^{56}$, dont la trajectoire se fait, se défait et se reconfigure continuellement au cours du temps. Le dossier administratif est un outil de médiation qui «transforme, distord, modifie le sens ou les éléments [qu'il est censé] transporter ${ }^{57}$. Cette médiation permet d'inscrire, d'organiser et de réguler ${ }^{58}$ : par la fonction d'inscription, les informations existent hors de leur contexte de production.

Bien que le travail des agents administratifs apparaisse souvent comme transparent, car représenté comme l'application objective des règles de droit, il consiste en une adaptation constante de ces règles par le biais des pratiques quotidiennes ${ }^{59}$. De même, les professionnelles et professionnels de la naturalisation négocient les pratiques par rapport aux attentes institutionnelles et politiques. Si des instructions quant au traitement des demandes sont consignées parfois très précisément, si l'intégration est définie par des conditions, elles-mêmes déclinées en critères, la complexité et la sensibilité de l'évaluation de l'intégration restent une problématique à part entière pour les collaboratrices et collaborateurs en charge des

54. Wellwe, 2013.

55. Auvert, 2008, p. 25.

56. Dardy, 1997.

57. Latour, 2006, p. 58.

58. Merzeau, 2011.

59. Weller, 2003. 
dossiers. La façon d'appliquer la loi, les directives, est une question qui anime les professionnelles et professionnels de la procédure de naturalisation interviewés dans le cadre de la recherche. Les doutes émis, leur propre conception de l'intégration - qui peut être plus ou moins conforme à ce que la loi énonce - influencent leurs pratiques.

Le personnel administratif ainsi que les élues et les élus ont des attentes face aux personnes candidates et à l'attitude qu'ils doivent adopter. Les «efforts" et la "volonté» de ces derniers pour remplir les critères attendus sont des facteurs d'évaluation non négligeables. Ils contribuent à influencer de manière directe la suite de la trajectoire et la prise de décision finale. Ces attentes façonnent la désirabilité des candidates et des candidats: se montrer désirable, ce n'est pas seulement être capable d'apporter les preuves d'une intégration consolidée, c'est adopter l'attitude attendue face à la procédure, faite d'humilité, de patience et d'acceptation.

Au total, le processus est un espace de tensions et de négociation de sens entre les personnes candidates, le personnel administratif du canton, de la Confédération et des communes, et les élues et élus communaux et cantonaux. Ces tensions sont générées par le fait qu'il faut constamment faire coïncider les cas particuliers et un cadre légal généralisant. Responsables du dossier, les employées et employés sont pris entre divers impératifs: celui de travailler conformément aux prescriptions de leur corps professionnel, celui de répondre aux attentes des candidates et candidats à la naturalisation, et celui de leur positionnement dans des administrations organisées selon des principes hiérarchiques. Ces impératifs discordants s'actualisent dans le hiatus qu'il y a entre ce que fait le traitement administratif et ce qu'il prétend faire.

L'interprétation opère à travers la sélection, mais aussi à travers la (re)formulation des informations. C'est à ce niveau que les collaboratrices et collaborateurs de la naturalisation ont une influence majeure. Distincte du pouvoir décisionnel, cette influence est minimisée ou même tue, au profit des idées de symétrie et d'équilibre, ce que reflète la sensibilité des agentes et des agents de l'État quant à l'image plus ou moins bonne de leur professionnalisme que leur renvoient les candidates et candidats. Cependant, ce jeu de miroir ne devrait pas effacer les grandes divergences entre ce qui se joue pour les uns et les autres et l'inégalité des outils à disposition des personnes candidates et des membres de l'administration. 
Le personnel de l'État peut se convaincre que sa bonne volonté est suffisante pour légitimer les choix, qu'il peut toujours les expliquer, alors que les candidates et candidats doivent sans cesse prouver leur désirabilité, y compris par l'acceptation, sans discuter et sans argumenter, des décisions qui leur sont incompréhensibles et de celles qui sont contraires à leurs intérêts.

Il reste finalement un élément important à souligner: l'analyse dissèque de manière approfondie ce qui se passe dans les services concernés, elle met en évidence les mécanismes de décision et les processus de cascade qui interviennent, où chaque instance se base sur ce que les précédentes ont rassemblé, sélectionné, synthétisé. Ces processus produisent des preuves matérielles, cisèlent des pièces d'un puzzle en construction qui, au final, ne reflète pas une image préexistante, l'intégration réelle ou véritable des personnes candidates, entendue comme une réalité donnée, observable, mais qui, au contraire, la produit, la crée. Pourtant, ce système fonctionne, et malgré l'accent mis sur la marge de manœuvre, la fausse symétrie construite entre le pouvoir des candidates et des candidats et celui du personnel administratif, il fonctionne aussi dans un sens favorable aux demandes de naturalisation. La souplesse, la malléabilité de la procédure peuvent être bénéfiques: "Donc, il y a cette souplesse aussi, hein! Un vide de procédure ou bien un flou de procédure ne sont pas utilisés forcément contre les gens!» ( $\mathrm{M}^{\mathrm{me}}$ Corinne, juin 2014). 
PARTIE IV

CONCLUSIONS 



\section{FABRIQUER L'INTÉGRATION}

TANIA ZITTOUN, FLORA DI DONATO, ÉLODIE GARROS, ANNE LAVANCHY ET PASCAL MAHON

$\mathrm{L}$

a double lecture que nous avons proposée tout au long de ce livre visait à reconstituer, d'une part, la trajectoire verticale qui dans la loi à sa déclinaison dans la jurisprudence et sa mise en pratique, et, d'autre part, la trajectoire plus horizontale de demandes de naturalisation particulières, ce qui nous a amenées à distinguer la trajectoire administrativo-légale des dossiers de la trajectoire de vie des personnes. Ce faisant, nous avons cherché à comprendre en quoi consistait «l'intégration réussie» de personnes, qui leur permet d'accéder à la nationalité suisse. Dans ce qui suit, nous retraçons d'abord les grands mouvements de cette analyse, avant de revenir aux hypothèses formulées en ouverture de cet ouvrage. Tout cela nous conduit à montrer la grande variation des compréhensions du terme intégration, qui different selon les actrices et acteurs et les contextes : la notion peut valider un état de fait, elle peut traduire la volonté d'initier un processus, ou même en saluer l'aboutissement; parfois, son usage peut avoir des effets indirects et paradoxaux. Nous indiquerons enfin en quoi notre propos pourrait avoir des implications théoriques plus larges, en esquissant deux suggestions pour la pratique.

\section{L'INTÉGRATION, DE LA LOI À LA PRATIQUE}

Notre étude interdisciplinaire de la loi nous a permis de retracer son histoire, les modalités pratiques et matérielles de son application, et les effets que celle-ci peut avoir pour la vie des personnes. Nous avons pour cela travaillé en deux grands mouvements, après l'introduction théorique et méthodologique (partie I). 
Dans un premier temps, nous avons reconstruit le contexte institutionnel de la loi sur la nationalité et de la loi sur les étrangers (partie II). Nous avons ainsi montré comment la notion d'intégration est progressivement apparue dans l'ordre juridique suisse et s'est vu attribuer des significations changeantes. Au cours du XX siècle, nous avons identifié une oscillation entre ouverture et fermeture, traduite tantôt par l'octroi de permis de séjour temporaires dans le souci de garder à distance des personnes étrangères, dans les années 1940, tantôt par l'adoption d'une loi sur l'acquisition et la perte de la nationalité, dans les années 1950. À d'autres époques, cette oscillation a amené la Suisse à utiliser l'octroi de la nationalité comme moyen d'absorber la population étrangère, comme dans les années 1920, ou encore comme mécanisme actif d'intégration dans les années 1970.

L'intégration a ensuite été définie par la loi fédérale sur les étrangers comme processus mutuel et réciproque d'adaptation entre pays d'accueil et personnes requérantes. Cette définition s'est concrètement traduite dans l'exigence adressée aux étrangères et étrangers de se familiariser avec la société et le mode de vie suisses et d'apprendre une langue nationale. Mises en application, ces exigences sont devenues des critères d'évaluation, permettant d'identifier dans quelle mesure les personnes et leurs modes de vie sont "conformes" à des attentes des autorités en grande partie implicites. De moyen d'inclusion et d'intégration, la naturalisation est donc devenue quelque chose qui se mérite et vient couronner une intégration suffisante, ou même une "intégration réussie»; le principe d'octroi de la naturalisation est passé du statut de facilitateur d'intégration à une procédure d'évaluation d'une intégration supposée déjà achevée (chapitre 3).

Cette logique est particulièrement mise en évidence par le nouveau critère d'une «intégration réussie», précisément, introduit dès 2018 comme condition d'octroi de la nationalité; l'autorité doit donc, de fait, mettre en place un système permettant d'évaluer dans quelle mesure une intégration satisfait les conditions de cette «réussite». Les autorités politiques et juridiques ont ainsi établi et précisé des critères en se fondant sur ceux qui étaient déjà utilisés dans la jurisprudence et dans la pratique et que nous avons analysés dans les chapitres 4 et 5 , à savoir les critères linguistiques, associatifs et culturels, ainsi que le critère de l'absence de mise en danger de la sûreté intérieure et extérieure et ceux de l'autonomie 
financière et de la participation à la vie économique. Dans leur application administrative, ces critères restent suffisamment flous pour laisser une marge d'appréciation importante aux cantons et aux communes. Il en résulte que l'évaluation d'une intégration "réussie» revient finalement aux actrices et aux acteurs en charge de constituer le dossier, qui sont pris entre des impératifs politiques fédéraux, des traditions cantonales plus ou moins libérales, leur «bon sens» et les enjeux relationnels des face-à-face avec les personnes requérantes (chapitre 8).

Nous avons par ailleurs illustré la manière dont cette loi était mise en pratique, en identifiant tous les acteurs et actrices impliqués, et en reconstituant la trajectoire type des dossiers de naturalisation, depuis le moment du dépôt d'une demande écrite jusqu'à une décision finale. Le schéma que nous avons proposé nous a permis de montrer que, derrière la répartition officielle en deux phases $\mathrm{du}$ traitement des dossiers (demande de l'autorisation fédérale préalable, puis octroi des droits de cité), les niveaux communal, cantonal et fédéral s'interpénètrent constamment. En particulier, nous avons mis en relief l'importance du travail préalable effectué au niveau communal et cantonal dans l'élaboration d'un préavis lors de la première phase, préavis qui détermine pour ainsi dire la suite du traitement de la demande (chapitre 5).

Dans un deuxième temps, nous avons analysé les conséquences de cet arrangement légal et institutionnel sur les trajectoires des personnes et de leurs demandes, et sur la constitution du dossier (partie III). En nous fondant sur l'étude approfondie d'une dizaine de cas, nous avons montré comment les procédures de demande de nationalité et d'évaluation de l'intégration pouvaient être vécues par les candidates et candidats, et comment ces procédures pouvaient affecter des interactions quotidiennes et des projets de vie. Dans certains cas difficiles, les personnes candidates ont fait l'expérience d'une progressive remise en question de leur intégration du fait même de la procédure (chapitre 6). Nous avons alors aussi pu mettre en évidence que celles-ci n'étaient pas passives face à ce traitement administratif et légal, et qu'elles pouvaient au contraire faire preuve d'agentivité légale, démontrant par la pratique leur engagement et leur maîtrise du système juridique suisse - pas toujours à leur avantage (chapitre 7). Enfin, en nous centrant sur le travail des actrices et des acteurs de l'administration, nous avons observé comment le traitement matériel des dossiers de demande 
de naturalisation - du passage de l'oral à l'écrit, de l'ajout de post-it aux séances de décisions - participait de la décision finale d'octroi ou de rejet. Nous avons finalement montré que le traitement administratif est une forme de prise de décision dans laquelle le pouvoir, aussi fragmenté que la trajectoire du dossier, réside finalement non pas seulement du côté des personnes qui en sont officiellement investies, mais de celui des "petites mains" de l'administration, dont le travail d'organisation et de production du dossier dicte largement l'issue de la procédure (chapitre 8).

Autrement dit, en nous intéressant aux pratiques de l'évaluation de l'intégration, nous avons mis au jour des expériences humaines et des actions complexes: des informations sont collectées, sélectionnées ou ignorées, privilégiées ou laissées dans l'ombre, transmises de personne à personne et de service à service, et progressivement transformées, concourant ainsi à la "fabrique» de l'intégration. Par ce terme de "fabrique», nous voulons mettre en évidence combien l'intégration résulte d'un long processus, distribué et médiatisé. Nous jouons tant sur l'image de la "fabrique» pour désigner le contenant - un cadre institutionnel solide, poli par l'histoire et une tradition - que sur celle des pratiques qu' elle implique - des petites mains actives tout au long d'une chaîne de montage ou de courroie de transmission, qui doivent suivre des règles, tout en comptant sur leur expérience et leur art de la bricole. Enfin, cette fabrique engage et affecte toutes les personnes concernées, qu'elles soient en charge des dossiers, ou qu'elles les aient déposés. Nous allons maintenant détailler les composantes de cette fabrique.

\section{RETOUR SUR SEPT HYPOTHÈSES}

Au terme de notre analyse, nous pouvons revenir sur les hypothèses exploratoires qui ont guidé notre travail (chapitre 1) et, sur cette base, formuler autant de propositions théoriques.

Notre première hypothèse était que les procédures de naturalisation, loin d'être de simples démarches de validation de la légitimité d'une demande, représentent des procédures de transformation de la personne. Nous avons pu en effet voir différentes modalités de modification de la personne transparaître des lois et des pratiques: au niveau légal, par exemple, l'exigence historique d'assimilation des étrangères et des étrangers par la nationalité avait bien pour but de transmuer leur étrangeté en quelque chose de plus "suisse». 
Au fil du temps, les formulations successives des exigences d'intégration visent également à transformer les personnes. En effet, bien que le législateur envisage un ajustement mutuel entre le pays hôte et les personnes migrantes, volonté mise en pratique par des initiatives cantonales (chapitres 3 et 4), l'application de la loi et, en particulier, la mise en œuvre du critère d'intégration réussie demande de fait souvent une mise en conformité de la personne avec une série d'attentes, et la capacité de prouver cette conformité. Ces attentes incluent la langue parlée, les connaissances culturelles et la participation à la vie sociale et économique (chapitre 4), mais aussi la satisfaction d'exigences plus implicites, mais reflétant des valeurs supposées requises: la patience, le goût du travail et le respect de la procédure, notamment (chapitre 8). Si ces derniers points ne sont pas explicites dans les lois ou la jurisprudence, ils reflètent les pratiques effectives, et tous suggèrent une attente globale de l'appareil juridico-administratif: la personne "intégrée " est devenue pour ainsi dire «suisse». Cela s'accompagne, de la part des personnes en charge de l'évaluation des demandes, d'une logique de suspicion et de recherche d'indices de non-intégration (chapitre 8). Ainsi, nos observations suggèrent que, s'il est clair que le but de l'octroi de la nationalité n'est plus de faciliter l'intégration, celui-ci ne vise pas non plus à cautionner l'intégration réussie par ailleurs; en fait, c'est la procédure elle-même qui participe à la production de l'intégration et donc à la transformation des personnes - minimalement parfois, mais de manière beaucoup plus profonde lorsque les trajectoires de vie des personnes sont atypiques et marquées durablement par un parcours migratoire difficile et un parcours administratif complexe dans le pays d'accueil. Nous avons donc observé un double déplacement: la nationalité cautionne plus qu'elle ne permet l'intégration; mais c'est surtout la procédure qui fabrique l'intégration qu'elle vise à évaluer.

Ce sont les logiques qui sous-tendent cette transformation que nous avons voulu examiner avec notre deuxième hypothèse exploratoire, suggérant que l'application de la loi visant à vérifier l'intégration d'une personne se traduit par une pratique dont les objectifs sont nombreux et parfois contradictoires et dont la mise en œuvre se noue dans un jeu de déterminations multiples et complexes. Nous avons ainsi vu que les lois ont évolué en fonction de la situation économique et politique de la Suisse, des liens avec les pays limitrophes et parfois de la situation géopolitique 
mondiale, et qu'elles ont été modulées par les majorités politiques au pouvoir. Accepter les étrangères et les étrangers, ou restreindre leur entrée dans le pays, peut ainsi répondre à des idéaux humanitaires, à des besoins supposés de l'économie et de la formation, ou encore à des logiques électorales (chapitre 3). La jurisprudence et les applications cantonales et communales suivent des logiques comparables à leur échelle, avec de réelles accélérations de procédure aux moments de changements liés à des élections communales ou cantonales ou de changements de responsables de service (chapitres 4, 5 et 8). Du côté des personnes employées par les services cantonaux, nous avons pu observer combien les décisions sont prises entre des volontés d'équité et de décisions équilibrées et parfois des logiques intuitives et des besoins d'efficacité (chapitres 5 et 8). L'étendue et la complexité des éléments entrant en ligne de compte dans les décisions amènent les actrices et les acteurs institutionnels à définir des procédures plus ou moins standardisées. Celles-ci sont parfois issues d'habitudes qui se généralisent; elles découlent d'autres fois d'interprétations raisonnées de la loi, ou encore stabilisent des solutions identifiées en réponse à l'analyse de problèmes. L'institution est ainsi ellemême en mouvement, que ce soit en termes de compréhension de ce que devrait être l'intégration ou en termes organisationnels, comme nous l'avons vu dans le cas de Neuchâtel, où le rapport d'intégration avait d'abord été délégué à la police, avant d'être confié au Service de la cohésion multiculturelle. Notre lecture le montre, aucune décision n'est neutre. Certes, la personne dont l'intégration est évaluée n'apparaît plus comme une personne potentiellement coupable de délit, comme c'était le cas dans les rapports de police. Il n'en demeure pas moins que confier l'évaluation de l'intégration au service en charge de faciliter la cohésion transforme aussi la mission de ce dernier, qui de fait se trouve impliqué dans la décision finale d'octroi, alors même qu'il s'en défend et que cette mission n'est pas dans sa vocation (chapitre 8). De leur côté, les personnes requérantes jouent aussi un rôle dans cette détermination, selon qu'elles sont peu réactives ou au contraire très ou trop actives, capables de donner les informations requises par les services en charge d'évaluer leur intégration, aux bons moments, ou alors qu'elles sont plus hésitantes ou revendicatives, avec une agentivité légale plus ou moins adéquate et efficace (chapitres 6 et 7). Toutes ces conduites 
sont à leur tour interprétées par le personnel administratif et par les commissions en charge de la naturalisation, à l'aune de leurs compétences respectives (chapitre 8).

Impliquant de nombreux agentes et agents de l'administration distribués spatialement et géographiquement, les décisions sont en grande partie portées par les documents matériels qui circulent entre eux. Notre troisième hypothèse exploratoire concernait le travail de l'administration en tant qu'entreprise de production de sens et de légitimité, qui se traduit notamment par la fabrication de traces lexicales et matérielles. En suivant les trajectoires des dossiers, nous avons montré combien ceux-ci - partiellement ou dans leur totalité - jouent à la fois le rôle de point de jonction entre les services et de médiateurs des décisions. En effet, les dossiers, qui résultent de l'assemblage de divers documents, officiels et internes, émis par différents agents, et de procédures de traduction et de transformation, deviennent l'avatar de la personne qu'ils sont censés représenter; les traces qui le composent ont toutes des effets de sens qui dépassent et débordent cette personne. En fin de compte, c'est au dossier que les instances investies officiellement d'un pouvoir décisionnel réagissent, et c'est lui, plus que la personne qu'il concerne, qui est évalué et qui, en conséquence, devient porteur d'une décision que le pouvoir ne semble qu'avaliser (chapitres 5 et 8 ).

Symétriquement, nous nous sommes intéressées aux trajectoires des personnes; notre quatrième hypothèse exploratoire nous invitait à examiner la demande de naturalisation non pas comme un simple acte administratif, mais comme l'expression d'un projet de la personne, traduisant une ou des imaginations de soi, et susceptible d'avoir dans ce sens des implications affectives et identitaires pour elle et son entourage dans différentes sphères d'expérience. Une analyse transversale des cas nous a permis de constater que la plupart des demandes de naturalisation exprimaient en effet une volonté de voir validée ou socialement reconnue l'expérience d'avoir vécu une part significative de sa vie en Suisse. Trouvant souvent racine dans une lecture de la trajectoire passée et des événements qui ont permis de se sentir "chez soi» dans le pays, les demandes traduisent des projets à long terme, incluant une imagination de sa vie future, de sa famille ou d'activités professionnelles à venir. Ainsi, la demande de naturalisation devient en partie la possibilité de faire reconnaître comme légitime l'expérience subjective et parfois 
partagée par l'entourage d'être "intégré·e» en Suisse. S'il est vrai que cette demande est quelquefois liée à la recherche d'avantages pratiques (liberté de circulation, facilités professionnelles, regroupement familial), elle a une grande portée symbolique, car elle implique aussi une reconnaissance de légitimité et d'appartenance à la communauté (chapitre 6).

La cinquième de nos hypothèses exploratoires nous invitait dès lors à examiner les conséquences, pour la personne, du passage par une procédure de naturalisation; en particulier, nous nous demandions si, et dans quelle mesure, la trajectoire de vie de la personne pouvait être transformée par la trajectoire administrative. Nous avons montré que les aléas de la procédure - demandes de compléments d'information, identifications de problèmes, délais, etc. - créent un effet de surprise, et remettent très souvent rapidement en question le sentiment de légitimité qui accompagne la démarche de naturalisation (chapitres 6 et 7). Par ailleurs, les méandres de la procédure peuvent rendre l'administration difficile à cerner pour les personnes candidates, et ses demandes et ses décisions leur paraissent souvent opaques; enfin, la temporalité de la procédure peut créer des décalages avec les aléas des trajectoires de vie, qui deviennent sources d'angoisse et d'incertitude. Il en résulte que les démarches administrativo-légales affectent les trajectoires de vie. C'est ainsi qu'en cas d'acceptation, même après des doutes, la personne bénéficie des perspectives nouvelles, pratiques et symboliques, conférées par la nationalité. En revanche, en cas de refus, souvent même après une longue procédure, les personnes sont amenées à questionner leur image parfois idéalisée de la Suisse et de son fonctionnement administratif et légal. Le refus a aussi des conséquences dans différentes sphères d'expérience. Au niveau symbolique, certaines personnes en sont venues à se voir comme «moins» intégrées qu'elles ne pensaient l'être ou même qu'elles ne l'étaient. D'autres se sentent plus radicalement remises en question, internalisant le «doute» qu'elles perçoivent à leur égard. Plus concrètement, les aléas et rebondissements de la procédure administrative peuvent mener les personnes à devoir changer d'emploi, à réorganiser leurs dépenses, ou à rendre inatteignables certains futurs imaginés, voire à les déstabiliser plus durablement. Dans tous les cas, il est indéniable que la procédure de naturalisation transforme les trajectoires de vie, qu'elle facilite l'intégration ou, au contraire, en restreigne la possibilité (chapitre 6). 
Néanmoins, et c'était là l'objet de notre sixième hypothèse exploratoire, les personnes ne sont pas passives dans leur rapport à la loi et aux administrations; elles peuvent faire preuve d'agentivité légale - d'un pouvoir de comprendre et d'agir face à la loi. Dans les cas examinés, les personnes requérantes tendent à se positionner de manière collaborative, subversive ou contestataire face à la loi. Les positionnements initiaux évoluent au fur et à mesure de la compréhension du système et de l'expérience de la procédure, et sont souvent changés après les réponses des services, lorsque ceux-ci remettent en question le bien-fondé de la demande. Quand l'opacité et la temporalité de la trajectoire administrative ne découragent pas complètement les personnes candidates, notre analyse montre que celles-ci peuvent alors développer une agentivité légale plus ou moins efficace au fil de la procédure. Cette agentivité se traduit notamment par une connaissance des lois, la maîtrise progressive d'un style communicationnel plus formel, passant de lettres manuscrites et personnelles à des courriers dactylographiés et usant d'un vocabulaire technique correspondant davantage à ce que peuvent être les attentes formelles. Toutefois, cette maîtrise ne génère pas toujours une réponse positive. Pour des raisons liées à la temporalité et à la fragmentation des prises de décisions, une réponse pourtant pertinente n'arrive pas au bon moment du point de vue de l'administration. Nous avons ainsi retracé des cas dans lesquels des arguments visant à faire la preuve de l'intégration de la personne dans sa vie quotidienne ne sont pas forcément retenus. Dans d'autres cas, ce sont d'autres éléments qui entravent la réussite du projet, comme la difficulté d'apprendre la langue locale ou celle d'accéder à un travail qualifié, ou encore la volonté de garder ses coutumes, notamment vestimentaires, vis-à-vis de la Commission des naturalisations. En outre, le manque de connaissances et de formation spécifiques des avocates et avocats appelés à intervenir ne facilite pas toujours la réussite du projet (chapitre 7).

Il découle de ces points que la procédure visant à vérifier l'intégration de personnes candidates à la naturalisation se déroule au travers d'un dialogue parfois inégal avec les services administratifs et juridiques. Notre septième hypothèse nous a invitées à examiner précisément les transactions qui se nouent entre trajectoire administrative et trajectoire de vie et, en particulier, la manière dont les personnes candidates, mais aussi les actrices et les acteurs de l'administration, cherchent mutuellement à se comprendre, ou rendent 
invisibles certaines de leurs actions. D'une part, nous avons pu observer que les personnes requérantes sont parfois surprises d'apprendre que l'administration connaît des faits les concernant qu'elles-mêmes ignorent ou avaient oublié, comme une vieille facture impayée; parfois aussi, elles souhaitent dissimuler certains faits; ou encore, elles ont l'impression que leurs interlocutrices et interlocuteurs de l'administration se cachent derrière les règles et leurs rôles (chapitres 6 et 7). D'autre part, lorsque nous sommes entrées dans le détail du traitement des dossiers, nous avons vu que les personnes en charge des dossiers sont occupées à décomposer des récits et des trajectoires complexes en écritures, pièces et documents qui peuvent être passés de main en main, et qui à leur tour créent un récit sur les trajectoires de vie, qui oriente fortement l'analyse qui en sera faite. Il est aussi apparu que dans leur recherche de la "bonne volonté» des personnes requérantes, les professionnelles et les professionnels évaluent leur "honnêteté», quand bien même nous avons par ailleurs suggéré que les personnes ont intérêt à formuler leur demande dans des formes correspondant aux attentes. Nous avons enfin montré que, de cette manière, les professionnelles et les professionnels rendaient leur propre activité d'une certaine manière invisible, laissant les Conseils communaux prononcer la décision, alors même que la façon dont les dossiers sont manufacturés préjuge du résultat de leur évaluation (chapitre 8). Finalement, il faut rappeler que la procédure comprend deux phases. La première, visant l'autorisation (fédérale) préalable de naturalisation, opère une fonction de tri dont le résultat est que seules les demandes qui ont de bonnes chances d'aboutir atteignent la deuxième phase. Cette fonction de tri, qui résulte probablement d'une volonté d'efficacité, se traduit en fin de compte par un tour de passe-passe où les dossiers «difficiles» sont escamotés (chapitre 5).

Il résulte de notre analyse que la procédure de naturalisation reposant sur une évaluation de l'intégration des personnes crée des effets complexes. En tant qu'appareil institutionnel, elle opère comme une machinerie qui participe à la réinvention et de la transformation des personnes. Elle ne laisse aucune personne indemne: les personnes candidates dont les demandes sont refusées se retrouvent parfois remises en question, voire fragilisées ou même "désintégrées»; celles dont les demandes sont acceptées ont été transformées par la procédure et évidemment par ses débouchés; et 
finalement, celles qui travaillent dans les bureaux vivent parfois des conflits éthiques; elles doivent toutes apprendre à transformer des destins uniques, et qui parfois les touchent, en dossiers qui peuvent être questionnés, réorganisés, manipulés et transmis. Ainsi avonsnous montré que la mise en application de la loi sur la nationalité devient une gigantesque fabrique de l'intégration.

\section{D'UNE ÉTUDE DE CAS À LA RECHERCHE DE PROPOSITIONS GÉNÉRALES}

Avant de clore cet ouvrage, nous souhaitons mettre en évidence certaines implications de notre lecture des procédures de naturalisation. Nous suggérons pour ce faire que, bien que notre investigation soit limitée à un seul contexte, elle est néanmoins susceptible d'avoir des implications plus générales.

Nous avons indiqué à plusieurs reprises les spécificités de cette analyse de la fabrique de l'intégration en Suisse, que nous avons ancrée dans les lois et les pratiques du Canton de Neuchâtel, sur la base d'une loi fédérale qui a entre-temps été revue. Ainsi que nous l'avons signalé, notre étude est fondée sur l'analyse détaillée des cas d'une dizaine de demandes qui ont occasionné des procédures longues et compliquées, et qui ne reflètent pas la grande majorité des demandes de naturalisation, qui sont traitées facilement et débouchent sur des décisions positives. Dans quelle mesure pouvons-nous, dans ces conditions, généraliser à partir de cette étude de cas, et de cas limites?

L'étude approfondie d'un cas particulier a l'avantage de mettre en évidence des processus complexes de portée générale. D’une part, les trois niveaux d'analyse que nous avons distingués - la trajectoire administrativo-légale, la trajectoire de vie, les transactions - sont plus généralement valides lorsqu'il s'agit d'analyser des trajectoires administratives et légales. D'autre part, l'examen de cas extrêmes ou spécialement compliqués que nous avons proposé met en lumière les fonctionnements, mais aussi les faiblesses d'un système complexe, et en particulier la question de la distribution ou de la dilution des responsabilités décisionnelles. Nous pouvons donc penser que les sept propositions auxquelles nous ont amenées nos hypothèses sont susceptibles d'éclairer de nombreuses pratiques administratives. Premièrement, les procédures légales visant à l'évaluation de personnes participent à leur transformation. Deuxièmement, des procédures administratives et légales 
complexes, impliquant de nombreux actrices et acteurs sur une longue durée, sont génératrices de tensions et de contradictions. Troisièmement, les supports matériels par lesquels se font ces procédures et les processus de traduction qu'ils impliquent participent à la transformation des significations. Nous avons, quatrièmement, mis en évidence combien les démarches administratives engagent plus globalement les trajectoires de vie des personnes - leur développement, leur imagination et leur besoin de reconnaissance -, et que les décalages entre la temporalité administrative et le temps vécu pouvaient s'avérer difficiles à vivre, voire, cinquièmement, réellement transformer les trajectoires de vie; nous pensons que cela éclaire de nombreuses situations dans lesquelles les personnes se trouvent confrontées à des institutions complexes. En sixième lieu, nous avons constaté l'importance de l'agentivité des personnes face à ces institutions - ses conditions de possibilité et ses conséquences possibles. Enfin, en distinguant trajectoire administrative et légale et trajectoire de vie, nous avons pu faire un septième constat, lié aux transactions et tensions complexes qui se nouent et se dénouent: celles-ci ont des conséquences tant pour les représentantes et les professionnels de l'administration que pour les personnes qui font appel à leurs services; nul n'est à l'abri du travail de transformation qu'implique une telle fabrique. Là aussi, nous pensons pouvoir inviter à l'examen de processus comparables dans une large palette de situations équivalentes, contribuant ainsi à la compréhension de la loi en action.

Enfin, nous voulons encore une fois signaler qu'une telle analyse et les propositions que nous avons faites n'ont été possibles que parce que nous avons, tout au long de notre réflexion et de notre étude, croisé et coordonné les regards de l'analyse juridique et de la sociologie du droit, de l'anthropologie des institutions et de la psychologie socioculturelle. Là encore, un temps long a été la condition indispensable à notre travail. Nous en tirons deux observations. La première concerne la pratique et la politique de la recherche sur des réalités sociopolitiques complexes: il nous semble que le temps et la multiplicité des regards sont des conditions nécessaires à l'identification de principes généraux émergeant d'une analyse d'une grande complexité, comme les enjeux sociaux et politiques de l'évaluation du corpus juridique sur des questions sensibles. L'autre concerne l'implication des chercheuses et des chercheurs qui en découle: une telle trajectoire de recherche, dans la durée, 
sur des processus complexes, socialement et humainement chargés, prise dans le faisceau de nos regards croisés, n’a assurément laissé aucune d'entre nous indemne.

\section{OUVERTURES ET IMPLICATIONS POUR LA PRATIQUE}

Nos analyses éclairent un certain nombre de points sensibles, des tensions qui rendent inutilement difficiles les trajectoires de naturalisation, pour les personnes candidates comme pour les personnes qui travaillent dans les administrations. Bien qu'il ne soit pas strictement de notre ressort de faire des recommandations, nous voulons ici indiquer deux suggestions et une piste de réflexion, qui toutes pourraient avoir des implications pour la pratique.

Premièrement, il nous semblerait pertinent de penser à la mise en place de formations visant à l'explicitation des objectifs et de la nature des procédures de naturalisation à l'usage des personnes candidates qui le souhaiteraient. Des formations dispensées par les responsables de l'évaluation des demandes ou par des personnes en charge de dossiers amèneraient ces personnes à devoir expliciter des implicites et des procédures considérées comme allant de soi. Cela aurait le double avantage de clarifier, pour les personnes candidates, ce qui leur est demandé, mais aussi la signification des étapes de la procédure et des critères de décision, réduisant ainsi l'opacité de la procédure et l'inquiétude qu'elle génère. Du côté de l'administration, cela permettrait de préciser les attentes, voire de les simplifier et de les homogénéiser.

Deuxièmement, nous pensons qu'il serait utile de réfléchir à une simplification des temporalités dans la procédure de naturalisation. Notre analyse montre que la complexification et la démultiplication en plusieurs phases et étapes qui se chevauchent et s'entremêlent la rendent opaque. Elles risquent aussi d'induire une déshumanisation d'une procédure qui, paradoxalement, a pour but d'amener des personnes spécifiques à rejoindre une communauté. Ainsi, les personnes qui connaissent bien les dossiers ne voient pas les personnes candidates; les personnes qui rencontrent les candidates n'ont pas de réel pouvoir décisionnel (ou pas officiellement); et, finalement, les Commissions des naturalisations et des agrégations, qui prennent les décisions d'octroi ou de refus du droit de cité communal, fondent en général leur appréciation sur l'entretien avec les candidates et candidats, après avoir pris connaissance des 
dossiers qu'elles tendent à considérer comme des artefacts administratifs «désincarnés», voire parfois "mal ficelés».

Ce dernier point nous amène à suggérer une piste de réflexion novatrice. Nous avons en effet pu mettre en évidence, d'une part, que la procédure reste, pratiquement pour toutes les personnes qui y sont impliquées, d'une certaine opacité, si ce n'est d'une opacité certaine, et qu'elle se caractérise, au moins dans les cas dits "problématiques", par une temporalité longue, voire très longue, confinant parfois à des durées qui paraissent difficilement justifiables dans un État de droit. Nous avons montré aussi, d'autre part, que le système mis en place implique une (très) forte fragmentation, pour ne pas dire une dilution, des responsabilités: celles et ceux qui gèrent et «fabriquent» les dossiers ne voient pas nécessairement les personnes candidates et n'ont pas de pouvoir de décision, quand bien même leur rôle - ou celui du dossier - dans la procédure et dans la décision est souvent prépondérant; celles et ceux qui établissent les préavis ne voient pas non plus nécessairement les personnes candidates, de même, d'ailleurs, que celles et ceux qui décident formellement, etc. À cela s'ajoute que, dans chaque phase et à chaque étape de la procédure, interviennent de nouvelles actrices et de nouveaux acteurs, issus de différents services de l'administration (Service de la justice, Service de la cohésion multiculturelle, avec souvent consultation d'autres services et entités, commission tripartite chargée de l'examen préalable des demandes, etc.) et de différentes entités ou autorités politiques, qui plus est de collectivités publiques de niveau différent (Commission communale des naturalisations, Conseil communal, Conseil d'État). Et à chacune de ces étapes correspond un (nouveau) délai de traitement du dossier, ce qui a évidemment pour effet de rallonger sensiblement l'ensemble de la procédure.

Face à ces constats, nous nous permettons de poser la question - sous forme peut-être d'un pavé jeté dans la marre - de savoir si une réelle simplification et une meilleure lisibilité de la procédure ne pourraient pas être atteintes par une réforme qui inclue à la fois un recentrage des compétences et des responsabilités, une réduction des délais de traitement des demandes et une clarification de la procédure - et de leur situation - pour les personnes requérantes.

Une telle réforme pourrait par exemple consister, à notre sens, à prévoir que les demandes soient, à partir de l'ouverture 
de la procédure, traitées par une sorte de commission "mixte", composée d'une représentante ou d'un représentant de chacun des services cantonaux concernés (Service de la justice, COSM et, éventuellement, Service des migrations), mais aussi de la commune de résidence (un ou deux membres de la Commission des naturalisations, par exemple). Cette commission serait chargée soit de décider - au moins dans les cas clairs -, soit simplement ${ }^{1}$ de préaviser à l'adresse des autorités - à la fois cantonale et communale - compétentes, le Conseil communal et le Conseil d'État.

Des règles relativement précises pourraient ou devraient être adoptées pour régler la procédure, notamment afin de prévoir les modalités de traitement des demandes, par exemple la confection du rapport d'enquête et l'obligation, pour la commission "mixte", d'entendre et d'auditionner les personnes candidates. On pourrait aussi prévoir les délais de traitement des dossiers, de manière à encadrer et limiter la durée des diverses étapes de la procédure, etc. Un tel système aurait à nos yeux pour avantage de recentrer - ou de concentrer - les compétences et responsabilités, dans les mains d'une entité collégiale, formée de personnes issues de plusieurs entités et des deux niveaux de collectivités publiques et non plus d'une succession de personnes isolées. Cela aurait aussi pour effet de substituer une temporalité unique à une succession d'autorités se prononçant les unes après les autres, avec à chaque fois un nouveau délai de traitement.

Nous sommes conscientes de ce qu'une telle piste de réflexion remet en question - en les fusionnant en partie - les compétences respectives de chacune des collectivités publiques concernées (communes et canton). Toutefois, étant donné la complexité des dossiers, les implications légales des cas, les questions d'égalité de traitement et la part de subjectivité dans l'évaluation des demandes que nous avons constatées, ne serait-il pas bon de confier les décisions à des personnes formées à tous ces aspects, et assumant une responsabilité collégiale, en partageant une bonne connaissance des cas et des personnes requérantes, ainsi que des implications légales, politiques et psychologiques des décisions prises?

De même, nous avons à plusieurs reprises souligné les conséquences économiques, humaines et sociales des durées des procédures. Or, la mesure suggérée ci-dessus pourrait concourir

1. Si l'on ne veut pas déléguer des autorités politiques à un organe "administratif» la compétence de décider formellement. 
à raccourcir ces durées. Elle aurait aussi pour effet de permettre de donner aux personnes requérantes, dans les cas les plus problématiques, une réponse relativement rapide, même négative, et non de les laisser de longs mois, voire de longues années, dans l'attente d'une décision leur indiquant en fin de compte qu'elles ou ils ne remplissent pas les exigences d'intégration (ou d'une intégration "réussie", pour reprendre les termes de la nouvelle loi), ou qu'il existe des doutes sur leur intégration. Une réponse, même négative, dans des délais raisonnables, serait probablement mieux comprise des personnes candidates et aurait sans doute pour effet de leur rendre leur responsabilité et, par voie de conséquence, leur autonomie, en leur permettant de prendre les mesures propres à remédier aux "lacunes» identifiées (améliorer leurs connaissances linguistiques, éponger leurs dettes, etc.).

Nous espérons que les propositions modestes ou osées esquissées ci-dessus - formations proposées aux personnes candidates, simplification des procédures et recentrage des compétences et des responsabilités, ainsi que des délais de traitement des dossiers - pourront contribuer à une réflexion dont la finalité serait de rendre les procédures de naturalisation plus rationnelles et transparentes, de manière à permettre aussi aux personnes concernées d'aborder ces procédures de manière plus confiante et autonome ou, au moins, de mieux les comprendre. 


\section{AUTEUR·E·S}

Flora Di Donato est professeure en philosophie du droit et formation clinico-légale à l'Université Federico II de Naples (Département de droit). En utilisant une approche narrative, elle analyse le droit du point de vue des personnes concernées, qu'elles soient étrangères ou nationales.

Élodie Garros est docteure en psychologie sociale, spécialisée dans l'étude de la construction et de la transmission des savoirs. Elle est chargée de projets dans le domaine de l'ingénierie de formation et d'orientation à l'Université Paul Sabatier à Toulouse.

Anne Lavanchy est professeure à la HES-SO (Haute école de travail social, Genève), spécialisée en anthropologie politique. Ses publications portent sur les inégalités structurelles et les rapports sociaux de sexe et de race, l'éthique et la méthodologie.

Pascal Mahon est professeur de droit constitutionnel, suisse et comparé, à la Faculté de droit de l'Université de Neuchâtel. Il s'intéresse aux institutions politiques et aux droits fondamentaux de la personne, notamment dans les contextes de migration.

Tania Zittoun est professeure en psychologie socioculturelle à la Faculté des lettres et sciences humaines de l'Université de Neuchâtel. Elle étudie le développement des personnes tout au long de leur vie, dans des contextes complexes, et le rôle qu'y joue l'imagination. 



\section{BIBLIOGRAPHIE}

ABÉLÈs Marc (1995), "Pour une anthropologie des institutions", L'Homme, vol. 35, n 135, pp. 65-85.

ACHERMAnN Alberto (2017), "ad art. 4 LEtr», in Cesla Amarelle et Minh Son Nguyen (éds), Code annoté de droit des migrations, Vol. II: Loi sur les étrangers (LEtr), Berne: Stämpfli, pp. 24-44.

Achermann Alberto, Amarelle Cesla, et KurT Stefanie (2017), «ad art. 56 LEtr", in Cesla Amarelle et Minh Son Nguyen (éds), Code annoté de droit des migrations, Vol. II: Loi sur les étrangers (LEtr), Berne: Stämpfli, pp. 525-533.

Aeby Gaëlle, et BerTHOd Marc-Antoine (2011), «Entrer et sortir des institutions ", Tsantsa, n ${ }^{\circ}$ 16, pp. 6-17.

Ahearn Laura M. (2001), "Agency», in Alessandro Duranti (éd.), Key Terms in Language and Culture, Malden, MA: Blackwell, pp. 7-10.

AgIE Michel (2008), Gérer les indésirables - Des camps de réfugiés au gouvernement humanitaire, Paris: Flammarion.

AKrich Madeleine (2010), "Comment décrire les objets techniques?», Techniques \& Culture, vol. 1, n 54-55, pp. 205-219.

Alfieri Anthony A. (2016), "Rebellious Pedagogy and Practice», 23 Clinical Law Review, 5, p. 36.

Alfieri Anthony A. (1990-1991), "Reconstructive Poverty Law Practice: Learning Lessons of Client Narrative», Yale Law Journal, vol. 100, pp. 2107-2147.

Allard Olivier (2012), «Bureaucratic Anxiety. Asymmetrical interactions and the role of documents in the Orinoco Delta, Venezuela", HAU: Journal of Ethnographic Theory 2 (2), pp. 234-256. 
Althabe Gérard, et Hernandez Valeria A. (2004), «Implication et réflexivité en anthropologie», Journal des anthropologues [En ligne], pp. 98-99.

Amarelle Cesla (éd.) (2012), L'intégration des étrangers à l'épreuve du droit suisse. Fondements et applications pratiques, Berne: Stämpfli.

Amarelle Cesla, et Nguyen Minh Son (éds) (2014), Code annoté de droit des migrations. Vol. II: Loi sur la nationalité (LN), Berne: Stämpfli. Amarelle Cesla, et NGuyen Minh Son (éds) (2017), Code annoté de droit des migrations. Vol. II: Loi sur les étrangers (LEtr), Berne: Stämpfli. Amsterdam Anthony G., et Bruner Jerome (2000), Minding the Law, Cambridge: Harvard University Press.

ANDERSON Benedict (2002 [1983]), Limaginaire national. Réflexions sur l'origine et l'essor du nationalisme (édition en anglais: 1983 Imagined Communities, traduction P.-E. Dauzat), Paris: La Découverte/Poche.

Appadurai Arjun (1986), The social life of things. Commodities in a cultural perspective, Cambridge: Cambridge University Press.

ARGAST Regula (2013), "Entre tradition et innovation: le droit de cité suisse dans le nouvel État fédéral, 1848-1898", in Brigitte Studer, Gérard Arlettaz et Regula Argast (éds), Le droit d'être suisse, Acquisition, perte et retrait de la nationalité de 1848 à nos jours, Lausanne: Antipodes, pp. 45-76.

ArlettAZ Gérard (2013), "L'“assimilation”, but ultime de l'octroi de la nationalité? 1898-1933", in Brigitte Studer, Gérald Arlettaz et Regula Argast (éds), Le droit d'être suisse, Acquisition, perte et retrait de la nationalité de 1848 à nos jours, Lausanne: Antipodes, pp. 77-115.

Arlettaz Silvia, et ArletTAz Gérard (2004), La Suisse et les étrangers: immigration et formation nationale (1848-1933), Lausanne: Antipodes. AUBERT Jean-François (1958), «Le statut des étrangers en Suisse», in Revue de droit suisse, I, pp. 215-253.

AUBERT Jean-François (1967 [réédition 1991]), Traité de droit constitutionnel suisse, Neuchâtel: Éditions Ides et Calendes.

AUberT Jean-François, et MAHON Pascal (2003), Petit commentaire de la Constitution fédérale de la Confédération suisse du 18 avril 1999, Zurich/Bâle/Genève: Schulthess.

Auvert Anne-Julie (2008), «Le sociologue et les archives des enquêtés", Sociologie et sociétés, vol. 40, nº 2, pp. 15-34. 
AuYERO Javier (2011), "Patients of the state. An Ethnographic Account of Poor People's Waiting», Latin American Research Review 46 (1), pp. 5-29.

Bacigalupo Ana Mariella (2016), Thunder Shamans. Making History with Mapuche Spirits in Chile and Patagonia, Austin: University of Texas Press.

BAKHTINE Mikhail (1987), Esthétique et théorie du roman, Paris: Gallimard.

Barbier Rémi, et Trépos Jean-Yves (2007), «Humains et nonhumains: un bilan d'étape de la sociologie des collectifs", Revue d'anthropologie des connaissances, vol. 1, $\mathrm{n}^{\circ} 1$, pp. 35-58.

BARTH Frederik (éd.) (1969), Ethnic groups and boundaries: the social organization of culture difference, Bergen/Oslo/Londres: Universitetsforlaget/George Allen \& Unwin.

BARTOLI Celia (2016), "Legal clinics in Europe: for a commitment of higher education in social justice», Diritto e questioni pubbliche, numéro spécial, avril 2016.

Béal Arnaud, Kalampalikis Nikos, Fieulaine Nicolas, et HaAs Valérie (2014), «Expériences de justice et représentations sociales: l'exemple du non-recours aux droits", Les Cahiers Internationaux de Psychologie Sociale, vol. 3, n 103, pp. 549-573.

Berg Bruce L. (2004), Qualitative Research Methods for the Social Sciences, Boston: Pearson Education.

BERG Marc (1996), «Practices of reading and writing: the constitutive role of patient record in medical work", Sociology of Health \& Illness, vol. 18, n4, pp. 499-524.

Berger Peter, et LuckMann Thomas (1966), The social construction of reality: A treatise in the sociology of knowledge, Londres: Penguin.

Bigler Olivier, et Bussy Yannick (2017), "ad art. 96 LEtr», in Cesla Amarelle et Minh Son Nguyen (éds), Code annoté de droit des migrations. Vol. II: Loi sur les étrangers (LEtr), Berne: Stämpfli, pp. 1035-1051.

Binder Guyora, et Weisberg Robert (2000), Literary criticisms of law, Princeton: Princeton University Press.

Bion Wildred R. (1989), Learning from experience, Londres: Karnac Books. 
BLANDin Bernard (2002), La construction du social par les objets, Paris: PUF.

BLEE Kathleen M. (1993), «Evidence, empathy, and ethics: lessons from oral histories of the klan ", The Journal of American History 80, $\mathrm{n}^{\circ}$ 2, pp. 596-606.

BLOCK Frank S. (2008), "Access to justice and the global clinical movement", Washington University Journal of Law and Policy, vol. 28, pp. 111-139.

BLock Frank S. (éd.) (2011), The Global Clinical Movement, Oxford: Oxford University Press.

Boccagni P., et Pollini G. (2012), Lintegrazione nello studio delle migrazioni. Teorie, indicatori, ricerche, Milan: Angeli.

Boden Rebecca, Epstein Debbie, et Latimer Joanna (2009), "Accounting for Ethos or Programmes for Conduct? The Brave New World of Research Ethics Committees", The Sociological Review 57, $\mathrm{n}^{\circ}$ 4, pp. 727-749.

Bonilla Daniel (2015), «Fact-finding mission, legal clinics and the politics of legal knowledge», in Philiph Alston et Sarah Knuckey (éds), The Transformation of the Human Rights Fact-Finding, Oxford: Oxford University Press, pp. 107-128.

BorzeIX Anni, et FraEnKel Béatrice (2001), Langage et travail. Communication, cognition, action, Paris: CNRS Éditions.

Bourdieu Pierre (1979), La distinction. Critique sociale du jugement, Paris: Les Éditions de Minuit.

Bourdieu Pierre (1982), "Les rites comme actes d'institution", Actes de la recherche en sciences sociales, vol. 43, $\mathrm{n}^{\circ} 1$, pp. 58-63.

Bourdieu Pierre, et Wacquant Loïc (1992), Réponse. Pour une anthropologie réflexive, Paris: Seuil.

BRINKMANN Svend (2004), "Psychology as a moral science: Aspects of John Dewey's psychology", History of the Human Sciences, vol. 17, $\mathrm{n}^{\circ} 1$, pp. 1-28.

Brinkmann Svend (2006), "Mental life in the space of reasons", Journal for the Theory of Social Behaviour, vol. 36, $\mathrm{n}^{\circ}$ 1, pp. 1-16.

Brinkmann Svend (2010), Psychology as a Moral Science: Perspectives on Normativity, New York: Springer. 
BROOKS Peter (1992), Reading for the Plot, Cambridge (MA): Harvard University Press.

Brown Stephen D., et ReAvey Paula (2015), Vital memory and affect: Living with a difficult past, Londres: Routledge.

Bruner Jerome S. (1990), Acts of meaning, Cambridge: Harvard University Press.

BRUNER Jerome S. (1991), "The Narrative Construction of Reality", Critical Inquiry, vol. 18, $\mathrm{n}^{\circ}$ 1, pp. 1-20.

BRUNER Jerome S. (1997a), ... car la culture donne forme à l'esprit. De la révolution cognitive à la psychologie culturelle, Genève: Eshel.

BRUNER Jerome S. (1997b) «A Narrative Model of Self-Construction", in Joan Gay Snodgrass, Robert L. Thompson (éds), The Self across Psychology: Self-recognition, Self-awareness and Self-concept, New York: New York Academy of Sciences.

BRUner Jerome S. (2003), Making Stories, Cambridge (MA) : Harvard University Press.

BRUNER Jerome S. (2005), Pourquoi nous racontons-nous des histoires? Le récit au fondement de la culture et de l'identité individuelle, Paris: Pocket.

Callon Michel (2006), "Sociologie de l'acteur réseau», in Madeleine Akrich, Michel Callon et Bruno Latour (éds), Sociologie de la traduction: Textes fondateurs, Paris: Presses des Mines, pp. 267-276.

Callon Michel, et LaW John (1997), "Lirruption des non-humains dans les sciences humaines: quelques leçons tirées de la sociologie des sciences et des techniques", in Bénédicte Reynaud (éd.), Les limites de la rationalité, t. 2: Les figures du collectif, Paris: La Découverte, coll. «Recherche».

CAMPISI Laura (2014), Die rechtliche Erfassung der Integration im schweizerischen Migrationsrecht, Zwischen rechtlichen Vorgaben und innenpolitischen Realitäten, Zurich/Saint-Gall: Dike Verlag AG

Castellano Ursula (2007), "Becoming a Non-Expert and Other Strategies for Managing Fieldwork Dilemmas in the Criminal Justice System ", Journal of Contemporary Ethnography 36, n 6, pp. 704-730. Cattacin Sandro, et Bülent Kaya (2001), «Le développement des mesures d'intégration de la population migrante sur le plan local en Suisse. Enquête dans le cadre de l'étude mandatée par le PNR 39, 
L'histoire de la migration et de l'intégration en Suisse», Neuchâtel: Forum suisse pour l'étude des migrations.

CEFAï Danil (1996), "La construction des problèmes publics. Définitions de situations dans des arènes publiques ", Réseaux, vol. 14, $\mathrm{n}^{\circ} 75$, pp. 43-66.

Centurvres Pierre (1991), «Intégration et Naturalisation: l'exemple suisse», Terrain, 15, pp. 135-144.

Clémence Alain, et Doise Willem (1995), «La représentation sociale de la justice: une approche des droits dans la pensée ordinaire", L'année Sociologique, vol. 45, n 2, pp. 371-400.

CMR (2015), Étude sur la procédure de naturalisation dans le canton de Fribourg. En ligne: http://www.fr.ch/imr/files/pdf72/ CMR_Etude_naturalisation.pdf.

Cole Michael (1998), Cultural psychology: A once and future discipline, Harvard: Harvard University Press.

Comaroff Jean, et Comaroff John L. (2003), "L'échelle inconfortable de l'ethnographie. Anthropologie postcoloniale et violence de l'abstraction", Traverse 3, pp. 19-50.

Commaille Jacques (2015), À quoi nous sert le droit?, Paris: Folio.

CoOren François (2004), "Textual agency: How texts do things in organizational settings », Organization, vol. 11, pp. 373-393.

Cornish Flora, ZitToun Tania, et Gillespie Alex (2007), «A cultural psychological reflection on collaborative research. Conference Essay: ESF Exploratory Workshop on Collaborative Case Studies for a European Cultural Psychology", Forum Qualitative Sozialforschung/ Forum: Qualitative Social Research, vol. 8, n³, Art. 21. [37 paragraphes].

Corten Olivier (2013), "Le "droit en contexte" est-il incompatible avec le formalisme juridique?», Revue interdisciplinaire d'études juridiques, vol. 70, pp. 70-76.

Cover Robert, 1983-84, "The Supreme Court, 1982 - TermForeword: Nomos and Narrative", Harvard Law Review, n 97, pp. 4-68.

Cretton Viviane (2012), Serious Games. S'installer en Valais (exposition au Musée du Val de Bagnes et recherche HES-VS).

Crozier Michel, et Friedberg Erhard (1977), L'acteur et le système, Paris: Seuil. 
Crozier Michel, et Friedberg Erhard (1980), Actors and Systems. The Politics of Collective Action, Chicago: The University of Chicago Press. Crozier Michel, et Friedberg Erhard (2000 [1995]), «Organisations et action collective: notre contribution à l'analyse des organisations", in Michel Crozier (éd.), À quoi sert la sociologie des organisations?, Paris: Éditions Seli Arslan, pp. 130-160.

Crozier Michel (1961), «De la bureaucratie comme système d'organisation", European Journal of Sociology, 2 (1), pp. 18-50.

DaHinden Janine, et EFFIONAY Denise (2009), Challenges and Strategies in Empirical Fieldwork with Asylum Seekers and Migrant Sex Workers, in I. Van Liempt et V. Bigler (éds), The Ethics of Migration Research Methodology. Dealing with Vulnerable Migrants, Brighton: Sussex Academic Press, pp. 98-117.

DAIUTE Colette (2014), Narrative Inquiry, Thousand Oaks (CA): Sage.

DARDY Claudine (1997), "L'identité-papier», Les Cahiers de médiologie, vol. 4, n 2, pp. 225-231.

DARLING Jonathan (2014), "Another letter from the Home Office: reading the material politics of asylum ", Environment and Planning: Society and Space, vol. 32, pp. 484-500.

DE Coulon Giada (2019). "Lillégalité régulière. Ethnographie du régime de l'aide d'urgence en Suisse, Lausanne: Antipodes.

Delgado Richard (1989), "Storytelling for Oppositionists and Others: A Plea for Narrative», Michigan Law Review, vol. 87, $\mathrm{n}^{\circ}$ 8, pp. 2411-2441.

DenIs Jérôme (2011), «Le travail de l'écrit en coulisses de la relation de service», @ctivités [en ligne], vol. 8, n 2, pp.32-52. En ligne: http:// activites.revues.org/2575 (consulté le 12 juillet 2017).

DEWEY John (1946), "Interaction and transaction», The Journal of Philosophy, vol. 43, $\mathrm{n}^{\circ}$ 19, pp. 505-517.

Di Donato Flora (2008a), La costruzione giudiziaria del fatto. Il ruolo della narrazione nel processo, Milan: Franco Angeli.

Di Donato Flora (2008b), "Client-lawyer Cooperation in the Construction of the Case», Cahiers de psychologie, Neuchâtel, $\mathrm{n}^{\circ} 44$, pp. 23-33. 
Di Donato Flora (2011), "Constructing Legal Narratives: ClientsLawyers' Stories», in Anne Wagner, Lee Cheng (éds), Exploring Courtroom Discourse, Surrey: Ashgate, pp. 111-131.

Di Donato Flora (2012a), La realtà delle storie. Tracce di una cultura, Naples: Guida.

Di Donato Flora (2012b), «Accessing Law Through the Humanities: Degrees of Agentivity When Actors are Natives or Immigrants. Comparing Southern Italy/North-west Switzerland", ISLL Papers, $\mathrm{n}^{\circ}$ 5. En ligne: http://www.lawandliterature.org/index. php?channel=CONTENTS\&year=2012.

Di Donato Flora (2014), "Narratives in Cultural Contexts. The Legal Agentivity of the Protagonists", SSRN Scholarly Paper, No. ID 2469436, Rochester (NY): Social Science Research Network. En ligne: http://dx.doi.org/10.2139/ssrn.2469436 (consulté le 5 septembre 2017).

Di DonAto Flora (2015), «Making in-Justices Visible. The Blindness of Bureaucracy", in Paolo Heritier (éd.), Teoria e critica della regolazione sociale. Special issue on Visiocracy, Milan: Mimesis, pp. 57-77.

Di Donato Flora (2016a), Lintegrazione degli stranieri in Svizzera. Genesi ed evoluzione dei significati giuridici, Milan : Mimesis.

Di Donato Flora (2016b), «Le récit comme outil d'analyse juridique: perspectives "top down" et "bottom up". L'intégration des étrangers en Suisse", in Flora Di Donato, Jean-Philippe Dunand et JeanJacques Aubert (éds), Dossier. Droit et Littérature: Actes du Séminaire thématique du CIHDDR. Université de Neuchâtel, 29 avril 2015, ISLL Papers. The Online Collection of the Italian Society for Law and Literature, vol. 8. En ligne: http://amsacta.unibo.it/5562/ (consulté le 17 novembre 2017).

Di Donato Flora (2018), «How to Increase the Role of Vulnerable People in Legal Discourse? Possible Answers from Law \& Humanities and Legal Clinics: Teaching Experiences from Italy \& from Switzerland", in Paolo Heritier et Flora Di Donato (éds), Teoria e critica della regolazione sociale. Special issue on Humanities and Legal Clinics. Law and Humanistic Methodology, Milan: Mimesis, pp. 35-55. Di Donato, Flora (2020), The Analysis of Legal Cases: A Narrative Approach, Londres/New York: Routledge.

Di Donato Flora, et MaHon Pascal (2009), «Federalism and "Cultural" Identities: Some Remarks on the Naturalization Procedure 
in Switzerland", in International Journal of Jurisprudence and Philosophy of Law, Ratio Iuris, vol. 22, n 2, pp. 281-294.

Di Donato Flora, et SCAMARdella Francesca (2013), «Epistemologia e processo. Un approccio di socio-clinical law", in Sociologia del diritto, vol. 3, pp. 75-109.

Di Donato Flora, et Scamardella Francesca (2016), Il metodo clinicolegale, Naples: ES.

Douglas Mary (2005), De la souillure. Essai sur les notions de pollution et de tabou, Paris: La Découverte (première édition 1996).

Duranti Alessandro (2007), Etnopragmatica. La forza nel parlare, Rome: Carocci.

DuBOIS Vincent (1999), La vie au guichet: relation administrative et traitement de la misère, Paris: Economica.

DuRKHeIM Emil (2002), Les règles de la méthode sociologique (Original 1894), Chicoutimi: J.-M. Tremblay. En ligne: http:// www.uqac.ca/zone30/Classiques_des_sciences_sociales/classiques/ Durkheim_emile/regles_methode/regles_methode.html.

DuveEN Gerard, et Lloyd Barbara (éds) (1990), Social representations and the development of knowledge, Cambridge: Cambridge University Press.

ECKERT Julia M. (éd.) (2008), The social life of anti-terrorism laws: the war on terror and the classifications of the "dangerous other", Bielefeld: transcript.

EHRlich Susan (2015), «Narrative, Institutional Processes, and Gendered Inequalities", in De Fina Anna et Alexandra Georgakopoulou (éds), The Handbook of Narrative Analysis, New York: John Wiley \& Sons, pp. 294-310.

EuIAs Norbert (1993), Engagement et distanciation. Contributions à la sociologie de la connaissance, Paris: Fayard.

Ellmann Stephen, Dinerstein Robert, Gunning Isabelle, Kruse Katherine, et SHALLECK Ann (2009), Lawyers and Clients. Critical Issues in Interviewing and Counseling, St Paul (MN): Thomson Reuters, American Casebook Series.

ERIKSON Erik H. (1959), Identity and the life cycle. Selected papers, New York: International Universities Press. En ligne: http://www.archive. org/details/identityandtheli011578mbp. 
ERIKSON Erik H. (1993), Adolescence et crise: la quête de l'identité, Paris: Flammarion.

Eteläpelto Anneli, VÄhäsantanen Katja, HöKKä Päivi, et PalOniemi Susanna (2013), "What is agency? Conceptualizing agency at work», Educational Research Review, vol. 10, pp. 45-65. Etudes de communication (2009), vol. 33, Lille: revue éditée par le centre de recherche GERiiCO.

Eule Tobias G. (2014), Inside immigration law: migration management and policy application in Germany, Ashgate: Routledge.

Ewick Patricia, et Silbey Susan (1992), "Conformity, Contestation and Resistance: An Account of Legal Consciousness", New England Law Review, vol. 26, n³, pp. 731-749.

Ewick Patricia, et Silbey Susan (1998), The Common Place of Law. Stories from Everyday Life, Chicago/Londres: The University of Chicago Press.

FACCHINETTI Thomas (2011), "Souveraineté politique et intégration interculturelle. Le cas de la République et Canton de Neuchâtel en Suisse", in Actes du Symposium international sur l'interculturalisme, Montréal. En ligne: www.symposium-interculturalisme.com (consulté le 17 novembre 2017).

FACCHINETTI Thomas (2012), «La notion d'intégration dans le droit suisse des migrations et dans les réformes en cours (LEtr, LAsi, LN)", in Cesla Amarelle (éd.), Lintégration des étrangers à l'épreuve du droit suisse. Fondements et applications pratiques, Berne: Stämpfli, pp. 61-80. FASSIN Didier (2008a), "L'éthique au-delà de la règle. Réflexions autour d'une enquête ethnographique sur les pratiques de soins en Afrique du Sud», Sociétés contemporaines 71, n 3, pp. 117-135.

FASsIN Didier (2008b), «La politique des anthropologues. Une histoire française", L'Homme 1-2, n 185-186, pp. 165-186.

FAVRET-SAADA Jeanne (1977), Les mots, la mort, les sorts: la sorcellerie dans le bocage, Paris: Galimard.

Ferraris Maurizio (2001), Documentalità. Perché è necessario lasciar tracce, Rome/Bari: Laterza.

FibBi Rosita (2012), "La pratique de l'intégration dans les cantons et la marge de manœuvre du fédéralisme», in Cesla Amarelle (éd.), L'intégration des étrangers à l'épreuve du droit suisse. Fondements et applications pratiques, Berne: Stämpfli, pp. 81-94. 
Fieulaine Nicolas, Kalampalikis Nikos, Haas Valérie, et Béal Arnaud (2013), «Les représentations sociales de l'appareil judiciaire: principes organisateurs, expériences et postures idéologiques ", Revue internationale de psychologie sociale, vol. 1, t. 26, pp. 35-59.

Fischer Nicolas (2007), «Entre urgence et contrôle», Recueil Alexandries, Collections Esquisses, février 2007. En ligne:[http://www. reseau-terra.eu/article 560.html].

FisCHER Nicolas, et DARLEY Mathilde (2010), «Le traitement de l'immigration, entre logique administrative et logique pénale», Champ pénal/Penal field, vol. VII. En ligne: http://champpenal.revues. org/7843; DOI : 10.4000/champpenal.7843 (consulté le 6 novembre 2017).

Flamant Nicolas (2005), «Observer, analyser, restituer. Conditions et contradictions de l'enquête ethnologique en entreprise», Terrain 44, pp. 137-152.

FLICK Uwe (1992), «Triangulation Revisited: Strategy of validation or alternative?", Journal for the Theory of Social Behaviour, vol. 22, $\mathrm{n}^{\circ} 2$, pp. 175-197.

Flyvbjerg Bent (2011), "Case study», in Norman K. Denzin et Yvonna S. Lincoln (éds), The Sage Handbook of Qualitative Research, $4^{\mathrm{e}}$ édition, Thousand Oaks (CA): Sage, pp. 301-316.

ForTiER Anne-Marie (2013), "What's the Big Deal? Naturalisation and the Politics of Desire", Citizenship Studies 17, n 6-7, pp. 697-711. Fossier Arnaud, et Monnet Éric (2009), «Que faire des institutions?», Tracés. Revue de Sciences humaines, vol. 17, n 2, pp. 7-28.

FRAENKEL Béatrice (2006), "Actes écrits, actes oraux: la performativité à l'épreuve de l'écriture", Études de communication, vol. 29, pp. 69-93.

Fraenkel Béatrice, Pontille David, Collard Damien, et Deharo Gaëlle (2010), Le travail des huissiers: transformations d'un métier de l'écrit, Toulouse: Octares.

Frauenfelder Arnaud (2007), Les paradoxes de la naturalisation. Enquête auprès de jeunes issus de l'immigration, Paris: L'Harmattan.

GADIENT Irma (2015), «Ausgrenzungen entgegnen. Ultranationalistes von Migrantinnen und Migranten in Ausweisungsprozessen im Kanton Genf um 1900 ", in Damir Skenderovic et Irma Gadient (éds), Revue suisse d'histoire, numéro thématique, "Histoire des migrations en Suisse; un changement de perspective», vol. 65, n 1, pp. 15-32. 
GARDEY Delphine (2008), Écrire, calculer, classer, Comment une révolution de papier a transformé les sociétés contemporaines (1800-1940), Paris: La Découverte.

Garufo Francesco (2015), L'emploi du temps, Lausanne: Antipodes. GEERTZ Clifford (2000 [1973]), The Interpretation of Cultures: Selected Essays, New York: Basic Books.

Giauque David, et ÉMERY Yves (2016), L'acteur et la bureaucratie au XXI ${ }^{e}$ siècle, Presses de l'Université Laval.

GILlespie Alex (2006a), Becoming other: From social interaction to self-reflection, Greenwich (CT): Information Age Publishing.

Gillespie Alex (2006b), «Games and the development of perspective taking", Human Development, vol. 49, n 2, pp. 87-92.

Gillespie Alex, et Cornish Flora (2010), «What can be said? Identity as a constraint on knowledge production ", Papers on Social Representations, vol. 19, par. 5.1-5.13.

Glick Schiller Nina, et Caglar Ayse (2013), «Locating Migrant Pathways of Economic Emplacement: Thinking Beyond the Ethnic Lens", Ethnicities 13, n 4, pp. 494-514.

Gillespie Alex, et ZitToun Tania (2010), «Using Resources: Conceptualizing the Mediation and Reflective Use of Tools and Signs», Culture \& Psychology, vol. 16, n 1, pp. 37-62.

Goffman Erwin (1961), Asylums: Essays on the Social Situation of Mental Patients and Other Inmates, New York: Anchor Books.

GofFMAn Erwin (1991), Les cadres de l'expérience, Paris: Les Éditions de Minuit.

GofFMan Erwin (1997), "Status, territory, and the self. From the "Territories of the self" ", in Charles Lemert et Ann. Branaman (éds), The Goffman reader (première édition 1971). Malden (MA)/Oxford: Blackwell Publishing, pp. 45-54.

Graeber David (2012), «Dead zones of the imagination. On violence, bureaucracy, and interpretive labor", $H A U$ : Journal of Ethnographic Theory, vol. 2, n² 2, pp. 105-128.

Graeber David (2015), The Utopia of Rule. On Technology, Stupidity, and the Secret Joys of Bureaucracy, New York/Londres: Melville Press. 
GRISEL Étienne (1989), "ad art. 44 », in Commentaire de la Constitution fédérale de la Confédération suisse, Bâle/Berne/Zurich: Helbing \& Lichtenhahn/Schulthess/Staempfli \& Cie, 1986-1995.

Grosjean Michèle, et Lacoste Michèle (1998), "L'oral et l'écrit dans les communications de travail ou les illusions du "tout écrit", Sociologie du travail, vol. 40, n 4, pp. 439-465.

Gusfield Joseph R. (1984), The Culture of Public Problems: DrinkingDriving and the Symbolic Order, Chicago: University of Chicago Press. GUTWIRTH Serge (2013), «Le contexte du droit ce sont ses sources formelles et les faits et moyens qui exigent son intervention", Revue interdisciplinaire d'études juridiques - Droit en contexte, vol. 70, n. 1, pp. 108-116.

GutzWILler Céline (2008), Droit de la nationalité et fédéralisme en Suisse, Genève: Schulthess.

GuTZWILLER Céline (2012), L'intégration dans la loi sur la nationalité: étude de cas en matière de naturalisation ordinaire, in Cesla Amarelle (éd.), L'intégration des étrangers à l'épreuve du droit suisse. Fondements et applications pratiques, Berne: Stämpfli Éditions, pp. 131-147.

GutzWILler Céline (2014), "ad art. 15b LN», in Cesla Amarelle et Minh Son Nguyen (éds), Code annoté de droit des migrations. Vol. V: Loi sur la nationalité (LN), Berne: Stämpfli Éditions SA, pp. 78-80.

GutzWILler Céline (2015), "La loi fédérale sur la nationalité du 20 juin 2014: les conditions de naturalisation", in Martine Dang, Rahel Diethelm et Minh Son Nguyen (éds), Actualité du droit des étrangers, vol. 1, Bâle-Neuchâtel: Helbing \& Lichtenhahn, pp. 1-26.

HaAvisto Vaula (2001), "Breaking the Courtroom Code: Client Initiatives in Finnish Civil Hearings", International Journal for the Semiotics of Law, vol. 15, n 4, pp. 399-409.

Henare Amiria, HolbraAd Martin, et Wastell Sari (2006), Thinking through things: theorising artefacts ethnographically, Londres: Routledge. Henning, Christoph (2007), "Institution", in George Ritzer (éd.), The Blackwell Encyclopedia of Sociology, Oxford (UK), Malden (USA) Carlton (Australia): Blackwell Publishing Ltd. En ligne: [http://doi. wiley.com/10.1111/b.9781405124331.2007.x]).

Hertz Ellen, Martin Hélène, et Valli Marcelo (2004), Le «feeling» des agents de l'État providence. Analyse des logiques sous-jacentes 
aux régimes de l'assurance chômage et de l'aide sociale, Ethnologie française 2002/2 - Tome XXXVII, pp. 221-231.

Houdart Sophie, et Thiéry Olivier (2011), Humains, non-humains. Comment repeupler les sciences sociales?, Paris: La Découverte.

Hull Mathew S. (2012), Government of Paper: The Materiality of Bureaucracy in Urban Pakistan, Berkeley: University of California Press.

JACOBSSON Katarina (2016), "Analysing documents through fieldwork", in David Silverman (éd.), Qualitative Research, Londres: Sage, pp. 155-170.

JAMES William (1904), "Qu'entend-on par pragmatisme?», in (2007) Le pragmatisme (Nathalie Ferron, trad.), Paris: Flammarion, pp. 111-140.

JAMES William (2007), The principles of psychology (Original 1890 Vol. II), New York: Cosimo Classic.

Jeannerat Éloi, et Mahon Pascal (2014), «ad art. 112 LEtr», in Cesla Amarelle et Minh Son Nguyen (éds), Code annoté de droit des migrations. Vol. II: Loi sur les étrangers (LEtr), Berne: Stämpfli Éditions SA, pp. 1285-1297.

JoDelet Denise (1989/2003), Les représentations sociales, Paris: PUF.

Jodelet Denise (2006), «Place de l'expérience vécue dans le processus de formation des représentations sociales", in Valérie Haas (éd.), Les savoirs du quotidien. Transmission, appropriations, représentations, Rennes: Presses universitaires de Rennes, pp. 235-255.

JoDELET Denise (2008), «Le mouvement de retour vers le sujet et l'approche des représentations sociales", Connexions, vol. 89, no. 1, pp. 25-46.

Jodelet Denise (2015), Représentations sociales et mondes de vie, Paris: Éditions des Archives contemporaines.

Kalampalikis Nikos, et Apostolidis Themis (2016), "La perspective sociogénétique des représentations sociales", in Grégory Le Monaco, Sylvain Delouvée et Patrick Rateau (éds), Les représentations sociales, Bruxelles: De Boeck, pp. 69-84.

Kapardis Andreas (2014), Psychology and law. A critical introduction, 4 édition, Cambridge: Cambridge Universiy Press.

Kaufmann Jean-Claude (1996), L'entretien compréhensif, Paris: Nathan. 
Kelly Tobias (2008), "Documents, Security and Suspicion: The Social Production of Ignorance in the Social Life of Anti-Terrorism Laws», in Julia Eckert (éd.), The War on Terror and the Classifications of the "Dangerous Other", Bielefeld: transcript Verlag, pp. 109-131.

Krieg-Planque Alice, et Ogier Claire (2010), «Discours institutionnels. Perspectives pour les sciences de la communication». Mots. Les langages du politique, $\mathrm{n}^{\circ}$ 94, pp.91-96.

Kubal Agnieszka (2013), "Migrants» Relationship with Law in the Host Country: Exploring the Role of Legal Culture», Journal of Intercultural Studies, vol. 34, n 1, pp. 55-72.

LATOUR Bruno (1994), «Une sociologie sans objet? Remarques sur l'interobjectivité», Sociologie du travail, vol. 36, pp. 587-607.

Latour Bruno (1995), "The Pedofil of Boa Vista. A Photophilosophical Montage», Common Knowledge, 3, pp. 147-187.

Latour Bruno (1996), "On Interobjectivity», Mind, Culture, and Activity, vol. 3, n 4, pp. 228-245.

LATOUR Bruno (2002), La Fabrique du droit. Une ethnographie du Conseil d'État, Paris: La Découverte.

LATOUR Bruno (2005), Reassembling the social: an introduction to actornetwork-theory, Oxford and New York: Oxford University Press.

LATOUR Bruno (2006), Changer la société, refaire la sociologie, Paris: La Découverte.

Latour Bruno, et Woolgar Steeve (1988), La vie de laboratoire, Paris: La Découverte.

LAURENS Sylvain (2008), «Les agents de l'État face à leur propre pouvoir. Éléments pour une microanalyse des mots griffonnés en marge des décisions officielles", Genèses, vol. 3, n 72, pp. 26-41.

LaVAnchy Anne (2012a), "Les mariages forcés dans le Canton de Vaud», Lausanne: CCLVD, 2011.

LAVANCHY Anne (2012b), "Par amour du même. La production de l'homogamie par les employés d'état civil en Suisse», Présentation à l'atelier L'amour et ses frontières: la régulation étatique des mariages transnationaux dans l'espace Schengen, Université libre de Bruxelles, Belgique.

LAVANCHY Anne (2013a), "Dissonant alignments. The ethics and politics of researching state institutions", Current Sociology, vol. 61, $n^{\circ}$ 5-6, pp. 677-692. 
LAVANCHY Anne (2013b), «L'amour aux services de l'état civil: régulations institutionnelles de l'intimité et fabrique de la ressemblance nationale en Suisse", Migration sociétés, vol. 25, n 150, pp. 61-94.

LAVANCHY Anne (2014a), «Die Gefühlswelt des Gesetzes: die kritische Umsetzung von eherechtlichen Vorschriften im Zivilstandsamt", Fampra - Die Praxis des Familienrechts, vol. 15, n 1, pp. 92-117.

LAVANCHY Anne (2014b), "How does "Race" matter in Switzerland?", Working Papers MAPS. The circulation of people (2014-7/E). En ligne: [http://www2.unine.ch/maps/workingpapersmaps\#cid317406].

LAVANCHY Anne (2015), "Glimpses into the Hearts of Whiteness: How Intimacy Institutions Give Shape to the Desirable Nationals in Switzerland», in Patricia Purtschert et Harald Fischer-Tiné (éds), Colonial Switzerland. Rethinking Colonialism from the Margins, London et al.: Palgrave Macmillan, pp. 278-296.

LAVANCHY Anne (2020), "Quand l'administration fait le couple par le corps. Les effets du mariage civil en Suisse», Émulation, 32, pp. 63-73. Le Fort Olivia, et CARron Djemila (2016), «L'enseignement clinique du droit à Genève», Jusletter, 20 juin 2016.

LEONTIEV Dimitry A. (1996), "Dimensions of the meaning/sense concept in the psychological context», in Charles W. Tolman, Frances Cherry, René van Hezewijk et Ian Lubek (éds), Problems of theoretical psychology, North York, Ontario: Captus University Publications, pp. 130-144.

LESERVOISIER Olivier (éd.) (2005), «L'anthropologie réflexive comme exigence épistémologique et méthodologique», in Terrains ethnographiques et hiérarchies sociales. Retour réflexif sur la situation d'enquête, Paris: Karthala.

LEVASSEUR Élodie, et HaAs Valérie (2015), "Quand un interviewé.e raconte son souvenir de l'inondation. Une mise en récit qui interroge la légitimité à raconter et à questionner", in Sarah Cordonnier (éd.), Trajectoire et témoignage. Pour une réflexion pluridisciplinaire, Paris: Éditions des Archives contemporaines, pp. 43-56.

LIPSKY Michael (1980), Street-level bureaucracy. Dilemmas of the Individual in Public Services, New-York: Russell Sage Foundation.

Lofland John, Snow David A., Anderson Leon, et Lofland Lyn H. (éds) (2006), Analyzing Social Settings: A Guide to Qualitative Observation and Analysis, $4^{\mathrm{e}}$ édition, Belmont, CA: Wadsworth. 
López Gerald P. (1992), Rebellious Lawyering: One Chicano's Vision of Progressive Law Practice, San Francisco: Westview Press.

López Gerald P. (2017), «Transform-Don't Just Tinker With-Legal Education», 23 Clinical L. Rev., 471, 576.

LOWENKRON Laura, et FERREIRA Leticia (2014), «Anthropological perspectives on documents: Ethnographic dialogues on the trail of police papers", Vibrant - Virtual Brazilian Anthropology, vol. 11, $\mathrm{n}^{\circ} 2$, pp. 75-111.

Luban David (2007), Legal Ethics and Human Dignity, Cambridge: Cambridge University Press.

Mahon Pascal (1984), Réglementation du travail, Lausanne: Presses polytechniques fédérales.

Mahon Pascal (2003), "ad art. 38», in Jean-François Aubert et Pascal Mahon (éds), Petit commentaire de la Constitution fédérale de la Confédération suisse du 18 avril 1999, Zurich-Bâle-Genève: Schulthess, pp. 344-357.

Mahon Pascal (2003), "ad art. 121», in Jean-François Aubert et Pascal Mahon (éds), Petit commentaire de la Constitution fédérale de la Confédération suisse du 18 avril 1999, Zurich-Bâle-Genève: Schulthess, pp. 960-974.

Mahon Pascal, et Collette Marlène (2011), «La notion d'intégration des étrangers en droit suisse", in Pascal Mahon et Minh Son Nguyen (éds), L'activité et l'espace, Droit du sport et aménagement du territoire. Mélanges en l'honneur de Piermarco Zen-Ruffinen, Bâle-Neuchâtel: Helbing \& Lichtenhahn, pp. 225-243.

Malinverni Giorgio (1987), "ad art. 70», in Commentaire de la Constitution fédérale de la Confédération suisse, Bâle-Berne-Zurich: Helbing \& Lichtenhahn, Schulthess, Staempfli \& Cie, 1986-1995.

Malinverni Giorgio (1995), "ad art. 69ter», in Commentaire de la Constitution fédérale de la Confédération suisse, Bâle-Berne-Zurich: Helbing \& Lichtenhahn, Schulthess, Staempfli \& Cie, 1986-1995.

Marková Ivana (2007), Dialogicité et représentations sociales, Paris: Presses universitaires de France.

Martin Hélène, Hertz Ellen, et Valdi Marcelo (2004), «Le "feeling” : une logique sous-jacente au fonctionnement de l'État providence", FEAS, Aspects de la sécurité sociale, vol. 1, pp. 12-21. 
MARTin Jack, et Gillespie Alex (2010), «A neo-meadian approach to human agency: Relating the social and the psychological in the ontogenesis of perspective-coordinating persons", Integrative Psychological and Behavioral Science, vol. 44, n 3, pp. 252-272.

Maskens Maïté (2015), «Bordering Intimacy. The Fight against Marriages of Convenience in Brussels", The Cambridge Journal of Anthropology, vol. 33, $\mathrm{n}^{\circ}$ 2, pp. 42-58.

MASSARO Tony (1989), «Empathy Legal Storytelling, and the Rule of Law: New Words, Old Wounds?", The Michigan law Review, vol. 87, $\mathrm{n}^{\circ} 8$, pp. 2099-2127.

May Peter, et Winter Søren (2009), "Politicians, Managers, and Street-Level Bureaucrats: Influences on Policy Implementation", Journal of Public Administration Research and Theory, vol. 19, $\mathrm{n}^{\circ} 3$, pp. 453-476.

Mayère Anne, BAZET Isabelle, et Roux Angélique (2012), "Zéro papier" et "pense-bêtes" à l'aune de l'informatisation du dossier de soins", Revue d'anthropologie des connaissances, vol. 6, $\mathrm{n}^{\circ} 1$, pp. 115-139.

Mead George Hubert (1934), Mind, Self and Society, from the Standpoint of a Social Behaviorist (C. W. Morris, éd.), Chicago: University of Chicago Press.

Mehan Hugh, Hertweck Almq, et Meinls J. Lee (1986), Handicapping the Handicapped: Decision Making in Students' Educational Careers, Stanford: Stanford University Press.

Menmeti Teuta (2013), «Réussite scolaire de jeunes femmes kosovares: quels processus psychosociaux?", Dossiers de psychologie et éducation, vol. 70, pp. 5-125.

Merry Sally E. (2003), "Rights Talk and the Experiences of Law: Implementing Women's Human Rights to Protection from Violence», Human Rights Quarterly, n² 25, pp. 343-381.

Merton Robert K. (1949), Social Theory and Social Structure, Glencoe: Free Press.

Merzeau Louise (2011), Pour une médiologie de la mémoire. Habilitation à diriger des recherches. Sciences de l'information et de la communication. Université de Nanterre - Paris X.

MongKol Jaroenjit (2014), «Description of legal consciousness of the supporting officers' duty performances in Nakhon Reatchasima 
Rajabhat University», Review of Integrative Business \& Economics, vol. 3, pp. 28-33.

Moret Joëlle (2006), Somali Refugees in Switzerland: Strategies of Exile and Policy Responses, Neuchâtel: SFM.

Moret Joëlle (2017), «Mobility Capital: Somali Migrants' (Im) Mobilities Trajectories and the Negotiation of Social Inequalities across Borders", in Geoforum.

Moscovici Serge (1984), Psychologie sociale, Paris: PUF.

NADER Laura (1965), The Ethnography of Law, Menasha, Wisconsin: American Anthropological Association.

Nader Laura (1997), "Controlling Processes. Tracing the Dynamic Components of Power", Current Anthropology 38, n 5, pp. 711-736.

NADER Laura (2002), The life of law. Anthropological projects, Berkeley: University of California Press.

NGuyen Minh Son (2017), "ad art. 1 LEtr», in Cesla Amarelle et Minh Son Nguyen (éds), Code annoté de droit des migrations, Vol. II: Loi sur les étrangers (LEtr), Berne: Stämpfli Éditions, pp. 1-5.

Niederberger Joseph M. (2005), «Le développement d'une politique intégration suisse», in Hans Mahnig (éd.), Histoire de la politique de migration, d'asile et d'intégration en Suisse depuis 1948, Zurich: Seismo, pp. 255-288.

Norman Donald (1993), "Les artefacts cognitifs», Raisons pratiques, $\mathrm{n}^{\circ} 4$, pp. 15-34.

O’Barr William M., et Conley John M. (1988), «Lay Expectations of the Civil Justice System", Law and Society Review, vol. 22, $\mathrm{n}^{\circ} 1$, pp. 137-162.

OgIEN Albert, et QuÉRÉ Louis (2005), Le vocabulaire de la sociologie de l'action, Paris: Ellipses.

Ogloff James R. P., Beavers Donna J., et DeLeon Patrick H. (1999), "Psychology and the law: A shared vision for the 21st century", Professional Psychology: Research and Practice, vol. 30, $\mathrm{n}^{\circ} 4$, pp. 331-332.

Olivier de SARDAN Jean-Pierre (2008), La rigueur du qualitatif: les contraintes empiriques de linterprétation socio-anthropologique, Louvain-la-Neuve: Academia Bruylant. 
Olivier de Sardan Jean-Pierre (2000), "Le "Je" méthodologique: implication et explicitation dans l'enquête de terrain ", Revue française de sociologie 41, $\mathrm{n}^{\circ}$ 3, pp. 417-445.

Olson Greta (2015), «Futures of Law and Literature. A Preliminary Overview from a Culturalist Perspective», in Christina Hiebaum, Susanne Knaller et Doris Pichler (éds), Recht und Literatur im Zwischenraum/Law and Literature In-Between: Aktuelle inter-und transdisziplinäre Zugänge/Contemporary Inter- and Transdisciplinary Approaches, Bielefeld: transcript, pp. 37-69.

O’ReILly Karen (2005), Ethnographic Methods, London and New York: Routledge.

Ossipow Laurence (1996), "Citoyenneté et nationalité: pratiques et représentations de l'intégration en Suisse chez des candidats à la naturalisation et des responsables de la procédure», in Hans-Rudolf Wicker, Jean-Luc Alber, Claudio Bolzman, Rosita Fibbi, Kurt Imhof et Andreas Wimmer (éds), Das Fremde in der Gesellschaft: Migration, Ethnizität und Staat/L'altérité dans la société: migration, ethnicité, État, Zurich: Seismo, pp. 229-242.

Paillé Pierre, et Mucchieldi Alex (2016), L'analyse qualitative en sciences humaines et sociales, Paris: Armand Colin.

PéLISSE Jérôme (2005), «A-t-on conscience du droit? Autour des Legal Consciousness Studies", Genèses, vol. n 59, n² 2, pp. 114-130.

Perret-Clermont Anne-Nelly, et Zittoun Tania (2002), «Esquisse d'une psychologie de la transition", Éducation Permanente, $\mathrm{n}^{\circ} 1$, pp. 12-14.

Phenninx Rinus (2003, octobre), "Integration: The Role of Communities, Institutions, and the State». En ligne: [http://www. migrationpolicy.org/article/integration-role-communities-institutions-and-state], consulté le 24 mars 2016.

PIGUET Étienne (2009), L'immigrazione in Svizzera. Sessant'anni con la porta semiaperta, Bellinzona: Casagrande.

PribytKova Elena (2016), "A Decent Social Minimum as a Matter of Justice», in Helmut P. Gaisbauer, Gottfried Schweiger et Clemens Sedmak (éds), Ethical Issues in Poverty Alleviation, Springer International Publishing AG Switzerland, pp. 43-56.

PrIOR Lindsay (2016), "Documents in Social Research", in David Silverman (éd.), Qualitative Research, Londres: Sage, pp. 155-170. 
Rochex Jean-Yves (1998), Le sens de l'expérience scolaire, Paris: Presses universitaires de France.

Roelun Laura (2012), "Je demande la naturalisation parce que...". Analyse argumentative des motivations qui ont décidé les candidats à poser une demande de naturalisation (mémoire de master), Neuchâtel: Université de Neuchâtel. En ligne: [https://doc.rero.ch/record/30845/ files/memoire_roellin.pdf].

Saunders Benjamin, Kitzinger Jenny, et Kitzinger Celia (2015), "Anonymising Interview Data: Challenges and Compromise in Practice», Qualitative Research 15, n 5, pp. 616-632.

SAUSER-Hall Georges (1914), La nationalisation des étrangers en Suisse, Neuchâtel: Attinger Frères.

Sauser-Hall Georges (1921), La nationalité en droit suisse, Berne: K. J. Wyss Erben.

SCAmardella Francesca, et Di Donato Flora (2012), "Il silenzio "a più voci" nelle narrazioni di clienti e avvocati", Sociologia del diritto, (2), pp. 127-150.

SCHNAPPER Dominique (2007), Qu'est-ce que lintégration, Paris: Gallimard.

SChreIER Margit (2013), "Qualitative Content Analysis», in Uwe Flick (éd.), The Sage handbook of qualitative data analysis, London: Sage Publications Ltd, pp.170-183.

ScotT James (1998), Seeing like a State: How certain schemes to improve the human condition have failed, Yale University Press.

ScotT Susan (2011), Total Institutions and Reinvented Identities, New York: Palgrave Macmillan.

SHAW Jan, et KaYE Mike (2013), A Question ofCredibility: Why So Many Initial Asylum Decisions are Overturned on Appeal in the UK, London: Amnesty International. http://www.refworld.org/docid/518120c64. html (consulté le 15 février 2016).

Shaw Julia, Öhman Lisa, et van Koppen Peter (2013), «Psychology and law: The past, present, and future of the discipline", Psychology, Crime \& Law, vol. 19, n 8, pp. 643-647.

SHERWIN Richard (1993), "The Narrative Construction of Legal Reality», Vermont Law Review, vol. 18, pp. 88-120.

SHERWIN Richard (2012-2013), Visual Jurisprudence, 57 N.Y.L. Sch. L. Rev. 11. 
Silbey Susan (2008), "Legal Consciousness», in Peter Cane et Joanne Conaghan (éds), The New Oxford Companion to Law, Oxford: Oxford University Press.

Silbey Susan (2014), "The Courts in American Public Culture», Daedalus, The Journal of the American Academy of Arts \& Sciences, pp. 140-156.

SOMERS Margareth R. (1994), "The Narrative Constitution of Identity: A Relational and Network Approach", Theory and Society, vol. 23, n 5, pp. 605-649.

Sow Dieyla, et Mahon Pascal (2014), «ad art. 14 LN», in Cesla Amarelle et Minh Son Nguyen (éds), Code annoté de droit des migrations, Volume V: Loi sur la nationalité (LN), Berne: Stämpfli Éditions, pp. 45-60.

SpIre Alexis (2007), «L'asile au guichet. La dépolitisation du droit des étrangers par le travail bureaucratique", Actes de la recherche en sciences sociales, vol. 4, $\mathrm{n}^{\circ} 169$, pp. 4-21.

SPIRE Alexis (2008), Accueillir ou reconduire. Enquête sur les guichets de l'immigration, Paris: Raisons d'agir.

SPIRE Alexis, et FisCHER Nicolas (2009), "L'État face aux illégalismes», Politix, vol. 87, n 3, pp. 7-20.

SPIVAK Gayatri Chakravorty (1988), "Can the Subaltern Speak?», in C. Nelson et L. Grossberg (éds), Marxism and the Interpretation of Culture, pp. 271-313.

STUDER Brigitte (2004), "Die Ehefrau, die den Ausländer heiratet, soll sich die Geschichte klar überlegen": Geschlecht, Ehe und nationale Zugehörigkeit im 20. Jahrhundert in der Schweiz», Tsantsa, $\mathrm{n}^{\circ}$ 9, pp. 49-60.

STUDER Brigitte (2013), «D’une politique de naturalisation restrictive à une politique intégratrice? 1934-2004", in Brigitte Studer, Gérard Arlettaz et Regula Argast (éds), Le droit d'être suisse. Acquisition, perte et retrait de la nationalité de 1848 à nos jours, Lausanne: Antipodes, pp. 117-177.

STUDER Brigitte, ArLeTtaz Gérard, et ARGaST Regula (éds) (2013), Le droit d'être suisse. Acquisition, perte et retrait de la nationalité de 1848 à nos jours, Lausanne: Antipodes.

Telfer Jonathan (2004), «Dissent and Consent: Negotiating the Adoption Triangle», in Lynne Hume et Jane Mulcock (éds), 
Anthropologists in the Field: Cases in Participant Observation, New York: Columbia University Press, pp. 71-81.

ToOmela Aaro (2007), "Sometimes one is more than two: when collaboration inhibits knowledge construction", Integrative Psychological o Behavioral Science, vol. 41, n 2, pp. 198-207.

Torpey John (1997), "Coming and Going: On the State Monopolization of the Legitimate "Means of Movement" ", Sociological Theory, pp. 239-259.

Turner Victor (1969), Ritual Process. Structure and Anti-Structure, New York: Aldine De Gruyter.

VAlsiner Jaan (2000), Culture and Human Development, Thousand Oaks, CA: Sage.

VALSINER Jaan (2014), An invitation to cultural psychology, London: Sage.

VALSINER Jaan, et Rosa Alberto (éds) (2007), The Cambridge handbook of sociocultural psychology, Cambridge University Press.

VINCK Dominique (2009), «De l'objet intermédiaire à l'objetfrontière. Vers la prise en compte du travail d'équipement", Revue d'anthropologie des connaissances, vol. 3, 1, pp. 51-72.

Volpp Leti (2006), "Quand on rend la culture responsable de la mauvaise conduite», Nouvelles Questions Féministes, vol. 25, $\mathrm{n}^{\circ} 3$, pp. 14-31.

WELLER Jean-Marc (2009), "Les agents administratifs: travail d'arbitrage et conscience professionnelle», in Didier Demazière (éd.), Sociologie des groupes professionnels, Paris: La Découverte, pp. 321-331. Weller Jean-Marc (2013), «Enquêter sur l'administration ou l'énigme du travail administratif", in Jean-Michel Eymeri-Douzans et Geert Bouckaert (éds), La France et ses administrations. Un état des savoirs, Bruxelles: Bruylant, pp. 211-236.

White Hayden (1981), "The Value of Narrativity in the Representation of Reality", Critical Inquiry, vol. 7, n 1, pp. 5-27. White James Boyd (1990), Justice as Translation, Chicago: The University of Chicago Press.

White Lucie E. (1997), "The Transformative Potential of Clinical Legal Education", Osgoode Hall Law Journal, 35 (3-4), pp. 603-611. 
Wichmann Nicole, Hermann Michael, D’Amato Gianni, EfionaylMäder Denise, Fibbi Rosita, Menet Joanna, et Ruedi Didier (2011), «Les marges de manœuvre au sein du fédéralisme: la politique de migration dans les cantons ", Neuchâtel: Swiss Forum for Migration.

Wichman Nicole (2013), «Existe-t-il une approche romande à l'intégration des étrangers?», in Forum Migration et intégration: focus sur la Suisse Romande, $\mathrm{n}^{\circ}$ 8, Neuchâtel.

WILSON Richard (2017), The Global Evolution of Clinical Legal Education More than a Method, Cambridge (MA): Cambridge University Press.

Wimmer Andreas (2011). "A Swiss Anomaly? A Relational Account of National Boundary-Making. Nations and Nationalism ", vol. 17, $\mathrm{n}^{\circ} 4$, pp. 718-737.

Windisch Uli (éd.) (2002), Suisse-Immigrés. Quarante ans de débats 1960-2001, Lausanne: L'Âge d'Homme.

WinnicotT Donald Woods (2002), Jeu et réalité. L'espace potentiel (C. Monod et J.-B. Pontalis, trad.), Paris: Gallimard.

Womersley Gail, Kloetzer Laure, et Gogikian Ratcliff Betty (2017), «Mental Health Problems Associated with Asylum Procedures of Refugees in European Countries", Nccr - on the Move, Highlights, vol. 2 (October 2017), pp. 37-40 (consulté à l'adresse [https://doi. org/10.4414/smw.2010.13110]).

Zimmer Oliver (2011), "Coping with Deviance: Swiss Nationhood in the Long Nineteenth Century», Nations and Nationalism 17, no. 4, Switzerland. A Nation-State or a Multinational State?, pp. 756-774. Zittoun, Tania (2006), Insertions. À quinze ans, entre échecs et apprentissage, Berne: Peter Lang.

Zittoun Tania, Baucal Aleksandar, Cornish Flora, et Gillespie Alex (2007), "Collaborative research, knowledge and emergence", Integrative Journal for Psychological and Behavioral Science, vol. 41, $\mathrm{n}^{\circ} 2$, pp. 208-217.

Zittoun Tania, et Perret-Clermont Anne-Nelly (2009), "Four social psychological lenses for developmental psychology", European Journal for Psychology of Education, vol. 24, n 2, pp. 387-403.

ZiTTOuN Tania (2012), "Lifecourse: A socio-cultural perspective», in Jaan Valsiner (éd.), The Oxford Handbook of Culture and Psychology, Oxford: Oxford University Press. 
Zittoun Tania, Valsiner Jaan, Vedeler Dankert, Salgado João, Gonçalves Miguel, et FerRING Dieter (2013), Human development in the lifecourse. Melodies of living, Cambridge: Cambridge University Press.

ZitToun Tania, et Gillespie Alex (2015a), «Integrating experiences: Body and mind moving between contexts", in Brady Wagoner, Nandita Chaudhary et Pernille Hviid (éds), Integrating experiences: Body and mind moving between contexts, Charlotte, NC: Information Age Publishing, pp. 3-49.

ZitToun Tania, et Gillespie Alex (2015b), "Transitions in the lifecourse: Learning from Alfred Schütz», in Amrei C. Joerchel et Gerhard Benetka (éds), Biographical ruptures and their repairs: Cultural transitions in development, Charlotte, NC: Information Age Publishing, pp. 147-157.

ZitToun Tania, et DE Saint-Laurent Constance (2015), "Lifecreativity: Imagining one’s life», in Vlad P. Glăveanu, Alex Gillespie et Jaan Valsiner (éds), Rethinking creativity: Contributions from cultural psychology, Hove/New York: Routledge, pp. 58-75.

ZitToun Tania, et Gillespie Alex (2016), Imagination in human and cultural development, London: Routledge.

ZitToun Tania (2017), «Modalities of generalization through single case studies", Integrative Psychological and Behavioral Science, vol. 51, n 2, pp. 171-194. 



\section{TABLE DES MATIÈRES}

AVANT-PROPOS

PARTIE I. INTRODUCTION

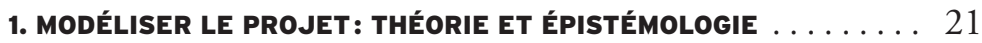

1.1 De l'intégration.................... 21

1.2 Approcher une réalité complexe ............ 23

1.2 .1 L'analyse juridique .................... 24

1.2.2 L'approche socioclinique du droit............ 26

1.2.3 L'anthropologie des institutions ............ 28

1.2.4 La psychologie socioculturelle ............. 32

1.2.5 Complémentarité .................... 33

1.3 La problématique $\ldots \ldots \ldots \ldots \ldots \ldots \ldots \ldots \ldots \ldots$

1.4 Un modèle: analyse des trajectoires d'intégration..... 35

1.4.1 La trajectoire administrativo-légale........... 36

1.4.2 La trajectoire de vie. . . . . . . . . . . . . . 39

1.4.3 Les transactions....................... 41

1.5 Synthèse et plan de l'ouvrage. ............. 43

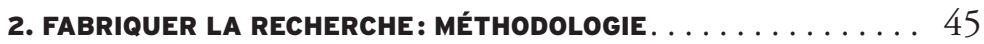

2.1 Une méthodologie qualitative interdisciplinaire ..... 45

2.1.1 Le travail collaboratif ................... 47

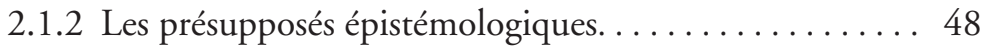

2.1 .3 Une démarche qualitative $\ldots \ldots \ldots \ldots \ldots \ldots \ldots \ldots$

2.2 Le travail de terrain et de production des données .... . 51

2.2.1 Un terrain ancré dans le canton de Neuchâtel....... . 51

2.2.2 Les données........................ 52

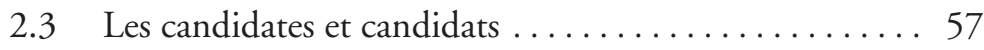




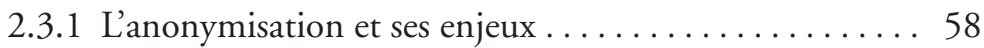

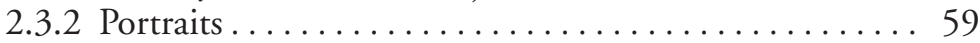

2.4 Les membres des administrations . . . . . . . . . . . 65

2.5 Des données empiriques aux cas ............. 68

2.6 Enjeux éthiques: les impacts de la recherche........ 69

2.6.1 Les données et leur protection. . . . . . . . . . . . . 69

2.6.2 Les attentes ...................... 70

2.6.3 Nos positionnements . . . . . . . . . . . . . . . 71

2.7 Synthèse: un terrain construit en collaboration ...... 73

PARTIE II. CONSTRUCTION DU CONTEXTE INSTITUTIONNEL . . . . . . 75

3. RETRACER LA NOTION D'INTÉGRATION: HISTOIRE DU DROIT . . . 77

3.1 Observations introductives et méthodologiques ...... 77

3.2 Genèse et évolution de la notion d'intégration dans l'ordre juridique suisse . . . . . . . . . . . . 80

3.2.1 1848-1890: la Suisse, pays d'émigration ........... 81

3.2.2 1890-1920: l'incorporation par le biais du «ius soli». . 84

3.2.3 1920-1950: l'introduction des permis de séjour et d'établissement ....................... 90

3.2.4 1950-1975: l'aptitude de la personne étrangère à devenir citoyenne. . . . . . . . . . . . . . . . 91

3.2.5 1976-1990: l'intégration des travailleuses et des travailleurs étrangers . . . . . . . . . . . 95

3.2.6 1990-2015: l'intégration comme tâche de l'État et devoir de la personne étrangère, ou encourager et exiger . . . . . 98

3.3 Synthèse: de l'incorporation à l'intégration comme institution de réinvention. . . . . . . . . . . 103

\section{DÉFINIR L'INTÉGRATION:}

SIGNIFICATIONS LÉGALES ACTUELLES . . . . . . . . . . . . . . . . 107

4.1 L'ubiquité de la notion d'intégration ............ 108

4.2 La notion d'intégration dans la loi fédérale sur la nationalité (LN) . . . . . . . . . . . . . . . . . . . . . . . . 109

4.2.1 1952-1990: la révision de l'article $14 \mathrm{LN}$ et l'introduction du critère de l'intégration ......... 109

4.2.2 Le concept d'intégration réussie dans la loi sur la nationalité du 20 juin $2014 \ldots \ldots \ldots \ldots \ldots \ldots$

4.2.3 Les critères de la naturalisation dans la pratique et la jurisprudence fédérales. . . . . . . . . . . . 116 
4.2.4 La protection juridictionnelle

en cas de refus de la naturalisation . . . . . . . . . . . 122

4.2 .5 Synthèse intermédiaire . . . . . . . . . . . . . . 125

4.3 La notion d'intégration dans la loi fédérale sur les étrangers (LEtr) . . . . . . . . . . . . . . . . . 126

4.3.1 Les conditions de l'intégration . . . . . . . . . . 127

4.3.2 L'intégration ne coïncide pas avec l'assimilation: le rôle de la jurisprudence . . . . . . . . . . . . . . 133

4.3.3 La protection juridictionnelle des personnes étrangères . . 135

4.3.4 Synthèse intermédiaire. . . . . . . . . . . . . . . . 137

4.4 La loi sur l'intégration et la cohésion culturelle du canton de Neuchâtel. . . . . . . . . . . . . . . . . . . 138

4.4 .1 Le principe d'égale dignité.................. 140

4.4.2 Les instruments d'une politique libérale: la Charte de la citoyenneté. . . . . . . . . . . . . . 141

4.4.3 La mise en œuvre de la loi : le COSM ............. 143

4.5 Synthèse : le défi de l'intégration réussie.......... . 144

5. DÉMÊLER L'ÉCHEVEAU: LA PROCÉdURE EN SCHÉMA . . . . . . . 147

5.1 Clarifications terminologiques: naturalisation ordinaire, facilitée et simplifiée . . . . . 148

5.2 La procédure, un assemblage fédéral, cantonal et communal . . . . . . . . . . . . . . . . . . . . . 149

5.3 Les conditions de naturalisation selon les différentes sources . . . . . . . . . . . . . . . . . . . 154

5.3.1 La procédure d'acquisition de la nationalité dans la loi fédérale . . . . . . . . . . . . . . . . 154

5.3.2 Le processus de naturalisation ordinaire à Neuchâtel . . 156

5.3 .3 Coûts et durée de la procédure . . . . . . . . . . . . . 159

5.4 Schémas de visualisation des procédures ........... 161

5.4.1 Première phase: la demande d'autorisation fédérale ... 162

5.4.2 Seconde phase: procédure de demande de droit de cité communal et cantonal . . . . . . . . . . 165

5.4 .3 Les voies de recours . . . . . . . . . . . . . . . . 167

5.5 Mise à jour: la révision totale de la loi sur la nationalité. . 168

5.5 .1 Genèse ............................ 168

5.5.2 Des modifications de fond............... 170

5.5.3 Des changements de procédure............. 171

5.5.4 Compléments ........................... 174

5.6 Synthèse: une entreprise de clarification ......... 175 
PARTIE III. ANALYSE DES TRAJECTOIRES .

\section{FAIRE L'EXPÉRIENCE DES PROCÉDURES:}

TRAJECTOIRES DE VIE . . . . . . . . . . . . . . . . . . . . . . . . . . . 179

6.1 Penser et analyser les trajectoires de vie . . . . . . . . . 179

6.1.1 Théoriser le cours de la vie et l'imagination. . . . . . . . 180

6.1 .2 Analyser des trajectoires de vie . . . . . . . . . . 181

6.2 Un cas exemplaire: M. Charles.............. 182

6.2.1 Avant la demande: ruptures et transitions ......... 183

6.2.2 Entamer une démarche administrative:

la demande de naturalisation. . . . . . . . . . . . . 187

6.2.3 Etre confronté au travail de l'administration . . . . . . . 188

6.2 .4 Le sens personnel du refus . . . . . . . . . . . . . 192

6.2.5 Les démarches de naturalisation

dans la trajectoire biographique . . . . . . . . . . . . . 194

6.3 Lecture transversale des cas . . . . . . . . . . . . . . . . 195

6.3.1 Avant la demande . . . . . . . . . . . . . . . . . . . . . 196

6.3.2 Entamer une démarche administrative. . . . . . . . . . 197

6.3.3 Les personnes confrontées

au travail de l'administration. . . . . . . . . . . . . . . 200

6.3.4 Des raisons de refus et de leurs conséquences . . . . . . . 204

6.3.5 Les démarches de naturalisation

dans la trajectoire biographique . . . . . . . . . . . . . 209

6.4 Synthèse: la transformation des trajectoires de vie . . . . 213

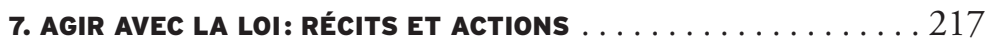

7.1 Conscience juridique et agentivité légale ......... 218

7.2 Construire et analyser les histoires légales . . . . . . . 220

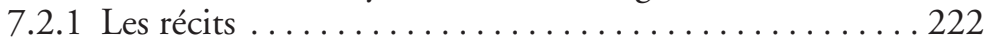

7.2 .2 Les dossiers . . . . . . . . . . . . . . . . . 223

7.3 Les cas de $\mathrm{M}^{\mathrm{me}}$ Line et MM. Charles, Kirin,

François et Johan . . . . . . . . . . . . . . . . . . . 224

7.3.1 Se positionner «avec le droit»:

le cas exemplaire de $\mathrm{M}^{\mathrm{me}}$ Line . . . . . . . . . . . . . . 225

7.3 .2 Engager un avocat. . . . . . . . . . . . . . . . . . 229

7.3.3 S'engager dans les échanges avec l'administration. . . . . 230

7.3 .4 Faire appel à la justice. . . . . . . . . . . . . . . 231

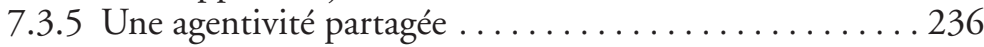

7.4 Différentes modalités d'agentivité.............. 238

7.4.1 Une agentivité suspendue: le cas de M. Charles ..... . 238 
7.4.2 Une agentivité planifiée: le cas de M. Kirin......... 245

7.4.3 Une agentivité déléguée et gagnante: le cas de M. François ... . . . . . . . . . . . . . . . 251

7.4.4 Une agentivité implicite et pleine: le cas de M. Johan . . 254

7.5 Synthèse: devenir actrice et acteur de son histoire légale. ................. 256

8. RENDRE DES DÉCISIONS: TRAJECTOIRE DES DOSSIERS . . . . . 263

8.1 Analyser la procédure de naturalisation . . . . . . . . . . 264

8.2 Une procédure de papier(s) . . . . . . . . . . . . 267

8.2 .1 Ce qu'est un dossier ....................... 268

8.2 .2 Traces, outils et résultats. ................ 271

8.3 Clore le dossier . . . . . . . . . . . . . . . . . . . 278

8.3.1 Du pouvoir de décider . . . . . . . . . . . . . . . . . 279

8.3.2 La procédure «recommandable» d'un canton «libéral». . 285

8.3.3 La force de l'influence et du consensus. . . . . . . . . . 290

8.4 Synthèse: la fragmentation du pouvoir décisionnel . . . 298

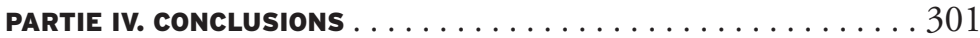

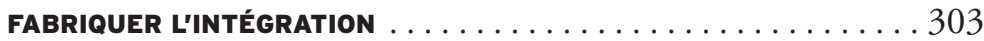

L'intégration, de la loi à la pratique . . . . . . . . . . . 303

Retour sur sept hypothèses.................... 306

D’une étude de cas à la recherche de propositions générales. . . 313 Ouvertures et implications pour la pratique............ 315

AUTEUR·E·S

BIBLIOGRAPHIE 
Impression

La Vallée - Aoste

Juin 2020 